\title{
Network Analysis of Substituted Bullvalenes
}

Oussama Yahiaoui, ${ }^{[a]}$ Lukáš F. Pašteka, ${ }^{*[b]}$ Christopher J. Blake, ${ }^{[c]}$ Christopher G. Newton ${ }^{[d]}$ and Thomas Fallon ${ }^{[a] *}$

${ }^{[a]}$ O. Yahiaoui, Dr. T. Fallon

Department of Chemistry, The University of Adelaide

Adelaide, SA 5005 (Australia)

Institute of Natural and Mathematical Sciences, Massey University1/5 University Ave, Albany, Auckland 0632, (New Zealand)E-mail: thomas.fallon@adelaide.edu.au

${ }^{[b]}$ Dept. of Physical and Theoretical Chemistry, Faculty of Natural Sciences, Comenius University, Ilkovičova 6, Bratislava, Slovakia

E-mail: lukas.f.pasteka@gmail.com

${ }^{[c]}$ Research School of Chemistry, Australian National University, Canberra, ACT 0200, Australia

${ }^{[d]}$ Department of Chemistry, The University of Adelaide Adelaide, SA 5005 (Australia)

bullvalene 4a: Single point DFT calculations at B3LYP-D3BJ/Def2-TZVPPD/CPCM (chloroform)

Vertex list:

isomer

energy

0000000012

25.6845

0000000021

41.2043

0000000102

13.3350

0000000120

23.1996

0000000201

25.6892

0000000210

20.9701

0000001002

17.2270

0000001020

9.8236

0000001200

29.8747

0000002001

30.5339

0000002010

7.2116

0000002100

28.7724

0000010020

0.0000

0000020010

0.0000

0000010200

2.5879

0000200010

2.5879

0000012000

7.7517

0002000010

7.7517

0000020100

6.3425

0000100020

6.3425

0000021000

10.0343 


$\begin{array}{lll}0001000020 & 10.0343 \\ 0000100200 & 7.7849 \\ 0000200100 & 7.7849 \\ 0000102000 & 12.0927 \\ 0002000100 & 12.0927 \\ 0000201000 & 11.2123 \\ 0001000200 & 11.2123 \\ 0001002000 & 31.8598 \\ 0002001000 & 31.8598\end{array}$

Edge list:

\begin{tabular}{|c|c|c|c|}
\hline isomer1 & $\operatorname{tran}$ & TS energy & \\
\hline 0000000012 & 0000002100 & 0000000012 & 76.9466 \\
\hline 0000000021 & 0000001200 & 0000000021 & 93.5749 \\
\hline 0000000102 & 0000002010 & 0000000102 & 58.4932 \\
\hline 0000000120 & 0000000210 & 0000000120 & 69.0757 \\
\hline 0000000201 & 0000001020 & 0000000201 & 70.3304 \\
\hline 0000001002 & 0000002001 & 0000001002 & 76.5738 \\
\hline 0000000012 & 0002001000 & 0000010002 & 72.9071 \\
\hline 0000000012 & 0001002000 & 0010000002 & 72.9071 \\
\hline 0000010020 & 0000201000 & 0000010020 & 52.4957 \\
\hline 0000020010 & 0001000200 & 0010000020 & 52.4957 \\
\hline 0000010200 & 0000021000 & 0000010200 & 53.1351 \\
\hline 0000200010 & 0001000020 & 0010000200 & 53.1351 \\
\hline 0000012000 & 0000001002 & 0000012000 & 59.4557 \\
\hline 0002000010 & 0000001002 & 0010002000 & 59.4557 \\
\hline 0000000021 & 0001002000 & 0000020001 & 90.1132 \\
\hline 0000000021 & 0002001000 & 0020000001 & 90.1132 \\
\hline 0000020010 & 0000102000 & 0000020010 & 58.0914 \\
\hline 0000010020 & 0002000100 & 0020000010 & 58.0914 \\
\hline 0000020100 & 0000012000 & 0000020100 & 58.3035 \\
\hline 0000100020 & 0002000010 & 0020000100 & 58.3035 \\
\hline 0000021000 & 0000002001 & 0000021000 & 77.1903 \\
\hline 0001000020 & 0000002001 & 0020001000 & 77.1903 \\
\hline 0000000102 & 0002000100 & 0000100002 & 66.3021 \\
\hline 0000000102 & 0000102000 & 0100000002 & 66.3021 \\
\hline 0000100020 & 0000200100 & 0000100020 & 59.8843 \\
\hline 0000020100 & 0000100200 & 0100000020 & 59.8843 \\
\hline 0000000120 & 0000002100 & 0000120000 & 78.6425 \\
\hline 0000000120 & 0000002100 & 0120000000 & 78.6425 \\
\hline 0000000201 & 0001000200 & 0000200001 & 81.2347 \\
\hline 0000000201 & 0000201000 & 0200000001 & 81.2347 \\
\hline 0000200010 & 0000100200 & 0000200010 & 62.7463 \\
\hline 0000010200 & 0000200100 & 0200000010 & 62.7463 \\
\hline 0000000210 & 0000001200 & 0000210000 & 75.4834 \\
\hline 0000000210 & 0000001200 & 0210000000 & 75.4834 \\
\hline
\end{tabular}




$\begin{array}{llll}0000001020 & 0000002010 & 0001020000 & 59.5028 \\ 0000001020 & 0000002010 & 1020000000 & 59.5028 \\ 0000010020 & 0002001000 & 0010020000 & 63.8429 \\ 0000020010 & 0001002000 & 0020010000 & 63.8429 \\ 0000010200 & 0000201000 & 0010200000 & 62.8388 \\ 0000200010 & 0001000200 & 0200010000 & 62.8388 \\ 0000020100 & 0000102000 & 0020100000 & 65.6455 \\ 0000100020 & 0002000100 & 0100020000 & 65.6455 \\ 0000021000 & 0000012000 & 0021000000 & 56.2353 \\ 0001000020 & 0002000010 & 1000020000 & 56.2353 \\ 0000100200 & 0000200100 & 0100200000 & 69.3756 \\ 0000200100 & 0000100200 & 0200100000 & 69.3756\end{array}$

bullvalene 4b: Single point DFT calculations at B3LYP-D3BJ/Def2-TZVPPD/CPCM (chloroform)

Vertex list:

\begin{tabular}{lrl} 
isomer & \multicolumn{3}{c}{ energy } \\
------ & \\
0000000012 & 33.8621 \\
0000000021 & 47.2986 \\
0000000102 & 21.6963 \\
0000000120 & 22.1571 \\
0000000201 & 23.6288 \\
0000000210 & 20.5209 \\
0000001002 & 24.5045 \\
0000001020 & 7.7198 \\
0000001200 & 35.1238 \\
0000002001 & 32.5877 \\
0000002010 & 11.0124 \\
0000002100 & 41.8554 \\
0000010020 & 0.7303 \\
0000020010 & 0.7303 \\
0000010200 & 0.0000 \\
0000200010 & 0.0000 \\
0000012000 & 8.5694 \\
0002000010 & 8.5694 \\
0000020100 & 3.7632 \\
0000100020 & 3.7632 \\
0000021000 & 11.6100 \\
0001000020 & 11.6100 \\
0000100200 & 4.0730 \\
0000200100 & 4.0730 \\
0000102000 & 13.8389 \\
0002000100 & 13.8389 \\
0000201000 & 7.9139 \\
0001000200 & 7.9139 \\
0001002000 & 32.0045 \\
0002001000 & 32.0045
\end{tabular}


Edge list:

\begin{tabular}{|c|c|c|c|}
\hline isomer1 & isomer2 & teTS energy & \\
\hline 0000000012 & 0000002100 & 0000000012 & 91.8950 \\
\hline 0000000021 & 0000001200 & 0000000021 & 99.6761 \\
\hline 0000000102 & 0000002010 & 0000000102 & 62.8273 \\
\hline 0000000120 & 0000000210 & 0000000120 & 68.7204 \\
\hline 0000000201 & 0000001020 & 0000000201 & 68.2853 \\
\hline 0000001002 & 0000002001 & 0000001002 & 81.1242 \\
\hline 0000000012 & 0002001000 & 0000010002 & 75.7142 \\
\hline 0000000012 & 0001002000 & 0010000002 & 75.7142 \\
\hline 0000010020 & 0000201000 & 0000010020 & 47.3447 \\
\hline 0000020010 & 0001000200 & 0010000020 & 47.3447 \\
\hline 0000010200 & 0000021000 & 0000010200 & 51.5274 \\
\hline 0000200010 & 0001000020 & 0010000200 & 51.5274 \\
\hline 0000012000 & 0000001002 & 0000012000 & 64.2012 \\
\hline 0002000010 & 0000001002 & 0010002000 & 64.2012 \\
\hline 0000000021 & 0001002000 & 0000020001 & 92.8995 \\
\hline 0000000021 & 0002001000 & 0020000001 & 92.8995 \\
\hline 0000020010 & 0000102000 & 0000020010 & 55.0252 \\
\hline 0000010020 & 0002000100 & 0020000010 & 55.0252 \\
\hline 0000020100 & 0000012000 & 0000020100 & 60.1252 \\
\hline 0000100020 & 0002000010 & 0020000100 & 60.1252 \\
\hline 0000021000 & 0000002001 & 0000021000 & 82.1941 \\
\hline 0001000020 & 0000002001 & 0020001000 & 82.1941 \\
\hline 0000000102 & 0002000100 & 0000100002 & 71.6968 \\
\hline 0000000102 & 0000102000 & 0100000002 & 71.6968 \\
\hline 0000100020 & 0000200100 & 0000100020 & 58.0255 \\
\hline 0000020100 & 0000100200 & 0100000020 & 58.0255 \\
\hline 0000000120 & 0000002100 & 0000120000 & 76.5620 \\
\hline 0000000120 & 0000002100 & 0120000000 & 76.5620 \\
\hline 0000000201 & 0001000200 & 0000200001 & 76.6737 \\
\hline 0000000201 & 0000201000 & 0200000001 & 76.6737 \\
\hline 0000200010 & 0000100200 & 0000200010 & 63.3289 \\
\hline 0000010200 & 0000200100 & 0200000010 & 63.3289 \\
\hline 0000000210 & 0000001200 & 0000210000 & 76.8175 \\
\hline 0000000210 & 0000001200 & 0210000000 & 76.8175 \\
\hline 0000001020 & 0000002010 & 0001020000 & 60.1983 \\
\hline 0000001020 & 0000002010 & 1020000000 & 60.1983 \\
\hline 0000010020 & 0002001000 & 0010020000 & 62.2013 \\
\hline 0000020010 & 0001002000 & 0020010000 & 62.2013 \\
\hline 0000010200 & 0000201000 & 0010200000 & 55.5454 \\
\hline 0000200010 & 0001000200 & 0200010000 & 55.5454 \\
\hline 0000012000 & 0000021000 & 0012000000 & 58.9385 \\
\hline 0002000010 & 0001000020 & 2000010000 & 58.9385 \\
\hline 0000020100 & 0000102000 & 0020100000 & 64.0643 \\
\hline
\end{tabular}




$\begin{array}{llll}0000100020 & 0002000100 & 0100020000 & 64.0643 \\ 0000100200 & 0000200100 & 0100200000 & 63.3743 \\ 0000200100 & 0000100200 & 0200100000 & 63.3743\end{array}$

bullvalene 4c: Single point DFT calculations at B3LYP-D3BJ/Def2-TZVPPD/CPCM (chloroform)

Vertex list:

\begin{tabular}{lrll} 
isomer & \multicolumn{2}{l}{ energy } \\
\hline------ & \\
0000000012 & 36.4942 \\
0000000021 & 45.9557 \\
0000000102 & 20.2104 \\
0000000120 & 20.7890 \\
0000000201 & 27.0965 \\
0000000210 & 18.4615 \\
0000001002 & 26.0483 \\
0000001020 & 10.8103 \\
0000001200 & 32.9086 \\
0000002001 & 34.1758 \\
0000002010 & 8.5155 \\
0000002100 & 37.2744 \\
0000010020 & 0.0000 \\
0000020010 & 0.0000 \\
0000010200 & 3.6966 \\
0000200010 & 3.6966 \\
0000012000 & 10.9808 \\
0002000010 & 10.9808 \\
0000020100 & 6.0057 \\
0000100020 & 6.0057 \\
0000021000 & 11.7586 \\
0001000020 & 11.7586 \\
0000100200 & 6.3717 \\
0000200100 & 6.3717 \\
0000102000 & 12.2034 \\
0002000100 & 12.2034 \\
0000201000 & 6.0112 \\
0001000200 & 6.0112 \\
0001002000 & 34.8525 \\
0002001000 & 34.8525
\end{tabular}

Edge list:

\begin{tabular}{lrrrrr} 
isomer1 & isomer2 & \multicolumn{3}{c}{ trans.stateTS energy } & \\
\hdashline---- & ---- & & \\
0000000012 & 0000002100 & 0000000012 & 88.4867 \\
0000000021 & 0000001200 & 0000000021 & 98.8263
\end{tabular}




$\begin{array}{llll}0000000102 & 0000002010 & 0000000102 & 63.1337 \\ 0000000120 & 0000000210 & 0000000120 & 67.1175 \\ 0000000201 & 0000001020 & 0000000201 & 71.7355 \\ 0000001002 & 0000002001 & 0000001002 & 85.2395 \\ 0000000012 & 0002001000 & 0000010002 & 78.8499 \\ 0000000012 & 0001002000 & 0010000002 & 78.8499 \\ 0000010020 & 0000201000 & 0000010020 & 45.2626 \\ 0000020010 & 0001000200 & 0010000020 & 45.2626 \\ 0000010200 & 0000021000 & 0000010200 & 55.0379 \\ 0000200010 & 0001000020 & 0010000200 & 55.0379 \\ 0000012000 & 0000001002 & 0000012000 & 68.3253 \\ 0002000010 & 0000001002 & 0010002000 & 68.3253 \\ 0000000021 & 0001002000 & 0000020001 & 89.7205 \\ 0000000021 & 0002001000 & 0020000001 & 89.7205 \\ 0000020010 & 0000102000 & 0000020010 & 54.4628 \\ 0000010020 & 0002000100 & 0020000010 & 54.4628 \\ 0000020100 & 0000012000 & 0000020100 & 60.9467 \\ 0000100020 & 0002000010 & 0020000100 & 60.9467 \\ 0000021000 & 0000002001 & 0000021000 & 80.3101 \\ 0001000020 & 0000002001 & 0020001000 & 80.3101 \\ 0000000102 & 0002000100 & 0000100002 & 71.7345 \\ 0000000102 & 0000102000 & 0100000002 & 71.7345 \\ 0000100020 & 0000200100 & 0000100020 & 61.4503 \\ 0000020100 & 0000100200 & 0100000020 & 61.4503 \\ 0000000120 & 0000002100 & 0000120000 & 77.2162 \\ 0000000120 & 0000002100 & 0120000000 & 77.2162 \\ 0000000201 & 0001000200 & 0000200001 & 82.0210 \\ 0000000201 & 0000201000 & 0200000001 & 82.0210 \\ 0000200010 & 0000100200 & 0000200010 & 64.3175 \\ 0000010200 & 0000200100 & 0200000010 & 64.3175 \\ 0000000210 & 0000001200 & 0000210000 & 74.4254 \\ 0000000210 & 0000001200 & 0210000000 & 74.4254 \\ 0000001020 & 0000002010 & 0001020000 & 61.8581 \\ 0000001020 & 0000002010 & 1020000000 & 61.8581 \\ 0000010020 & 0002001000 & 0010020000 & 83.3754 \\ 0000020010 & 0001002000 & 0020010000 & 83.3754 \\ 0000010200 & 0000201000 & 0010200000 & 58.4616 \\ 0000200010 & 0001000200 & 0200010000 & 58.4616 \\ 0000012000 & 0000021000 & 0012000000 & 62.9280 \\ 0002000010 & 0001000020 & 2000010000 & 62.9280 \\ 0000020100 & 0000102000 & 0020100000 & 66.5110 \\ 0000100020 & 0002000100 & 0100020000 & 66.5110 \\ 0000100200 & 0000200100 & 0100200000 & 63.3714 \\ 0000200100 & 0000100200 & 0200100000 & 63.3714\end{array}$

bullvalene 4d: Single point DFT calculations at B3LYP-D3BJ/Def2-TZVPPD/CPCM (chloroform)

Vertex list: 


\begin{tabular}{lrl} 
isomer & \multicolumn{2}{l}{ energy } \\
\hline------ & \\
0000000012 & 28.2006 \\
0000000021 & 47.0541 \\
0000000102 & 15.0848 \\
0000000120 & 22.3262 \\
0000000201 & 25.1414 \\
0000000210 & 21.0394 \\
0000001002 & 19.0497 \\
0000001020 & 7.9687 \\
0000001200 & 31.3582 \\
0000002001 & 30.3004 \\
0000002010 & 4.4828 \\
0000002100 & 30.8738 \\
0000010020 & 0.0000 \\
0000020010 & 0.0000 \\
0000010200 & 2.2508 \\
0000200010 & 2.2508 \\
0000012000 & 4.7010 \\
0002000010 & 4.7010 \\
0000020100 & 4.5880 \\
0000100020 & 4.5880 \\
0000021000 & 11.4129 \\
0001000020 & 11.4129 \\
0000100200 & 8.0341 \\
0000200100 & 8.0341 \\
0000102000 & 9.5926 \\
0002000100 & 9.5926 \\
0000201000 & 11.6625 \\
0001000200 & 11.6625 \\
0001002000 & 31.4534 \\
0002001000 & 31.4534
\end{tabular}

Edge list:

$\begin{array}{lrrrr}\text { isomer1 } & \text { isomer2 } & \text { trans.state TS energy } & \\ ------ & ---- & & \\ 0000000012 & 0000002100 & 0000000012 & 83.8281 \\ 0000000021 & 0000001200 & 0000000021 & 99.7322 \\ 0000000102 & 0000002010 & 0000000102 & 58.4873 \\ 0000000120 & 0000000210 & 0000000120 & 69.8914 \\ 0000000201 & 0000001020 & 0000000201 & 69.9296 \\ 0000001002 & 0000002001 & 0000001002 & 76.6219 \\ 0000000012 & 0002001000 & 0000010002 & 72.9460 \\ 0000000012 & 0001002000 & 0010000002 & 72.9460 \\ 0000010020 & 0000201000 & 0000010020 & 52.3858 \\ 0000020010 & 0001000200 & 0010000020 & 52.3858 \\ 0000010200 & 0000021000 & 0000010200 & 52.9301\end{array}$




$\begin{array}{llll}0000200010 & 0001000020 & 0010000200 & 52.9301 \\ 0000012000 & 0000001002 & 0000012000 & 58.2621 \\ 0002000010 & 0000001002 & 0010002000 & 58.2621 \\ 0000000021 & 0001002000 & 0000020001 & 93.9352 \\ 0000000021 & 0002001000 & 0020000001 & 93.9352 \\ 0000020010 & 0000102000 & 0000020010 & 55.5329 \\ 0000010020 & 0002000100 & 0020000010 & 55.5329 \\ 0000020100 & 0000012000 & 0000020100 & 58.6910 \\ 0000100020 & 0002000010 & 0020000100 & 58.6910 \\ 0000021000 & 0000002001 & 0000021000 & 77.1287 \\ 0001000020 & 0000002001 & 0020001000 & 77.1287 \\ 0000000102 & 0002000100 & 0000100002 & 66.0142 \\ 0000000102 & 0000102000 & 0100000002 & 66.0142 \\ 0000100020 & 0000200100 & 0000100020 & 59.6466 \\ 0000020100 & 0000100200 & 0100000020 & 59.6466 \\ 0000000120 & 0000002100 & 0000120000 & 75.3058 \\ 0000000120 & 0000002100 & 0120000000 & 75.3058 \\ 0000000201 & 0001000200 & 0000200001 & 83.0364 \\ 0000000201 & 0000201000 & 0200000001 & 83.0364 \\ 0000200010 & 0000100200 & 0000200010 & 70.0401 \\ 0000010200 & 0000200100 & 0200000010 & 70.0401 \\ 0000000210 & 0000001200 & 0000210000 & 75.7428 \\ 0000000210 & 0000001200 & 0210000000 & 75.7428 \\ 0000001020 & 0000002010 & 0001020000 & 59.5578 \\ 0000001020 & 0000002010 & 1020000000 & 59.5578 \\ 0000010020 & 0002001000 & 0010020000 & 64.8139 \\ 0000020010 & 0001002000 & 0020010000 & 64.8139 \\ 0000010200 & 0000201000 & 0010200000 & 64.9910 \\ 0000200010 & 0001000200 & 0200010000 & 64.9910 \\ 0000012000 & 0000021000 & 0012000000 & 59.4556 \\ 0002000010 & 0001000020 & 2000010000 & 59.4556 \\ 0000020100 & 0000102000 & 0020100000 & 66.4444 \\ 0000100020 & 0002000100 & 0100020000 & 66.4444 \\ 0000100200 & 0000200100 & 0100200000 & 67.8139 \\ 0000200100 & 0000100200 & 0200100000 & 67.8139\end{array}$

bullvalene 4e: Single point DFT calculations at B3LYP-D3BJ/Def2-TZVPPD/CPCM (chloroform)

Vertex list:

\begin{tabular}{lrl} 
isomer & \multicolumn{3}{c}{ energy } \\
------ & & \\
000000001 & 1 & 60.0678 \\
0000000101 & 24.2068 \\
0000000110 & 45.2357 \\
0000001001 & 28.7919 \\
0000001010 & 4.5939 \\
0000001100 & 52.8828 \\
0000010010 & & 0.0000
\end{tabular}




$\begin{array}{lll}0000010100 & 1.5936 \\ 0000100010 & 1.5936 \\ 0000011000 & 5.7629 \\ 0001000010 & 5.7629 \\ 0000100100 & 6.7769 \\ 0000101000 & 8.2250 \\ 0001000100 & 8.2250 \\ 0001001000 & 49.8972\end{array}$

Edge list:

\begin{tabular}{lllll} 
isomer1 & isomer2 & trans.state TS energy & \\
\hdashline 0000000011 & 0000001100 & 0000000011 & 117.0589 \\
0000000101 & 0000001010 & 0000000101 & 68.9895 \\
0000000110 & 0000000110 & 0000000110 & 92.7066 \\
0000001001 & 0000001001 & 0000001001 & 87.1172 \\
0000000011 & 0001001000 & 0000010001 & 101.7171 \\
0000000011 & 0001001000 & 0010000001 & 101.7171 \\
0000010010 & 0000101000 & 0000010010 & 48.9651 \\
0000010010 & 0001000100 & 0010000010 & 48.9651 \\
0000010100 & 0000011000 & 0000010100 & 52.4564 \\
0000100010 & 0001000010 & 0010000100 & 52.4564 \\
0000011000 & 0000001001 & 0000011000 & 70.2564 \\
0001000010 & 0000001001 & 0010001000 & 70.2564 \\
0000000101 & 0001000100 & 0000100001 & 76.2686 \\
0000000101 & 0000101000 & 0100000001 & 76.2686 \\
0000100010 & 0000100100 & 0000100010 & 59.1147 \\
0000010100 & 0000100100 & 0100000010 & 59.1147 \\
0000000110 & 0000001100 & 0000110000 & 100.0254 \\
0000000110 & 0000001100 & 0110000000 & 100.0254 \\
0000001010 & 0000001010 & 0001010000 & 47.8260 \\
0000001010 & 0000001010 & 1010000000 & 47.8260 \\
0000010010 & 0001001000 & 0010010000 & 69.7539 \\
0000010100 & 0000101000 & 0010100000 & 55.1350 \\
0000100010 & 0001000100 & 0100010000 & 55.1350 \\
0000011000 & 0000011000 & 0011000000 & 52.7181 \\
0001000010 & 0001000010 & 1000010000 & 52.7181 \\
0000100100 & 0000100100 & 0100100000 & 64.5322
\end{tabular}

bullvalene 4f: Single point DFT calculations at B3LYP-D3BJ/Def2-TZVPPD/CPCM (chloroform)

Vertex list:

isomer energy

$0000000122 \quad 43.3085$ 


\begin{tabular}{|c|c|}
\hline 0000000212 & 73.6274 \\
\hline 0000000221 & 66.7556 \\
\hline 0000001022 & 22.8657 \\
\hline 0000001202 & 45.8018 \\
\hline 0000001220 & 54.1410 \\
\hline 0000002012 & 37.5548 \\
\hline 0000002021 & 56.2428 \\
\hline 0000002102 & 45.3832 \\
\hline 0000002120 & 78.6944 \\
\hline 0000002201 & 35.2326 \\
\hline 0000002210 & 37.6545 \\
\hline 0000010022 & 34.5124 \\
\hline 0000020012 & 34.512 \\
\hline 0000010202 & 34.786 \\
\hline 0000200012 & 34.7860 \\
\hline 0000010220 & 19.8683 \\
\hline 0000220010 & 19.8683 \\
\hline 0000012002 & 37.606 \\
\hline 0002000012 & 37.606 \\
\hline 0000012020 & 9.1692 \\
\hline 0002020010 & 9.1692 \\
\hline 0000012200 & 12.0918 \\
\hline 0002200010 & 12.0918 \\
\hline 0000020021 & 74.3888 \\
\hline 0000020102 & 19.075 \\
\hline 0000100022 & $19.075 \varsigma$ \\
\hline 0000020120 & 24.4584 \\
\hline 0000120020 & 24.458 \\
\hline 0000020201 & 53.519 \\
\hline 0000200021 & 53.519 \\
\hline 0000020210 & 22.8240 \\
\hline 0000210020 & 22.824 \\
\hline 0000021002 & 22.807 \\
\hline 0001000022 & 22.807 \\
\hline 0000021020 & 10.6076 \\
\hline 0001020020 & 10.6076 \\
\hline 0000021200 & 45.2756 \\
\hline 0001200020 & 45.2756 \\
\hline 0000022001 & 56.306 \\
\hline 0002000021 & 56.306 \\
\hline 0000022010 & 8.7441 \\
\hline 0002010020 & 8.7441 \\
\hline 0000022100 & 34.739 \\
\hline 0002100020 & 34.739 \\
\hline 0000100202 & 21.627 \\
\hline 0000200102 & 21.627 \\
\hline 0000100220 & 28.425 \\
\hline 0000220100 & 28.425 \\
\hline 0000102002 & 24.215 \\
\hline 0002000102 & 24.215 \\
\hline
\end{tabular}




\begin{tabular}{|c|c|}
\hline 0000102020 & 13.5506 \\
\hline 0002020100 & 13.5506 \\
\hline 0000102200 & 19.9872 \\
\hline 0002200100 & 19.9872 \\
\hline 0000120200 & 28.8280 \\
\hline 0000200120 & 28.8280 \\
\hline 0000122000 & 32.4513 \\
\hline 0002000120 & 32.4513 \\
\hline 0000200201 & 31.9517 \\
\hline 0000200210 & 28.0000 \\
\hline 0000210200 & 28.0000 \\
\hline 0000201002 & 26.826 \\
\hline 0001000202 & 26.8266 \\
\hline 0000201020 & 16.096 \\
\hline 0001020200 & 16.096 \\
\hline 0000201200 & 39.4308 \\
\hline 0001200200 & 39.430 \\
\hline 0000202001 & 37.501 \\
\hline 0002000201 & 37.501 \\
\hline 0000202010 & 14.0135 \\
\hline 0002010200 & 14.013 \\
\hline 0000202100 & 37.567 \\
\hline 0002100200 & 37.567 \\
\hline 0000212000 & 32.3506 \\
\hline 0002000210 & 32.3506 \\
\hline 0000221000 & 36.0086 \\
\hline 0001000220 & 36.0086 \\
\hline 0001002002 & 46.062 \\
\hline 0002001002 & 46.062 \\
\hline 0001002020 & 35.6260 \\
\hline 0002021000 & 35.626 \\
\hline 0001002200 & 41.706 \\
\hline 0002201000 & 41.706 \\
\hline 0001022000 & 35.150 \\
\hline 0002001020 & 35.150 \\
\hline 0001202000 & 69.405 \\
\hline 0002001200 & 69.405 \\
\hline 0002002001 & 37.470 \\
\hline 0002002010 & 13.864 \\
\hline 0002012000 & 13.864 \\
\hline 0002002100 & 61.950 \\
\hline 0002102000 & 61.950 \\
\hline 0010020020 & 0.0000 \\
\hline 0010020200 & 7.2798 \\
\hline 0010200020 & 7.2798 \\
\hline 0010022000 & 9.2958 \\
\hline 0012000020 & 9.2958 \\
\hline 001020020 & 9.1909 \\
\hline 0010202000 & 14.653 \\
\hline 0012000200 & 14.653 \\
\hline
\end{tabular}




$\begin{array}{lll}0012002000 & 14.5396 \\ 0020020100 & 6.9725 \\ 0020021000 & 11.4813 \\ 0020100200 & 11.3940 \\ 0020200100 & 11.3940 \\ 0020102000 & 13.6649 \\ 0022000100 & 13.6649 \\ 0020201000 & 15.7792 \\ 0021000200 & 15.7792 \\ 0021002000 & 35.5837 \\ 0022001000 & 35.5837 \\ 0100200200 & 14.7232 \\ 0100202000 & 19.6982 \\ 0102000200 & 19.6982 \\ 0102002000 & 18.1368 \\ 0200201000 & 23.6191 \\ 0201002000 & 38.1670 \\ 0202001000 & 38.1670 \\ 1002002000 & 71.4734\end{array}$

Edge list:

\begin{tabular}{|c|c|c|c|}
\hline isomer1 & er2 & TS energy & \\
\hline 0000000122 & 0000002210 & 0000000122 & 92.7200 \\
\hline 0000000212 & 0000002120 & 0000000212 & 127.5455 \\
\hline 0000000221 & 0000001220 & 0000000221 & 126.8869 \\
\hline 0000001022 & 0000002201 & 0000001022 & 85.3500 \\
\hline 0000001202 & 0000002021 & 0000001202 & 110.1174 \\
\hline 0000002012 & 0000002102 & 0000002012 & 93.7109 \\
\hline 0000010022 & 0002201000 & 0000010022 & 86.6897 \\
\hline 0000020012 & 0001002200 & 0010000022 & 86.6897 \\
\hline 0000010202 & 0002021000 & 0000010202 & 77.3239 \\
\hline 0000200012 & 0001002020 & 0010000202 & 77.3239 \\
\hline 0000010220 & 0000221000 & 0000010220 & 70.4518 \\
\hline 0000220010 & 0001000220 & 0010000220 & 70.4518 \\
\hline 0000012002 & 0002001002 & 0000012002 & 84.5378 \\
\hline 0002000012 & 0001002002 & 0010002002 & 84.5378 \\
\hline 0000012020 & 0000201002 & 0000012020 & 62.2722 \\
\hline 0002020010 & 0001000202 & 0010002020 & 62.2722 \\
\hline 0000012200 & 0000021002 & 0000012200 & 67.9111 \\
\hline 0002200010 & 0001000022 & 0010002200 & 67.9111 \\
\hline 0000020012 & 0002102000 & 0000020012 & 104.8476 \\
\hline 0000010022 & 0002002100 & 0020000012 & 104.8476 \\
\hline 0000020021 & 0001202000 & 0000020021 & 141.0203 \\
\hline 0000020021 & 0002001200 & 0020000021 & 141.0203 \\
\hline 0000020102 & 0002012000 & 0000020102 & 65.2569 \\
\hline 0000100022 & 0002002010 & 0020000102 & 65.2569 \\
\hline
\end{tabular}




\begin{tabular}{|c|c|c|c|}
\hline 0000020120 & 0000212000 & 0000020120 & 85.3665 \\
\hline 0000120020 & 0002000210 & 0020000120 & 85.3665 \\
\hline 0000020201 & 0001022000 & 0000020201 & 91.4524 \\
\hline 0000200021 & 0002001020 & 0020000201 & 91.4524 \\
\hline 0000020210 & 0000122000 & 0000020210 & 76.8844 \\
\hline 0000210020 & 0002000120 & 0020000210 & 76.8844 \\
\hline 0000021002 & 0002002001 & 0000021002 & 84.0933 \\
\hline 0001000022 & 0002002001 & 0020001002 & 84.0933 \\
\hline 0000021020 & 0000202001 & 0000021020 & 81.0667 \\
\hline 0001020020 & 0002000201 & 0020001020 & 81.0667 \\
\hline 0000021200 & 0000022001 & 0000021200 & 110.4035 \\
\hline 0001200020 & 0002000021 & 0020001200 & 110.4035 \\
\hline 0000022001 & 0001002002 & 0000022001 & 105.1867 \\
\hline 0002000021 & 0002001002 & 0020002001 & 105.1867 \\
\hline 0000022010 & 0000102002 & 0000022010 & 66.6324 \\
\hline 0002010020 & 0002000102 & 0020002010 & 66.6324 \\
\hline 0000022100 & 0000012002 & 0000022100 & 95.7015 \\
\hline 0002100020 & 0002000012 & 0020002100 & 95.7015 \\
\hline 0000100022 & 0002200100 & 0000100022 & 75.6008 \\
\hline 0000020102 & 0000102200 & 0100000022 & 75.6008 \\
\hline 0000100202 & 0002020100 & 0000100202 & 70.5454 \\
\hline 0000200102 & 0000102020 & 0100000202 & 70.5454 \\
\hline 0000100220 & 0000220100 & 0000100220 & 83.2247 \\
\hline 0000220100 & 0000100220 & 0100000220 & 83.2247 \\
\hline 0000102002 & 0002000102 & 0000102002 & 77.2941 \\
\hline 0002000102 & 0000102002 & 0100002002 & 77.2941 \\
\hline 0000000122 & 0002002100 & 0000120002 & 98.8529 \\
\hline 0000000122 & 0002102000 & 0120000002 & 98.8529 \\
\hline 0000120020 & 0000202100 & 0000120020 & 81.1112 \\
\hline 0000020120 & 0002100200 & 0120000020 & 81.1112 \\
\hline 0000120200 & 0000022100 & 0000120200 & 79.6557 \\
\hline 0000200120 & 0002100020 & 0120000200 & 79.6557 \\
\hline 0000122000 & 0000002102 & 0000122000 & 86.7785 \\
\hline 0002000120 & 0000002102 & 0120002000 & 86.7785 \\
\hline 0000200012 & 0002100200 & 0000200012 & 101.6679 \\
\hline 0000010202 & 0000202100 & 0200000012 & 101.6679 \\
\hline 0000200021 & 0001200200 & 0000200021 & 116.6522 \\
\hline 0000020201 & 0000201200 & 0200000021 & 116.6522 \\
\hline 0000200102 & 0002010200 & 0000200102 & 75.7170 \\
\hline 0000100202 & 0000202010 & 0200000102 & 75.7170 \\
\hline 0000200120 & 0000210200 & 0000200120 & 86.8523 \\
\hline 0000120200 & 0000200210 & 0200000120 & 86.8523 \\
\hline 0000200201 & 0001020200 & 0000200201 & 87.7401 \\
\hline 0000200201 & 0000201020 & 0200000201 & 87.7401 \\
\hline 0000201002 & 0002000201 & 0000201002 & 94.1295 \\
\hline 0001000202 & 0000202001 & 0200001002 & 94.1295 \\
\hline 0000000212 & 0002001200 & 0000210002 & 129.5421 \\
\hline 0000000212 & 0001202000 & 0210000002 & 129.5421 \\
\hline 0000210020 & 0000201200 & 0000210020 & 83.0720 \\
\hline 0000020210 & 0001200200 & 0210000020 & 83.0720 \\
\hline
\end{tabular}




\begin{tabular}{|c|c|c|c|}
\hline 0000210200 & 0000021200 & 0000210200 & 82.6959 \\
\hline 0000200210 & 0001200020 & 0210000200 & 82.6959 \\
\hline 0000212000 & 0000001202 & 0000212000 & 87.9697 \\
\hline 0002000210 & 0000001202 & 0210002000 & 87.9697 \\
\hline 0000000221 & 0001002200 & 0000220001 & 111.5832 \\
\hline 0000000221 & 0002201000 & 0220000001 & 111.5832 \\
\hline 0000220010 & 0000102200 & 0000220010 & 73.0419 \\
\hline 0000010220 & 0002200100 & 0220000010 & 73.0419 \\
\hline 0000220100 & 0000012200 & 0000220100 & 73.8909 \\
\hline 0000100220 & 0002200010 & 0220000100 & 73.8909 \\
\hline 0000221000 & 0000002201 & 0000221000 & 91.4888 \\
\hline 0001000220 & 0000002201 & 0220001000 & 91.4888 \\
\hline 0000001022 & 0002002010 & 0001020002 & 66.6255 \\
\hline 0000001022 & 0002012000 & 1020000002 & 66.6255 \\
\hline 0001020020 & 0000202010 & 0001020020 & 63.8056 \\
\hline 0000021020 & 0002010200 & 1020000020 & 63.8056 \\
\hline 0001020200 & 0000022010 & 0001020200 & 63.5986 \\
\hline 0000201020 & 0002010020 & 1020000200 & 63.5986 \\
\hline 0001022000 & 0000002012 & 0001022000 & 83.8459 \\
\hline 0002001020 & 0000002012 & 1020002000 & 83.8459 \\
\hline 0000001220 & 0000002210 & 0001220000 & 92.3569 \\
\hline 0000001220 & 0000002210 & 1220000000 & 92.3569 \\
\hline 0000002021 & 0001002020 & 0002020001 & 102.3219 \\
\hline 0000002021 & 0002021000 & 2020000001 & 102.3219 \\
\hline 0002020010 & 0000102020 & 0002020010 & 72.6097 \\
\hline 0000012020 & 0002020100 & 2020000010 & 72.6097 \\
\hline 0000002120 & 0000002120 & 0002120000 & 121.9456 \\
\hline 0000002120 & 0000002120 & 2120000000 & 121.9456 \\
\hline 0000010022 & 1002002000 & 0010020002 & 91.0447 \\
\hline 0000020012 & 1002002000 & 0020010002 & 91.0447 \\
\hline 0010020020 & 0202001000 & 0010020020 & 69.9241 \\
\hline 0010020020 & 0201002000 & 0020010020 & 69.9241 \\
\hline 0010020200 & 0022001000 & 0010020200 & 69.0307 \\
\hline 0010200020 & 0021002000 & 0020010200 & 69.0307 \\
\hline 0010022000 & 0002001002 & 0010022000 & 77.1718 \\
\hline 0012000020 & 0001002002 & 0020012000 & 77.1718 \\
\hline 0000010202 & 0201002000 & 0010200002 & 88.5965 \\
\hline 0000200012 & 0202001000 & 0200010002 & 88.5965 \\
\hline 0010200020 & 0200201000 & 0010200020 & 71.2319 \\
\hline 0010020200 & 0200201000 & 0200010020 & 71.2319 \\
\hline 0010200200 & 0020201000 & 0010200200 & 69.7742 \\
\hline 0010200200 & 0021000200 & 0200010200 & 69.7742 \\
\hline 0010202000 & 0000201002 & 0010202000 & 75.0563 \\
\hline 0012000200 & 0001000202 & 0200012000 & 75.0563 \\
\hline 0000010220 & 0002201000 & 0010220000 & 74.3378 \\
\hline 0000220010 & 0001002200 & 0220010000 & 74.3378 \\
\hline 0000012002 & 0021002000 & 0012000002 & 83.0264 \\
\hline 0002000012 & 0022001000 & 2000010002 & 83.0264 \\
\hline 0012000020 & 0021000200 & 0012000020 & 63.4175 \\
\hline 0010022000 & 0020201000 & 2000010020 & 63.4175 \\
\hline
\end{tabular}




\begin{tabular}{|c|c|c|c|}
\hline 0012000200 & 0020021000 & 0012000200 & 63.8545 \\
\hline 0010202000 & 0020021000 & 2000010200 & 63.8545 \\
\hline 0012002000 & 0000021002 & 0012002000 & 67.0633 \\
\hline 0012002000 & 0001000022 & 2000012000 & 67.0633 \\
\hline 0000012020 & 0002021000 & 0012020000 & 75.0042 \\
\hline 0002020010 & 0001002020 & 2020010000 & 75.0042 \\
\hline 0000012200 & 0000221000 & 0012200000 & 73.4713 \\
\hline 0002200010 & 0001000220 & 2200010000 & 73.4713 \\
\hline 0000020021 & 1002002000 & 0020020001 & 123.0212 \\
\hline 0010020020 & 0102002000 & 0020020010 & 69.8918 \\
\hline 0020020100 & 0012002000 & 0020020100 & 64.8797 \\
\hline 0020021000 & 0002002001 & 0020021000 & 82.6254 \\
\hline 0000020102 & 0102002000 & 0020100002 & 73.7469 \\
\hline 0000100022 & 0102002000 & 0100020002 & 73.7469 \\
\hline 0020020100 & 0102000200 & 0020100020 & 70.4400 \\
\hline 0020020100 & 0100202000 & 0100020020 & 70.4400 \\
\hline 0020100200 & 0020102000 & 0020100200 & 71.0964 \\
\hline 0020200100 & 0022000100 & 0100020200 & 71.0964 \\
\hline 0020102000 & 0000102002 & 0020102000 & 76.5362 \\
\hline 0022000100 & 0002000102 & 0100022000 & 76.5362 \\
\hline 0000020120 & 0002102000 & 0020120000 & 91.7219 \\
\hline 0000120020 & 0002002100 & 0120020000 & 91.7219 \\
\hline 0000020201 & 0202001000 & 0020200001 & 108.9573 \\
\hline 0000200021 & 0201002000 & 0200020001 & 108.9573 \\
\hline 0010020200 & 0100202000 & 0020200010 & 71.1031 \\
\hline 0010200020 & 0102000200 & 0200020010 & 71.1031 \\
\hline 0020200100 & 0010202000 & 0020200100 & 75.5700 \\
\hline 0020100200 & 0012000200 & 0200020100 & 75.5700 \\
\hline 0020201000 & 0000202001 & 0020201000 & 91.3664 \\
\hline 0021000200 & 0002000201 & 0200021000 & 91.3664 \\
\hline 0000020210 & 0001202000 & 0020210000 & 97.9033 \\
\hline 0000210020 & 0002001200 & 0210020000 & 97.9033 \\
\hline 0000021020 & 0002012000 & 0021020000 & 65.0506 \\
\hline 0001020020 & 0002002010 & 1020020000 & 65.0506 \\
\hline 0000021200 & 0000212000 & 0021200000 & 87.1260 \\
\hline 0001200020 & 0002000210 & 1200020000 & 87.1260 \\
\hline 0000022001 & 0022001000 & 0022000001 & 104.4963 \\
\hline 0002000021 & 0021002000 & 2000020001 & 104.4963 \\
\hline 0010022000 & 0022000100 & 0022000010 & 65.9528 \\
\hline 0012000020 & 0020102000 & 2000020010 & 65.9528 \\
\hline 0000022010 & 0001022000 & 0022010000 & 75.1016 \\
\hline 0002010020 & 0002001020 & 2010020000 & 75.1016 \\
\hline 0000022100 & 0000122000 & 0022100000 & 86.3454 \\
\hline 0002100020 & 0002000120 & 2100020000 & 86.3454 \\
\hline 0000100202 & 0102000200 & 0100200002 & 82.3021 \\
\hline 0000200102 & 0100202000 & 0200100002 & 82.3021 \\
\hline 0020100200 & 0100200200 & 0100200020 & 72.3695 \\
\hline 0020200100 & 0100200200 & 0200100020 & 72.3695 \\
\hline 0000100220 & 0002200100 & 0100220000 & 79.1536 \\
\hline 0000220100 & 0000102200 & 0220100000 & 79.1536 \\
\hline
\end{tabular}




$\begin{array}{lllll}0000102020 & 0002020100 & 0102020000 & 80.6755 \\ 0002020100 & 0000102020 & 2020100000 & 80.6755 \\ 0000120200 & 0000202100 & 0120200000 & 99.1725 \\ 0000200120 & 0002100200 & 0200120000 & 99.1725 \\ 0000200201 & 0200201000 & 0200200001 & 92.1590 \\ 0010200200 & 0100200200 & 0200200010 & 74.0213 \\ 0000200210 & 0001200200 & 0200210000 & 87.2798 \\ 0000210200 & 0000201200 & 0210200000 & 87.2798 \\ 0000201020 & 0002010200 & 0201020000 & 74.9803 \\ 0001020200 & 0000202010 & 1020200000 & 74.9803\end{array}$

bullvalene $\mathbf{4 g}$ : Single point DFT calculations at B3LYP-D3BJ/Def2-TZVPPD/CPCM (chloroform)

Vertex list:

\begin{tabular}{lrr} 
isomer & energy \\
\hline------ & \\
0000000123 & 47.9124 \\
0000000132 & 44.4150 \\
0000000213 & 71.8428 \\
0000000231 & 62.7484 \\
0000000312 & 73.2954 \\
0000000321 & 63.0102 \\
0000001023 & 26.8865 \\
0000001032 & 17.7955 \\
0000001203 & 46.6278 \\
0000001230 & 53.2302 \\
0000001302 & 44.5175 \\
0000001320 & 50.4531 \\
0000002013 & 35.2971 \\
0000002031 & 51.4838 \\
0000002103 & 38.7819 \\
0000002130 & 77.4123 \\
0000002301 & 32.0332 \\
0000002310 & 38.8072 \\
0000003012 & 31.7299 \\
0000003021 & 52.9222 \\
0000003102 & 44.7012 \\
0000003120 & 73.8926 \\
0000003201 & 43.5427 \\
0000003210 & 43.3446 \\
0000010023 & 34.6327 \\
0000020013 & 34.6327 \\
0000010032 & 33.3580 \\
0000030012 & 33.3580 \\
0000010203 & 35.5400 \\
0000200013 & 35.5400 \\
0000010230 & 20.6963 \\
0000230010 & 20.6963
\end{tabular}




\begin{tabular}{|c|c|}
\hline 0000010302 & 26.1680 \\
\hline 0000300012 & 26.1680 \\
\hline 0000010320 & 16.7335 \\
\hline 0000320010 & 16.7335 \\
\hline 0000012003 & 33.9873 \\
\hline 0002000013 & 33.9873 \\
\hline 0000012030 & 8.4720 \\
\hline 0002030010 & 8.4720 \\
\hline 0000012300 & 6.1767 \\
\hline 0002300010 & 6.1767 \\
\hline 0000013002 & 34.5946 \\
\hline 0003000012 & 34.5946 \\
\hline 0000013020 & 6.7561 \\
\hline 0003020010 & 6.7561 \\
\hline 0000013200 & 17.4103 \\
\hline 0003200010 & 17.4103 \\
\hline 0000020031 & 69.0800 \\
\hline 0000030021 & 69.0800 \\
\hline 0000020103 & 17.0807 \\
\hline 0000100023 & 17.0807 \\
\hline 0000020130 & 19.320? \\
\hline 0000130020 & 19.3203 \\
\hline 0000020301 & 48.1705 \\
\hline 0000300021 & 48.170 \\
\hline 0000020310 & 17.877 \\
\hline 0000310020 & 17.8773 \\
\hline 0000021003 & 25.363 \\
\hline 0001000023 & 25.363 \\
\hline 0000021030 & 9.7413 \\
\hline 0001030020 & 9.7413 \\
\hline 0000021300 & 34.7917 \\
\hline 0001300020 & 34.791 \\
\hline 0000023001 & 53.6089 \\
\hline 0003000021 & 53.608 \\
\hline 0000023010 & 8.8067 \\
\hline 0003010020 & 8.8067 \\
\hline 0000023100 & 32.888 \\
\hline 0003100020 & 32.888 \\
\hline 0000030102 & 11.768 \\
\hline 0000100032 & 11.768 \\
\hline 0000030120 & 22.730 \\
\hline 0000120030 & 22.730 \\
\hline 0000030201 & $49.081 \mathrm{~s}$ \\
\hline 0000200031 & $49.081 \mathrm{c}$ \\
\hline 0000030210 & 18.356 \\
\hline 0000210030 & 18.356 \\
\hline 0000031002 & 18.315 \\
\hline 0001000032 & 18.315 \\
\hline 0000031020 & 8.7204 \\
\hline 0001020030 & 8.7204 \\
\hline
\end{tabular}




\begin{tabular}{|c|c|}
\hline 0000031200 & 38.0582 \\
\hline 0001200030 & 38.0582 \\
\hline 0000032001 & 51.1226 \\
\hline 0002000031 & 51.1226 \\
\hline 0000032010 & 8.1103 \\
\hline 0002010030 & 8.1103 \\
\hline 0000032100 & 29.1199 \\
\hline 0002100030 & 29.1199 \\
\hline 0000100203 & 24.1052 \\
\hline 0000200103 & 24.1052 \\
\hline 0000100230 & 20.7324 \\
\hline 0000230100 & 20.7324 \\
\hline 0000100302 & 17.0303 \\
\hline 0000300102 & 17.0303 \\
\hline 0000100320 & 20.8781 \\
\hline 0000320100 & 20.8781 \\
\hline 0000102003 & 23.0463 \\
\hline 0002000103 & 23.0463 \\
\hline 0000102030 & 7.1234 \\
\hline 0002030100 & 7.1234 \\
\hline 0000102300 & 11.5994 \\
\hline 0002300100 & 11.5994 \\
\hline 0000103002 & 23.9746 \\
\hline 0003000102 & 23.9746 \\
\hline 0000103020 & 17.8006 \\
\hline 0003020100 & 17.8006 \\
\hline 0000103200 & 20.7685 \\
\hline 0003200100 & 20.7685 \\
\hline 0000120300 & 17.6764 \\
\hline 0000300120 & 17.6764 \\
\hline 0000123000 & 28.4570 \\
\hline 0003000120 & 28.4570 \\
\hline 0000130200 & 26.8503 \\
\hline 0000200130 & 26.8503 \\
\hline 0000132000 & 32.4946 \\
\hline 0002000130 & 32.4946 \\
\hline 0000200301 & 26.7966 \\
\hline 0000300201 & 26.7966 \\
\hline 0000200310 & 22.9432 \\
\hline 0000310200 & 22.9432 \\
\hline 0000201003 & 29.1780 \\
\hline 0001000203 & 29.1780 \\
\hline 0000201030 & 13.1078 \\
\hline 0001030200 & 13.1078 \\
\hline 0000201300 & 34.8390 \\
\hline 0001300200 & 34.8390 \\
\hline 0000203001 & 32.5069 \\
\hline 0003000201 & 32.5069 \\
\hline 0000203010 & 9.1682 \\
\hline 0003010200 & 9.1682 \\
\hline
\end{tabular}




\begin{tabular}{|c|c|}
\hline 0000203100 & 33.9741 \\
\hline 0003100200 & 33.9741 \\
\hline 0000210300 & 23.3169 \\
\hline 0000300210 & 23.3169 \\
\hline 0000213000 & 30.5510 \\
\hline 0003000210 & 30.5510 \\
\hline 0000231000 & 30.9303 \\
\hline 0001000230 & 30.9303 \\
\hline 0000301002 & 19.1150 \\
\hline 0001000302 & 19.1150 \\
\hline 0000301020 & $0.00 \%$ \\
\hline 0001020300 & $0.00 \%$ \\
\hline 0000301200 & 32.8850 \\
\hline 0001200300 & 32.8850 \\
\hline 0000302001 & 30.6741 \\
\hline 0002000301 & 30.6741 \\
\hline 0000302010 & 5.3858 \\
\hline 0002010300 & 5.3858 \\
\hline 0000302100 & 36.0876 \\
\hline 0002100300 & 36.0876 \\
\hline 0000312000 & 24.3914 \\
\hline 0002000310 & 24.3914 \\
\hline 0000321000 & 21.8942 \\
\hline 0001000320 & 21.8942 \\
\hline 0001002003 & 46.4946 \\
\hline 0002001003 & 46.4946 \\
\hline 0001002030 & 36.1549 \\
\hline 0002031000 & 36.1549 \\
\hline 0001002300 & 40.9546 \\
\hline 0002301000 & 40.9546 \\
\hline 0001003002 & 46.1947 \\
\hline 0003001002 & 46.1947 \\
\hline 0001003020 & 35.6519 \\
\hline 0003021000 & 35.6519 \\
\hline 0001003200 & 42.1890 \\
\hline 0003201000 & 42.1890 \\
\hline 0001023000 & 35.4320 \\
\hline 0003001020 & 35.4320 \\
\hline 0001032000 & 32.7824 \\
\hline 0002001030 & 32.7824 \\
\hline 0001203000 & 65.8543 \\
\hline 0003001200 & 65.8543 \\
\hline 0001302000 & 61.7460 \\
\hline 0002001300 & 61.7460 \\
\hline 0002003001 & 38.0265 \\
\hline 0003002001 & 38.0265 \\
\hline 0002003010 & 8.9997 \\
\hline 0003012000 & 8.9997 \\
\hline 0002003100 & 58.4374 \\
\hline 0003102000 & 58.4374 \\
\hline
\end{tabular}




\begin{tabular}{|c|c|}
\hline 0002013000 & 14.2845 \\
\hline 0003002010 & 14.2845 \\
\hline 0002103000 & 35.3433 \\
\hline 0003002100 & 35.3433 \\
\hline 0010020030 & 0.5622 \\
\hline 0010030020 & 0.5622 \\
\hline 0010020300 & 0.0000 \\
\hline 0010300020 & 0.0000 \\
\hline 0010023000 & 7.9753 \\
\hline 0013000020 & 7.9753 \\
\hline 0010030200 & 3.0215 \\
\hline 0010200030 & 3.0215 \\
\hline 0010032000 & 6.3686 \\
\hline 0012000030 & 6.3686 \\
\hline 0010200300 & 6.4254 \\
\hline 0010300200 & 6.4254 \\
\hline 0010203000 & 9.9453 \\
\hline 0013000200 & 9.9453 \\
\hline 0010302000 & 9.3402 \\
\hline 0012000300 & 9.3402 \\
\hline 0012003000 & 16.2983 \\
\hline 0013002000 & 16.2983 \\
\hline 0020030100 & 10.6555 \\
\hline 0020100030 & 10.6555 \\
\hline 0020031000 & 14.7153 \\
\hline 0021000030 & 14.7153 \\
\hline 0020100300 & 9.5861 \\
\hline 0020300100 & 9.5861 \\
\hline 0020103000 & 12.2417 \\
\hline 0023000100 & 12.2417 \\
\hline 0020301000 & 12.6324 \\
\hline 0021000300 & 12.6324 \\
\hline 0021003000 & 35.2744 \\
\hline 0023001000 & 35.2744 \\
\hline 0030100200 & 5.8267 \\
\hline 0030200100 & 5.8267 \\
\hline 0030102000 & 7.5870 \\
\hline 0032000100 & 7.5870 \\
\hline 0030201000 & 13.0762 \\
\hline 0031000200 & 13.0762 \\
\hline 0031002000 & 33.1680 \\
\hline 0032001000 & 33.1680 \\
\hline 0100200300 & 11.8060 \\
\hline 0100300200 & 11.8060 \\
\hline 0100203000 & 14.5979 \\
\hline 0103000200 & 14.5979 \\
\hline 0100302000 & 13.9715 \\
\hline 0102000300 & 13.9715 \\
\hline 010200300 & 16.2230 \\
\hline 0103002000 & 16.2230 \\
\hline
\end{tabular}




$\begin{array}{lll}0200301000 & 13.0067 \\ 0201000300 & 13.0067 \\ 0201003000 & 37.4120 \\ 0203001000 & 37.4120 \\ 0301002000 & 34.2638 \\ 0302001000 & 34.2638 \\ 1002003000 & 62.8416 \\ 1003002000 & 62.8416\end{array}$

bullvalene $\mathbf{4 g}$ OTBS $\rightarrow$ OTMS analogue: Single point DFT calculations at B3LYP-D3BJ/Def2-TZVPPD/CPCM (chloroform)

Vertex list:

\begin{tabular}{lrr} 
isomer & energy \\
\hline------ & \\
0000000123 & 50.8022 \\
0000000132 & 46.3411 \\
0000000213 & 72.7490 \\
0000000231 & 86.0818 \\
0000000312 & 75.0163 \\
0000000321 & 65.8711 \\
0000001023 & 29.7111 \\
0000001032 & 19.6956 \\
0000001203 & 56.7601 \\
0000001230 & 55.7079 \\
0000001302 & 49.7540 \\
0000001320 & 52.6597 \\
0000002013 & 43.4306 \\
0000002031 & 54.2432 \\
0000002103 & 46.9102 \\
0000002130 & 80.3583 \\
0000002301 & 33.4278 \\
0000002310 & 39.7823 \\
0000003012 & 33.4557 \\
0000003021 & 53.8092 \\
0000003102 & 47.5766 \\
0000003120 & 76.3399 \\
0000003201 & 43.2845 \\
0000003210 & 46.6736 \\
0000010023 & 39.5880 \\
0000020013 & 39.5880 \\
0000010032 & 35.4855 \\
0000030012 & 35.4855 \\
0000010203 & 44.0291 \\
0000200013 & 44.0291 \\
0000010230 & 23.8821 \\
0000230010 & 23.8821 \\
0000010302 & 27.4949 \\
0000300012 & 27.4949 \\
0000010320 & 19.1710
\end{tabular}




\begin{tabular}{|c|c|}
\hline 0000320010 & 19.1710 \\
\hline 0000012003 & 41.8931 \\
\hline 0002000013 & 41.8931 \\
\hline 0000012030 & 8.2748 \\
\hline 0002030010 & 8.2748 \\
\hline 0000012300 & 7.8390 \\
\hline 0002300010 & 7.8390 \\
\hline 0000013002 & 35.7695 \\
\hline 0003000012 & 35.7695 \\
\hline 0000013020 & 8.5662 \\
\hline 0003020010 & 8.5662 \\
\hline 0000013200 & 20.1696 \\
\hline 0003200010 & 20.1696 \\
\hline 0000020031 & 70.9703 \\
\hline 0000030021 & 70.9703 \\
\hline 0000020103 & 25.8215 \\
\hline 0000100023 & 25.8215 \\
\hline 0000020130 & 26.422 \\
\hline 0000130020 & 26.4226 \\
\hline 0000020301 & 49.9413 \\
\hline 0000300021 & 49.9413 \\
\hline 0000020310 & 23.551 \\
\hline 0000310020 & 23.551 \\
\hline 0000021003 & 30.0567 \\
\hline 0001000023 & 30.0567 \\
\hline 0000021030 & 7.6572 \\
\hline 0001030020 & 7.6572 \\
\hline 0000021300 & 37.9889 \\
\hline 0001300020 & $37.988 \subseteq$ \\
\hline 0000023001 & 54.7386 \\
\hline 0003000021 & 54.7386 \\
\hline 0000023010 & 7.0870 \\
\hline 0003010020 & 7.0870 \\
\hline 0000023100 & 37.4275 \\
\hline 0003100020 & 37.427 \\
\hline 0000030102 & 15.013 \\
\hline 0000100032 & 15.013 \\
\hline 0000030120 & 19.527 \\
\hline 0000120030 & 19.527 \\
\hline 0000030201 & 50.9682 \\
\hline 0000200031 & 50.9682 \\
\hline 0000030210 & 19.6978 \\
\hline 0000210030 & 19.6978 \\
\hline 0000031002 & 21.643 \\
\hline 0001000032 & 21.643 \\
\hline 0000031020 & 8.3502 \\
\hline 0001020030 & 8.3502 \\
\hline 0000031200 & 43.163 \\
\hline 0001200030 & 43.163 \\
\hline 0000032001 & $56.817 !$ \\
\hline
\end{tabular}




\begin{tabular}{|c|c|}
\hline 0002000031 & 56.8175 \\
\hline 0000032010 & 9.5128 \\
\hline 0002010030 & 9.5128 \\
\hline 0000032100 & 32.8829 \\
\hline 0002100030 & 32.8829 \\
\hline 0000100203 & 22.7389 \\
\hline 0000200103 & 22.7389 \\
\hline 0000100230 & 25.8552 \\
\hline 0000230100 & 25.8552 \\
\hline 0000100302 & 19.4545 \\
\hline 0000300102 & 19.4545 \\
\hline 0000100320 & 23.7953 \\
\hline 0000320100 & 23.795 \\
\hline 0000102003 & 24.292 \\
\hline 0002000103 & 24.2924 \\
\hline 0000102030 & 12.865 \\
\hline 0002030100 & 12.865 \\
\hline 0000102300 & 14.9079 \\
\hline 0002300100 & 14.9079 \\
\hline 0000103002 & 23.8937 \\
\hline 0003000102 & 23.8937 \\
\hline 0000103020 & 20.3695 \\
\hline 0003020100 & 20.3695 \\
\hline 0000103200 & 22.3308 \\
\hline 0003200100 & 22.3308 \\
\hline 0000120300 & 24.0553 \\
\hline 0000300120 & 24.0553 \\
\hline 0000123000 & 31.109 \\
\hline 0003000120 & 31.1099 \\
\hline 0000130200 & 29.1011 \\
\hline 0000200130 & 29.101 \\
\hline 0000132000 & 32.8878 \\
\hline 0002000130 & $32.887 \varepsilon$ \\
\hline 0000200301 & 26.239 \\
\hline 0000300201 & 26.2394 \\
\hline 0000200310 & 26.3412 \\
\hline 0000310200 & 26.3412 \\
\hline 0000201003 & $31.286 s$ \\
\hline 0001000203 & $31.286 \subseteq$ \\
\hline 0000201030 & 11.8522 \\
\hline 0001030200 & 11.8522 \\
\hline 0000201300 & 37.8591 \\
\hline 0001300200 & 37.859 \\
\hline 0000203001 & 33.9083 \\
\hline 0003000201 & 33.908 \\
\hline 0000203010 & 8.3011 \\
\hline 0003010200 & 8.3011 \\
\hline 0000203100 & 37.8165 \\
\hline 0003100200 & 37.8165 \\
\hline 000021030 & 27.688 \\
\hline
\end{tabular}




\begin{tabular}{|c|c|}
\hline 0000300210 & 27.6887 \\
\hline 0000213000 & 29.3867 \\
\hline 0003000210 & 29.3867 \\
\hline 0000231000 & 33.9106 \\
\hline 0001000230 & 33.9106 \\
\hline 0000301002 & 22.9641 \\
\hline 0001000302 & 22.9641 \\
\hline 0000301020 & 9.7464 \\
\hline 0001020300 & 9.7464 \\
\hline 0000301200 & 37.3482 \\
\hline 0001200300 & 37.3482 \\
\hline 0000302001 & 35.9959 \\
\hline 0002000301 & 35.9959 \\
\hline 0000302010 & 10.7834 \\
\hline 0002010300 & 10.7834 \\
\hline 0000302100 & 38.1916 \\
\hline 0002100300 & 38.1916 \\
\hline 0000312000 & 31.7324 \\
\hline 0002000310 & 31.7324 \\
\hline 0000321000 & 25.2587 \\
\hline 0001000320 & 25.2587 \\
\hline 0001002003 & 51.0701 \\
\hline 0002001003 & 51.0701 \\
\hline 0001002030 & 37.9860 \\
\hline 0002031000 & 37.9860 \\
\hline 0001002300 & 43.5155 \\
\hline 0002301000 & 43.5155 \\
\hline 0001003002 & 48.6458 \\
\hline 0003001002 & 48.6458 \\
\hline 0001003020 & 37.6461 \\
\hline 0003021000 & 37.6461 \\
\hline 0001003200 & 47.4318 \\
\hline 0003201000 & 47.4318 \\
\hline 0001023000 & 38.3857 \\
\hline 0003001020 & 38.3857 \\
\hline 0001032000 & 33.9241 \\
\hline 0002001030 & 33.9241 \\
\hline 0001203000 & 71.8168 \\
\hline 0003001200 & 71.8168 \\
\hline 0001302000 & 72.6746 \\
\hline 0002001300 & 72.6746 \\
\hline 0002003001 & 42.1013 \\
\hline 0003002001 & 42.1013 \\
\hline 0002003010 & 13.3627 \\
\hline 0003012000 & 13.3627 \\
\hline 0002003100 & 37.6172 \\
\hline 0003102000 & 37.6172 \\
\hline 0002013000 & 15.8752 \\
\hline 0003002010 & 15.8752 \\
\hline 0002103000 & 43.4537 \\
\hline
\end{tabular}




\begin{tabular}{|c|c|}
\hline 0003002100 & 43.4537 \\
\hline 0010020030 & 0.0000 \\
\hline 0010030020 & 0.0000 \\
\hline 0010020300 & 0.7254 \\
\hline 0010300020 & 0.7254 \\
\hline 0010023000 & 8.7840 \\
\hline 0013000020 & 8.7840 \\
\hline 0010030200 & 3.7820 \\
\hline 0010200030 & 3.7820 \\
\hline 0010032000 & 9.5037 \\
\hline 0012000030 & 9.5037 \\
\hline 0010200300 & 5.5637 \\
\hline 0010300200 & 5.5637 \\
\hline 0010203000 & 11.0861 \\
\hline 0013000200 & 11.0861 \\
\hline 0010302000 & 15.6033 \\
\hline 0012000300 & 15.6033 \\
\hline 0012003000 & 16.7278 \\
\hline 0013002000 & 16.7278 \\
\hline 0020030100 & 6.7753 \\
\hline 0020100030 & 6.7753 \\
\hline 0020031000 & 10.7927 \\
\hline 0021000030 & 10.7927 \\
\hline 0020100300 & 10.9643 \\
\hline 0020300100 & 10.9643 \\
\hline 0020103000 & 16.1481 \\
\hline 0023000100 & 16.1481 \\
\hline 0020301000 & 13.3485 \\
\hline 0021000300 & 13.3485 \\
\hline 0021003000 & 37.5563 \\
\hline 0023001000 & 37.5563 \\
\hline 0030100200 & 9.5734 \\
\hline 0030200100 & 9.5734 \\
\hline 0030102000 & 11.3623 \\
\hline 0032000100 & 11.3623 \\
\hline 0030201000 & 13.9137 \\
\hline 0031000200 & 13.9137 \\
\hline 0031002000 & 37.0344 \\
\hline 0032001000 & 37.0344 \\
\hline 0100200300 & 10.8738 \\
\hline 0100300200 & 10.8738 \\
\hline 0100203000 & 15.2209 \\
\hline 0103000200 & 15.2209 \\
\hline 0100302000 & 19.7941 \\
\hline 0102000300 & 19.7941 \\
\hline 0102003000 & 20.2800 \\
\hline 0103002000 & 20.2800 \\
\hline 0200301000 & 18.4958 \\
\hline 0201000300 & 18.4958 \\
\hline 0201003000 & 40.4087 \\
\hline
\end{tabular}




$\begin{array}{ll}0203001000 & 40.4087 \\ 0301002000 & 38.7415 \\ 0302001000 & 38.7415 \\ 1002003000 & 63.7120 \\ 1003002000 & 63.7120\end{array}$

Edge list:

\begin{tabular}{|c|c|c|c|}
\hline isomer1 & er2 & TS energy & \\
\hline 0000000123 & 0000003210 & 0000000123 & 100.7054 \\
\hline 0000000132 & 0000002310 & 0000000132 & 95.0207 \\
\hline 0000000213 & 0000003120 & 0000000213 & 123.6519 \\
\hline 0000000231 & 0000001320 & 0000000231 & 117.4225 \\
\hline 0000000312 & 0000002130 & 0000000312 & 128.2439 \\
\hline 0000000321 & 0000001230 & 0000000321 & 62.4113 \\
\hline 0000001023 & 0000003201 & 0000001023 & 92.8436 \\
\hline 0000001032 & 0000002301 & 0000001032 & 83.3020 \\
\hline 0000001203 & 0000003021 & 0000001203 & 113.3823 \\
\hline 0000001302 & 0000002031 & 0000001302 & 109.1667 \\
\hline 0000002013 & 0000003102 & 0000002013 & 91.4077 \\
\hline 0000002103 & 0000003012 & 0000002103 & 93.1214 \\
\hline 0000010023 & 0003201000 & 0000010023 & 92.5073 \\
\hline 0000020013 & 0001003200 & 0010000023 & 92.5073 \\
\hline 0000010032 & 0002301000 & 0000010032 & 89.3193 \\
\hline 0000030012 & 0001002300 & 0010000032 & 89.3193 \\
\hline 0000010203 & 0003021000 & 0000010203 & 87.6616 \\
\hline 0000200013 & 0001003020 & 0010000203 & 87.6616 \\
\hline 0000010230 & 0000321000 & 0000010230 & 69.5745 \\
\hline 0000230010 & 0001000320 & 0010000230 & 69.5745 \\
\hline 0000010302 & 0002031000 & 0000010302 & 77.9276 \\
\hline 0000300012 & 0001002030 & 0010000302 & 77.9276 \\
\hline 0000010320 & 0000231000 & 0000010320 & 69.2313 \\
\hline 0000320010 & 0001000230 & 0010000320 & 69.2313 \\
\hline 0000012003 & 0003001002 & 0000012003 & 91.6505 \\
\hline 0002000013 & 0001003002 & 0010002003 & 91.6505 \\
\hline 0000012030 & 0000301002 & 0000012030 & 58.7182 \\
\hline 0002030010 & 0001000302 & 0010002030 & 58.7182 \\
\hline 0000012300 & 0000031002 & 0000012300 & 68.1862 \\
\hline 0002300010 & 0001000032 & 0010002300 & 68.1862 \\
\hline 0000013002 & 0002001003 & 0000013002 & 88.2067 \\
\hline 0003000012 & 0001002003 & 0010003002 & 88.2067 \\
\hline 0000013020 & 0000201003 & 0000013020 & 67.7816 \\
\hline 0003020010 & 0001000203 & 0010003020 & 67.7816 \\
\hline 0000013200 & 0000021003 & 0000013200 & 76.2323 \\
\hline 0003200010 & 0001000023 & 0010003200 & 76.2323 \\
\hline 0000020013 & 0003102000 & 0000020013 & 103.1411 \\
\hline 0000010023 & 0002003100 & 0020000013 & 103.1411 \\
\hline
\end{tabular}




\begin{tabular}{|c|c|c|c|}
\hline 0000020031 & 0001302000 & 0000020031 & 133.3256 \\
\hline 0000030021 & 0002001300 & 0020000031 & 133.3256 \\
\hline 0000020103 & 0003012000 & 0000020103 & 75.8643 \\
\hline 0000100023 & 0002003010 & 0020000103 & 75.8643 \\
\hline 0000020130 & 0000312000 & 0000020130 & 36.8482 \\
\hline 0000130020 & 0002000310 & 0020000130 & 36.8482 \\
\hline 0000020301 & 0001032000 & 0000020301 & 95.4719 \\
\hline 0000300021 & 0002001030 & 0020000301 & 95.4719 \\
\hline 0000020310 & 0000132000 & 0000020310 & 79.0522 \\
\hline 0000310020 & 0002000130 & 0020000310 & 79.0522 \\
\hline 0000021003 & 0003002001 & 0000021003 & 91.7779 \\
\hline 0001000023 & 0002003001 & 0020001003 & 91.7779 \\
\hline 0000021030 & 0000302001 & 0000021030 & 78.7784 \\
\hline 0001030020 & 0002000301 & 0020001030 & 78.7784 \\
\hline 0000021300 & 0000032001 & 0000021300 & 108.3020 \\
\hline 0001300020 & 0002000031 & 0020001300 & 108.3020 \\
\hline 0000023001 & 0001002003 & 0000023001 & 109.0014 \\
\hline 0003000021 & 0002001003 & 0020003001 & 109.0014 \\
\hline 0000023010 & 0000102003 & 0000023010 & 68.9386 \\
\hline 0003010020 & 0002000103 & 0020003010 & 68.9386 \\
\hline 0000023100 & 0000012003 & 0000023100 & 91.8520 \\
\hline 0003100020 & 0002000013 & 0020003100 & 91.8520 \\
\hline 0000030012 & 0002103000 & 0000030012 & 89.0789 \\
\hline 0000010032 & 0003002100 & 0030000012 & 89.0789 \\
\hline 0000030021 & 0001203000 & 0000030021 & 139.3215 \\
\hline 0000020031 & 0003001200 & 0030000021 & 139.3215 \\
\hline 0000030102 & 0002013000 & 0000030102 & 64.9905 \\
\hline 0000100032 & 0003002010 & 0030000102 & 64.9905 \\
\hline 0000030120 & 0000213000 & 0000030120 & 79.9028 \\
\hline 0000120030 & 0003000210 & 0030000120 & 79.9028 \\
\hline 0000030201 & 0001023000 & 0000030201 & 102.2778 \\
\hline 0000200031 & 0003001020 & 0030000201 & 102.2778 \\
\hline 0000030210 & 0000123000 & 0000030210 & 72.9854 \\
\hline 0000210030 & 0003000120 & 0030000210 & 72.9854 \\
\hline 0000031002 & 0002003001 & 0000031002 & 85.5239 \\
\hline 0001000032 & 0003002001 & 0030001002 & 85.5239 \\
\hline 0000031020 & 0000203001 & 0000031020 & 85.3015 \\
\hline 0001020030 & 0003000201 & 0030001020 & 85.3015 \\
\hline 0000031200 & 0000023001 & 0000031200 & 105.5087 \\
\hline 0001200030 & 0003000021 & 0030001200 & 105.5087 \\
\hline 0000032001 & 0001003002 & 0000032001 & 103.9886 \\
\hline 0002000031 & 0003001002 & 0030002001 & 103.9886 \\
\hline 0000032010 & 0000103002 & 0000032010 & 65.4332 \\
\hline 0002010030 & 0003000102 & 0030002010 & 65.4332 \\
\hline 0000032100 & 0000013002 & 0000032100 & 88.8301 \\
\hline 0002100030 & 0003000012 & 0030002100 & 88.8301 \\
\hline 0000100023 & 0003200100 & 0000100023 & 77.2211 \\
\hline 0000020103 & 0000103200 & 0100000023 & 77.2211 \\
\hline 0000100032 & 0002300100 & 0000100032 & 73.8677 \\
\hline 0000030102 & 0000102300 & 0100000032 & 73.8677 \\
\hline
\end{tabular}




\begin{tabular}{|c|c|c|c|}
\hline 0000100203 & 0003020100 & 0000100203 & 69.2174 \\
\hline 0000200103 & 0000103020 & 0100000203 & 69.2174 \\
\hline 0000100230 & 0000320100 & 0000100230 & 75.6760 \\
\hline 0000230100 & 0000100320 & 0100000230 & 75.6760 \\
\hline 0000100302 & 0002030100 & 0000100302 & 68.9996 \\
\hline 0000300102 & 0000102030 & 0100000302 & 68.9996 \\
\hline 0000102003 & 0003000102 & 0000102003 & 75.8630 \\
\hline 0002000103 & 0000103002 & 0100002003 & 75.8630 \\
\hline 0000000123 & 0003002100 & 0000120003 & 99.4828 \\
\hline 0000000123 & 0002103000 & 0120000003 & 99.4828 \\
\hline 0000120030 & 0000302100 & 0000120030 & 76.1557 \\
\hline 0000030120 & 0002100300 & 0120000030 & 76.1557 \\
\hline 0000120300 & 0000032100 & 0000120300 & 29.0706 \\
\hline 0000300120 & 0002100030 & 0120000300 & 29.0706 \\
\hline 0000123000 & 0000002103 & 0000123000 & 85.4950 \\
\hline 0003000120 & 0000002103 & 0120003000 & 85.4950 \\
\hline 0000000132 & 0002003100 & 0000130002 & 49.6918 \\
\hline 0000000132 & 0003102000 & 0130000002 & 49.6918 \\
\hline 0000130020 & 0000203100 & 0000130020 & 87.0211 \\
\hline 0000020130 & 0003100200 & 0130000020 & 87.0211 \\
\hline 0000130200 & 0000023100 & 0000130200 & 82.6799 \\
\hline 0000200130 & 0003100020 & 0130000200 & 82.6799 \\
\hline 0000132000 & 0000003102 & 0000132000 & 90.4653 \\
\hline 0002000130 & 0000003102 & 0130002000 & 90.4653 \\
\hline 0000200013 & 0003100200 & 0000200013 & 100.0618 \\
\hline 0000010203 & 0000203100 & 0200000013 & 100.0618 \\
\hline 0000200031 & 0001300200 & 0000200031 & 115.1023 \\
\hline 0000030201 & 0000201300 & 0200000031 & 115.1023 \\
\hline 0000200103 & 0003010200 & 0000200103 & 76.2001 \\
\hline 0000100203 & 0000203010 & 0200000103 & 76.2001 \\
\hline 0000200130 & 0000310200 & 0000200130 & 84.7595 \\
\hline 0000130200 & 0000200310 & 0200000130 & 84.7595 \\
\hline 0000200301 & 0001030200 & 0000200301 & 83.0617 \\
\hline 0000300201 & 0000201030 & 0200000301 & 83.0617 \\
\hline 0000201003 & 0003000201 & 0000201003 & 94.7797 \\
\hline 0001000203 & 0000203001 & 0200001003 & 94.7797 \\
\hline 0000000213 & 0003001200 & 0000210003 & 138.4698 \\
\hline 0000000213 & 0001203000 & 0210000003 & 138.4698 \\
\hline 0000210030 & 0000301200 & 0000210030 & 75.0555 \\
\hline 0000030210 & 0001200300 & 0210000030 & 75.0555 \\
\hline 0000210300 & 0000031200 & 0000210300 & 79.6762 \\
\hline 0000300210 & 0001200030 & 0210000300 & 79.6762 \\
\hline 0000213000 & 0000001203 & 0000213000 & 92.4546 \\
\hline 0003000210 & 0000001203 & 0210003000 & 92.4546 \\
\hline 0000000231 & 0001003200 & 0000230001 & 113.1145 \\
\hline 0000000231 & 0003201000 & 0230000001 & 113.1145 \\
\hline 0000230010 & 0000103200 & 0000230010 & 77.1860 \\
\hline 0000010230 & 0003200100 & 0230000010 & 77.1860 \\
\hline 0000230100 & 0000013200 & 0000230100 & 74.3552 \\
\hline 0000100230 & 0003200010 & 0230000100 & 74.3552 \\
\hline
\end{tabular}




\begin{tabular}{|c|c|c|c|}
\hline 0000231000 & 0000003201 & 0000231000 & 94.6126 \\
\hline 0001000230 & 0000003201 & 0230001000 & 94.6126 \\
\hline 0000300012 & 0002100300 & 0000300012 & 98.0654 \\
\hline 0000010302 & 0000302100 & 0300000012 & 98.0654 \\
\hline 0000300021 & 0001200300 & 0000300021 & 111.2067 \\
\hline 0000020301 & 0000301200 & 0300000021 & 111.2067 \\
\hline 0000300102 & 0002010300 & 0000300102 & 71.9358 \\
\hline 0000100302 & 0000302010 & 0300000102 & 71.9358 \\
\hline 0000300120 & 0000210300 & 0000300120 & 80.8691 \\
\hline 0000120300 & 0000300210 & 0300000120 & 80.8691 \\
\hline 0000300201 & 0001020300 & 0000300201 & 80.6404 \\
\hline 0000200301 & 0000301020 & 0300000201 & 80.6404 \\
\hline 0000301002 & 0002000301 & 0000301002 & 90.9384 \\
\hline 0001000302 & 0000302001 & 0300001002 & 90.9384 \\
\hline 0000000312 & 0002001300 & 0000310002 & 122.9724 \\
\hline 0000000312 & 0001302000 & 0310000002 & 122.9724 \\
\hline 0000310020 & 0000201300 & 0000310020 & 82.1052 \\
\hline 0000020310 & 0001300200 & 0310000020 & 82.1052 \\
\hline 0000310200 & 0000021300 & 0000310200 & 81.3029 \\
\hline 0000200310 & 0001300020 & 0310000200 & 81.3029 \\
\hline 0000312000 & 0000001302 & 0000312000 & 89.9074 \\
\hline 0002000310 & 0000001302 & 0310002000 & 89.9074 \\
\hline 0000000321 & 0001002300 & 0000320001 & 112.0289 \\
\hline 0000000321 & 0002301000 & 0320000001 & 112.0289 \\
\hline 0000320010 & 0000102300 & 0000320010 & 70.1357 \\
\hline 0000010320 & 0002300100 & 0320000010 & 70.1357 \\
\hline 0000320100 & 0000012300 & 0000320100 & 70.6230 \\
\hline 0000100320 & 0002300010 & 0320000100 & 70.6230 \\
\hline 0000321000 & 0000002301 & 0000321000 & 88.0388 \\
\hline 0001000320 & 0000002301 & 0320001000 & 88.0388 \\
\hline 0000001023 & 0003002010 & 0001020003 & 72.7103 \\
\hline 0000001023 & 0002013000 & 1020000003 & 72.7103 \\
\hline 0001020030 & 0000302010 & 0001020030 & 57.2441 \\
\hline 0000031020 & 0002010300 & 1020000030 & 57.2441 \\
\hline 0001020300 & 0000032010 & 0001020300 & 58.5265 \\
\hline 0000301020 & 0002010030 & 1020000300 & 58.5265 \\
\hline 0001023000 & 0000002013 & 0001023000 & 86.1395 \\
\hline 0003001020 & 0000002013 & 1020003000 & 86.1395 \\
\hline 0000001032 & 0002003010 & 0001030002 & 62.9828 \\
\hline 0000001032 & 0003012000 & 1030000002 & 62.9828 \\
\hline 0001030020 & 0000203010 & 0001030020 & 65.2313 \\
\hline 0000021030 & 0003010200 & 1030000020 & 65.2313 \\
\hline 0001030200 & 0000023010 & 0001030200 & 55.7427 \\
\hline 0000201030 & 0003010020 & 1030000200 & 55.7427 \\
\hline 0001032000 & 0000003012 & 0001032000 & 78.5892 \\
\hline 0002001030 & 0000003012 & 1030002000 & 78.5892 \\
\hline 0000001230 & 0000003210 & 0001230000 & 118.6920 \\
\hline 0000001230 & 0000003210 & 1230000000 & 118.6920 \\
\hline 0000001320 & 0000002310 & 0001320000 & 94.5449 \\
\hline 0000001320 & 0000002310 & 1320000000 & 94.5449 \\
\hline
\end{tabular}




\begin{tabular}{|c|c|c|c|}
\hline 0000002031 & 0001003020 & 0002030001 & 102.9558 \\
\hline 0000002031 & 0003021000 & 2030000001 & 102.9558 \\
\hline 0002030010 & 0000103020 & 0002030010 & 64.1803 \\
\hline 0000012030 & 0003020100 & 2030000010 & 64.1803 \\
\hline 0002030100 & 0000013020 & 0002030100 & 59.4176 \\
\hline 0000102030 & 0003020010 & 2030000100 & 59.4176 \\
\hline 0002031000 & 0000003021 & 0002031000 & 101.2379 \\
\hline 0001002030 & 0000003021 & 2030001000 & 101.2379 \\
\hline 0000002130 & 0000003120 & 0002130000 & 130.3370 \\
\hline 0000002130 & 0000003120 & 2130000000 & 130.3370 \\
\hline 0000010023 & 1003002000 & 0010020003 & 95.6128 \\
\hline 0000020013 & 1002003000 & 0020010003 & 95.6128 \\
\hline 0010020030 & 0302001000 & 0010020030 & 67.1392 \\
\hline 0010030020 & 0301002000 & 0020010030 & 67.1392 \\
\hline 0010020300 & 0032001000 & 0010020300 & 70.0412 \\
\hline 0010300020 & 0031002000 & 0020010300 & 70.0412 \\
\hline 0010023000 & 0002001003 & 0010023000 & 81.4986 \\
\hline 0013000020 & 0001002003 & 0020013000 & 81.4986 \\
\hline 0000010032 & 1002003000 & 0010030002 & 94.7409 \\
\hline 0000030012 & 1003002000 & 0030010002 & 94.7409 \\
\hline 0010030020 & 0203001000 & 0010030020 & 70.6248 \\
\hline 0010020030 & 0201003000 & 0030010020 & 70.6248 \\
\hline 0010030200 & 0023001000 & 0010030200 & 64.9659 \\
\hline 0010200030 & 0021003000 & 0030010200 & 64.9659 \\
\hline 0010032000 & 0003001002 & 0010032000 & 73.8951 \\
\hline 0012000030 & 0001003002 & 0030012000 & 73.8951 \\
\hline 0000010203 & 0201003000 & 0010200003 & 89.8023 \\
\hline 0000200013 & 0203001000 & 0200010003 & 89.8023 \\
\hline 0010200030 & 0201000300 & 0010200030 & 64.2856 \\
\hline 0010030200 & 0200301000 & 0200010030 & 64.2856 \\
\hline 0010200300 & 0030201000 & 0010200300 & 67.7452 \\
\hline 0010300200 & 0031000200 & 0200010300 & 67.7452 \\
\hline 0010203000 & 0000201003 & 0010203000 & 78.0901 \\
\hline 0013000200 & 0001000203 & 0200013000 & 78.0901 \\
\hline 0000010230 & 0003201000 & 0010230000 & 77.7094 \\
\hline 0000230010 & 0001003200 & 0230010000 & 77.7094 \\
\hline 0000010302 & 0301002000 & 0010300002 & 89.1762 \\
\hline 0000300012 & 0302001000 & 0300010002 & 89.1762 \\
\hline 0010300020 & 0200301000 & 0010300020 & 68.5313 \\
\hline 0010020300 & 0201000300 & 0300010020 & 68.5313 \\
\hline 0010300200 & 0020301000 & 0010300200 & 66.7557 \\
\hline 0010200300 & 0021000300 & 0300010200 & 66.7557 \\
\hline 0010302000 & 0000301002 & 0010302000 & 75.6726 \\
\hline 0012000300 & 0001000302 & 0300012000 & 75.6726 \\
\hline 0000010320 & 0002301000 & 0010320000 & 77.2237 \\
\hline 0000320010 & 0001002300 & 0320010000 & 77.2237 \\
\hline 0000012003 & 0021003000 & 0012000003 & 85.1163 \\
\hline 0002000013 & 0023001000 & 2000010003 & 85.1163 \\
\hline 0012000030 & 0021000300 & 0012000030 & 58.7434 \\
\hline 0010032000 & 0020301000 & 2000010030 & 58.7434 \\
\hline
\end{tabular}




\begin{tabular}{|c|c|c|c|}
\hline 0012000300 & 0021000030 & 0012000300 & 64.8557 \\
\hline 0010302000 & 0020031000 & 2000010300 & 64.8557 \\
\hline 0012003000 & 0000021003 & 0012003000 & 72.6566 \\
\hline 0013002000 & 0001000023 & 2000013000 & 72.6566 \\
\hline 0000012030 & 0003021000 & 0012030000 & 70.9745 \\
\hline 0002030010 & 0001003020 & 2030010000 & 70.9745 \\
\hline 0000012300 & 0000321000 & 0012300000 & 74.4373 \\
\hline 0002300010 & 0001000320 & 2300010000 & 74.4373 \\
\hline 0000013002 & 0031002000 & 0013000002 & 77.7585 \\
\hline 0003000012 & 0032001000 & 3000010002 & 77.7585 \\
\hline 0013000020 & 0031000200 & 0013000020 & 60.9751 \\
\hline 0010023000 & 0030201000 & 3000010020 & 60.9751 \\
\hline 0013000200 & 0020031000 & 0013000200 & 69.6734 \\
\hline 0010203000 & 0021000030 & 3000010200 & 69.6734 \\
\hline 0013002000 & 0000031002 & 0013002000 & 67.4530 \\
\hline 0012003000 & 0001000032 & 3000012000 & 67.4530 \\
\hline 0000013020 & 0002031000 & 0013020000 & 71.5026 \\
\hline 0003020010 & 0001002030 & 3020010000 & 71.5026 \\
\hline 0000013200 & 0000231000 & 0013200000 & 76.6650 \\
\hline 0003200010 & 0001000230 & 3200010000 & 76.6650 \\
\hline 0000020031 & 1003002000 & 0020030001 & 120.3281 \\
\hline 0000030021 & 1002003000 & 0030020001 & 120.3281 \\
\hline 0010020030 & 0103002000 & 0020030010 & 67.2407 \\
\hline 0010030020 & 0102003000 & 0030020010 & 67.2407 \\
\hline 0020030100 & 0013002000 & 0020030100 & 69.0948 \\
\hline 0020100030 & 0012003000 & 0030020100 & 69.0948 \\
\hline 0020031000 & 0003002001 & 0020031000 & 85.2329 \\
\hline 0021000030 & 0002003001 & 0030021000 & 85.2329 \\
\hline 0000020103 & 0102003000 & 0020100003 & 86.1310 \\
\hline 0000100023 & 0103002000 & 0100020003 & 86.1310 \\
\hline 0020100030 & 0102000300 & 0020100030 & 86.8101 \\
\hline 0020030100 & 0100302000 & 0100020030 & 86.8101 \\
\hline 0020100300 & 0030102000 & 0020100300 & 69.0961 \\
\hline 0020300100 & 0032000100 & 0100020300 & 69.0961 \\
\hline 0020103000 & 0000102003 & 0020103000 & 75.4438 \\
\hline 0023000100 & 0002000103 & 0100023000 & 75.4438 \\
\hline 0000020130 & 0003102000 & 0020130000 & 84.3267 \\
\hline 0000130020 & 0002003100 & 0130020000 & 84.3267 \\
\hline 0000020301 & 0302001000 & 0020300001 & 106.7370 \\
\hline 0000300021 & 0301002000 & 0300020001 & 106.7370 \\
\hline 0010020300 & 0100302000 & 0020300010 & 67.9084 \\
\hline 0010300020 & 0102000300 & 0300020010 & 67.9084 \\
\hline 0020300100 & 0010302000 & 0020300100 & 72.8168 \\
\hline 0020100300 & 0012000300 & 0300020100 & 72.8168 \\
\hline 0020301000 & 0000302001 & 0020301000 & 93.5771 \\
\hline 0021000300 & 0002000301 & 0300021000 & 93.5771 \\
\hline 0000020310 & 0001302000 & 0020310000 & 95.4404 \\
\hline 0000310020 & 0002001300 & 0310020000 & 95.4404 \\
\hline 0000021030 & 0003012000 & 0021030000 & 19.1707 \\
\hline 0001030020 & 0002003010 & 1030020000 & 19.1707 \\
\hline
\end{tabular}




\begin{tabular}{|c|c|c|c|}
\hline 0000021300 & 0000312000 & 0021300000 & 89.0103 \\
\hline 0001300020 & 0002000310 & 1300020000 & 89.0103 \\
\hline 0000023001 & 0032001000 & 0023000001 & 101.1280 \\
\hline 0003000021 & 0031002000 & 3000020001 & 101.1280 \\
\hline 0010023000 & 0032000100 & 0023000010 & 60.1248 \\
\hline 0013000020 & 0030102000 & 3000020010 & 60.1248 \\
\hline 0023000100 & 0010032000 & 0023000100 & 64.7753 \\
\hline 0020103000 & 0012000030 & 3000020100 & 64.7753 \\
\hline 0023001000 & 0000032001 & 0023001000 & 102.2230 \\
\hline 0021003000 & 0002000031 & 3000021000 & 102.2230 \\
\hline 0000023010 & 0001032000 & 0023010000 & 68.7238 \\
\hline 0003010020 & 0002001030 & 3010020000 & 68.7238 \\
\hline 0000023100 & 0000132000 & 0023100000 & 89.7537 \\
\hline 0003100020 & 0002000130 & 3100020000 & 89.7537 \\
\hline 0000030102 & 0103002000 & 0030100002 & 74.1229 \\
\hline 0000100032 & 0102003000 & 0100030002 & 74.1229 \\
\hline 0020030100 & 0103000200 & 0030100020 & 68.6854 \\
\hline 0020100030 & 0100203000 & 0100030020 & 68.6854 \\
\hline 0030100200 & 0020103000 & 0030100200 & 67.8232 \\
\hline 0030200100 & 0023000100 & 0100030200 & 67.8232 \\
\hline 0030102000 & 0000103002 & 0030102000 & 74.2781 \\
\hline 0032000100 & 0003000102 & 0100032000 & 74.2781 \\
\hline 0000030120 & 0002103000 & 0030120000 & 87.7501 \\
\hline 0000120030 & 0003002100 & 0120030000 & 87.7501 \\
\hline 0000030201 & 0203001000 & 0030200001 & 115.1771 \\
\hline 0000200031 & 0201003000 & 0200030001 & 115.1771 \\
\hline 0010030200 & 0100203000 & 0030200010 & 69.6407 \\
\hline 0010200030 & 0103000200 & 0200030010 & 69.6407 \\
\hline 0030200100 & 0010203000 & 0030200100 & 73.9921 \\
\hline 0030100200 & 0013000200 & 0200030100 & 73.9921 \\
\hline 0030201000 & 0000203001 & 0030201000 & 93.5512 \\
\hline 0031000200 & 0003000201 & 0200031000 & 93.5512 \\
\hline 0000030210 & 0001203000 & 0030210000 & 92.6256 \\
\hline 0000210030 & 0003001200 & 0210030000 & 92.6256 \\
\hline 0000031020 & 0002013000 & 0031020000 & 64.7017 \\
\hline 0001020030 & 0003002010 & 1020030000 & 64.7017 \\
\hline 0000031200 & 0000213000 & 0031200000 & 90.2811 \\
\hline 0001200030 & 0003000210 & 1200030000 & 90.2811 \\
\hline 0000032010 & 0001023000 & 0032010000 & 72.9162 \\
\hline 0002010030 & 0003001020 & 2010030000 & 72.9162 \\
\hline 0000032100 & 0000123000 & 0032100000 & 82.7925 \\
\hline 0002100030 & 0003000120 & 2100030000 & 82.7925 \\
\hline 0000100203 & 0103000200 & 0100200003 & 84.0781 \\
\hline 0000200103 & 0100203000 & 0200100003 & 84.0781 \\
\hline 0030100200 & 0100300200 & 0100200030 & 69.9906 \\
\hline 0030200100 & 0100200300 & 0200100030 & 69.9906 \\
\hline 0000100230 & 0003200100 & 0100230000 & 80.4333 \\
\hline 0000230100 & 0000103200 & 0230100000 & 80.4333 \\
\hline 0000100302 & 0102000300 & 0100300002 & 82.9985 \\
\hline 0000300102 & 0100302000 & 0300100002 & 82.9985 \\
\hline
\end{tabular}




$\begin{array}{lllll}0020100300 & 0100200300 & 0100300020 & 74.1851 \\ 0020300100 & 0100300200 & 0300100020 & 74.1851 \\ 0000100320 & 0002300100 & 0100320000 & 36.9899 \\ 0000320100 & 0000102300 & 0320100000 & 36.9899 \\ 0000102030 & 0003020100 & 0102030000 & 74.1934 \\ 0002030100 & 0000103020 & 2030100000 & 74.1934 \\ 0000120300 & 0000302100 & 0120300000 & 98.5900 \\ 0000300120 & 0002100300 & 0300120000 & 98.5900 \\ 0000130200 & 0000203100 & 0130200000 & 97.9272 \\ 0000200130 & 0003100200 & 0200130000 & 97.9272 \\ 0000200301 & 0201000300 & 0200300001 & 90.1220 \\ 0000300201 & 0200301000 & 0300200001 & 90.1220 \\ 0010200300 & 0100300200 & 0200300010 & 74.0034 \\ 0010300200 & 0100200300 & 0300200010 & 74.0034 \\ 0000200310 & 0001300200 & 0200310000 & 84.0433 \\ 0000310200 & 0000201300 & 0310200000 & 84.0433 \\ 0000201030 & 0003010200 & 0201030000 & 71.0970 \\ 0001030200 & 0000203010 & 1030200000 & 71.0970 \\ 0000210300 & 0000301200 & 0210300000 & 83.5683 \\ 0000300210 & 0001200300 & 0300210000 & 83.5683 \\ 0000301020 & 0002010300 & 0301020000 & 67.5030 \\ 0001020300 & 0000302010 & 1020300000 & 67.5030\end{array}$

bullvalene $4 \mathbf{h}$ : Single point DFT calculations at B3LYP-D3BJ/Def2-TZVPPD/CPCM (chloroform)

Vertex list:

\begin{tabular}{lrl} 
isomer & \multicolumn{3}{c}{ energy } \\
\hdashline 0000000122 & 70.5207 \\
0000000212 & 100.5381 \\
0000000221 & 87.1216 \\
0000001022 & 66.8677 \\
0000001202 & 82.4315 \\
0000001220 & 72.5610 \\
0000002012 & 64.2671 \\
0000002021 & 62.7146 \\
0000002102 & 73.5237 \\
0000002120 & 78.7516 \\
0000002201 & 73.6237 \\
0000002210 & 61.7207 \\
0000010022 & 75.6629 \\
0000020012 & 75.6629 \\
0000010202 & 51.8824 \\
0000200012 & 51.8824 \\
0000010220 & 47.1924 \\
0000220010 & 47.1924 \\
0000012002 & 65.0189 \\
0002000012 & 65.0189
\end{tabular}




\begin{tabular}{|c|c|}
\hline 0000012020 & 20.9509 \\
\hline 0002020010 & 20.9509 \\
\hline 0000012200 & 50.0741 \\
\hline 0002200010 & 50.0741 \\
\hline 0000020021 & 79.7668 \\
\hline 0000020102 & 62.5647 \\
\hline 0000100022 & 62.5647 \\
\hline 0000020120 & 29.2654 \\
\hline 0000120020 & 29.2654 \\
\hline 0000020201 & 49.6138 \\
\hline 0000200021 & 49.6138 \\
\hline 0000020210 & 31.9494 \\
\hline 0000210020 & 31.9494 \\
\hline 0000021002 & 66.8619 \\
\hline 0001000022 & 66.8619 \\
\hline 0000021020 & 10.1701 \\
\hline 0001020020 & 10.1701 \\
\hline 0000021200 & 36.4790 \\
\hline 0001200020 & 36.4790 \\
\hline 0000022001 & 65.4595 \\
\hline 0002000021 & 65.4595 \\
\hline 0000022010 & 22.5768 \\
\hline 0002010020 & 22.5768 \\
\hline 0000022100 & 31.8095 \\
\hline 0002100020 & 31.8095 \\
\hline 0000100202 & 59.9141 \\
\hline 0000200102 & 59.9141 \\
\hline 0000100220 & 53.6789 \\
\hline 0000220100 & 53.6789 \\
\hline 0000102002 & 54.8517 \\
\hline 0002000102 & 54.8517 \\
\hline 0000102020 & 12.4745 \\
\hline 0002020100 & 12.4745 \\
\hline 0000102200 & 53.5341 \\
\hline 0002200100 & 53.5341 \\
\hline 0000120200 & 36.3876 \\
\hline 0000200120 & 36.3876 \\
\hline 0000122000 & 35.4657 \\
\hline 0002000120 & 35.4657 \\
\hline 0000200201 & 52.2215 \\
\hline 0000200210 & 40.2287 \\
\hline 0000210200 & 40.2287 \\
\hline 0000201002 & 63.5672 \\
\hline 0001000202 & 63.5672 \\
\hline 0000201020 & 30.9280 \\
\hline 0001020200 & 30.9280 \\
\hline 0000201200 & 57.3699 \\
\hline 0001200200 & 57.3699 \\
\hline 0000202001 & 51.0039 \\
\hline 0002000201 & 51.0039 \\
\hline
\end{tabular}




\begin{tabular}{|c|c|}
\hline 0000202010 & 27.1854 \\
\hline 0002010200 & 27.1854 \\
\hline 0000202100 & 50.2291 \\
\hline 0002100200 & 50.2291 \\
\hline 0000212000 & 38.5177 \\
\hline 0002000210 & 38.5177 \\
\hline 0000221000 & 58.1933 \\
\hline 0001000220 & 58.1933 \\
\hline 0001002002 & 82.6455 \\
\hline 0002001002 & 82.6455 \\
\hline 0001002020 & 50.1350 \\
\hline 0002021000 & 50.1350 \\
\hline 0001002200 & 80.6604 \\
\hline 0002201000 & 80.6604 \\
\hline 0001022000 & 53.7681 \\
\hline 0002001020 & 53.7681 \\
\hline 0001202000 & 74.5263 \\
\hline 0002001200 & 74.5263 \\
\hline 0002002001 & 74.0866 \\
\hline 0002002010 & 49.8777 \\
\hline 0002012000 & 49.8777 \\
\hline 0002002100 & 73.0932 \\
\hline 0002102000 & 73.0932 \\
\hline 0010020020 & 0.0000 \\
\hline 0010020200 & 21.7102 \\
\hline 0010200020 & 21.7102 \\
\hline 0010022000 & 23.7964 \\
\hline 0012000020 & 23.7964 \\
\hline 0010200200 & 29.5635 \\
\hline 0010202000 & 28.3336 \\
\hline 0012000200 & 28.3336 \\
\hline 0012002000 & 51.3245 \\
\hline 0020020100 & 6.9996 \\
\hline 0020021000 & 34.9436 \\
\hline 0020100200 & 27.5914 \\
\hline 0020200100 & 27.5914 \\
\hline 0020102000 & 27.6857 \\
\hline 0022000100 & 27.6857 \\
\hline 0020201000 & 30.8792 \\
\hline 0021000200 & 30.8792 \\
\hline 0021002000 & 40.0313 \\
\hline 0022001000 & 40.0313 \\
\hline 0100200200 & 35.1186 \\
\hline 0100202000 & 31.7004 \\
\hline 0102000200 & 31.7004 \\
\hline 0102002000 & 53.3441 \\
\hline 0200201000 & 38.2038 \\
\hline 0201002000 & 58.5192 \\
\hline 0202001000 & 58.5192 \\
\hline 1002002000 & 98.0810 \\
\hline
\end{tabular}


Edge list:

\begin{tabular}{|c|c|c|c|}
\hline isomer1 & ner2 & eTS energy & \\
\hline 0000000122 & 0000002210 & 0000000122 & 122.6962 \\
\hline 0000000212 & 0000002120 & 0000000212 & 135.2784 \\
\hline 0000000221 & 0000001220 & 0000000221 & 141.8592 \\
\hline 0000001022 & 0000002201 & 0000001022 & 133.4565 \\
\hline 0000001202 & 0000002021 & 0000001202 & 145.8237 \\
\hline 0000002012 & 0000002102 & 0000002012 & 138.7627 \\
\hline 0000010022 & 0002201000 & 0000010022 & 130.2125 \\
\hline 0000020012 & 0001002200 & 0010000022 & 130.2125 \\
\hline 0000010202 & 0002021000 & 0000010202 & 114.9308 \\
\hline 0000200012 & 0001002020 & 0010000202 & 114.9308 \\
\hline 0000010220 & 0000221000 & 0000010220 & 103.5431 \\
\hline 0000220010 & 0001000220 & 0010000220 & 103.5431 \\
\hline 0000012002 & 0002001002 & 0000012002 & 134.3192 \\
\hline 0002000012 & 0001002002 & 0010002002 & 134.3192 \\
\hline 0000012020 & 0000201002 & 0000012020 & 105.9771 \\
\hline 0002020010 & 0001000202 & 0010002020 & 105.9771 \\
\hline 0000012200 & 0000021002 & 0000012200 & 116.6001 \\
\hline 0002200010 & 0001000022 & 0010002200 & 116.6001 \\
\hline 0000020012 & 0002102000 & 0000020012 & 111.9005 \\
\hline 0000010022 & 0002002100 & 0020000012 & 111.9005 \\
\hline 0000020021 & 0001202000 & 0000020021 & 124.9258 \\
\hline 0000020021 & 0002001200 & 0020000021 & 124.9258 \\
\hline 0000020102 & 0002012000 & 0000020102 & 95.2427 \\
\hline 0000100022 & 0002002010 & 0020000102 & 95.2427 \\
\hline 0000020120 & 0000212000 & 0000020120 & 70.5023 \\
\hline 0000120020 & 0002000210 & 0020000120 & 70.5023 \\
\hline 0000020201 & 0001022000 & 0000020201 & 100.5227 \\
\hline 0000200021 & 0002001020 & 0020000201 & 100.5227 \\
\hline 0000020210 & 0000122000 & 0000020210 & 62.1332 \\
\hline 0000210020 & 0002000120 & 0020000210 & 62.1332 \\
\hline 0000021002 & 0002002001 & 0000021002 & 115.6668 \\
\hline 0001000022 & 0002002001 & 0020001002 & 115.6668 \\
\hline 0000021020 & 0000202001 & 0000021020 & 76.1860 \\
\hline 0001020020 & 0002000201 & 0020001020 & 76.1860 \\
\hline 0000021200 & 0000022001 & 0000021200 & 99.1348 \\
\hline 0001200020 & 0002000021 & 0020001200 & 99.1348 \\
\hline 0000022001 & 0001002002 & 0000022001 & 155.3536 \\
\hline 0002000021 & 0002001002 & 0020002001 & 155.3536 \\
\hline 0000022010 & 0000102002 & 0000022010 & 80.0764 \\
\hline 0002010020 & 0002000102 & 0020002010 & 80.0764 \\
\hline 0000022100 & 0000012002 & 0000022100 & 93.9174 \\
\hline 0002100020 & 0002000012 & 0020002100 & 93.9174 \\
\hline 0000100022 & 0002200100 & 0000100022 & 125.2113 \\
\hline
\end{tabular}




\begin{tabular}{|c|c|c|c|}
\hline 0000020102 & 0000102200 & 0100000022 & 125.2113 \\
\hline 0000100202 & 0002020100 & 0000100202 & 112.1508 \\
\hline 0000200102 & 0000102020 & 0100000202 & 112.1508 \\
\hline 0000100220 & 0000220100 & 0000100220 & 111.5408 \\
\hline 0000220100 & 0000100220 & 0100000220 & 111.5408 \\
\hline 0000102002 & 0002000102 & 0000102002 & 130.0881 \\
\hline 0002000102 & 0000102002 & 0100002002 & 130.0881 \\
\hline 0000000122 & 0002002100 & 0000120002 & 112.6987 \\
\hline 0000000122 & 0002102000 & 0120000002 & 112.6987 \\
\hline 0000120020 & 0000202100 & 0000120020 & 82.5924 \\
\hline 0000020120 & 0002100200 & 0120000020 & 82.5924 \\
\hline 0000120200 & 0000022100 & 0000120200 & 80.2138 \\
\hline 0000200120 & 0002100020 & 0120000200 & 80.2138 \\
\hline 0000122000 & 0000002102 & 0000122000 & 103.6992 \\
\hline 0002000120 & 0000002102 & 0120002000 & 103.6992 \\
\hline 0000200012 & 0002100200 & 0000200012 & 111.3288 \\
\hline 0000010202 & 0000202100 & 0200000012 & 111.3288 \\
\hline 0000200021 & 0001200200 & 0000200021 & 115.6643 \\
\hline 0000020201 & 0000201200 & 0200000021 & 115.6643 \\
\hline 0000200102 & 0002010200 & 0000200102 & 97.5309 \\
\hline 0000100202 & 0000202010 & 0200000102 & 97.5309 \\
\hline 0000200120 & 0000210200 & 0000200120 & 83.1850 \\
\hline 0000120200 & 0000200210 & 0200000120 & 83.1850 \\
\hline 0000200201 & 0001020200 & 0000200201 & 92.8797 \\
\hline 0000200201 & 0000201020 & 0200000201 & 92.8797 \\
\hline 0000201002 & 0002000201 & 0000201002 & 114.8950 \\
\hline 0001000202 & 0000202001 & 0200001002 & 114.8950 \\
\hline 0000000212 & 0002001200 & 0000210002 & 144.9391 \\
\hline 0000000212 & 0001202000 & 0210000002 & 144.9391 \\
\hline 0000210020 & 0000201200 & 0000210020 & 93.3360 \\
\hline 0000020210 & 0001200200 & 0210000020 & 93.3360 \\
\hline 0000210200 & 0000021200 & 0000210200 & 92.9379 \\
\hline 0000200210 & 0001200020 & 0210000200 & 92.9379 \\
\hline 0000212000 & 0000001202 & 0000212000 & 118.0405 \\
\hline 0002000210 & 0000001202 & 0210002000 & 118.0405 \\
\hline 0000000221 & 0001002200 & 0000220001 & 126.9731 \\
\hline 0000000221 & 0002201000 & 0220000001 & 126.9731 \\
\hline 0000220010 & 0000102200 & 0000220010 & 83.9012 \\
\hline 0000010220 & 0002200100 & 0220000010 & 83.9012 \\
\hline 0000220100 & 0000012200 & 0000220100 & 84.0699 \\
\hline 0000100220 & 0002200010 & 0220000100 & 84.0699 \\
\hline 0000221000 & 0000002201 & 0000221000 & 104.0864 \\
\hline 0001000220 & 0000002201 & 0220001000 & 104.0864 \\
\hline 0000001022 & 0002002010 & 0001020002 & 98.4206 \\
\hline 0000001022 & 0002012000 & 1020000002 & 98.4206 \\
\hline 0001020020 & 0000202010 & 0001020020 & 53.0785 \\
\hline 0000021020 & 0002010200 & 1020000020 & 53.0785 \\
\hline 0001020200 & 0000022010 & 0001020200 & 58.7581 \\
\hline 0000201020 & 0002010020 & 1020000200 & 58.7581 \\
\hline 0001022000 & 0000002012 & 0001022000 & 88.0958 \\
\hline
\end{tabular}




\begin{tabular}{|c|c|c|c|}
\hline 0002001020 & 0000002012 & 1020002000 & 88.0958 \\
\hline 0000001220 & 0000002210 & 0001220000 & 104.3697 \\
\hline 0000001220 & 0000002210 & 1220000000 & 104.3697 \\
\hline 0000002021 & 0001002020 & 0002020001 & 81.6091 \\
\hline 0000002021 & 0002021000 & 2020000001 & 81.6091 \\
\hline 0002020010 & 0000102020 & 0002020010 & 32.1633 \\
\hline 0000012020 & 0002020100 & 2020000010 & 32.1633 \\
\hline 0000002120 & 0000002120 & 0002120000 & 97.0548 \\
\hline 0000002120 & 0000002120 & 2120000000 & 97.0548 \\
\hline 0000010022 & 1002002000 & 0010020002 & 129.9693 \\
\hline 0000020012 & 1002002000 & 0020010002 & 129.9693 \\
\hline 0010020020 & 0202001000 & 0010020020 & 69.7951 \\
\hline 0010020020 & 0201002000 & 0020010020 & 69.7951 \\
\hline 0010020200 & 0022001000 & 0010020200 & 69.0987 \\
\hline 0010200020 & 0021002000 & 0020010200 & 69.0987 \\
\hline 0010022000 & 0002001002 & 0010022000 & 94.5326 \\
\hline 0012000020 & 0001002002 & 0020012000 & 94.5326 \\
\hline 0000010202 & 0201002000 & 0010200002 & 107.9057 \\
\hline 0000200012 & 0202001000 & 0200010002 & 107.9057 \\
\hline 0010200020 & 0200201000 & 0010200020 & 76.0780 \\
\hline 0010020200 & 0200201000 & 0200010020 & 76.0780 \\
\hline 0010200200 & 0020201000 & 0010200200 & 75.3196 \\
\hline 0010200200 & 0021000200 & 0200010200 & 75.3196 \\
\hline 0010202000 & 0000201002 & 0010202000 & 99.3606 \\
\hline 0012000200 & 0001000202 & 0200012000 & 99.3606 \\
\hline 0000010220 & 0002201000 & 0010220000 & 94.7230 \\
\hline 0000220010 & 0001002200 & 0220010000 & 94.7230 \\
\hline 0000012002 & 0021002000 & 0012000002 & 91.6941 \\
\hline 0002000012 & 0022001000 & 2000010002 & 91.6941 \\
\hline 0012000020 & 0021000200 & 0012000020 & 59.5127 \\
\hline 0010022000 & 0020201000 & 2000010020 & 59.5127 \\
\hline 0012000200 & 0020021000 & 0012000200 & 59.3657 \\
\hline 0010202000 & 0020021000 & 2000010200 & 59.3657 \\
\hline 0012002000 & 0000021002 & 0012002000 & 99.1860 \\
\hline 0012002000 & 0001000022 & 2000012000 & 99.1860 \\
\hline 0000012020 & 0002021000 & 0012020000 & 48.0502 \\
\hline 0002020010 & 0001002020 & 2020010000 & 48.0502 \\
\hline 0000012200 & 0000221000 & 0012200000 & 86.6312 \\
\hline 0002200010 & 0001000220 & 2200010000 & 86.6312 \\
\hline 0000020021 & 1002002000 & 0020020001 & 116.2823 \\
\hline 0010020020 & 0102002000 & 0020020010 & 63.2615 \\
\hline 0020020100 & 0012002000 & 0020020100 & 62.2693 \\
\hline 0020021000 & 0002002001 & 0020021000 & 83.3756 \\
\hline 0000020102 & 0102002000 & 0020100002 & 102.5039 \\
\hline 0000100022 & 0102002000 & 0100020002 & 102.5039 \\
\hline 0020020100 & 0102000200 & 0020100020 & 62.3760 \\
\hline 0020020100 & 0100202000 & 0100020020 & 62.3760 \\
\hline 0020100200 & 0020102000 & 0020100200 & 62.8266 \\
\hline 0020200100 & 0022000100 & 0100020200 & 62.8266 \\
\hline 0020102000 & 0000102002 & 0020102000 & 87.4479 \\
\hline
\end{tabular}




$\begin{array}{lllll}0022000100 & 0002000102 & 0100022000 & 87.4479 \\ 0000020120 & 0002102000 & 0020120000 & 83.8304 \\ 0000120020 & 0002002100 & 0120020000 & 83.8304 \\ 0000020201 & 0202001000 & 0020200001 & 97.6623 \\ 0000200021 & 0201002000 & 0200020001 & 97.6623 \\ 0010020200 & 0100202000 & 0020200010 & 55.5182 \\ 0010200020 & 0102000200 & 0200020010 & 55.5182 \\ 0020200100 & 0010202000 & 0020200100 & 55.6188 \\ 0020100200 & 0012000200 & 0200020100 & 55.6188 \\ 0020201000 & 0000202001 & 0020201000 & 74.3050 \\ 0021000200 & 0002000201 & 0200021000 & 74.3050 \\ 0000020210 & 0001202000 & 0020210000 & 88.0817 \\ 0000210020 & 0002001200 & 0210020000 & 88.0817 \\ 0000021020 & 0002012000 & 0021020000 & 60.9814 \\ 0001020020 & 0002002010 & 1020020000 & 60.9814 \\ 0000021200 & 0000212000 & 0021200000 & 73.6543 \\ 0001200020 & 0002000210 & 1200020000 & 73.6543 \\ 0000022001 & 0022001000 & 0022000001 & 90.8160 \\ 0002000021 & 0021002000 & 2000020001 & 90.8160 \\ 0010022000 & 0022000100 & 0022000010 & 42.5795 \\ 0012000020 & 0020102000 & 2000020010 & 42.5795 \\ 0000022010 & 0001022000 & 0022010000 & 59.1505 \\ 0002010020 & 0002001020 & 2010020000 & 59.1505 \\ 0000022100 & 0000122000 & 0022100000 & 66.9155 \\ 0002100020 & 0002000120 & 2100020000 & 66.9155 \\ 0000100202 & 0102000200 & 0100200002 & 105.3107 \\ 0000200102 & 0100202000 & 0200100002 & 105.3107 \\ 0020100200 & 0100200200 & 0100200020 & 81.7397 \\ 0020200100 & 0100200200 & 0200100020 & 81.7397 \\ 0000100220 & 0002200100 & 0100220000 & 89.7473 \\ 0000220100 & 0000102200 & 0220100000 & 89.7473 \\ 0000102020 & 0002020100 & 0102020000 & 39.6002 \\ 0002020100 & 0000102020 & 2020100000 & 39.6002 \\ 0000120200 & 0000202100 & 0120200000 & 78.0956 \\ 0000200120 & 0002100200 & 0200120000 & 78.0956 \\ 0000200201 & 0200201000 & 0200200001 & 87.2708 \\ 0010200200 & 0100200200 & 0200200010 & 71.7462 \\ 0000200210 & 0001200200 & 0200210000 & 89.2436 \\ 0000210200 & 0000201200 & 0210200000 & 89.2436 \\ 0000201020 & 0002010200 & 0201020000 & 57.3401 \\ 0001020200 & 0000202010 & 1020200000 & 57.3401\end{array}$

bullvalene 4i: Single point DFT calculations at B3LYP-D3BJ/Def2-TZVPPD/CPCM (chloroform)

Vertex list:

isomer energy

$0000000123 \quad 73.2358$ 


\begin{tabular}{|c|c|}
\hline 0000000132 & 79.4457 \\
\hline 0000000213 & 85.9857 \\
\hline 0000000231 & 80.2818 \\
\hline 0000000312 & 97.2179 \\
\hline 0000000321 & 83.4095 \\
\hline 0000001023 & 46.1087 \\
\hline 0000001032 & 63.0732 \\
\hline 0000001203 & 61.8160 \\
\hline 0000001230 & 70.1721 \\
\hline 0000001302 & 79.6598 \\
\hline 0000001320 & 61.0653 \\
\hline 0000002013 & 49.5751 \\
\hline 0000002031 & 65.6479 \\
\hline 0000002103 & 50.6294 \\
\hline 0000002130 & 71.7174 \\
\hline 0000002301 & 56.8089 \\
\hline 0000002310 & 63.1807 \\
\hline 0000003012 & 62.1113 \\
\hline 0000003021 & 67.0011 \\
\hline 0000003102 & 80.2093 \\
\hline 0000003120 & 96.7579 \\
\hline 0000003201 & 61.6584 \\
\hline 0000003210 & 72.4956 \\
\hline 0000010023 & 53.5776 \\
\hline 0000020013 & 53.5776 \\
\hline 0000010032 & 70.1331 \\
\hline 0000030012 & 70.1331 \\
\hline 0000010203 & 35.7372 \\
\hline 0000200013 & 35.7372 \\
\hline 0000010230 & 29.7376 \\
\hline 0000230010 & 29.7376 \\
\hline 0000010302 & 56.3829 \\
\hline 0000300012 & 56.3829 \\
\hline 0000010320 & 19.8172 \\
\hline 0000320010 & 19.8172 \\
\hline 0000012003 & 50.9914 \\
\hline 0002000013 & 50.9914 \\
\hline 0000012030 & 20.3384 \\
\hline 0002030010 & 20.3384 \\
\hline 0000012300 & 30.7356 \\
\hline 0002300010 & 30.7356 \\
\hline 0000013002 & 64.3932 \\
\hline 0003000012 & 64.3932 \\
\hline 0000013020 & 5.0153 \\
\hline 0003020010 & 5.0153 \\
\hline 0000013200 & 37.4540 \\
\hline 0003200010 & 37.4540 \\
\hline 0000020031 & 77.0905 \\
\hline 0000030021 & 77.0905 \\
\hline 0000020103 & 38.1487 \\
\hline
\end{tabular}




\begin{tabular}{|c|c|}
\hline 0000100023 & 38.1487 \\
\hline 0000020130 & 27.5766 \\
\hline 0000130020 & 27.5766 \\
\hline 0000020301 & 56.6787 \\
\hline 0000300021 & 56.6787 \\
\hline 0000020310 & 18.5941 \\
\hline 0000310020 & 18.5941 \\
\hline 0000021003 & 46.198 \\
\hline 0001000023 & 46.198 \\
\hline 0000021030 & 7.8851 \\
\hline 0001030020 & 7.8851 \\
\hline 0000021300 & 36.1879 \\
\hline 0001300020 & 36.1879 \\
\hline 0000023001 & 63.5357 \\
\hline 0003000021 & 63.5357 \\
\hline 0000023010 & 6.5431 \\
\hline 0003010020 & 6.5431 \\
\hline 0000023100 & 33.6250 \\
\hline 0003100020 & 33.6250 \\
\hline 0000030102 & 56.9532 \\
\hline 0000100032 & 56.9532 \\
\hline 0000030120 & 29.4085 \\
\hline 0000120030 & 29.408 \\
\hline 0000030201 & 53.906 \\
\hline 0000200031 & 53.906 \\
\hline 0000030210 & 30.3577 \\
\hline 0000210030 & 30.3577 \\
\hline 0000031002 & 64.445 \\
\hline 0001000032 & 64.4453 \\
\hline 0000031020 & 22.700 \\
\hline 0001020030 & 22.700 \\
\hline 0000031200 & 49.854 \\
\hline 0001200030 & 49.854 \\
\hline 0000032001 & 66.229 \\
\hline 0002000031 & 66.2298 \\
\hline 0000032010 & 21.2846 \\
\hline 0002010030 & 21.2846 \\
\hline 0000032100 & 39.1217 \\
\hline 0002100030 & 39.121 \\
\hline 0000100203 & 24.6398 \\
\hline 0000200103 & 24.6398 \\
\hline 0000100230 & 30.216 \\
\hline 0000230100 & 30.216 \\
\hline 0000100302 & 50.595 \\
\hline 0000300102 & 50.595 \\
\hline 0000100320 & 26.467 \\
\hline 0000320100 & 26.467 \\
\hline 0000102003 & 37.976 \\
\hline 0002000103 & 37.976 \\
\hline 0000102030 & 23.094 \\
\hline
\end{tabular}




\begin{tabular}{|c|c|}
\hline 0002030100 & 23.0941 \\
\hline 0000102300 & 36.7025 \\
\hline 0002300100 & 36.7025 \\
\hline 0000103002 & 57.6248 \\
\hline 0003000102 & 57.6248 \\
\hline 0000103020 & 14.3817 \\
\hline 0003020100 & 14.3817 \\
\hline 0000103200 & 46.1938 \\
\hline 0003200100 & 46.1938 \\
\hline 0000120300 & 25.2370 \\
\hline 0000300120 & 25.2370 \\
\hline 0000123000 & 34.1587 \\
\hline 0003000120 & 34.1587 \\
\hline 0000130200 & 31.7346 \\
\hline 0000200130 & 31.7346 \\
\hline 0000132000 & 40.8926 \\
\hline 0002000130 & 40.8926 \\
\hline 0000200301 & 35.9576 \\
\hline 0000300201 & 35.9576 \\
\hline 0000200310 & 27.1466 \\
\hline 0000310200 & 27.1466 \\
\hline 0000201003 & 30.0454 \\
\hline 0001000203 & 30.0454 \\
\hline 0000201030 & 16.3635 \\
\hline 0001030200 & 16.3635 \\
\hline 0000201300 & 40.7080 \\
\hline 0001300200 & 40.7080 \\
\hline 0000203001 & 51.3835 \\
\hline 0003000201 & 51.3835 \\
\hline 0000203010 & 16.9568 \\
\hline 0003010200 & 16.9568 \\
\hline 0000203100 & 38.9173 \\
\hline 0003100200 & 38.9173 \\
\hline 0000210300 & 31.4334 \\
\hline 0000300210 & 31.4334 \\
\hline 0000213000 & 36.1707 \\
\hline 0003000210 & 36.1707 \\
\hline 0000231000 & 36.9006 \\
\hline 0001000230 & 36.9006 \\
\hline 0000301002 & 56.4745 \\
\hline 0001000302 & 56.4745 \\
\hline 0000301020 & 7.3828 \\
\hline 0001020300 & 7.3828 \\
\hline 0000301200 & 47.1952 \\
\hline 0001200300 & 47.1952 \\
\hline 0000302001 & 44.0346 \\
\hline 0002000301 & 44.0346 \\
\hline 0000302010 & 18.8009 \\
\hline 0002010300 & 18.8009 \\
\hline 0000302100 & 43.5525 \\
\hline
\end{tabular}




\begin{tabular}{|c|c|}
\hline 0002100300 & 43.5525 \\
\hline 0000312000 & 39.6005 \\
\hline 0002000310 & 39.6005 \\
\hline 0000321000 & 29.1765 \\
\hline 0001000320 & 29.1765 \\
\hline 0001002003 & 64.2449 \\
\hline 0002001003 & 64.2449 \\
\hline 0001002030 & 52.9075 \\
\hline 0002031000 & 52.9075 \\
\hline 0001002300 & 67.4156 \\
\hline 0002301000 & 67.4156 \\
\hline 0001003002 & 81.943 \\
\hline 0003001002 & 81.943 \\
\hline 0001003020 & 30.9482 \\
\hline 0003021000 & 30.9482 \\
\hline 0001003200 & 78.0488 \\
\hline 0003201000 & 78.0488 \\
\hline 0001023000 & 36.2815 \\
\hline 0003001020 & 36.2815 \\
\hline 0001032000 & 49.282 \\
\hline 0002001030 & 49.282 \\
\hline 0001203000 & 76.9636 \\
\hline 0003001200 & 76.9636 \\
\hline 0001302000 & $79.704 \varepsilon$ \\
\hline 0002001300 & 79.7048 \\
\hline 0002003001 & 66.9023 \\
\hline 0003002001 & 66.9023 \\
\hline 0002003010 & 40.240 \\
\hline 0003012000 & 40.2400 \\
\hline 0002003100 & 67.181 \\
\hline 0003102000 & 67.181 \\
\hline 0002013000 & 40.965 \\
\hline 0003002010 & 40.965 \\
\hline 0002103000 & 62.1688 \\
\hline 0003002100 & 62.168 \\
\hline 0010020030 & 0.0000 \\
\hline 0010030020 & 0.0000 \\
\hline 0010020300 & 0.5444 \\
\hline 0010300020 & 0.5444 \\
\hline 0010023000 & 7.8392 \\
\hline 0013000020 & 7.8392 \\
\hline 0010030200 & 9.4463 \\
\hline 0010200030 & 9.4463 \\
\hline 0010032000 & 21.0338 \\
\hline 0012000030 & 21.033 \\
\hline 0010200300 & 8.0531 \\
\hline 0010300200 & 8.0531 \\
\hline 0010203000 & 20.261 \\
\hline 001300020 & 20.261 \\
\hline 001030200 & 20.494 \\
\hline
\end{tabular}




$\begin{array}{ll}0012000300 & 20.4947 \\ 0012003000 & 44.0374 \\ 0013002000 & 44.0374 \\ 0020030100 & 2.8975 \\ 0020100030 & 2.8975 \\ 0020031000 & 24.2453 \\ 0021000030 & 24.2453 \\ 0020100300 & 6.2289 \\ 0020300100 & 6.2289 \\ 0020103000 & 11.9807 \\ 0023000100 & 11.9807 \\ 0020301000 & 9.4390 \\ 0021000300 & 9.4390 \\ 0021003000 & 35.9460 \\ 0023001000 & 35.9460 \\ 0030100200 & 10.6404 \\ 0030200100 & 10.6404 \\ 0030102000 & 21.4619 \\ 0032000100 & 21.4619 \\ 0030201000 & 22.6577 \\ 0031000200 & 22.6577 \\ 0031002000 & 51.2788 \\ 0032001000 & 51.2788 \\ 0100200300 & 14.8678 \\ 0100300200 & 14.8678 \\ 0100203000 & 20.9535 \\ 0103000200 & 20.9535 \\ 0100302000 & 26.5325 \\ 0102000300 & 26.5325 \\ 0102003000 & 46.5933 \\ 0103002000 & 46.5933 \\ 0200301000 & 17.6113 \\ 0201000300 & 17.6113 \\ 0201003000 & 34.1486 \\ 0203001000 & 34.1486 \\ 0301002000 & 46.4233 \\ 0302001000 & 46.4233 \\ 1002003000 & 84.3614 \\ 1003002000 & 84.3614\end{array}$

bullvalene 4i OTBS --> OTMS analogue: Single point DFT calculations at B3LYP-D3BJ/Def2-TZVPPD/CPCM (chloroform)

Vertex list:

isomer energy

$0000000123 \quad 76.9055$

$0000000132 \quad 82.1107$

$0000000213 \quad 93.0330$

$0000000231 \quad 82.0205$ 


\begin{tabular}{|c|c|}
\hline 0000000312 & 100.5043 \\
\hline 0000000321 & 78.7370 \\
\hline 0000001023 & 49.5785 \\
\hline 0000001032 & 65.2671 \\
\hline 0000001203 & 65.8755 \\
\hline 0000001230 & 74.0325 \\
\hline 0000001302 & 81.8106 \\
\hline 0000001320 & 63.3109 \\
\hline 0000002013 & 57.7040 \\
\hline 0000002031 & 65.5407 \\
\hline 0000002103 & 56.8021 \\
\hline 0000002130 & 74.6261 \\
\hline 0000002301 & 57.6773 \\
\hline 0000002310 & 61.0405 \\
\hline 0000003012 & 64.4824 \\
\hline 0000003021 & 65.3620 \\
\hline 0000003102 & 82.6203 \\
\hline 0000003120 & 101.1169 \\
\hline 0000003201 & 63.3723 \\
\hline 0000003210 & 68.4104 \\
\hline 0000010023 & 54.5981 \\
\hline 0000020013 & 54.5981 \\
\hline 0000010032 & 69.8285 \\
\hline 0000030012 & 69.8285 \\
\hline 0000010203 & 42.9743 \\
\hline 0000200013 & 42.9743 \\
\hline 0000010230 & 30.1142 \\
\hline 0000230010 & 30.1142 \\
\hline 0000010302 & 57.1884 \\
\hline 0000300012 & 57.1884 \\
\hline 0000010320 & 21.5813 \\
\hline 0000320010 & 21.5813 \\
\hline 0000012003 & 52.5534 \\
\hline 0002000013 & 52.5534 \\
\hline 0000012030 & 24.0288 \\
\hline 0002030010 & 24.0288 \\
\hline 0000012300 & 32.6614 \\
\hline 0002300010 & 32.6614 \\
\hline 0000013002 & 66.1220 \\
\hline 0003000012 & 66.1220 \\
\hline 0000013020 & 6.2286 \\
\hline 0003020010 & 6.2286 \\
\hline 0000013200 & 39.0688 \\
\hline 0003200010 & 39.0688 \\
\hline 0000020031 & 85.1018 \\
\hline 0000030021 & 85.1018 \\
\hline 0000020103 & 48.6278 \\
\hline 0000100023 & 48.6278 \\
\hline 0000020130 & 29.0112 \\
\hline 0000130020 & 29.0112 \\
\hline
\end{tabular}




\begin{tabular}{|c|c|}
\hline 0000020301 & 57.3372 \\
\hline 0000300021 & 57.3372 \\
\hline 0000020310 & 22.1475 \\
\hline 0000310020 & 22.1475 \\
\hline 0000021003 & 49.3507 \\
\hline 0001000023 & 49.3507 \\
\hline 0000021030 & 12.9141 \\
\hline 0001030020 & 12.9141 \\
\hline 0000021300 & 37.1972 \\
\hline 0001300020 & 37.1972 \\
\hline 0000023001 & 64.3400 \\
\hline 0003000021 & 64.3400 \\
\hline 0000023010 & 5.3696 \\
\hline 0003010020 & 5.3696 \\
\hline 0000023100 & 36.676 \\
\hline 0003100020 & 36.6764 \\
\hline 0000030102 & 59.8310 \\
\hline 0000100032 & 59.8310 \\
\hline 0000030120 & $36.026 \varsigma$ \\
\hline 0000120030 & 36.026 \\
\hline 0000030201 & 56.0307 \\
\hline 0000200031 & 56.0307 \\
\hline 0000030210 & 29.732 \\
\hline 0000210030 & 29.732 \\
\hline 0000031002 & $67.227 \varepsilon$ \\
\hline 0001000032 & $67.227 \varepsilon$ \\
\hline 0000031020 & 17.3378 \\
\hline 0001020030 & $17.337 \varepsilon$ \\
\hline 0000031200 & 51.9435 \\
\hline 0001200030 & 51.9435 \\
\hline 0000032001 & 68.310 \\
\hline 0002000031 & 68.310 \\
\hline 0000032010 & 23.4467 \\
\hline 0002010030 & 23.4467 \\
\hline 0000032100 & 42.600 \\
\hline 0002100030 & 42.600 \\
\hline 0000100203 & 28.502 \\
\hline 0000200103 & 28.502 \\
\hline 0000100230 & 32.618 \\
\hline 0000230100 & 32.618 \\
\hline 0000100302 & 54.122 \\
\hline 0000300102 & 54.122 \\
\hline 0000100320 & 28.141 \\
\hline 0000320100 & 28.1410 \\
\hline 0000102003 & 38.150 \\
\hline 0002000103 & 38.150 \\
\hline 0000102030 & 24.726 \\
\hline 0002030100 & 24.726 \\
\hline 000010230 & 40.307 \\
\hline 000230010 & 40.307 \\
\hline
\end{tabular}




\begin{tabular}{|c|c|}
\hline 0000103002 & 58.4405 \\
\hline 0003000102 & 58.4405 \\
\hline 0000103020 & 13.3782 \\
\hline 0003020100 & 13.3782 \\
\hline 0000103200 & 45.2978 \\
\hline 0003200100 & 45.2978 \\
\hline 0000120300 & 30.7197 \\
\hline 0000300120 & 30.7197 \\
\hline 0000123000 & 37.5455 \\
\hline 0003000120 & 37.5455 \\
\hline 0000130200 & 33.4330 \\
\hline 0000200130 & 33.4330 \\
\hline 0000132000 & 45.3587 \\
\hline 0002000130 & 45.3587 \\
\hline 0000200301 & 32.0145 \\
\hline 0000300201 & 32.0145 \\
\hline 0000200310 & 30.3900 \\
\hline 0000310200 & 30.3900 \\
\hline 0000201003 & 34.1032 \\
\hline 0001000203 & 34.1032 \\
\hline 0000201030 & 17.1023 \\
\hline 0001030200 & 17.1023 \\
\hline 0000201300 & 43.5736 \\
\hline 0001300200 & 43.5736 \\
\hline 0000203001 & 37.2237 \\
\hline 0003000201 & 37.2237 \\
\hline 0000203010 & 11.5692 \\
\hline 0003010200 & 11.5692 \\
\hline 0000203100 & 42.4187 \\
\hline 0003100200 & 42.4187 \\
\hline 0000210300 & 32.1490 \\
\hline 0000300210 & 32.1490 \\
\hline 0000213000 & 36.8689 \\
\hline 0003000210 & 36.8689 \\
\hline 0000231000 & 40.8757 \\
\hline 0001000230 & 40.8757 \\
\hline 0000301002 & 57.2984 \\
\hline 0001000302 & 57.2984 \\
\hline 0000301020 & 10.1896 \\
\hline 0001020300 & 10.1896 \\
\hline 0000301200 & 50.2472 \\
\hline 0001200300 & 50.2472 \\
\hline 0000302001 & 45.2539 \\
\hline 0002000301 & 45.2539 \\
\hline 0000302010 & 19.4565 \\
\hline 0002010300 & 19.4565 \\
\hline 0000302100 & 46.5486 \\
\hline 0002100300 & 46.5486 \\
\hline 0000312000 & 44.1966 \\
\hline 0002000310 & 44.1966 \\
\hline
\end{tabular}




\begin{tabular}{|c|c|}
\hline 0000321000 & 31.7063 \\
\hline 0001000320 & 31.7063 \\
\hline 0001002003 & 66.4538 \\
\hline 0002001003 & 66.4538 \\
\hline 0001002030 & 51.1462 \\
\hline 0002031000 & 51.1462 \\
\hline 0001002300 & 65.8681 \\
\hline 0002301000 & 65.868 \\
\hline 0001003002 & 84.746 \\
\hline 0003001002 & 84.746 \\
\hline 0001003020 & 38.7508 \\
\hline 0003021000 & 38.7508 \\
\hline 0001003200 & 68.8 \\
\hline 0003201000 & 68.84 \\
\hline 0001023000 & 38.84 \\
\hline 0003001020 & 38.848 \\
\hline 0001032000 & 48.806 \\
\hline 0002001030 & 48.8062 \\
\hline 0001203000 & 79.1062 \\
\hline 0003001200 & 79.1062 \\
\hline 0001302000 & 92.3107 \\
\hline 0002001300 & 92.3107 \\
\hline 0002003001 & 65.2140 \\
\hline 0003002001 & 65.2140 \\
\hline 0002003010 & 38.0838 \\
\hline 0003012000 & 38.0838 \\
\hline 0002003100 & 71.300 \\
\hline 0003102000 & 71.300 \\
\hline 0002013000 & $40.682 \varepsilon$ \\
\hline 0003002010 & 40.6828 \\
\hline 0002103000 & 66.284 \\
\hline 0003002100 & 66.284 \\
\hline 0010020030 & 2.8098 \\
\hline 0010030020 & 2.8098 \\
\hline 0010020300 & 0.0000 \\
\hline 0010300020 & 0.0000 \\
\hline 0010023000 & 7.5612 \\
\hline 0013000020 & 7.5612 \\
\hline 0010030200 & 11.913 \\
\hline 0010200030 & 11.913 \\
\hline 0010032000 & 24.732 \\
\hline 0012000030 & 24.732 \\
\hline 0010200300 & 8.5050 \\
\hline 0010300200 & 8.5050 \\
\hline 0010203000 & 14.730 \\
\hline 0013000200 & 14.730 \\
\hline 0010302000 & 21.667 \\
\hline 0012000300 & 21.667 \\
\hline 0012003000 & 42.831 \\
\hline 00130 & 42.831 \\
\hline
\end{tabular}




$\begin{array}{lll}0020030100 & 6.5076 \\ 0020100030 & 6.5076 \\ 0020031000 & 19.9135 \\ 0021000030 & 19.9135 \\ 0020100300 & 9.8879 \\ 0020300100 & 9.8879 \\ 0020103000 & 13.8805 \\ 0023000100 & 13.8805 \\ 0020301000 & 12.3970 \\ 0021000300 & 12.3970 \\ 0021003000 & 38.1589 \\ 0023001000 & 38.1589 \\ 0030100200 & 14.5198 \\ 0030200100 & 14.5198 \\ 0030102000 & 25.2852 \\ 0032000100 & 25.2852 \\ 0030201000 & 20.0157 \\ 0031000200 & 20.0157 \\ 0031002000 & 51.1263 \\ 0032001000 & 51.1263 \\ 0100200300 & 16.7282 \\ 0100300200 & 16.7282 \\ 0100203000 & 19.0309 \\ 0103000200 & 19.0309 \\ 0100302000 & 27.2545 \\ 0102000300 & 27.2545 \\ 0102003000 & 46.0304 \\ 0103002000 & 46.0304 \\ 0200301000 & 20.6030 \\ 0201000300 & 20.6030 \\ 0201003000 & 45.1409 \\ 0203001000 & 45.1409 \\ 0301002000 & 46.8024 \\ 0302001000 & 46.8024 \\ 1002003000 & 85.8109 \\ 1003002000 & 85.8109\end{array}$

Edge list:

\begin{tabular}{|c|c|c|c|}
\hline isomer1 & er2 & TS energy & \\
\hline 0000000123 & 0000003210 & 0000000123 & 127.0460 \\
\hline 0000000132 & 0000002310 & 0000000132 & 129.5079 \\
\hline 0000000213 & 0000003120 & 0000000213 & 154.5096 \\
\hline 0000000231 & 0000001320 & 0000000231 & 133.4571 \\
\hline 0000000312 & 0000002130 & 0000000312 & 137.6549 \\
\hline 0000000321 & 0000001230 & 0000000321 & 136.9429 \\
\hline 0000001023 & 0000003201 & 0000001023 & 116.1624 \\
\hline
\end{tabular}




\begin{tabular}{|c|c|c|c|}
\hline 0000001032 & 0000002301 & 0000001032 & 122.5648 \\
\hline 0000001203 & 0000003021 & 0000001203 & 128.4465 \\
\hline 0000001302 & 0000002031 & 0000001302 & 143.7649 \\
\hline 0000002013 & 0000003102 & 0000002013 & 127.3280 \\
\hline 0000002103 & 0000003012 & 0000002103 & 120.5242 \\
\hline 0000010023 & 0003201000 & 0000010023 & 111.3514 \\
\hline 0000020013 & 0001003200 & 0010000023 & 111.3514 \\
\hline 0000010032 & 0002301000 & 0000010032 & 120.3690 \\
\hline 0000030012 & 0001002300 & 0010000032 & 120.3690 \\
\hline 0000010203 & 0003021000 & 0000010203 & 83.9807 \\
\hline 0000200013 & 0001003020 & 0010000203 & 83.9807 \\
\hline 0000010230 & 0000321000 & 0000010230 & 73.6985 \\
\hline 0000230010 & 0001000320 & 0010000230 & 73.6985 \\
\hline 0000010302 & 0002031000 & 0000010302 & 110.4656 \\
\hline 0000300012 & 0001002030 & 0010000302 & 110.4656 \\
\hline 0000010320 & 0000231000 & 0000010320 & 79.9287 \\
\hline 0000320010 & 0001000230 & 0010000320 & 79.9287 \\
\hline 0000012003 & 0003001002 & 0000012003 & 127.6537 \\
\hline 0002000013 & 0001003002 & 0010002003 & 127.6537 \\
\hline 0000012030 & 0000301002 & 0000012030 & 93.9696 \\
\hline 0002030010 & 0001000302 & 0010002030 & 93.9696 \\
\hline 0000012300 & 0000031002 & 0000012300 & 107.3723 \\
\hline 0002300010 & 0001000032 & 0010002300 & 107.3723 \\
\hline 0000013002 & 0002001003 & 0000013002 & 120.7468 \\
\hline 0003000012 & 0001002003 & 0010003002 & 120.7468 \\
\hline 0000013020 & 0000201003 & 0000013020 & 71.7640 \\
\hline 0003020010 & 0001000203 & 0010003020 & 71.7640 \\
\hline 0000013200 & 0000021003 & 0000013200 & 99.4007 \\
\hline 0003200010 & 0001000023 & 0010003200 & 99.4007 \\
\hline 0000020013 & 0003102000 & 0000020013 & 110.5130 \\
\hline 0000010023 & 0002003100 & 0020000013 & 110.5130 \\
\hline 0000020031 & 0001302000 & 0000020031 & 131.3402 \\
\hline 0000030021 & 0002001300 & 0020000031 & 131.3402 \\
\hline 0000020103 & 0003012000 & 0000020103 & 83.2135 \\
\hline 0000100023 & 0002003010 & 0020000103 & 83.2135 \\
\hline 0000020130 & 0000312000 & 0000020130 & 69.0102 \\
\hline 0000130020 & 0002000310 & 0020000130 & 69.0102 \\
\hline 0000020301 & 0001032000 & 0000020301 & 94.1377 \\
\hline 0000300021 & 0002001030 & 0020000301 & 94.1377 \\
\hline 0000020310 & 0000132000 & 0000020310 & 69.3853 \\
\hline 0000310020 & 0002000130 & 0020000310 & 69.3853 \\
\hline 0000021003 & 0003002001 & 0000021003 & 110.8827 \\
\hline 0001000023 & 0002003001 & 0020001003 & 110.8827 \\
\hline 0000021030 & 0000302001 & 0000021030 & 69.9295 \\
\hline 0001030020 & 0002000301 & 0020001030 & 69.9295 \\
\hline 0000021300 & 0000032001 & 0000021300 & 99.2921 \\
\hline 0001300020 & 0002000031 & 0020001300 & 99.2921 \\
\hline 0000023001 & 0001002003 & 0000023001 & 108.8517 \\
\hline 0003000021 & 0002001003 & 0020003001 & 108.8517 \\
\hline 0000023010 & 0000102003 & 0000023010 & 63.6001 \\
\hline
\end{tabular}




\begin{tabular}{|c|c|c|c|}
\hline 0003010020 & 0002000103 & 0020003010 & 63.6001 \\
\hline 0000023100 & 0000012003 & 0000023100 & 81.1934 \\
\hline 0003100020 & 0002000013 & 0020003100 & 81.1934 \\
\hline 0000030012 & 0002103000 & 0000030012 & 123.4691 \\
\hline 0000010032 & 0003002100 & 0030000012 & 123.4691 \\
\hline 0000030021 & 0001203000 & 0000030021 & 141.8122 \\
\hline 0000020031 & 0003001200 & 0030000021 & 141.8122 \\
\hline 0000030102 & 0002013000 & 0000030102 & 105.3962 \\
\hline 0000100032 & 0003002010 & 0030000102 & 105.3962 \\
\hline 0000030120 & 0000213000 & 0000030120 & 85.7739 \\
\hline 0000120030 & 0003000210 & 0030000120 & 85.7739 \\
\hline 0000030201 & 0001023000 & 0000030201 & 103.0372 \\
\hline 0000200031 & 0003001020 & 0030000201 & 103.0372 \\
\hline 0000030210 & 0000123000 & 0000030210 & 84.1868 \\
\hline 0000210030 & 0003000120 & 0030000210 & 84.1868 \\
\hline 0000031002 & 0002003001 & 0000031002 & 125.6304 \\
\hline 0001000032 & 0003002001 & 0030001002 & 125.6304 \\
\hline 0000031020 & 0000203001 & 0000031020 & 84.0397 \\
\hline 0001020030 & 0003000201 & 0030001020 & 84.0397 \\
\hline 0000031200 & 0000023001 & 0000031200 & 121.0818 \\
\hline 0001200030 & 0003000021 & 0030001200 & 121.0818 \\
\hline 0000032001 & 0001003002 & 0000032001 & 138.8039 \\
\hline 0002000031 & 0003001002 & 0030002001 & 138.8039 \\
\hline 0000032010 & 0000103002 & 0000032010 & 102.2680 \\
\hline 0002010030 & 0003000102 & 0030002010 & 102.2680 \\
\hline 0000032100 & 0000013002 & 0000032100 & 113.9939 \\
\hline 0002100030 & 0003000012 & 0030002100 & 113.9939 \\
\hline 0000100023 & 0003200100 & 0000100023 & 102.4490 \\
\hline 0000020103 & 0000103200 & 0100000023 & 102.4490 \\
\hline 0000100032 & 0002300100 & 0000100032 & 112.7675 \\
\hline 0000030102 & 0000102300 & 0100000032 & 112.7675 \\
\hline 0000100203 & 0003020100 & 0000100203 & 74.2255 \\
\hline 0000200103 & 0000103020 & 0100000203 & 74.2255 \\
\hline 0000100230 & 0000320100 & 0000100230 & 85.6948 \\
\hline 0000230100 & 0000100320 & 0100000230 & 85.6948 \\
\hline 0000100302 & 0002030100 & 0000100302 & 102.0664 \\
\hline 0000300102 & 0000102030 & 0100000302 & 102.0664 \\
\hline 0000102003 & 0003000102 & 0000102003 & 111.8897 \\
\hline 0002000103 & 0000103002 & 0100002003 & 111.8897 \\
\hline 0000000123 & 0003002100 & 0000120003 & 115.3523 \\
\hline 0000000123 & 0002103000 & 0120000003 & 115.3523 \\
\hline 0000120030 & 0000302100 & 0000120030 & 73.0491 \\
\hline 0000030120 & 0002100300 & 0120000030 & 73.0491 \\
\hline 0000120300 & 0000032100 & 0000120300 & 72.4653 \\
\hline 0000300120 & 0002100030 & 0120000300 & 72.4653 \\
\hline 0000123000 & 0000002103 & 0000123000 & 83.2375 \\
\hline 0003000120 & 0000002103 & 0120003000 & 83.2375 \\
\hline 0000000132 & 0002003100 & 0000130002 & 131.4086 \\
\hline 0000000132 & 0003102000 & 0130000002 & 131.4086 \\
\hline 0000130020 & 0000203100 & 0000130020 & 87.7065 \\
\hline
\end{tabular}




\begin{tabular}{|c|c|c|c|}
\hline 0000020130 & 0003100200 & 0130000020 & 87.7065 \\
\hline 0000130200 & 0000023100 & 0000130200 & 88.5853 \\
\hline 0000200130 & 0003100020 & 0130000200 & 88.5853 \\
\hline 0000132000 & 0000003102 & 0000132000 & 125.3365 \\
\hline 0002000130 & 0000003102 & 0130002000 & 125.3365 \\
\hline 0000200013 & 0003100200 & 0000200013 & 101.4570 \\
\hline 0000010203 & 0000203100 & 0200000013 & 101.4570 \\
\hline 0000200031 & 0001300200 & 0000200031 & 116.0214 \\
\hline 0000030201 & 0000201300 & 0200000031 & 116.0214 \\
\hline 0000200103 & 0003010200 & 0000200103 & 80.2142 \\
\hline 0000100203 & 0000203010 & 0200000103 & 80.2142 \\
\hline 0000200130 & 0000310200 & 0000200130 & 88.4647 \\
\hline 0000130200 & 0000200310 & 0200000130 & 88.4647 \\
\hline 0000200301 & 0001030200 & 0000200301 & 86.5420 \\
\hline 0000300201 & 0000201030 & 0200000301 & 86.5420 \\
\hline 0000201003 & 0003000201 & 0000201003 & 97.9623 \\
\hline 0001000203 & 0000203001 & 0200001003 & 97.9623 \\
\hline 0000000213 & 0003001200 & 0000210003 & 144.3682 \\
\hline 0000000213 & 0001203000 & 0210000003 & 144.3682 \\
\hline 0000210030 & 0000301200 & 0000210030 & 84.2887 \\
\hline 0000030210 & 0001200300 & 0210000030 & 84.2887 \\
\hline 0000210300 & 0000031200 & 0000210300 & 88.1448 \\
\hline 0000300210 & 0001200030 & 0210000300 & 88.1448 \\
\hline 0000213000 & 0000001203 & 0000213000 & 99.3193 \\
\hline 0003000210 & 0000001203 & 0210003000 & 99.3193 \\
\hline 0000000231 & 0001003200 & 0000230001 & 136.7036 \\
\hline 0000000231 & 0003201000 & 0230000001 & 136.7036 \\
\hline 0000230010 & 0000103200 & 0000230010 & 95.6140 \\
\hline 0000010230 & 0003200100 & 0230000010 & 95.6140 \\
\hline 0000230100 & 0000013200 & 0000230100 & 93.3024 \\
\hline 0000100230 & 0003200010 & 0230000100 & 93.3024 \\
\hline 0000231000 & 0000003201 & 0000231000 & 112.7053 \\
\hline 0001000230 & 0000003201 & 0230001000 & 112.7053 \\
\hline 0000300012 & 0002100300 & 0000300012 & 120.0897 \\
\hline 0000010302 & 0000302100 & 0300000012 & 120.0897 \\
\hline 0000300021 & 0001200300 & 0000300021 & 128.2086 \\
\hline 0000020301 & 0000301200 & 0300000021 & 128.2086 \\
\hline 0000300102 & 0002010300 & 0000300102 & 108.1569 \\
\hline 0000100302 & 0000302010 & 0300000102 & 108.1569 \\
\hline 0000300120 & 0000210300 & 0000300120 & 94.4362 \\
\hline 0000120300 & 0000300210 & 0300000120 & 94.4362 \\
\hline 0000300201 & 0001020300 & 0000300201 & 90.8615 \\
\hline 0000200301 & 0000301020 & 0300000201 & 90.8615 \\
\hline 0000301002 & 0002000301 & 0000301002 & 124.9319 \\
\hline 0001000302 & 0000302001 & 0300001002 & 124.9319 \\
\hline 0000000312 & 0002001300 & 0000310002 & 149.5276 \\
\hline 0000000312 & 0001302000 & 0310000002 & 149.5276 \\
\hline 0000310020 & 0000201300 & 0000310020 & 86.8381 \\
\hline 0000020310 & 0001300200 & 0310000020 & 86.8381 \\
\hline 0000310200 & 0000021300 & 0000310200 & 87.0104 \\
\hline
\end{tabular}




\begin{tabular}{|c|c|c|c|}
\hline 0000200310 & 0001300020 & 0310000200 & 87.0104 \\
\hline 0000312000 & 0000001302 & 0000312000 & 123.6757 \\
\hline 0002000310 & 0000001302 & 0310002000 & 123.6757 \\
\hline 0000000321 & 0001002300 & 0000320001 & 136.2433 \\
\hline 0000000321 & 0002301000 & 0320000001 & 136.2433 \\
\hline 0000320010 & 0000102300 & 0000320010 & 71.2752 \\
\hline 0000010320 & 0002300100 & 0320000010 & 71.2752 \\
\hline 0000320100 & 0000012300 & 0000320100 & 71.3553 \\
\hline 0000100320 & 0002300010 & 0320000100 & 71.3553 \\
\hline 0000321000 & 0000002301 & 0000321000 & 92.1758 \\
\hline 0001000320 & 0000002301 & 0320001000 & 92.1758 \\
\hline 0000001023 & 0003002010 & 0001020003 & 89.4515 \\
\hline 0000001023 & 0002013000 & 1020000003 & 89.4515 \\
\hline 0001020030 & 0000302010 & 0001020030 & 53.4368 \\
\hline 0000031020 & 0002010300 & 1020000030 & 53.4368 \\
\hline 0001020300 & 0000032010 & 0001020300 & 47.4370 \\
\hline 0000301020 & 0002010030 & 1020000300 & 47.4370 \\
\hline 0001023000 & 0000002013 & 0001023000 & 56.9431 \\
\hline 0003001020 & 0000002013 & 1020003000 & 56.9431 \\
\hline 0000001032 & 0002003010 & 0001030002 & 106.5581 \\
\hline 0000001032 & 0003012000 & 1030000002 & 106.5581 \\
\hline 0001030020 & 0000203010 & 0001030020 & 61.2156 \\
\hline 0000021030 & 0003010200 & 1030000020 & 61.2156 \\
\hline 0001030200 & 0000023010 & 0001030200 & 60.4938 \\
\hline 0000201030 & 0003010020 & 1030000200 & 60.4938 \\
\hline 0001032000 & 0000003012 & 0001032000 & 111.1581 \\
\hline 0002001030 & 0000003012 & 1030002000 & 111.1581 \\
\hline 0000001230 & 0000003210 & 0001230000 & 124.8971 \\
\hline 0000001230 & 0000003210 & 1230000000 & 124.8971 \\
\hline 0000001320 & 0000002310 & 0001320000 & 92.8100 \\
\hline 0000001320 & 0000002310 & 1320000000 & 92.8100 \\
\hline 0000002031 & 0001003020 & 0002030001 & 91.3759 \\
\hline 0000002031 & 0003021000 & 2030000001 & 91.3759 \\
\hline 0002030010 & 0000103020 & 0002030010 & 51.7122 \\
\hline 0000012030 & 0003020100 & 2030000010 & 51.7122 \\
\hline 0002030100 & 0000013020 & 0002030100 & 50.6930 \\
\hline 0000102030 & 0003020010 & 2030000100 & 50.6930 \\
\hline 0002031000 & 0000003021 & 0002031000 & 99.9893 \\
\hline 0001002030 & 0000003021 & 2030001000 & 99.9893 \\
\hline 0000002130 & 0000003120 & 0002130000 & 122.7690 \\
\hline 0000002130 & 0000003120 & 2130000000 & 122.7690 \\
\hline 0000010023 & 1003002000 & 0010020003 & 104.9078 \\
\hline 0000020013 & 1002003000 & 0020010003 & 104.9078 \\
\hline 0010020030 & 0302001000 & 0010020030 & 60.5255 \\
\hline 0010030020 & 0301002000 & 0020010030 & 60.5255 \\
\hline 0010020300 & 0032001000 & 0010020300 & 65.5769 \\
\hline 0010300020 & 0031002000 & 0020010300 & 65.5769 \\
\hline 0010023000 & 0002001003 & 0010023000 & 76.9406 \\
\hline 0013000020 & 0001002003 & 0020013000 & 76.9406 \\
\hline 0000010032 & 1002003000 & 0010030002 & 132.4682 \\
\hline
\end{tabular}




\begin{tabular}{|c|c|c|c|}
\hline 0000030012 & 1003002000 & 0030010002 & 132.4682 \\
\hline 0010030020 & 0203001000 & 0010030020 & 73.1929 \\
\hline 0010020030 & 0201003000 & 0030010020 & 73.1929 \\
\hline 0010030200 & 0023001000 & 0010030200 & 69.8779 \\
\hline 0010200030 & 0021003000 & 0030010200 & 69.8779 \\
\hline 0010032000 & 0003001002 & 0010032000 & 109.6473 \\
\hline 0012000030 & 0001003002 & 0030012000 & 109.6473 \\
\hline 0000010203 & 0201003000 & 0010200003 & 93.3796 \\
\hline 0000200013 & 0203001000 & 0200010003 & 93.3796 \\
\hline 0010200030 & 0201000300 & 0010200030 & 67.4014 \\
\hline 0010030200 & 0200301000 & 0200010030 & 67.4014 \\
\hline 0010200300 & 0030201000 & 0010200300 & 72.8534 \\
\hline 0010300200 & 0031000200 & 0200010300 & 72.8534 \\
\hline 0010203000 & 0000201003 & 0010203000 & 82.9269 \\
\hline 0013000200 & 0001000203 & 0200013000 & 82.9269 \\
\hline 0000010230 & 0003201000 & 0010230000 & 98.7402 \\
\hline 0000230010 & 0001003200 & 0230010000 & 98.7402 \\
\hline 0000010302 & 0301002000 & 0010300002 & 117.5110 \\
\hline 0000300012 & 0302001000 & 0300010002 & 117.5110 \\
\hline 0010300020 & 0200301000 & 0010300020 & 74.3197 \\
\hline 0010020300 & 0201000300 & 0300010020 & 74.3197 \\
\hline 0010300200 & 0020301000 & 0010300200 & 74.5397 \\
\hline 0010200300 & 0021000300 & 0300010200 & 74.5397 \\
\hline 0010302000 & 0000301002 & 0010302000 & 108.4263 \\
\hline 0012000300 & 0001000302 & 0300012000 & 108.4263 \\
\hline 0000010320 & 0002301000 & 0010320000 & 84.3313 \\
\hline 0000320010 & 0001002300 & 0320010000 & 84.3313 \\
\hline 0000012003 & 0021003000 & 0012000003 & 83.5288 \\
\hline 0002000013 & 0023001000 & 2000010003 & 83.5288 \\
\hline 0012000030 & 0021000300 & 0012000030 & 49.7493 \\
\hline 0010032000 & 0020301000 & 2000010030 & 49.7493 \\
\hline 0012000300 & 0021000030 & 0012000300 & 51.1563 \\
\hline 0010302000 & 0020031000 & 2000010300 & 51.1563 \\
\hline 0012003000 & 0000021003 & 0012003000 & 90.2271 \\
\hline 0013002000 & 0001000023 & 2000013000 & 90.2271 \\
\hline 0000012030 & 0003021000 & 0012030000 & 61.6119 \\
\hline 0002030010 & 0001003020 & 2030010000 & 61.6119 \\
\hline 0000012300 & 0000321000 & 0012300000 & 70.5188 \\
\hline 0002300010 & 0001000320 & 2300010000 & 70.5188 \\
\hline 0000013002 & 0031002000 & 0013000002 & 113.1373 \\
\hline 0003000012 & 0032001000 & 3000010002 & 113.1373 \\
\hline 0013000020 & 0031000200 & 0013000020 & 63.1907 \\
\hline 0010023000 & 0030201000 & 3000010020 & 63.1907 \\
\hline 0013000200 & 0020031000 & 0013000200 & 62.3578 \\
\hline 0010203000 & 0021000030 & 3000010200 & 62.3578 \\
\hline 0013002000 & 0000031002 & 0013002000 & 106.8583 \\
\hline 0012003000 & 0001000032 & 3000012000 & 106.8583 \\
\hline 0000013020 & 0002031000 & 0013020000 & 65.6751 \\
\hline 0003020010 & 0001002030 & 3020010000 & 65.6751 \\
\hline 0000013200 & 0000231000 & 0013200000 & 96.4672 \\
\hline
\end{tabular}




\begin{tabular}{|c|c|c|c|}
\hline 0003200010 & 0001000230 & 3200010000 & 96.4672 \\
\hline 0000020031 & 1003002000 & 0020030001 & 117.0087 \\
\hline 0000030021 & 1002003000 & 0030020001 & 117.0087 \\
\hline 0010020030 & 0103002000 & 0020030010 & 53.5439 \\
\hline 0010030020 & 0102003000 & 0030020010 & 53.5439 \\
\hline 0020030100 & 0013002000 & 0020030100 & 72.3619 \\
\hline 0020100030 & 0012003000 & 0030020100 & 72.3619 \\
\hline 0020031000 & 0003002001 & 0020031000 & 75.8449 \\
\hline 0021000030 & 0002003001 & 0030021000 & 75.8449 \\
\hline 0000020103 & 0102003000 & 0020100003 & 95.5192 \\
\hline 0000100023 & 0103002000 & 0100020003 & 95.5192 \\
\hline 0020100030 & 0102000300 & 0020100030 & 55.9604 \\
\hline 0020030100 & 0100302000 & 0100020030 & 55.9604 \\
\hline 0020100300 & 0030102000 & 0020100300 & 56.5406 \\
\hline 0020300100 & 0032000100 & 0100020300 & 56.5406 \\
\hline 0020103000 & 0000102003 & 0020103000 & 66.0048 \\
\hline 0023000100 & 0002000103 & 0100023000 & 66.0048 \\
\hline 0000020130 & 0003102000 & 0020130000 & 81.5378 \\
\hline 0000130020 & 0002003100 & 0130020000 & 81.5378 \\
\hline 0000020301 & 0302001000 & 0020300001 & 109.6252 \\
\hline 0000300021 & 0301002000 & 0300020001 & 109.6252 \\
\hline 0010020300 & 0100302000 & 0020300010 & 62.2464 \\
\hline 0010300020 & 0102000300 & 0300020010 & 62.2464 \\
\hline 0020300100 & 0010302000 & 0020300100 & 63.4818 \\
\hline 0020100300 & 0012000300 & 0300020100 & 63.4818 \\
\hline 0020301000 & 0000302001 & 0020301000 & 83.9138 \\
\hline 0021000300 & 0002000301 & 0300021000 & 83.9138 \\
\hline 0000020310 & 0001302000 & 0020310000 & 101.4731 \\
\hline 0000310020 & 0002001300 & 0310020000 & 101.4731 \\
\hline 0000021030 & 0003012000 & 0021030000 & 60.8684 \\
\hline 0001030020 & 0002003010 & 1030020000 & 60.8684 \\
\hline 0000021300 & 0000312000 & 0021300000 & 55.3483 \\
\hline 0001300020 & 0002000310 & 1300020000 & 55.3483 \\
\hline 0000023001 & 0032001000 & 0023000001 & 98.2372 \\
\hline 0003000021 & 0031002000 & 3000020001 & 98.2372 \\
\hline 0010023000 & 0032000100 & 0023000010 & 51.6217 \\
\hline 0013000020 & 0030102000 & 3000020010 & 51.6217 \\
\hline 0023000100 & 0010032000 & 0023000100 & 52.9136 \\
\hline 0020103000 & 0012000030 & 3000020100 & 52.9136 \\
\hline 0023001000 & 0000032001 & 0023001000 & 93.2534 \\
\hline 0021003000 & 0002000031 & 3000021000 & 93.2534 \\
\hline 0000023010 & 0001032000 & 0023010000 & 62.2602 \\
\hline 0003010020 & 0002001030 & 3010020000 & 62.2602 \\
\hline 0000023100 & 0000132000 & 0023100000 & 75.8271 \\
\hline 0003100020 & 0002000130 & 3100020000 & 75.8271 \\
\hline 0000030102 & 0103002000 & 0030100002 & 114.6199 \\
\hline 0000100032 & 0102003000 & 0100030002 & 114.6199 \\
\hline 0020030100 & 0103000200 & 0030100020 & 73.1773 \\
\hline 0020100030 & 0100203000 & 0100030020 & 73.1773 \\
\hline 0030100200 & 0020103000 & 0030100200 & 74.1562 \\
\hline
\end{tabular}




\begin{tabular}{|c|c|c|c|}
\hline 0030200100 & 0023000100 & 0100030200 & 74.1562 \\
\hline 0030102000 & 0000103002 & 0030102000 & 110.3220 \\
\hline 0032000100 & 0003000102 & 0100032000 & 110.3220 \\
\hline 0000030120 & 0002103000 & 0030120000 & 81.0765 \\
\hline 0000120030 & 0003002100 & 0120030000 & 81.0765 \\
\hline 0000030201 & 0203001000 & 0030200001 & 111.2055 \\
\hline 0000200031 & 0201003000 & 0200030001 & 111.2055 \\
\hline 0010030200 & 0100203000 & 0030200010 & 74.9668 \\
\hline 0010200030 & 0103000200 & 0200030010 & 74.9668 \\
\hline 0030200100 & 0010203000 & 0030200100 & 74.4486 \\
\hline 0030100200 & 0013000200 & 0200030100 & 74.4486 \\
\hline 0030201000 & 0000203001 & 0030201000 & 94.1467 \\
\hline 0031000200 & 0003000201 & 0200031000 & 94.1467 \\
\hline 0000030210 & 0001203000 & 0030210000 & 102.2673 \\
\hline 0000210030 & 0003001200 & 0210030000 & 102.2673 \\
\hline 0000031020 & 0002013000 & 0031020000 & 52.6612 \\
\hline 0001020030 & 0003002010 & 1020030000 & 52.6612 \\
\hline 0000031200 & 0000213000 & 0031200000 & 89.1777 \\
\hline 0001200030 & 0003000210 & 1200030000 & 89.1777 \\
\hline 0000032010 & 0001023000 & 0032010000 & 60.3014 \\
\hline 0002010030 & 0003001020 & 2010030000 & 60.3014 \\
\hline 0000032100 & 0000123000 & 0032100000 & 79.2160 \\
\hline 0002100030 & 0003000120 & 2100030000 & 79.2160 \\
\hline 0000100203 & 0103000200 & 0100200003 & 83.8368 \\
\hline 0000200103 & 0100203000 & 0200100003 & 83.8368 \\
\hline 0030100200 & 0100300200 & 0100200030 & 75.9244 \\
\hline 0030200100 & 0100200300 & 0200100030 & 75.9244 \\
\hline 0000100230 & 0003200100 & 0100230000 & 100.8485 \\
\hline 0000230100 & 0000103200 & 0230100000 & 100.8485 \\
\hline 0000100302 & 0102000300 & 0100300002 & 120.2374 \\
\hline 0000300102 & 0100302000 & 0300100002 & 120.2374 \\
\hline 0020100300 & 0100200300 & 0100300020 & 82.0846 \\
\hline 0020300100 & 0100300200 & 0300100020 & 82.0846 \\
\hline 0000100320 & 0002300100 & 0100320000 & 82.7777 \\
\hline 0000320100 & 0000102300 & 0320100000 & 82.7777 \\
\hline 0000102030 & 0003020100 & 0102030000 & 61.2529 \\
\hline 0002030100 & 0000103020 & 2030100000 & 61.2529 \\
\hline 0000120300 & 0000302100 & 0120300000 & 89.8567 \\
\hline 0000300120 & 0002100300 & 0300120000 & 89.8567 \\
\hline 0000130200 & 0000203100 & 0130200000 & 100.5371 \\
\hline 0000200130 & 0003100200 & 0200130000 & 100.5371 \\
\hline 0000200301 & 0201000300 & 0200300001 & 96.7386 \\
\hline 0000300201 & 0200301000 & 0300200001 & 96.7386 \\
\hline 0010200300 & 0100300200 & 0200300010 & 80.5723 \\
\hline 0010300200 & 0100200300 & 0300200010 & 80.5723 \\
\hline 0000200310 & 0001300200 & 0200310000 & 95.3099 \\
\hline 0000310200 & 0000201300 & 0310200000 & 95.3099 \\
\hline 0000201030 & 0003010200 & 0201030000 & 75.0792 \\
\hline 0001030200 & 0000203010 & 1030200000 & 75.0792 \\
\hline 0000210300 & 0000301200 & 0210300000 & 99.6166 \\
\hline
\end{tabular}




$\begin{array}{llll}0000300210 & 0001200300 & 0300210000 & 99.6166 \\ 0000301020 & 0002010300 & 0301020000 & 69.4383 \\ 0001020300 & 0000302010 & 1020300000 & 69.4383\end{array}$

bullvalene 6: Single point DFT calculations at B3LYP-D3BJ/Def2-TZVPPD/CPCM (chloroform)

Vertex list:

\begin{tabular}{lrl} 
isomer & energy \\
\hline------- & \\
0000001234 & 90.2288 \\
0000001243 & 89.4717 \\
0000001324 & 83.3111 \\
0000001342 & 100.9560 \\
0000001423 & 93.9095 \\
0000001432 & 108.8653 \\
0000002134 & 106.9529 \\
0000002143 & 102.8610 \\
0000002314 & 109.0369 \\
0000002341 & 98.8742 \\
0000002413 & 110.5348 \\
0000002431 & 98.6260 \\
0000003124 & 113.1355 \\
0000003142 & 136.8392 \\
0000003214 & 108.6667 \\
0000003241 & 94.1334 \\
0000003412 & 119.2169 \\
0000003421 & 99.4493 \\
0000004123 & 112.5875 \\
0000004132 & 122.6683 \\
0000004213 & 107.6938 \\
0000004231 & 100.0603 \\
0000004312 & 127.3793 \\
0000004321 & 94.5119 \\
0000010234 & 75.9911 \\
0000230014 & 75.9911 \\
0000010243 & 64.3167 \\
0000240013 & 64.3167 \\
0000010324 & 63.9055 \\
0000320014 & 63.9055 \\
0000010342 & 91.1399 \\
0000340012 & 91.1399 \\
0000010423 & 63.7211 \\
0000420013 & 63.7211 \\
0000010432 & 94.6050 \\
0000430012 & 94.6050 \\
0000012034 & 47.3934 \\
0002030014 & 47.3934 \\
0000012043 & 53.0026
\end{tabular}




\begin{tabular}{|c|c|}
\hline 0002040013 & 53.0026 \\
\hline 0000012304 & 52.9393 \\
\hline 0002300014 & 52.9393 \\
\hline 0000012340 & 52.0368 \\
\hline 0002340010 & 52.0368 \\
\hline 0000012403 & 60.8761 \\
\hline 0002400013 & 60.8761 \\
\hline 0000012430 & 53.7780 \\
\hline 0002430010 & 53.7780 \\
\hline 0000013024 & 36.6395 \\
\hline 0003020014 & 36.6395 \\
\hline 0000013042 & 68.5481 \\
\hline 0003040012 & 68.5481 \\
\hline 0000013204 & 59.5408 \\
\hline 0003200014 & 59.5408 \\
\hline 0000013240 & 48.6220 \\
\hline 0003240010 & 48.6220 \\
\hline 0000013402 & 72.3739 \\
\hline 0003400012 & 72.3739 \\
\hline 0000013420 & 38.2858 \\
\hline 0003420010 & 38.2858 \\
\hline 0000014023 & 57.1155 \\
\hline 0004020013 & 57.1155 \\
\hline 0000014032 & 70.8071 \\
\hline 0004030012 & 70.8071 \\
\hline 0000014203 & 43.9389 \\
\hline 0004200013 & 43.9389 \\
\hline 0000014230 & 47.9495 \\
\hline 0004230010 & 47.9495 \\
\hline 0000014302 & 62.3351 \\
\hline 0004300012 & 62.3351 \\
\hline 0000014320 & 37.1264 \\
\hline 0004320010 & 37.1264 \\
\hline 0000020134 & 63.3587 \\
\hline 0000130024 & 63.3587 \\
\hline 0000020143 & 77.7527 \\
\hline 0000140023 & 77.7527 \\
\hline 0000020314 & 82.5741 \\
\hline 0000310024 & 82.5741 \\
\hline 0000020341 & 93.4512 \\
\hline 0000340021 & 93.4512 \\
\hline 0000020413 & 91.1089 \\
\hline 0000410023 & 91.1089 \\
\hline 0000020431 & 94.7435 \\
\hline 0000430021 & 94.7435 \\
\hline 0000021034 & 43.3739 \\
\hline 0001030024 & 43.3739 \\
\hline 0000021043 & 52.8270 \\
\hline 0001040023 & 52.8270 \\
\hline 0000021304 & 69.3633 \\
\hline
\end{tabular}




\begin{tabular}{|c|c|}
\hline 0001300024 & 69.3633 \\
\hline 0000021340 & 45.7509 \\
\hline 0001340020 & 45.7509 \\
\hline 0000021403 & 73.8823 \\
\hline 0001400023 & 73.8823 \\
\hline 0000021430 & 48.1959 \\
\hline 0001430020 & 48.1959 \\
\hline 0000023014 & 55.0533 \\
\hline 0003010024 & 55.0533 \\
\hline 0000023041 & 80.9208 \\
\hline 0003040021 & 80.9208 \\
\hline 0000023104 & 71.5864 \\
\hline 0003100024 & 71.5864 \\
\hline 0000023140 & 66.8517 \\
\hline 0003140020 & 66.8517 \\
\hline 0000023401 & 69.3774 \\
\hline 0003400021 & 69.3774 \\
\hline 0000023410 & 38.8053 \\
\hline 0003410020 & 38.8053 \\
\hline 0000024013 & 75.9976 \\
\hline 0004010023 & 75.9976 \\
\hline 0000024031 & 83.5880 \\
\hline 0004030021 & 83.5880 \\
\hline 0000024103 & 66.0345 \\
\hline 0004100023 & 66.0345 \\
\hline 0000024130 & 73.5035 \\
\hline 0004130020 & 73.5035 \\
\hline 0000024301 & 58.3354 \\
\hline 0004300021 & 58.3354 \\
\hline 0000024310 & 31.4485 \\
\hline 0004310020 & 31.4485 \\
\hline 0000030124 & 75.3119 \\
\hline 0000120034 & 75.3119 \\
\hline 0000030142 & 93.1369 \\
\hline 0000140032 & 93.1369 \\
\hline 0000030214 & 86.6069 \\
\hline 0000210034 & 86.6069 \\
\hline 0000030241 & 95.2152 \\
\hline 0000240031 & 95.2152 \\
\hline 0000030412 & 113.9113 \\
\hline 0000410032 & 113.9113 \\
\hline 0000030421 & 89.4868 \\
\hline 0000420031 & 89.4868 \\
\hline 0000031024 & 45.0453 \\
\hline 0001020034 & 45.0453 \\
\hline 0000031042 & 75.0209 \\
\hline 0001040032 & 75.0209 \\
\hline 0000031204 & 53.6471 \\
\hline 0001200034 & 53.6471 \\
\hline 0000031240 & 67.2483 \\
\hline
\end{tabular}




\begin{tabular}{|c|c|}
\hline 0001240030 & 67.2483 \\
\hline 0000031402 & 81.2418 \\
\hline 0001400032 & 81.2418 \\
\hline 0000031420 & 51.8472 \\
\hline 0001420030 & 51.8472 \\
\hline 0000032014 & 47.7997 \\
\hline 0002010034 & 47.7997 \\
\hline 0000032041 & 82.4524 \\
\hline 0002040031 & 82.4524 \\
\hline 0000032104 & 44.7625 \\
\hline 0002100034 & 44.7625 \\
\hline 0000032140 & 70.8947 \\
\hline 0002140030 & 70.8947 \\
\hline 0000032401 & 76.6406 \\
\hline 0002400031 & 76.6406 \\
\hline 0000032410 & 57.8709 \\
\hline 0002410030 & 57.8709 \\
\hline 0000034012 & 73.6561 \\
\hline 0004010032 & 73.6561 \\
\hline 0000034021 & 87.3484 \\
\hline 0004020031 & 87.3484 \\
\hline 0000034102 & 78.7852 \\
\hline 0004100032 & 78.7852 \\
\hline 0000034120 & 88.6506 \\
\hline 0004120030 & 88.6506 \\
\hline 0000034201 & 67.3498 \\
\hline 0004200031 & 67.3498 \\
\hline 0000034210 & 47.6836 \\
\hline 0004210030 & 47.6836 \\
\hline 0000040123 & 78.2595 \\
\hline 0000120043 & 78.2595 \\
\hline 0000040132 & 96.5547 \\
\hline 0000130042 & 96.5547 \\
\hline 0000040213 & 91.2660 \\
\hline 0000210043 & 91.2660 \\
\hline 0000040231 & 91.1734 \\
\hline 0000230041 & 91.1734 \\
\hline 0000040312 & 104.3055 \\
\hline 0000310042 & 104.3055 \\
\hline 0000040321 & 104.6817 \\
\hline 0000320041 & 104.6817 \\
\hline 0000041023 & 54.7968 \\
\hline 0001020043 & 54.7968 \\
\hline 0000041032 & 74.9010 \\
\hline 0001030042 & 74.9010 \\
\hline 0000041203 & 59.9244 \\
\hline 0001200043 & 59.9244 \\
\hline 0000041230 & 62.4004 \\
\hline 0001230040 & 62.4004 \\
\hline 0000041302 & 82.8805 \\
\hline
\end{tabular}




\begin{tabular}{|c|c|}
\hline 0001300042 & 82.8805 \\
\hline 0000041320 & 52.0651 \\
\hline 0001320040 & 52.0651 \\
\hline 0000042013 & 52.1333 \\
\hline 0002010043 & 52.1333 \\
\hline 0000042031 & 82.7663 \\
\hline 0002030041 & 82.7663 \\
\hline 0000042103 & 55.3886 \\
\hline 0002100043 & 55.3886 \\
\hline 0000042130 & 72.2678 \\
\hline 0002130040 & 72.2678 \\
\hline 0000042301 & 73.1566 \\
\hline 0002300041 & 73.1566 \\
\hline 0000042310 & 56.2176 \\
\hline 0002310040 & 56.2176 \\
\hline 0000043012 & 67.772 \\
\hline 0003010042 & 67.772 \\
\hline 0000043021 & 83.214 \\
\hline 0003020041 & 83.2148 \\
\hline 0000043102 & 82.945 \\
\hline 0003100042 & 82.945 \\
\hline 0000043120 & 64.528 \\
\hline 0003120040 & 64.528 \\
\hline 0000043201 & 83.265 \\
\hline 0003200041 & 83.265 \\
\hline 0000043210 & 58.692 \\
\hline 0003210040 & 58.692 \\
\hline 0000100234 & 49.582 \\
\hline 0000230104 & 49.582 \\
\hline 0000100243 & 47.052 \\
\hline 0000240103 & 47.052 \\
\hline 0000100324 & 49.690 \\
\hline 0000320104 & 49.6903 \\
\hline 0000100342 & 68.248 \\
\hline 0000340102 & 68.248 \\
\hline 0000100423 & 53.6686 \\
\hline 0000420103 & 53.6686 \\
\hline 0000100432 & 78.858 \\
\hline 0000430102 & 78.858 \\
\hline 0000102034 & 26.276 \\
\hline 0002030104 & 26.276 \\
\hline 0000102043 & 32.678 \\
\hline 0002040103 & 32.678 \\
\hline 0000102304 & 41.581 \\
\hline 0002300104 & 41.581 \\
\hline 0000102340 & 50.687 \\
\hline 0002340100 & 50.687 \\
\hline 0000102403 & 48.746 \\
\hline 0002400103 & 48.746 \\
\hline 000010243 & 54.796 \\
\hline
\end{tabular}




\begin{tabular}{|c|c|}
\hline 0002430100 & 54.7964 \\
\hline 0000103024 & 48.2782 \\
\hline 0003020104 & 48.2782 \\
\hline 0000103042 & 59.6097 \\
\hline 0003040102 & 59.6097 \\
\hline 0000103204 & 47.9129 \\
\hline 0003200104 & 47.9129 \\
\hline 0000103240 & 52.9416 \\
\hline 0003240100 & 52.94 \\
\hline 0000103402 & 63.132 \\
\hline 0003400102 & 63.132 \\
\hline 0000103420 & 43.59 \\
\hline 0003420100 & $43.594 C$ \\
\hline 0000104023 & 45.6107 \\
\hline 0004020103 & 45.6107 \\
\hline 0000104032 & 63.5355 \\
\hline 0004030102 & 63.535 \\
\hline 0000104203 & 29.47 \\
\hline 0004200103 & 29.47 \\
\hline 0000104230 & 58.168 \\
\hline 0004230100 & $58.168 \varepsilon$ \\
\hline 0000104302 & 54.532 \\
\hline 0004300102 & 54.532 \\
\hline 0000104320 & 52.772 \\
\hline 0004320100 & 52.772 \\
\hline 0000120304 & 48.045 \\
\hline 0000300124 & 48.045 \\
\hline 0000120340 & 31.735 \\
\hline 0000340120 & 31.735 \\
\hline 0000120403 & 71.186 \\
\hline 0000400123 & 71.186 \\
\hline 0000120430 & 40.599 \\
\hline 0000430120 & 40.599 \\
\hline 0000123004 & 54.704 \\
\hline 0003000124 & 54.704 \\
\hline 0000123040 & 26.2566 \\
\hline 0003040120 & 26.256 \\
\hline 0000123400 & 41.788 \\
\hline 0003400120 & 41.788 \\
\hline 0000124003 & 75.546 \\
\hline 0004000123 & 75.546 \\
\hline 0000124030 & 33.313 \\
\hline 0004030120 & 33.313 \\
\hline 0000124300 & 33.907 \\
\hline 0004300120 & 33.907 \\
\hline 0000130204 & 58.591 \\
\hline 0000200134 & 58.591 \\
\hline 0000130240 & 45.510 \\
\hline 0000240130 & 45.510 \\
\hline 0000130402 & 76.942 \\
\hline
\end{tabular}




\begin{tabular}{|c|c|}
\hline 0000400132 & 76.9427 \\
\hline 0000130420 & 57.5977 \\
\hline 0000420130 & 57.5977 \\
\hline 0000132004 & 69.9214 \\
\hline 0002000134 & 69.9214 \\
\hline 0000132040 & 36.6505 \\
\hline 0002040130 & 36.6505 \\
\hline 0000132400 & 53.0533 \\
\hline 0002400130 & 53.0533 \\
\hline 0000134002 & 80.3327 \\
\hline 0004000132 & 80.3327 \\
\hline 0000134020 & 31.2628 \\
\hline 0004020130 & 31.2628 \\
\hline 0000134200 & 38.9722 \\
\hline 0004200130 & 38.9722 \\
\hline 0000140203 & 54.8590 \\
\hline 0000200143 & 54.8590 \\
\hline 0000140230 & 44.8819 \\
\hline 0000230140 & 44.8819 \\
\hline 0000140302 & 74.9141 \\
\hline 0000300142 & 74.9141 \\
\hline 0000140320 & 35.2964 \\
\hline 0000320140 & 35.2964 \\
\hline 0000142003 & 66.9100 \\
\hline 0002000143 & 66.9100 \\
\hline 0000142030 & 29.9226 \\
\hline 0002030140 & 29.9226 \\
\hline 0000142300 & 46.0826 \\
\hline 0002300140 & 46.0826 \\
\hline 0000143002 & 86.6962 \\
\hline 0003000142 & 86.6962 \\
\hline 0000143020 & 21.0469 \\
\hline 0003020140 & 21.0469 \\
\hline 0000143200 & 54.4295 \\
\hline 0003200140 & 54.4295 \\
\hline 0000200314 & 74.8748 \\
\hline 0000310204 & 74.8748 \\
\hline 0000200341 & 62.3759 \\
\hline 0000340201 & 62.3759 \\
\hline 0000200413 & 71.9757 \\
\hline 0000410203 & 71.9757 \\
\hline 0000200431 & 65.6050 \\
\hline 0000430201 & 65.6050 \\
\hline 0000201034 & 17.9137 \\
\hline 0001030204 & 17.9137 \\
\hline 0000201043 & 29.3792 \\
\hline 0001040203 & 29.3792 \\
\hline 0000201304 & 45.7198 \\
\hline 0001300204 & 45.7198 \\
\hline 0000201340 & 51.5954 \\
\hline
\end{tabular}




\begin{tabular}{|c|c|}
\hline 0001340200 & 51.5954 \\
\hline 0000201403 & 49.7508 \\
\hline 0001400203 & 49.7508 \\
\hline 0000201430 & 56.7739 \\
\hline 0001430200 & 56.7739 \\
\hline 0000203014 & 30.4801 \\
\hline 0003010204 & 30.4801 \\
\hline 0000203041 & 51.6341 \\
\hline 0003040201 & 51.6341 \\
\hline 0000203104 & 45.9550 \\
\hline 0003100204 & 45.9550 \\
\hline 0000203140 & 75.0540 \\
\hline 0003140200 & 75.0540 \\
\hline 0000203401 & 42.6847 \\
\hline 0003400201 & 42.6847 \\
\hline 0000203410 & 45.1004 \\
\hline 0003410200 & 45.1004 \\
\hline 0000204013 & 36.9500 \\
\hline 0004010203 & 36.9500 \\
\hline 0000204031 & 51.4821 \\
\hline 0004030201 & 51.4821 \\
\hline 0000204103 & 42.9320 \\
\hline 0004100203 & 42.9320 \\
\hline 0000204130 & 80.6463 \\
\hline 0004130200 & 80.6463 \\
\hline 0000204301 & 34.0731 \\
\hline 0004300201 & 34.0731 \\
\hline 0000204310 & 40.0735 \\
\hline 0004310200 & 40.0735 \\
\hline 0000210304 & 74.0600 \\
\hline 0000300214 & 74.0600 \\
\hline 0000210340 & 39.3510 \\
\hline 0000340210 & 39.3510 \\
\hline 0000210403 & 82.2359 \\
\hline 0000400213 & 82.2359 \\
\hline 0000210430 & 48.7753 \\
\hline 0000430210 & 48.7753 \\
\hline 0000213004 & 82.4977 \\
\hline 0003000214 & 82.4977 \\
\hline 0000213040 & 28.2116 \\
\hline 0003040210 & 28.2116 \\
\hline 0000213400 & 43.2020 \\
\hline 0003400210 & 43.2020 \\
\hline 0000214003 & 84.3454 \\
\hline 0004000213 & 84.3454 \\
\hline 0000214030 & 33.7708 \\
\hline 0004030210 & 33.7708 \\
\hline 0000214300 & 37.4419 \\
\hline 0004300210 & 37.4419 \\
\hline 0000230401 & 77.5896 \\
\hline
\end{tabular}




\begin{tabular}{|c|c|}
\hline 0000400231 & 77.5896 \\
\hline 0000230410 & 49.7914 \\
\hline 0000410230 & 49.7914 \\
\hline 0000231004 & 62.5039 \\
\hline 0001000234 & $62.503 \subseteq$ \\
\hline 0000231040 & 28.7174 \\
\hline 0001040230 & 28.717 \\
\hline 0000231400 & 63.2870 \\
\hline 0001400230 & 63.2870 \\
\hline 0000234001 & 79.8488 \\
\hline 0004000231 & 79.8488 \\
\hline 0000234010 & 26.989 \\
\hline 0004010230 & 26.989 \\
\hline 0000234100 & 57.159 \\
\hline 0004100230 & 57.1598 \\
\hline 0000240301 & 72.595 \\
\hline 0000300241 & 72.595 \\
\hline 0000240310 & 47.550 \\
\hline 0000310240 & 47.5508 \\
\hline 0000241003 & 55.221 \\
\hline 0001000243 & 55.221 \\
\hline 0000241030 & 23.6368 \\
\hline 0001030240 & 23.6368 \\
\hline 0000241300 & 62.071 \\
\hline 0001300240 & 62.0716 \\
\hline 0000243001 & 79.207 \\
\hline 0003000241 & 79.207 \\
\hline 0000243010 & 31.444 \\
\hline 0003010240 & 31.4449 \\
\hline 0000243100 & 55.5459 \\
\hline 0003100240 & 55.545 \\
\hline 0000300412 & 86.442 \\
\hline 0000410302 & 86.442 \\
\hline 0000300421 & 72.8628 \\
\hline 0000420301 & 72.862 \\
\hline 0000301024 & 43.244 \\
\hline 0001020304 & 43.244 \\
\hline 0000301042 & 56.652 \\
\hline 0001040302 & 56.652 \\
\hline 0000301204 & 52.621 \\
\hline 0001200304 & 52.621 \\
\hline 0000301240 & 57.871 \\
\hline 0001240300 & 57.871 \\
\hline 0000301402 & 75.3577 \\
\hline 0001400302 & 75.357 \\
\hline 0000301420 & 53.272 \\
\hline 0001420300 & 53.272 \\
\hline 0000302014 & 39.314 \\
\hline 0002010304 & 39.314 \\
\hline 0000302041 & 61.764 \\
\hline
\end{tabular}




\begin{tabular}{|c|c|}
\hline 0002040301 & 61.7644 \\
\hline 0000302104 & 49.8249 \\
\hline 0002100304 & 49.8249 \\
\hline 0000302140 & 63.6124 \\
\hline 0002140300 & 63.6124 \\
\hline 0000302401 & 54.2074 \\
\hline 0002400301 & 54.2074 \\
\hline 0000302410 & 54.3854 \\
\hline 0002410300 & 54.3854 \\
\hline 0000304012 & 61.1588 \\
\hline 0004010302 & 61.1588 \\
\hline 0000304021 & 60.5983 \\
\hline 0004020301 & 60.5983 \\
\hline 0000304102 & 74.0167 \\
\hline 0004100302 & 74.0167 \\
\hline 0000304120 & 90.7926 \\
\hline 0004120300 & 90.7926 \\
\hline 0000304201 & 37.2825 \\
\hline 0004200301 & 37.2825 \\
\hline 0000304210 & 53.9304 \\
\hline 0004210300 & 53.9304 \\
\hline 0000310402 & 90.3429 \\
\hline 0000400312 & 90.3429 \\
\hline 0000310420 & 41.7296 \\
\hline 0000420310 & 41.7296 \\
\hline 0000312004 & 91.3244 \\
\hline 0002000314 & 91.3244 \\
\hline 0000312040 & 33.5061 \\
\hline 0002040310 & 33.5061 \\
\hline 0000312400 & 60.9822 \\
\hline 0002400310 & 60.9822 \\
\hline 0000314002 & 96.7750 \\
\hline 0004000312 & 96.7750 \\
\hline 0000314020 & 28.0485 \\
\hline 0004020310 & 28.0485 \\
\hline 0000314200 & 40.0605 \\
\hline 0004200310 & 40.0605 \\
\hline 0000320401 & 79.8245 \\
\hline 0000400321 & 79.8245 \\
\hline 0000320410 & 37.9383 \\
\hline 0000410320 & 37.9383 \\
\hline 0000321004 & 55.7448 \\
\hline 0001000324 & 55.7448 \\
\hline 0000321040 & 24.1817 \\
\hline 0001040320 & 24.1817 \\
\hline 0000321400 & 48.4441 \\
\hline 0001400320 & 48.4441 \\
\hline 0000324001 & 78.3736 \\
\hline 0004000321 & 78.3736 \\
\hline 0000324010 & 20.8556 \\
\hline
\end{tabular}




\begin{tabular}{|c|c|}
\hline 0004010320 & 20.8556 \\
\hline 0000324100 & 48.6274 \\
\hline 0004100320 & 48.6274 \\
\hline 0000341002 & 71.5493 \\
\hline 0001000342 & 71.5493 \\
\hline 0000341020 & 15.1314 \\
\hline 0001020340 & 15.1314 \\
\hline 0000341200 & 56.1025 \\
\hline 0001200340 & 56.1025 \\
\hline 0000342001 & 75.7666 \\
\hline 0002000341 & 75.7666 \\
\hline 0000342010 & 33.8469 \\
\hline 0002010340 & 33.8469 \\
\hline 0000342100 & 57.0012 \\
\hline 0002100340 & 57.0012 \\
\hline 0000401023 & 44.5297 \\
\hline 0001020403 & 44.5297 \\
\hline 0000401032 & 60.2362 \\
\hline 0001030402 & 60.2362 \\
\hline 0000401203 & 62.3859 \\
\hline 0001200403 & 62.3859 \\
\hline 0000401230 & 71.8829 \\
\hline 0001230400 & 71.8829 \\
\hline 0000401302 & 74.4071 \\
\hline 0001300402 & 74.4071 \\
\hline 0000401320 & 57.7567 \\
\hline 0001320400 & 57.7567 \\
\hline 0000402013 & 45.8708 \\
\hline 0002010403 & 45.8708 \\
\hline 0000402031 & 61.1126 \\
\hline 0002030401 & 61.1126 \\
\hline 0000402103 & 49.5695 \\
\hline 0002100403 & 49.5695 \\
\hline 0000402130 & 74.8134 \\
\hline 0002130400 & 74.8134 \\
\hline 0000402301 & 52.8145 \\
\hline 0002300401 & 52.8145 \\
\hline 0000402310 & 71.7887 \\
\hline 0002310400 & 71.7887 \\
\hline 0000403012 & 59.0220 \\
\hline 0003010402 & 59.0220 \\
\hline 0000403021 & 60.6323 \\
\hline 0003020401 & 60.6323 \\
\hline 0000403102 & 75.9356 \\
\hline 0003100402 & 75.9356 \\
\hline 0000403120 & 94.3351 \\
\hline 0003120400 & 94.3351 \\
\hline 0000403201 & 58.4642 \\
\hline 0003200401 & 58.4642 \\
\hline 0000403210 & 60.4721 \\
\hline
\end{tabular}




\begin{tabular}{|c|c|}
\hline 0003210400 & 60.4721 \\
\hline 0000412003 & 85.8561 \\
\hline 0002000413 & 85.8561 \\
\hline 0000412030 & 34.1008 \\
\hline 0002030410 & 34.1008 \\
\hline 0000412300 & 47.1809 \\
\hline 0002300410 & 47.1809 \\
\hline 0000413002 & 104.4525 \\
\hline 0003000412 & 104.4525 \\
\hline 0000413020 & 21.8556 \\
\hline 0003020410 & 21.8556 \\
\hline 0000413200 & 53.7553 \\
\hline 0003200410 & 53.7553 \\
\hline 0000421003 & 62.9104 \\
\hline 0001000423 & 62.9104 \\
\hline 0000421030 & 25.6883 \\
\hline 0001030420 & 25.6883 \\
\hline 0000421300 & 58.0780 \\
\hline 0001300420 & 58.0780 \\
\hline 0000423001 & 71.2926 \\
\hline 0003000421 & 71.2926 \\
\hline 0000423010 & 23.9491 \\
\hline 0003010420 & 23.9491 \\
\hline 0000423100 & 51.8713 \\
\hline 0003100420 & 51.8713 \\
\hline 0000431002 & 85.7238 \\
\hline 0001000432 & 85.7238 \\
\hline 0000431020 & 25.5350 \\
\hline 0001020430 & 25.5350 \\
\hline 0000431200 & 65.1388 \\
\hline 0001200430 & 65.1388 \\
\hline 0000432001 & 78.2034 \\
\hline 0002000431 & 78.2034 \\
\hline 0000432010 & 36.2541 \\
\hline 0002010430 & 36.2541 \\
\hline 0000432100 & 56.4050 \\
\hline 0002100430 & 56.4050 \\
\hline 0001002034 & 51.0186 \\
\hline 0002031004 & 51.0186 \\
\hline 0001002043 & 60.9667 \\
\hline 0002041003 & 60.9667 \\
\hline 0001002304 & 71.0891 \\
\hline 0002301004 & 71.0891 \\
\hline 0001002340 & 78.8538 \\
\hline 0002341000 & 78.8538 \\
\hline 0001002403 & 76.6688 \\
\hline 0002401003 & 76.6688 \\
\hline 0001002430 & 87.8281 \\
\hline 0002431000 & 87.8281 \\
\hline 0001003024 & 69.1086 \\
\hline
\end{tabular}




\begin{tabular}{|c|c|}
\hline 0003021004 & 69.1086 \\
\hline 0001003042 & 84.4176 \\
\hline 0003041002 & 84.4176 \\
\hline 0001003204 & 71.9296 \\
\hline 0003201004 & 71.9296 \\
\hline 0001003240 & 77.4054 \\
\hline 0003241000 & 77.4054 \\
\hline 0001003402 & 86.6260 \\
\hline 0003401002 & 86.6260 \\
\hline 0001003420 & 67.5398 \\
\hline 0003421000 & 67.5398 \\
\hline 0001004023 & 65.0624 \\
\hline 0004021003 & 65.0624 \\
\hline 0001004032 & 81.1885 \\
\hline 0004031002 & 81.1885 \\
\hline 0001004203 & 59.2975 \\
\hline 0004201003 & 59.2975 \\
\hline 0001004230 & 83.0645 \\
\hline 0004231000 & 83.0645 \\
\hline 0001004302 & 80.7288 \\
\hline 0004301002 & 80.7288 \\
\hline 0001004320 & 71.3501 \\
\hline 0004321000 & 71.3501 \\
\hline 0001023004 & 71.1747 \\
\hline 0003001024 & 71.1747 \\
\hline 0001023040 & 31.6825 \\
\hline 0003041020 & 31.6825 \\
\hline 0001023400 & 41.0335 \\
\hline 0003401020 & 41.0335 \\
\hline 0001024003 & 64.8374 \\
\hline 0004001023 & 64.8374 \\
\hline 0001024030 & 31.6221 \\
\hline 0004031020 & 31.6221 \\
\hline 0001024300 & 35.5832 \\
\hline 0004301020 & 35.5832 \\
\hline 0001032004 & 49.3476 \\
\hline 0002001034 & 49.3476 \\
\hline 0001032040 & 44.5836 \\
\hline 0002041030 & 44.5836 \\
\hline 0001032400 & 63.8781 \\
\hline 0002401030 & 63.8781 \\
\hline 0001034002 & 81.8634 \\
\hline 0004001032 & 81.8634 \\
\hline 0001034020 & 33.5620 \\
\hline 0004021030 & 33.5620 \\
\hline 0001034200 & 49.2806 \\
\hline 0004201030 & 49.2806 \\
\hline 0001042003 & 59.6329 \\
\hline 0002001043 & 59.6329 \\
\hline 0001042030 & 38.6946 \\
\hline
\end{tabular}




\begin{tabular}{|c|c|}
\hline 0002031040 & 38.6946 \\
\hline 0001042300 & 60.5555 \\
\hline 0002301040 & 60.5555 \\
\hline 0001043002 & 84.5862 \\
\hline 0003001042 & 84.5862 \\
\hline 0001043020 & 32.5644 \\
\hline 0003021040 & 32.5644 \\
\hline 0001043200 & 61.1206 \\
\hline 0003201040 & 61.1206 \\
\hline 0001203004 & 74.2507 \\
\hline 0003001204 & 74.2507 \\
\hline 0001203040 & 62.1550 \\
\hline 0003041200 & 62.1550 \\
\hline 0001203400 & 72.8204 \\
\hline 0003401200 & 72.8204 \\
\hline 0001204003 & 73.3124 \\
\hline 0004001203 & 73.3124 \\
\hline 0001204030 & 63.7983 \\
\hline 0004031200 & 63.7983 \\
\hline 0001204300 & 70.4236 \\
\hline 0004301200 & 70.4236 \\
\hline 0001234000 & 88.8923 \\
\hline 0004001230 & 88.8923 \\
\hline 0001243000 & 94.4122 \\
\hline 0003001240 & 94.4122 \\
\hline 0001302004 & 85.5751 \\
\hline 0002001304 & 85.5751 \\
\hline 0001302040 & 77.7148 \\
\hline 0002041300 & 77.7148 \\
\hline 0001302400 & 101.0026 \\
\hline 0002401300 & 101.0026 \\
\hline 0001304002 & 108.1327 \\
\hline 0004001302 & 108.1327 \\
\hline 0001304020 & 66.9862 \\
\hline 0004021300 & 66.9862 \\
\hline 0001304200 & 80.6538 \\
\hline 0004201300 & 80.6538 \\
\hline 0001324000 & 84.8291 \\
\hline 0004001320 & 84.8291 \\
\hline 0001342000 & 92.9017 \\
\hline 0002001340 & 92.9017 \\
\hline 0001402003 & 90.4014 \\
\hline 0002001403 & 90.4014 \\
\hline 0001402030 & 86.1636 \\
\hline 0002031400 & 86.1636 \\
\hline 0001402300 & 96.5180 \\
\hline 0002301400 & 96.5180 \\
\hline 0001403002 & 109.1501 \\
\hline 0003001402 & 109.1501 \\
\hline 0001403020 & 64.9843 \\
\hline
\end{tabular}




\begin{tabular}{|c|c|}
\hline 0003021400 & 64.9843 \\
\hline 0001403200 & 95.1944 \\
\hline 0003201400 & 95.1944 \\
\hline 0001423000 & 90.7262 \\
\hline 0003001420 & 90.7262 \\
\hline 0001432000 & 93.5088 \\
\hline 0002001430 & 93.5088 \\
\hline 0002003014 & 65.9567 \\
\hline 0003012004 & 65.9567 \\
\hline 0002003041 & 81.6604 \\
\hline 0003042001 & 81.6604 \\
\hline 0002003104 & 73.6669 \\
\hline 0003102004 & 73.6669 \\
\hline 0002003140 & 107.8958 \\
\hline 0003142000 & 107.8958 \\
\hline 0002003401 & 67.2154 \\
\hline 0003402001 & 67.2154 \\
\hline 0002003410 & 74.2169 \\
\hline 0003412000 & 74.2169 \\
\hline 0002004013 & 65.3698 \\
\hline 0004012003 & 65.3698 \\
\hline 0002004031 & 84.4878 \\
\hline 0004032001 & 84.4878 \\
\hline 0002004103 & 77.5723 \\
\hline 0004102003 & 77.5723 \\
\hline 0002004130 & 93.9640 \\
\hline 0004132000 & 93.9640 \\
\hline 0002004301 & 68.9971 \\
\hline 0004302001 & 68.9971 \\
\hline 0002004310 & 73.4437 \\
\hline 0004312000 & 73.4437 \\
\hline 0002013004 & 60.3574 \\
\hline 0003002014 & 60.3574 \\
\hline 0002013040 & 34.6453 \\
\hline 0003042010 & 34.6453 \\
\hline 0002013400 & 48.5546 \\
\hline 0003402010 & 48.5546 \\
\hline 0002014003 & 64.5413 \\
\hline 0004002013 & 64.5413 \\
\hline 0002014030 & 36.2695 \\
\hline 0004032010 & 36.2695 \\
\hline 0002014300 & 33.6108 \\
\hline 0004302010 & 33.6108 \\
\hline 0002034001 & 82.1332 \\
\hline 0004002031 & 82.1332 \\
\hline 0002034010 & 39.1667 \\
\hline 0004012030 & 39.1667 \\
\hline 0002034100 & 64.6977 \\
\hline 0004102030 & 64.6977 \\
\hline 0002043001 & 79.3380 \\
\hline
\end{tabular}




\begin{tabular}{|c|c|}
\hline 0003002041 & 79.3380 \\
\hline 0002043010 & 28.4921 \\
\hline 0003012040 & 28.4921 \\
\hline 0002043100 & 62.6003 \\
\hline 0003102040 & 62.6003 \\
\hline 0002103004 & 69.8905 \\
\hline 0003002104 & 69.8905 \\
\hline 0002103040 & 58.9654 \\
\hline 0003042100 & 58.9654 \\
\hline 0002103400 & 71.6613 \\
\hline 0003402100 & 71.6613 \\
\hline 0002104003 & 71.1888 \\
\hline 0004002103 & 71.1888 \\
\hline 0002104030 & 57.3617 \\
\hline 0004032100 & 57.3617 \\
\hline 0002104300 & 74.9827 \\
\hline 0004302100 & 74.9827 \\
\hline 0002134000 & 95.4483 \\
\hline 0004002130 & 95.4483 \\
\hline 0002143000 & 95.1598 \\
\hline 0003002140 & 95.1598 \\
\hline 0002304001 & 73.3985 \\
\hline 0004002301 & 73.3985 \\
\hline 0002304010 & 47.9761 \\
\hline 0004012300 & 47.9761 \\
\hline 0002304100 & 78.7402 \\
\hline 0004102300 & 78.7402 \\
\hline 0002314000 & 75.6774 \\
\hline 0004002310 & 75.6774 \\
\hline 0002403001 & 82.6725 \\
\hline 0003002401 & 82.6725 \\
\hline 0002403010 & 58.7313 \\
\hline 0003012400 & 58.7313 \\
\hline 0002403100 & 82.7782 \\
\hline 0003102400 & 82.7782 \\
\hline 0002413000 & 82.8986 \\
\hline 0003002410 & 82.8986 \\
\hline 0003004012 & 67.3373 \\
\hline 0004013002 & 67.3373 \\
\hline 0003004021 & 63.3956 \\
\hline 0004023001 & 63.3956 \\
\hline 0003004102 & 100.4058 \\
\hline 0004103002 & 100.4058 \\
\hline 0003004120 & 86.1922 \\
\hline 0004123000 & 86.1922 \\
\hline 0003004201 & 66.4296 \\
\hline 0004203001 & 66.4296 \\
\hline 0003004210 & 73.4939 \\
\hline 0004213000 & 73.4939 \\
\hline 0003014002 & 65.6553 \\
\hline
\end{tabular}




\begin{tabular}{|c|c|}
\hline 0004003012 & 65.6553 \\
\hline 0003014020 & 3.2023 \\
\hline 0004023010 & 3.2023 \\
\hline 0003014200 & 40.4303 \\
\hline 0004203010 & 40.4303 \\
\hline 0003024001 & 66.1094 \\
\hline 0004003021 & 66.1094 \\
\hline 0003024010 & 8.7749 \\
\hline 0004013020 & 8.7749 \\
\hline 0003024100 & 51.9240 \\
\hline 0004103020 & 51.9240 \\
\hline 0003104002 & 100.3961 \\
\hline 0004003102 & 100.3961 \\
\hline 0003104020 & 56.0264 \\
\hline 0004023100 & 56.0264 \\
\hline 0003104200 & 74.8390 \\
\hline 0004203100 & 74.8390 \\
\hline 0003124000 & 79.7375 \\
\hline 0004003120 & 79.7375 \\
\hline 0003204001 & 67.4297 \\
\hline 0004003201 & 67.4297 \\
\hline 0003204010 & 43.4204 \\
\hline 0004013200 & 43.4204 \\
\hline 0003204100 & 89.3057 \\
\hline 0004103200 & 89.3057 \\
\hline 0003214000 & 74.7938 \\
\hline 0004003210 & 74.7938 \\
\hline 0010020034 & 62.6509 \\
\hline 0010030024 & 62.6509 \\
\hline 0010020043 & 62.7181 \\
\hline 0010040023 & 62.7181 \\
\hline 0010020304 & 57.7268 \\
\hline 0010300024 & 57.7268 \\
\hline 0010020340 & 4.5784 \\
\hline 0010340020 & 4.5784 \\
\hline 0010020403 & 62.4421 \\
\hline 0010400023 & 62.4421 \\
\hline 0010020430 & 12.7809 \\
\hline 0010430020 & 12.7809 \\
\hline 0010023004 & 40.6227 \\
\hline 0013000024 & 40.6227 \\
\hline 0010023040 & 6.6792 \\
\hline 0013040020 & 6.6792 \\
\hline 0010023400 & 13.3052 \\
\hline 0013400020 & 13.3052 \\
\hline 0010024003 & 53.3434 \\
\hline 0014000023 & 53.3434 \\
\hline 0010024030 & 0.2258 \\
\hline 0014030020 & 0.2258 \\
\hline 0010024300 & 0.0000 \\
\hline
\end{tabular}




\begin{tabular}{|c|c|}
\hline 0014300020 & 0.0000 \\
\hline 0010030042 & 87.0974 \\
\hline 0010040032 & 87.0974 \\
\hline 0010030204 & 32.5790 \\
\hline 0010200034 & 32.5790 \\
\hline 0010030240 & 20.8834 \\
\hline 0010240030 & 20.8834 \\
\hline 0010030402 & 66.2014 \\
\hline 0010400032 & 66.2014 \\
\hline 0010030420 & 20.6642 \\
\hline 0010420030 & 20.6642 \\
\hline 0010032004 & 49.8100 \\
\hline 0012000034 & 49.8100 \\
\hline 0010032040 & 13.5286 \\
\hline 0012040030 & 13.5286 \\
\hline 0010032400 & $33.174 \subseteq$ \\
\hline 0012400030 & 33.1749 \\
\hline 0010034002 & 70.8113 \\
\hline 0014000032 & 70.8113 \\
\hline 0010034020 & 17.3935 \\
\hline 0014020030 & 17.3935 \\
\hline 0010034200 & 17.8264 \\
\hline 0014200030 & 17.826 \\
\hline 0010040203 & 39.4173 \\
\hline 0010200043 & 39.4173 \\
\hline 0010040230 & 21.2035 \\
\hline 0010230040 & 21.2035 \\
\hline 0010040302 & 62.982 \\
\hline 0010300042 & 62.982 \\
\hline 0010040320 & 11.2398 \\
\hline 0010320040 & 11.2398 \\
\hline 0010042003 & 53.8837 \\
\hline 0012000043 & 53.8837 \\
\hline 0010042030 & 13.522 \\
\hline 0012030040 & 13.522 \\
\hline 0010042300 & 25.125 \\
\hline 0012300040 & 25.125 \\
\hline 0010043002 & 71.794 \\
\hline 0013000042 & 71.794 \\
\hline 0010043020 & 1.0010 \\
\hline 0013020040 & 1.0010 \\
\hline 0010043200 & 32.855 \\
\hline 0013200040 & 32.855 \\
\hline 0010200304 & 27.953 \\
\hline 0010300204 & 27.953 \\
\hline 0010200340 & 19.274 \\
\hline 0010340200 & 19.274 \\
\hline 0010200403 & 33.162 \\
\hline 0010400203 & 33.162 \\
\hline 0010200430 & 24.254 \\
\hline
\end{tabular}




\begin{tabular}{|c|c|}
\hline 0010430200 & 24.2549 \\
\hline 0010203004 & 35.0265 \\
\hline 0013000204 & 35.0265 \\
\hline 0010203040 & 5.9418 \\
\hline 0013040200 & 5.9418 \\
\hline 0010203400 & 17.0166 \\
\hline 0013400200 & 17.0166 \\
\hline 0010204003 & 34.9146 \\
\hline 0014000203 & 34.9146 \\
\hline 0010204030 & 8.0317 \\
\hline 0014030200 & 8.0317 \\
\hline 0010204300 & 8.4767 \\
\hline 0014300200 & 8.4767 \\
\hline 0010230400 & 26.7926 \\
\hline 0010400230 & 26.7926 \\
\hline 0010234000 & 41.1383 \\
\hline 0014000230 & 41.1383 \\
\hline 0010240300 & 36.8108 \\
\hline 0010300240 & 36.8108 \\
\hline 0010243000 & 35.7905 \\
\hline 0013000240 & 35.7905 \\
\hline 0010300402 & 56.5 \\
\hline 0010400302 & 56.55 \\
\hline 0010300420 & 27.22 \\
\hline 0010420300 & 27.2213 \\
\hline 0010302004 & 41.2912 \\
\hline 0012000304 & 41.2912 \\
\hline 0010302040 & 13.463 \\
\hline 0012040300 & 13.463 \\
\hline 0010302400 & 33.49 \\
\hline 0012400300 & 33.4945 \\
\hline 0010304002 & 66.1918 \\
\hline 0014000302 & 66.1918 \\
\hline 0010304020 & 6.4103 \\
\hline 0014020300 & 6.4103 \\
\hline 0010304200 & 14.620 \\
\hline 0014200300 & 14.620 \\
\hline 0010320400 & $17.421<$ \\
\hline 0010400320 & 17.421 \\
\hline 0010324000 & 26.596 \\
\hline 0014000320 & 26.596 \\
\hline 0010342000 & $32.450 \leqq$ \\
\hline 0012000340 & 32.450 \\
\hline 0010402003 & 51.4157 \\
\hline 0012000403 & 51.415 \\
\hline 0010402030 & 19.228 \\
\hline 0012030400 & 19.228 \\
\hline 0010402300 & 31.604 \\
\hline 0012300400 & 31.604 \\
\hline 00104 & 62.371 \\
\hline
\end{tabular}




\begin{tabular}{|c|c|}
\hline 0013000402 & 62.3716 \\
\hline 0010403020 & 2.8709 \\
\hline 0013020400 & 2.8709 \\
\hline 0010403200 & 35.6063 \\
\hline 0013200400 & 35.6063 \\
\hline 0010423000 & 26.8616 \\
\hline 0013000420 & 26.8616 \\
\hline 0010432000 & 37.9313 \\
\hline 0012000430 & 37.9313 \\
\hline 0012003004 & 67.4685 \\
\hline 0013002004 & 67.4685 \\
\hline 0012003040 & 35.8997 \\
\hline 0013042000 & 35.8997 \\
\hline 0012003400 & 74.1115 \\
\hline 0013402000 & 74.1115 \\
\hline 0012004003 & 66.0731 \\
\hline 0014002003 & 66.0731 \\
\hline 0012004030 & 35.2262 \\
\hline 0014032000 & 35.2262 \\
\hline 0012004300 & 39.6877 \\
\hline 0014302000 & 39.6877 \\
\hline 0012034000 & 37.1683 \\
\hline 0014002030 & 37.1683 \\
\hline 0012043000 & 34.4605 \\
\hline 0013002040 & 34.4605 \\
\hline 0012304000 & 46.0884 \\
\hline 0014002300 & 46.0884 \\
\hline 0012403000 & 59.6781 \\
\hline 0013002400 & 59.6781 \\
\hline 0013004002 & 68.8864 \\
\hline 0014003002 & 68.8864 \\
\hline 0013004020 & 11.6220 \\
\hline 0014023000 & 11.6220 \\
\hline 0013004200 & 46.3486 \\
\hline 0014203000 & 46.3486 \\
\hline 0013024000 & 9.6794 \\
\hline 0014003020 & 9.6794 \\
\hline 0013204000 & 45.0729 \\
\hline 0014003200 & 45.0729 \\
\hline 0020030041 & 122.1043 \\
\hline 0020040031 & 122.1043 \\
\hline 0020030104 & 44.2888 \\
\hline 0020100034 & 44.2888 \\
\hline 0020030140 & 16.3801 \\
\hline 0020140030 & 16.3801 \\
\hline 0020030401 & 81.4905 \\
\hline 0020400031 & 81.4905 \\
\hline 0020030410 & 15.9139 \\
\hline 0020410030 & 15.9139 \\
\hline 0020031004 & 47.5321 \\
\hline
\end{tabular}




\begin{tabular}{|c|c|}
\hline 0021000034 & 47.5321 \\
\hline 0020031040 & 21.1058 \\
\hline 0021040030 & 21.1058 \\
\hline 0020031400 & 38.2002 \\
\hline 0021400030 & 38.2002 \\
\hline 0020034001 & 83.9854 \\
\hline 0024000031 & 83.9854 \\
\hline 0020034010 & 0.8602 \\
\hline 0024010030 & 0.8602 \\
\hline 0020034100 & 33.5358 \\
\hline 0024100030 & 33.5358 \\
\hline 0020040103 & 49.524 \\
\hline 0020100043 & 49.524 \\
\hline 0020040130 & 14.5372 \\
\hline 0020130040 & 14.5372 \\
\hline 0020040301 & 75.6357 \\
\hline 0020300041 & 75.6357 \\
\hline 0020040310 & 18.6526 \\
\hline 0020310040 & 18.652 \\
\hline 0020041003 & 53.490 \\
\hline 0021000043 & 53.4903 \\
\hline 0020041030 & 20.846 \\
\hline 0021030040 & 20.8463 \\
\hline 0020041300 & 30.452 \\
\hline 0021300040 & 30.452 \\
\hline 0020043001 & 83.8555 \\
\hline 0023000041 & 83.8555 \\
\hline 0020043010 & 1.6379 \\
\hline 0023010040 & 1.6379 \\
\hline 0020043100 & 28.785 \\
\hline 0023100040 & 28.785 \\
\hline 0020100304 & 34.683 \\
\hline 0020300104 & 34.683 \\
\hline 0020100340 & 18.045 \\
\hline 0020340100 & 18.045 \\
\hline 0020100403 & 44.4106 \\
\hline 0020400103 & 44.410 \\
\hline 0020100430 & 17.4684 \\
\hline 0020430100 & 17.468 \\
\hline 0020103004 & $46.274 \varsigma$ \\
\hline 0023000104 & 46.274 \\
\hline 0020103040 & 32.490 \\
\hline 0023040100 & 32.490 \\
\hline 0020103400 & 18.341 \\
\hline 0023400100 & 18.341 \\
\hline 0020104003 & 47.196 \\
\hline 0024000103 & 47.196 \\
\hline 0020104030 & 9.3050 \\
\hline 0024030100 & 9.3050 \\
\hline 0020104300 & 9.0903 \\
\hline
\end{tabular}




\begin{tabular}{|c|c|}
\hline 0024300100 & 9.0903 \\
\hline 0020130400 & 25.3051 \\
\hline 0020400130 & 25.3051 \\
\hline 0020134000 & 25.9913 \\
\hline 0024000130 & 25.9913 \\
\hline 0020140300 & 17.8470 \\
\hline 0020300140 & 17.8470 \\
\hline 0020143000 & 20.9750 \\
\hline 0023000140 & 20.9750 \\
\hline 0020300401 & 50.7367 \\
\hline 0020400301 & 50.7367 \\
\hline 0020300410 & 12.4076 \\
\hline 0020410300 & 12.4076 \\
\hline 0020301004 & 47.134 \\
\hline 0021000304 & 47.1340 \\
\hline 0020301040 & 2.8512 \\
\hline 0021040300 & 2.8512 \\
\hline 0020301400 & 30.1526 \\
\hline 0021400300 & 30.1526 \\
\hline 0020304001 & 58.6087 \\
\hline 0024000301 & 58.608 \\
\hline 0020304010 & 4.3728 \\
\hline 0024010300 & 4.3728 \\
\hline 0020304100 & $29.807 \varepsilon$ \\
\hline 0024100300 & 29.807 \\
\hline 0020310400 & 24.521 \\
\hline 0020400310 & 24.5215 \\
\hline 0020314000 & 25.135 \\
\hline 0024000310 & 25.135 \\
\hline 0020341000 & 18.3630 \\
\hline 0021000340 & 18.363 \\
\hline 0020401003 & 46.270 \\
\hline 0021000403 & 46.270 \\
\hline 0020401030 & 6.9821 \\
\hline 0021030400 & 6.9821 \\
\hline 0020401300 & 31.206 \\
\hline 0021300400 & 31.206 \\
\hline 0020403001 & 60.774 \\
\hline 0023000401 & 60.774 \\
\hline 0020403010 & 3.6673 \\
\hline 0023010400 & 3.6673 \\
\hline 0020403100 & 29.309 \\
\hline 0023100400 & 29.309 \\
\hline 0020413000 & 21.966 \\
\hline 0023000410 & 21.966 \\
\hline 0020431000 & 26.196 \\
\hline 0021000430 & 26.196 \\
\hline 0021003004 & 70.480 \\
\hline $002300100<$ & 70.480 \\
\hline 002100304 & 31.683 \\
\hline
\end{tabular}




\begin{tabular}{|c|c|}
\hline 0023041000 & 31.6838 \\
\hline 0021003400 & 41.4653 \\
\hline 0023401000 & 41.4653 \\
\hline 0021004003 & 66.1538 \\
\hline 0024001003 & 66.1538 \\
\hline 0021004030 & 34.0494 \\
\hline 0024031000 & 34.0494 \\
\hline 0021004300 & 34.6647 \\
\hline 0024301000 & 34.6647 \\
\hline 0021034000 & 27.8287 \\
\hline 0024001030 & 27.8287 \\
\hline 0021043000 & 32.0714 \\
\hline 0023001040 & 32.0714 \\
\hline 0021304000 & 65.7019 \\
\hline 0024001300 & 65.7019 \\
\hline 0021403000 & 65.2236 \\
\hline 0023001400 & 65.2236 \\
\hline 0023004001 & 63.3975 \\
\hline 0024003001 & 63.3975 \\
\hline 0023004010 & 10.3703 \\
\hline 0024013000 & 10.3703 \\
\hline 0023004100 & 55.4210 \\
\hline 0024103000 & 55.4210 \\
\hline 0023014000 & 6.7775 \\
\hline 0024003010 & 6.7775 \\
\hline 0023104000 & 55.4462 \\
\hline 0024003100 & 55.4462 \\
\hline 0030040102 & 71.4672 \\
\hline 0030100042 & 71.4672 \\
\hline 0030040120 & 26.5889 \\
\hline 0030120040 & 26.5889 \\
\hline 0030040201 & 70.1718 \\
\hline 0030200041 & 70.1718 \\
\hline 0030040210 & 25.8102 \\
\hline 0030210040 & 25.8102 \\
\hline 0030041002 & 75.8886 \\
\hline 0031000042 & 75.8886 \\
\hline 0030041020 & 21.6958 \\
\hline 0031020040 & 21.6958 \\
\hline 0030041200 & 48.4342 \\
\hline 0031200040 & 48.4342 \\
\hline 0030042001 & 85.8295 \\
\hline 0032000041 & 85.8295 \\
\hline 0030042010 & 13.2916 \\
\hline 0032010040 & 13.2916 \\
\hline 0030042100 & 36.8247 \\
\hline 0032100040 & 36.8247 \\
\hline 0030100204 & 16.9418 \\
\hline 0030200104 & 16.9418 \\
\hline 0030100240 & 32.1323 \\
\hline
\end{tabular}




\begin{tabular}{|c|c|}
\hline 0030240100 & 32.1323 \\
\hline 0030100402 & 54.5749 \\
\hline 0030400102 & 54.5749 \\
\hline 0030100420 & 26.0391 \\
\hline 0030420100 & 26.035 \\
\hline 0030102004 & 26.720 \\
\hline 0032000104 & 26.720 \\
\hline 0030102040 & 22.325 \\
\hline 0032040100 & 22.325 \\
\hline 0030102400 & 43.147 \\
\hline 0032400100 & 43.147 \\
\hline 0030104002 & 62.33 \\
\hline 0034000102 & 62.33 \\
\hline 0030104020 & 14.297 \\
\hline 0034020100 & 14.297 \\
\hline 0030104200 & 29.192 \\
\hline 0034200100 & 29.192 \\
\hline 0030120400 & 30.928 \\
\hline 0030400120 & 30.928 \\
\hline 0030124000 & 34.710 \\
\hline 0034000120 & 34.710 \\
\hline 0030140200 & 25.656 \\
\hline 0030200140 & 25.656 \\
\hline 0030142000 & $36.21 \varepsilon$ \\
\hline 0032000140 & 36.21 \\
\hline 0030200401 & 55.776 \\
\hline 0030400201 & 55.776 \\
\hline 0030200410 & 22.413 \\
\hline 0030410200 & 22.41 \\
\hline 0030201004 & 20.3632 \\
\hline 0031000204 & 20.363 \\
\hline 0030201040 & 14.683 \\
\hline 0031040200 & 14.6836 \\
\hline 0030201400 & 42.2840 \\
\hline 0031400200 & 42.284 \\
\hline 0030204001 & 53.068 \\
\hline 0034000201 & 53.0687 \\
\hline 0030204010 & 9.7338 \\
\hline 0034010200 & 9.7338 \\
\hline 0030204100 & $37.766 c$ \\
\hline 0034100200 & 37.766 \\
\hline 0030210400 & 32.505 \\
\hline 0030400210 & 32.505 \\
\hline 0030214000 & 34.086 \\
\hline 0034000210 & 34.086 \\
\hline 0030241000 & 36.848 \\
\hline 0031000240 & 36.848 \\
\hline 0030401002 & 65.290 \\
\hline 0031000402 & 65.2908 \\
\hline 0030401020 & 17.134 \\
\hline
\end{tabular}




\begin{tabular}{|c|c|}
\hline 0031020400 & 17.1347 \\
\hline 0030401200 & 51.7026 \\
\hline 0031200400 & 51.7026 \\
\hline 0030402001 & 63.7705 \\
\hline 0032000401 & 63.7705 \\
\hline 0030402010 & 18.3698 \\
\hline 0032010400 & 18.3698 \\
\hline 0030402100 & 45.2572 \\
\hline 0032100400 & 45.2572 \\
\hline 0030412000 & 61.5897 \\
\hline 0032000410 & 61.5897 \\
\hline 0030421000 & 40.0189 \\
\hline 0031000420 & 40.0189 \\
\hline 0031002004 & 54.7718 \\
\hline 0032001004 & 54.7718 \\
\hline 0031002040 & 46.2355 \\
\hline 0032041000 & 46.2355 \\
\hline 0031002400 & 78.4316 \\
\hline 0032401000 & 78.4316 \\
\hline 0031004002 & 85.0583 \\
\hline 0034001002 & 85.0583 \\
\hline 0031004020 & 40.9558 \\
\hline 0034021000 & 40.9558 \\
\hline 0031004200 & 49.7184 \\
\hline 0034201000 & 49.7184 \\
\hline 0031024000 & 34.4122 \\
\hline 0034001020 & 34.4122 \\
\hline 0031042000 & 45.5447 \\
\hline 0032001040 & 45.5447 \\
\hline 0031204000 & 74.5951 \\
\hline 0034001200 & 74.5951 \\
\hline 0031402000 & 81.8087 \\
\hline 0032001400 & 81.8087 \\
\hline 0032004001 & 79.9955 \\
\hline 0034002001 & 79.9955 \\
\hline 0032004010 & 36.7471 \\
\hline 0034012000 & 36.7471 \\
\hline 0032004100 & 76.2604 \\
\hline 0034102000 & 76.2604 \\
\hline 0032014000 & 36.9965 \\
\hline 0034002010 & 36.9965 \\
\hline 0032104000 & 59.8837 \\
\hline 0034002100 & 59.8837 \\
\hline 0040100203 & 26.0424 \\
\hline 0040200103 & 26.0424 \\
\hline 0040100230 & 26.1655 \\
\hline 0040230100 & 26.1655 \\
\hline 0040100302 & 54.8403 \\
\hline 0040300102 & 54.8403 \\
\hline 0040100320 & 20.2808 \\
\hline
\end{tabular}




\begin{tabular}{|c|c|}
\hline 0040320100 & 20.2808 \\
\hline 0040102003 & 37.4780 \\
\hline 0042000103 & 37.4780 \\
\hline 0040102030 & 17.6546 \\
\hline 0042030100 & 17.6546 \\
\hline 0040102300 & 33.1653 \\
\hline 0042300100 & 33.1653 \\
\hline 0040103002 & 58.8846 \\
\hline 0043000102 & 58.8846 \\
\hline 0040103020 & 4.5988 \\
\hline 0043020100 & 4.5988 \\
\hline 0040103200 & 36.5014 \\
\hline 0043200100 & 36.501 \\
\hline 0040120300 & 18.640 \\
\hline 0040300120 & 18.64 \\
\hline 0040123000 & 24.656 \\
\hline 0043000120 & 24.656 \\
\hline 0040130200 & 26.908 \\
\hline 0040200130 & $26.908 \Im$ \\
\hline 0040132000 & 37.364 \\
\hline 0042000130 & 37.364 \\
\hline 0040200301 & $49.800 \varepsilon$ \\
\hline 0040300201 & 49.8008 \\
\hline 0040200310 & 22.302 \\
\hline 0040310200 & 22.302 \\
\hline 0040201003 & 29.776 \\
\hline 0041000203 & 29.776 \\
\hline 0040201030 & 7.9146 \\
\hline 0041030200 & 7.9146 \\
\hline 0040201300 & 35.840 \\
\hline 0041300200 & 35.840 \\
\hline 0040203001 & 53.982 \\
\hline 0043000201 & 53.982 \\
\hline 0040203010 & 10.701 \\
\hline 0043010200 & 10.7017 \\
\hline 0040203100 & 34.323 \\
\hline 0043100200 & 34.323 \\
\hline 0040210300 & 22.259 \\
\hline 0040300210 & 22.259 \\
\hline 0040213000 & 26.958 \\
\hline 0043000210 & 26.958 \\
\hline 0040231000 & 36.104 \\
\hline 0041000230 & 36.104 \\
\hline 0040301002 & 58.020 \\
\hline 0041000302 & 58.020 \\
\hline 0040301020 & 4.4461 \\
\hline 0041020300 & 4.4461 \\
\hline 0040301200 & 41.463 \\
\hline 0041200300 & 41.463 \\
\hline 0040302001 & 62.904 \\
\hline
\end{tabular}




\begin{tabular}{|c|c|}
\hline 0042000301 & 62.9048 \\
\hline 0040302010 & 12.5223 \\
\hline 0042010300 & 12.5223 \\
\hline 0040302100 & 41.3066 \\
\hline 0042100300 & 41.3066 \\
\hline 0040312000 & $35.014 C$ \\
\hline 0042000310 & $35.014 C$ \\
\hline 0040321000 & 22.4547 \\
\hline 0041000320 & 22.4547 \\
\hline 0041002003 & 60.385 \\
\hline 0042001003 & 60.385 \\
\hline 0041002030 & 39.70 \\
\hline 0042031000 & 39.70 \\
\hline 0041002300 & $58.2 s$ \\
\hline 0042301000 & 58.20 \\
\hline 0041003002 & 84.17 \\
\hline 0043001002 & 84.17 \\
\hline 0041003020 & 32.40 \\
\hline 0043021000 & 32.405 \\
\hline 0041003200 & 64.2346 \\
\hline 0043201000 & 64.234 \\
\hline 0041023000 & 32.89 \\
\hline 0043001020 & 32.894 \\
\hline 0041032000 & 38.699 \\
\hline 0042001030 & 38.699 \\
\hline 0041203000 & 62.238 \\
\hline 0043001200 & 62.238 \\
\hline 0041302000 & 78.410 \\
\hline 0042001300 & 78.410 \\
\hline 0042003001 & 81.764 \\
\hline 0043002001 & 81.7645 \\
\hline 0042003010 & 33.620 \\
\hline 0043012000 & 33.620 \\
\hline 0042003100 & 62.673 \\
\hline 0043102000 & 62.673 \\
\hline 0042013000 & 33.270 \\
\hline 0043002010 & 33.270 \\
\hline 0042103000 & 58.627 \\
\hline 0043002100 & 58.627 \\
\hline 0100200304 & 19.808 \\
\hline 0100300204 & 19.808 \\
\hline 0100200340 & 23.596 \\
\hline 0100340200 & 23.596 \\
\hline 0100200403 & 24.022 \\
\hline 0100400203 & 24.022 \\
\hline 0100200430 & 26.623 \\
\hline 0100430200 & 26.623 \\
\hline 0100203004 & 27.126 \\
\hline 0103000204 & 27.126 \\
\hline 0100203 & 17.010 \\
\hline
\end{tabular}




\begin{tabular}{|c|c|}
\hline 0103040200 & 17.0101 \\
\hline 0100203400 & 21.6135 \\
\hline 0103400200 & 21.6135 \\
\hline 0100204003 & 20.4736 \\
\hline 0104000203 & 20.473 \\
\hline 0100204030 & 16.473 \\
\hline 0104030200 & 16.473 \\
\hline 0100204300 & 15.7642 \\
\hline 0104300200 & 15.7642 \\
\hline 0100230400 & 32.4826 \\
\hline 0100400230 & 32.482 \\
\hline 0100234000 & 33.3672 \\
\hline 0104000230 & 33.3672 \\
\hline 0100240300 & 35.8543 \\
\hline 0100300240 & 35.854 \\
\hline 0100243000 & 38.255 \\
\hline 0103000240 & 38.255 \\
\hline 0100300402 & 48.053 \\
\hline 0100400302 & 48.0532 \\
\hline 0100300420 & 25.312 \\
\hline 0100420300 & 25.3129 \\
\hline 0100302004 & 30.5971 \\
\hline 0102000304 & 30.597 \\
\hline 0100302040 & 21.050 \\
\hline 0102040300 & 21.0505 \\
\hline 0100302400 & 36.9532 \\
\hline 0102400300 & 36.9532 \\
\hline 0100304002 & 57.115 \\
\hline 0104000302 & 57.115 \\
\hline 0100304020 & 11.710 \\
\hline 0104020300 & 11.710 \\
\hline 0100304200 & 22.816 \\
\hline 0104200300 & 22.816 \\
\hline 0100320400 & 23.6327 \\
\hline 0100400320 & 23.6327 \\
\hline 0100324000 & 31.0225 \\
\hline 0104000320 & 31.022 \\
\hline 0100342000 & 35.035 \\
\hline 0102000340 & 35.0353 \\
\hline 0100402003 & 35.2622 \\
\hline 0102000403 & 35.262 \\
\hline 0100402030 & 27.083 \\
\hline 0102030400 & 27.083 \\
\hline 0100402300 & 35.422 \\
\hline 0102300400 & 35.422 \\
\hline 0100403002 & 53.217 \\
\hline 0103000402 & 53.2170 \\
\hline 0100403020 & 7.8966 \\
\hline 0103020400 & 7.8966 \\
\hline 0100403200 & 40.656 \\
\hline
\end{tabular}




\begin{tabular}{|c|c|}
\hline 0103200400 & 40.6561 \\
\hline 0100423000 & 29.9297 \\
\hline 0103000420 & 29.9297 \\
\hline 0100432000 & 37.0475 \\
\hline 0102000430 & 37.0475 \\
\hline 0102003004 & 49.4000 \\
\hline 0103002004 & 49.4000 \\
\hline 0102003040 & 39.2511 \\
\hline 0103042000 & 39.251 \\
\hline 0102003400 & 48.702 \\
\hline 0103402000 & 48.702 \\
\hline 0102004003 & 48.077 \\
\hline 0104002003 & 48.0778 \\
\hline 0102004030 & 40.5256 \\
\hline 0104032000 & 40.5256 \\
\hline 0102004300 & 43.255 \\
\hline 0104302000 & $43.255 \subseteq$ \\
\hline 0102034000 & 45.8243 \\
\hline 0104002030 & 45.824 \\
\hline 0102043000 & 37.7546 \\
\hline 0103002040 & 37.7546 \\
\hline 0102304000 & 56.598 \\
\hline 0104002300 & 56.5988 \\
\hline 0102403000 & 61.0926 \\
\hline 0103002400 & 61.092 \\
\hline 0103004002 & 61.0732 \\
\hline 0104003002 & 61.0732 \\
\hline 0103004020 & 13.8108 \\
\hline 0104023000 & 13.8108 \\
\hline 0103004200 & 50.7773 \\
\hline 0104203000 & 50.7773 \\
\hline 0103024000 & 14.110 \\
\hline 0104003020 & 14.1106 \\
\hline 0103204000 & $47.980 \Im$ \\
\hline 0104003200 & 47.980 \\
\hline 0120300400 & 26.203 \\
\hline 0120400300 & 26.203 \\
\hline 0120304000 & 36.844 \\
\hline 0124000300 & 36.8447 \\
\hline 0120403000 & 31.956 \\
\hline 0123000400 & 31.9561 \\
\hline 0123004000 & 38.269 \\
\hline 0124003000 & 38.2692 \\
\hline 0130200400 & 32.279 \\
\hline 0130400200 & 32.2792 \\
\hline 0130204000 & 37.0237 \\
\hline 0134000200 & 37.023 \\
\hline 0130402000 & 39.503 \\
\hline 0132000400 & 39.5038 \\
\hline 0132004000 & 58.590 \\
\hline
\end{tabular}




\begin{tabular}{|c|c|}
\hline 0134002000 & 58.5904 \\
\hline 0140200300 & 26.0275 \\
\hline 0140300200 & 26.0275 \\
\hline 0140203000 & 27.2966 \\
\hline 0143000200 & 27.2966 \\
\hline 0140302000 & 35.4287 \\
\hline 0142000300 & 35.4287 \\
\hline 0142003000 & 56.2498 \\
\hline 0143002000 & 56.249 \\
\hline 0200300401 & 27.130 \\
\hline 0200400301 & 27.130 \\
\hline 0200300410 & 21.880 \\
\hline 0200410300 & 21.8802 \\
\hline 0200301004 & 23.2493 \\
\hline 0201000304 & 23.249 \\
\hline 0200301040 & 9.9436 \\
\hline 0201040300 & 9.9436 \\
\hline 0200301400 & 37.719 \\
\hline 0201400300 & $37.71 \mathrm{c}$ \\
\hline 0200304001 & 36.2785 \\
\hline 0204000301 & 36.27 \\
\hline 0200304010 & 10.776 \\
\hline 0204010300 & 10.776 \\
\hline 0200304100 & 38.027 \\
\hline 0204100300 & 38.027 \\
\hline 0200310400 & 31.720 \\
\hline 0200400310 & 31.7200 \\
\hline 0200314000 & 31.603 \\
\hline 0204000310 & 31.603 \\
\hline 0200341000 & 24.916 \\
\hline 0201000340 & 24.916 \\
\hline 0200401003 & 25.065 \\
\hline 0201000403 & 25.0656 \\
\hline 0200401030 & 17.421 \\
\hline 0201030400 & 17.421 \\
\hline 0200401300 & 37.068 \\
\hline 0201300400 & 37.068 \\
\hline 0200403001 & 34.282 \\
\hline 0203000401 & 34.282 \\
\hline 0200403010 & 8.6822 \\
\hline 0203010400 & 8.6822 \\
\hline 0200403100 & 39.225 \\
\hline 0203100400 & 39.225 \\
\hline 0200413000 & 29.742 \\
\hline 0203000410 & 29.742 \\
\hline 0200431000 & 60.090 \\
\hline 0201000430 & 60.090 \\
\hline 0201003004 & 49.636 \\
\hline 0203001004 & 49.636 \\
\hline 0201003040 & 36.693 \\
\hline
\end{tabular}




\begin{tabular}{|c|c|}
\hline 0203041000 & 36.6939 \\
\hline 0201003400 & 47.1476 \\
\hline 0203401000 & 47.1476 \\
\hline 0201004003 & 45.3202 \\
\hline 0204001003 & 45.3202 \\
\hline 0201004030 & $36.829 \varsigma$ \\
\hline 0204031000 & 36.8299 \\
\hline 0201004300 & 43.6978 \\
\hline 0204301000 & 43.6978 \\
\hline 0201034000 & 33.4910 \\
\hline 0204001030 & 33.4910 \\
\hline 0201043000 & 38.439 \\
\hline 0203001040 & 38.439 \\
\hline 0201304000 & 74.134 \\
\hline 0204001300 & 74.1340 \\
\hline 0201403000 & $69.627 \varepsilon$ \\
\hline 0203001400 & $69.627 \varepsilon$ \\
\hline 0203004001 & 38.9289 \\
\hline 0204003001 & $38.928 \subseteq$ \\
\hline 0203004010 & 14.9818 \\
\hline 0204013000 & $14.981 \varepsilon$ \\
\hline 0203004100 & 62.0480 \\
\hline 0204103000 & 62.0480 \\
\hline 0203014000 & 12.9286 \\
\hline 0204003010 & 12.928 \\
\hline 0203104000 & 53.748 \\
\hline 0204003100 & 53.748 \\
\hline 0210300400 & 30.284 \\
\hline 0210400300 & 30.284 \\
\hline 0210304000 & $39.827<$ \\
\hline 0214000300 & 39.8274 \\
\hline 0210403000 & 35.7217 \\
\hline 0213000400 & 35.7217 \\
\hline 0213004000 & 40.238 \\
\hline 0214003000 & 40.238 \\
\hline 0230401000 & 39.597 \\
\hline 0231000400 & 39.5977 \\
\hline 0231004000 & 57.242 \\
\hline 0234001000 & 57.242 \\
\hline 0240301000 & 38.049 \\
\hline 0241000300 & 38.049 \\
\hline 0241003000 & 62.7837 \\
\hline 0243001000 & 62.7837 \\
\hline 0300401002 & 50.7485 \\
\hline 0301000402 & 50.7485 \\
\hline 0300401020 & 5.8032 \\
\hline 0301020400 & 5.8032 \\
\hline 0300401200 & 45.2997 \\
\hline 0301200400 & 45.2997 \\
\hline 0300402001 & 39.0727 \\
\hline
\end{tabular}




\begin{tabular}{|c|c|}
\hline 0302000401 & 39.0727 \\
\hline 0300402010 & 15.3755 \\
\hline 0302010400 & 15.3755 \\
\hline 0300402100 & 41.5450 \\
\hline 0302100400 & 41.5450 \\
\hline 0300412000 & 33.6023 \\
\hline 0302000410 & 33.6023 \\
\hline 0300421000 & 34.650 \\
\hline 0301000420 & 34.6506 \\
\hline 0301002004 & 50.0105 \\
\hline 0302001004 & 50.010 \\
\hline 0301002040 & 38.5712 \\
\hline 0302041000 & 38.5712 \\
\hline 0301002400 & 61.113 \\
\hline 0302401000 & 61.113 \\
\hline 0301004002 & 76.019 \\
\hline 0304001002 & 76.019 \\
\hline 0301004020 & 30.2052 \\
\hline 0304021000 & 30.2052 \\
\hline 0301004200 & 47.4272 \\
\hline 0304201000 & 47.427 \\
\hline 0301024000 & 31.917 \\
\hline 0304001020 & 31.917 \\
\hline 0301042000 & 41.715 \\
\hline 0302001040 & 41.715 \\
\hline 0301204000 & 63.656 \\
\hline 0304001200 & 63.656 \\
\hline 0301402000 & 79.760 \\
\hline 0302001400 & 79.760 \\
\hline 0302004001 & 57.4118 \\
\hline 0304002001 & 57.4118 \\
\hline 0302004010 & 32.464 \\
\hline 0304012000 & 32.4642 \\
\hline 0302004100 & 70.030 \\
\hline 0304102000 & 70.030 \\
\hline 0302014000 & 32.915 \\
\hline 0304002010 & 32.915 \\
\hline 0302104000 & 58.720 \\
\hline 0304002100 & 58.720 \\
\hline 0310402000 & 38.316 \\
\hline 0312000400 & 38.316 \\
\hline 0312004000 & 56.560 \\
\hline 0314002000 & 56.560 \\
\hline 0320401000 & 25.518 \\
\hline 0321000400 & 25.518 \\
\hline 0321004000 & 50.132 \\
\hline 0324001000 & 50.132 \\
\hline 0341002000 & 54.751 \\
\hline 0342001000 & 54.7513 \\
\hline 0401002003 & 56.364 \\
\hline
\end{tabular}




\begin{tabular}{|c|c|}
\hline 0402001003 & 56.3640 \\
\hline 0401002030 & 55.2492 \\
\hline 0402031000 & 55.2492 \\
\hline 0401002300 & 60.3771 \\
\hline 0402301000 & 60.3771 \\
\hline 0401003002 & 77.6176 \\
\hline 0403001002 & 77.6176 \\
\hline 0401003020 & 32.2568 \\
\hline 0403021000 & 32.2568 \\
\hline 0401003200 & 72.7924 \\
\hline 0403201000 & 72.7924 \\
\hline 0401023000 & 32.3397 \\
\hline 0403001020 & 32.3397 \\
\hline 0401032000 & 55.7767 \\
\hline 0402001030 & 55.7767 \\
\hline 0401203000 & 70.6355 \\
\hline 0403001200 & 70.6355 \\
\hline 0401302000 & 78.8531 \\
\hline 0402001300 & 78.8531 \\
\hline 0402003001 & 63.0339 \\
\hline 0403002001 & 63.0339 \\
\hline 0402003010 & 33.4200 \\
\hline 0403012000 & 33.4200 \\
\hline 0402003100 & 67.1516 \\
\hline 0403102000 & 67.1516 \\
\hline 0402013000 & 39.5670 \\
\hline 0403002010 & 39.5670 \\
\hline 0402103000 & 60.6107 \\
\hline 0403002100 & 60.6107 \\
\hline 0412003000 & 56.6386 \\
\hline 0413002000 & 56.6386 \\
\hline 0421003000 & 58.6272 \\
\hline 0423001000 & 58.6272 \\
\hline 0431002000 & 62.1132 \\
\hline 0432001000 & 62.1132 \\
\hline 1002003004 & 93.0986 \\
\hline 1003002004 & 93.0986 \\
\hline 1002003040 & 96.6598 \\
\hline 1003042000 & 96.6598 \\
\hline 1002003400 & 95.3641 \\
\hline 1003402000 & 95.3641 \\
\hline 1002004003 & 105.9880 \\
\hline 1004002003 & 105.9880 \\
\hline 1002004030 & 84.0666 \\
\hline 1004032000 & 84.0666 \\
\hline 1002004300 & 105.3279 \\
\hline 1004302000 & 105.3279 \\
\hline 1002034000 & 85.0679 \\
\hline 1004002030 & 85.0679 \\
\hline 1002043000 & 82.4075 \\
\hline
\end{tabular}




$\begin{array}{ll}1003002040 & 82.4075 \\ 1002304000 & 105.8221 \\ 1004002300 & 105.8221 \\ 1002403000 & 106.3810 \\ 1003002400 & 106.3810 \\ 1003004002 & 102.4895 \\ 1004003002 & 102.4895 \\ 1003004020 & 58.8765 \\ 1004023000 & 58.8765 \\ 1003004200 & 81.3105 \\ 1004203000 & 81.3105 \\ 1003024000 & 58.9984 \\ 1004003020 & 58.9984 \\ 1003204000 & 82.9760 \\ 1004003200 & 82.9760 \\ 1023004000 & 57.0386 \\ 1024003000 & 57.0386 \\ 1032004000 & 94.5702 \\ 1034002000 & 94.5702 \\ 1042003000 & 82.2043 \\ 1043002000 & 82.2043 \\ 1203004000 & 119.3194 \\ 1204003000 & 119.3194 \\ 1302004000 & 126.0988 \\ 1304002000 & 126.0988 \\ 1402003000 & 145.9070 \\ 1403002000 & 145.9070 \\ 2003004001 & 87.0424 \\ 2004003001 & 87.0424 \\ 2003004010 & 63.8475 \\ 2004013000 & 63.8475 \\ 2003004100 & 102.1949 \\ 2004103000 & 102.1949 \\ 2003014000 & 59.6739 \\ 2004003010 & 59.6739 \\ 2003104000 & 98.9178 \\ 2004003100 & 98.9178 \\ 2013004000 & 63.6431 \\ 2014003000 & 63.6431 \\ 2103004000 & 91.0632 \\ 2104003000 & 91.0632\end{array}$

Edge list:

\begin{tabular}{lrrrr} 
isomer1 & \multicolumn{2}{c}{ isomer2 } & \multicolumn{2}{c}{ trans.state TS energy } \\
--------- & \\
0000001234 & & 0000004321 & 0000001234 \\
0000001243 & & 0000003421 & 0000001243
\end{tabular}




\begin{tabular}{|c|c|c|}
\hline 0000001324 & 0000004231 & 0000001324 \\
\hline 0000001342 & 0000002431 & 0000001342 \\
\hline 0000001423 & 0000003241 & 0000001423 \\
\hline 0000001432 & 0000002341 & 0000001432 \\
\hline 0000002134 & 0000004312 & 0000002134 \\
\hline 0000002143 & 0000003412 & 0000002143 \\
\hline 0000002314 & 0000004132 & 0000002314 \\
\hline 0000002413 & 0000003142 & 0000002413 \\
\hline 0000003124 & 0000004213 & 0000003124 \\
\hline 0000003214 & 0000004123 & 0000003214 \\
\hline 0000010234 & 0004321000 & 0000010234 \\
\hline 0000230014 & 0001004320 & 0010000234 \\
\hline 0000010243 & 0003421000 & 0000010243 \\
\hline 0000240013 & 0001003420 & 0010000243 \\
\hline 0000010324 & 0004231000 & 0000010324 \\
\hline 0000320014 & 0001004230 & 0010000324 \\
\hline 0000010342 & 0002431000 & 0000010342 \\
\hline 0000340012 & 0001002430 & 0010000342 \\
\hline 0000010423 & 0003241000 & 0000010423 \\
\hline 0000420013 & 0001003240 & 0010000423 \\
\hline 0000010432 & 0002341000 & 0000010432 \\
\hline 0000430012 & 0001002340 & 0010000432 \\
\hline 0000012034 & 0004301002 & 0000012034 \\
\hline 0002030014 & 0001004302 & 0010002034 \\
\hline 0000012043 & 0003401002 & 0000012043 \\
\hline 0002040013 & 0001003402 & 0010002043 \\
\hline 0000012304 & 0004031002 & 0000012304 \\
\hline 0002300014 & 0001004032 & 0010002304 \\
\hline 0000012340 & 0000431002 & 0000012340 \\
\hline 0002340010 & 0001000432 & 0010002340 \\
\hline 0000012403 & 0003041002 & 0000012403 \\
\hline 0002400013 & 0001003042 & 0010002403 \\
\hline 0000012430 & 0000341002 & 0000012430 \\
\hline 0002430010 & 0001000342 & 0010002430 \\
\hline 0000013024 & 0004201003 & 0000013024 \\
\hline 0003020014 & 0001004203 & 0010003024 \\
\hline 0000013042 & 0002401003 & 0000013042 \\
\hline 0003040012 & 0001002403 & 0010003042 \\
\hline 0000013204 & 0004021003 & 0000013204 \\
\hline 0003200014 & 0001004023 & 0010003204 \\
\hline 0000013240 & 0000421003 & 0000013240 \\
\hline 0003240010 & 0001000423 & 0010003240 \\
\hline 0000013402 & 0002041003 & 0000013402 \\
\hline 0003400012 & 0001002043 & 0010003402 \\
\hline 0000013420 & 0000241003 & 0000013420 \\
\hline 0003420010 & 0001000243 & 0010003420 \\
\hline 0000014023 & 0003201004 & 0000014023 \\
\hline 0004020013 & 0001003204 & 0010004023 \\
\hline 0000014032 & 0002301004 & 0000014032 \\
\hline 0004030012 & 0001002304 & 0010004032 \\
\hline
\end{tabular}




\begin{tabular}{|c|c|c|}
\hline 0000014203 & 0003021004 & 0000014203 \\
\hline 0004200013 & 0001003024 & 0010004203 \\
\hline 0000014230 & 0000321004 & 0000014230 \\
\hline 0004230010 & 0001000324 & 0010004230 \\
\hline 0000014302 & 0002031004 & 0000014302 \\
\hline 0004300012 & 0001002034 & 0010004302 \\
\hline 0000014320 & 0000231004 & 0000014320 \\
\hline 0004320010 & 0001000234 & 0010004320 \\
\hline 0000020134 & 0004312000 & 0000020134 \\
\hline 0000130024 & 0002004310 & 0020000134 \\
\hline 0000020143 & 0003412000 & 0000020143 \\
\hline 0000140023 & 0002003410 & 0020000143 \\
\hline 0000020314 & 0004132000 & 0000020314 \\
\hline 0000310024 & 0002004130 & 0020000314 \\
\hline 0000020341 & 0001432000 & 0000020341 \\
\hline 0000340021 & 0002001430 & 0020000341 \\
\hline 0000020413 & 0003142000 & 0000020413 \\
\hline 0000410023 & 0002003140 & 0020000413 \\
\hline 0000020431 & 0001342000 & 0000020431 \\
\hline 0000430021 & 0002001340 & 0020000431 \\
\hline 0000021034 & 0004302001 & 0000021034 \\
\hline 0001030024 & 0002004301 & 0020001034 \\
\hline 0000021043 & 0003402001 & 0000021043 \\
\hline 0001040023 & 0002003401 & 0020001043 \\
\hline 0000021304 & 0004032001 & 0000021304 \\
\hline 0001300024 & 0002004031 & 0020001304 \\
\hline 0000021340 & 0000432001 & 0000021340 \\
\hline 0001340020 & 0002000431 & 0020001340 \\
\hline 0000021403 & 0003042001 & 0000021403 \\
\hline 0001400023 & 0002003041 & 0020001403 \\
\hline 0000021430 & 0000342001 & 0000021430 \\
\hline 0001430020 & 0002000341 & 0020001430 \\
\hline 0000023014 & 0004102003 & 0000023014 \\
\hline 0003010024 & 0002004103 & 0020003014 \\
\hline 0000023041 & 0001402003 & 0000023041 \\
\hline 0003040021 & 0002001403 & 0020003041 \\
\hline 0000023104 & 0004012003 & 0000023104 \\
\hline 0003100024 & 0002004013 & 0020003104 \\
\hline 0000023140 & 0000412003 & 0000023140 \\
\hline 0003140020 & 0002000413 & 0020003140 \\
\hline 0000023401 & 0001042003 & 0000023401 \\
\hline 0003400021 & 0002001043 & 0020003401 \\
\hline 0000023410 & 0000142003 & 0000023410 \\
\hline 0003410020 & 0002000143 & 0020003410 \\
\hline 0000024013 & 0003102004 & 0000024013 \\
\hline 0004010023 & 0002003104 & 0020004013 \\
\hline 0000024031 & 0001302004 & 0000024031 \\
\hline 0004030021 & 0002001304 & 0020004031 \\
\hline 0000024103 & 0003012004 & 0000024103 \\
\hline 0004100023 & 0002003014 & 0020004103 \\
\hline
\end{tabular}




\begin{tabular}{|c|c|c|}
\hline 0000024130 & 0000312004 & 0000024130 \\
\hline 0004130020 & 0002000314 & 0020004130 \\
\hline 0000024301 & 0001032004 & 0000024301 \\
\hline 0004300021 & 0002001034 & 0020004301 \\
\hline 0000024310 & 0000132004 & 0000024310 \\
\hline 0004310020 & 0002000134 & 0020004310 \\
\hline 0000030124 & 0004213000 & 0000030124 \\
\hline 0000120034 & 0003004210 & 0030000124 \\
\hline 0000030142 & 0002413000 & 0000030142 \\
\hline 0000140032 & 0003002410 & 0030000142 \\
\hline 0000030214 & 0004123000 & 0000030214 \\
\hline 0000210034 & 0003004120 & 0030000214 \\
\hline 0000030241 & 0001423000 & 0000030241 \\
\hline 0000240031 & 0003001420 & 0030000241 \\
\hline 0000030412 & 0002143000 & 0000030412 \\
\hline 0000410032 & 0003002140 & 0030000412 \\
\hline 0000030421 & 0001243000 & 0000030421 \\
\hline 0000420031 & 0003001240 & 0030000421 \\
\hline 0000031024 & 0004203001 & 0000031024 \\
\hline 0001020034 & 0003004201 & 0030001024 \\
\hline 0000031042 & 0002403001 & 0000031042 \\
\hline 0001040032 & 0003002401 & 0030001042 \\
\hline 0000031204 & 0004023001 & 0000031204 \\
\hline 0001200034 & 0003004021 & 0030001204 \\
\hline 0000031240 & 0000423001 & 0000031240 \\
\hline 0001240030 & 0003000421 & 0030001240 \\
\hline 0000031402 & 0002043001 & 0000031402 \\
\hline 0001400032 & 0003002041 & 0030001402 \\
\hline 0000031420 & 0000243001 & 0000031420 \\
\hline 0001420030 & 0003000241 & 0030001420 \\
\hline 0000032014 & 0004103002 & 0000032014 \\
\hline 0002010034 & 0003004102 & 0030002014 \\
\hline 0000032041 & 0001403002 & 0000032041 \\
\hline 0002040031 & 0003001402 & 0030002041 \\
\hline 0000032104 & 0004013002 & 0000032104 \\
\hline 0002100034 & 0003004012 & 0030002104 \\
\hline 0000032140 & 0000413002 & 0000032140 \\
\hline 0002140030 & 0003000412 & 0030002140 \\
\hline 0000032401 & 0001043002 & 0000032401 \\
\hline 0002400031 & 0003001042 & 0030002401 \\
\hline 0000032410 & 0000143002 & 0000032410 \\
\hline 0002410030 & 0003000142 & 0030002410 \\
\hline 0000034012 & 0002103004 & 0000034012 \\
\hline 0004010032 & 0003002104 & 0030004012 \\
\hline 0000034021 & 0001203004 & 0000034021 \\
\hline 0004020031 & 0003001204 & 0030004021 \\
\hline 0000034102 & 0002013004 & 0000034102 \\
\hline 0004100032 & 0003002014 & 0030004102 \\
\hline 0000034120 & 0000213004 & 0000034120 \\
\hline 0004120030 & 0003000214 & 0030004120 \\
\hline
\end{tabular}




\begin{tabular}{|c|c|c|}
\hline 0000034201 & 0001023004 & 0000034201 \\
\hline 0004200031 & 0003001024 & 0030004201 \\
\hline 0000034210 & 0000123004 & 0000034210 \\
\hline 0004210030 & 0003000124 & 0030004210 \\
\hline 0000040123 & 0003214000 & 0000040123 \\
\hline 0000120043 & 0004003210 & 0040000123 \\
\hline 0000040132 & 0002314000 & 0000040132 \\
\hline 0000130042 & 0004002310 & 0040000132 \\
\hline 0000040213 & 0003124000 & 0000040213 \\
\hline 0000210043 & 0004003120 & 0040000213 \\
\hline 0000040231 & 0001324000 & 0000040231 \\
\hline 0000230041 & 0004001320 & 0040000231 \\
\hline 0000040312 & 0002134000 & 0000040312 \\
\hline 0000310042 & 0004002130 & 0040000312 \\
\hline 0000040321 & 0001234000 & 0000040321 \\
\hline 0000320041 & 0004001230 & 0040000321 \\
\hline 0000041023 & 0003204001 & 0000041023 \\
\hline 0001020043 & 0004003201 & 0040001023 \\
\hline 0000041032 & 0002304001 & 0000041032 \\
\hline 0001030042 & 0004002301 & 0040001032 \\
\hline 0000041203 & 0003024001 & 0000041203 \\
\hline 0001200043 & 0004003021 & 0040001203 \\
\hline 0000041230 & 0000324001 & 0000041230 \\
\hline 0001230040 & 0004000321 & 0040001230 \\
\hline 0000041302 & 0002034001 & 0000041302 \\
\hline 0001300042 & 0004002031 & 0040001302 \\
\hline 0000041320 & 0000234001 & 0000041320 \\
\hline 0001320040 & 0004000231 & 0040001320 \\
\hline 0000042013 & 0003104002 & 0000042013 \\
\hline 0002010043 & 0004003102 & 0040002013 \\
\hline 0000042031 & 0001304002 & 0000042031 \\
\hline 0002030041 & 0004001302 & 0040002031 \\
\hline 0000042103 & 0003014002 & 0000042103 \\
\hline 0002100043 & 0004003012 & 0040002103 \\
\hline 0000042130 & 0000314002 & 0000042130 \\
\hline 0002130040 & 0004000312 & 0040002130 \\
\hline 0000042301 & 0001034002 & 0000042301 \\
\hline 0002300041 & 0004001032 & 0040002301 \\
\hline 0000042310 & 0000134002 & 0000042310 \\
\hline 0002310040 & 0004000132 & 0040002310 \\
\hline 0000043012 & 0002104003 & 0000043012 \\
\hline 0003010042 & 0004002103 & 0040003012 \\
\hline 0000043021 & 0001204003 & 0000043021 \\
\hline 0003020041 & 0004001203 & 0040003021 \\
\hline 0000043102 & 0002014003 & 0000043102 \\
\hline 0003100042 & 0004002013 & 0040003102 \\
\hline 0000043120 & 0000214003 & 0000043120 \\
\hline 0003120040 & 0004000213 & 0040003120 \\
\hline 0000043201 & 0001024003 & 0000043201 \\
\hline 0003200041 & 0004001023 & 0040003201 \\
\hline
\end{tabular}




\begin{tabular}{|c|c|c|}
\hline 0000043210 & 0000124003 & 0000043210 \\
\hline 0003210040 & 0004000123 & 0040003210 \\
\hline 0000100234 & 0004320100 & 0000100234 \\
\hline 0000230104 & 0000104320 & 0100000234 \\
\hline 0000100243 & 0003420100 & 0000100243 \\
\hline 0000240103 & 0000103420 & 0100000243 \\
\hline 0000100324 & 0004230100 & 0000100324 \\
\hline 0000320104 & 0000104230 & 0100000324 \\
\hline 0000100342 & 0002430100 & 0000100342 \\
\hline 0000340102 & 0000102430 & 0100000342 \\
\hline 0000100423 & 0003240100 & 0000100423 \\
\hline 0000420103 & 0000103240 & 0100000423 \\
\hline 0000100432 & 0002340100 & 0000100432 \\
\hline 0000430102 & 0000102340 & 0100000432 \\
\hline 0000102034 & 0004300102 & 0000102034 \\
\hline 0002030104 & 0000104302 & 0100002034 \\
\hline 0000102043 & 0003400102 & 0000102043 \\
\hline 0002040103 & 0000103402 & 0100002043 \\
\hline 0000102304 & 0004030102 & 0000102304 \\
\hline 0002300104 & 0000104032 & 0100002304 \\
\hline 0000102403 & 0003040102 & 0000102403 \\
\hline 0002400103 & 0000103042 & 0100002403 \\
\hline 0000103024 & 0004200103 & 0000103024 \\
\hline 0003020104 & 0000104203 & 0100003024 \\
\hline 0000103204 & 0004020103 & 0000103204 \\
\hline 0003200104 & 0000104023 & 0100003204 \\
\hline 0000120034 & 0004302100 & 0000120034 \\
\hline 0000030124 & 0002104300 & 0120000034 \\
\hline 0000120043 & 0003402100 & 0000120043 \\
\hline 0000040123 & 0002103400 & 0120000043 \\
\hline 0000120304 & 0004032100 & 0000120304 \\
\hline 0000300124 & 0002104030 & 0120000304 \\
\hline 0000120340 & 0000432100 & 0000120340 \\
\hline 0000340120 & 0002100430 & 0120000340 \\
\hline 0000120403 & 0003042100 & 0000120403 \\
\hline 0000400123 & 0002103040 & 0120000403 \\
\hline 0000120430 & 0000342100 & 0000120430 \\
\hline 0000430120 & 0002100340 & 0120000430 \\
\hline 0000123004 & 0004002103 & 0000123004 \\
\hline 0003000124 & 0002104003 & 0120003004 \\
\hline 0000123040 & 0000402103 & 0000123040 \\
\hline 0003040120 & 0002100403 & 0120003040 \\
\hline 0000123400 & 0000042103 & 0000123400 \\
\hline 0003400120 & 0002100043 & 0120003400 \\
\hline 0000124003 & 0003002104 & 0000124003 \\
\hline 0004000123 & 0002103004 & 0120004003 \\
\hline 0000124030 & 0000302104 & 0000124030 \\
\hline 0004030120 & 0002100304 & 0120004030 \\
\hline 0000124300 & 0000032104 & 0000124300 \\
\hline 0004300120 & 0002100034 & 0120004300 \\
\hline
\end{tabular}




\begin{tabular}{|c|c|c|}
\hline 0000130024 & 0004203100 & 0000130024 \\
\hline 0000020134 & 0003104200 & 0130000024 \\
\hline 0000130042 & 0002403100 & 0000130042 \\
\hline 0000040132 & 0003102400 & 0130000042 \\
\hline 0000130204 & 0004023100 & 0000130204 \\
\hline 0000200134 & 0003104020 & 0130000204 \\
\hline 0000130240 & 0000423100 & 0000130240 \\
\hline 0000240130 & 0003100420 & 0130000240 \\
\hline 0000130402 & 0002043100 & 0000130402 \\
\hline 0000400132 & 0003102040 & 0130000402 \\
\hline 0000130420 & 0000243100 & 0000130420 \\
\hline 0000420130 & 0003100240 & 0130000420 \\
\hline 0000132004 & 0004003102 & 0000132004 \\
\hline 0002000134 & 0003104002 & 0130002004 \\
\hline 0000132040 & 0000403102 & 0000132040 \\
\hline 0002040130 & 0003100402 & 0130002040 \\
\hline 0000132400 & 0000043102 & 0000132400 \\
\hline 0002400130 & 0003100042 & 0130002400 \\
\hline 0000134002 & 0002003104 & 0000134002 \\
\hline 0004000132 & 0003102004 & 0130004002 \\
\hline 0000134020 & 0000203104 & 0000134020 \\
\hline 0004020130 & 0003100204 & 0130004020 \\
\hline 0000134200 & 0000023104 & 0000134200 \\
\hline 0004200130 & 0003100024 & 0130004200 \\
\hline 0000140023 & 0003204100 & 0000140023 \\
\hline 0000020143 & 0004103200 & 0140000023 \\
\hline 0000140032 & 0002304100 & 0000140032 \\
\hline 0000030142 & 0004102300 & 0140000032 \\
\hline 0000140203 & 0003024100 & 0000140203 \\
\hline 0000200143 & 0004103020 & 0140000203 \\
\hline 0000140230 & 0000324100 & 0000140230 \\
\hline 0000230140 & 0004100320 & 0140000230 \\
\hline 0000140302 & 0002034100 & 0000140302 \\
\hline 0000300142 & 0004102030 & 0140000302 \\
\hline 0000140320 & 0000234100 & 0000140320 \\
\hline 0000320140 & 0004100230 & 0140000320 \\
\hline 0000142003 & 0003004102 & 0000142003 \\
\hline 0002000143 & 0004103002 & 0140002003 \\
\hline 0000142030 & 0000304102 & 0000142030 \\
\hline 0002030140 & 0004100302 & 0140002030 \\
\hline 0000142300 & 0000034102 & 0000142300 \\
\hline 0002300140 & 0004100032 & 0140002300 \\
\hline 0000143002 & 0002004103 & 0000143002 \\
\hline 0003000142 & 0004102003 & 0140003002 \\
\hline 0000143020 & 0000204103 & 0000143020 \\
\hline 0003020140 & 0004100203 & 0140003020 \\
\hline 0000143200 & 0000024103 & 0000143200 \\
\hline 0003200140 & 0004100023 & 0140003200 \\
\hline 0000200134 & 0004310200 & 0000200134 \\
\hline 0000130204 & 0000204310 & 0200000134 \\
\hline
\end{tabular}




\begin{tabular}{|c|c|c|}
\hline 0000200143 & 0003410200 & 0000200143 \\
\hline 0000140203 & 0000203410 & 0200000143 \\
\hline 0000200314 & 0004130200 & 0000200314 \\
\hline 0000310204 & 0000204130 & 0200000314 \\
\hline 0000200341 & 0001430200 & 0000200341 \\
\hline 0000340201 & 0000201430 & 0200000341 \\
\hline 0000200413 & 0003140200 & 0000200413 \\
\hline 0000410203 & 0000203140 & 0200000413 \\
\hline 0000200431 & 0001340200 & 0000200431 \\
\hline 0000430201 & 0000201340 & 0200000431 \\
\hline 0000201034 & 0004300201 & 0000201034 \\
\hline 0001030204 & 0000204301 & 0200001034 \\
\hline 0000201043 & 0003400201 & 0000201043 \\
\hline 0001040203 & 0000203401 & 0200001043 \\
\hline 0000201304 & 0004030201 & 0000201304 \\
\hline 0001300204 & 0000204031 & 0200001304 \\
\hline 0000201403 & 0003040201 & 0000201403 \\
\hline 0001400203 & 0000203041 & 0200001403 \\
\hline 0000203014 & 0004100203 & 0000203014 \\
\hline 0003010204 & 0000204103 & 0200003014 \\
\hline 0000203104 & 0004010203 & 0000203104 \\
\hline 0003100204 & 0000204013 & 0200003104 \\
\hline 0000210034 & 0004301200 & 0000210034 \\
\hline 0000030214 & 0001204300 & 0210000034 \\
\hline 0000210043 & 0003401200 & 0000210043 \\
\hline 0000040213 & 0001203400 & 0210000043 \\
\hline 0000210304 & 0004031200 & 0000210304 \\
\hline 0000300214 & 0001204030 & 0210000304 \\
\hline 0000210340 & 0000431200 & 0000210340 \\
\hline 0000340210 & 0001200430 & 0210000340 \\
\hline 0000210403 & 0003041200 & 0000210403 \\
\hline 0000400213 & 0001203040 & 0210000403 \\
\hline 0000210430 & 0000341200 & 0000210430 \\
\hline 0000430210 & 0001200340 & 0210000430 \\
\hline 0000213004 & 0004001203 & 0000213004 \\
\hline 0003000214 & 0001204003 & 0210003004 \\
\hline 0000213040 & 0000401203 & 0000213040 \\
\hline 0003040210 & 0001200403 & 0210003040 \\
\hline 0000213400 & 0000041203 & 0000213400 \\
\hline 0003400210 & 0001200043 & 0210003400 \\
\hline 0000214003 & 0003001204 & 0000214003 \\
\hline 0004000213 & 0001203004 & 0210004003 \\
\hline 0000214030 & 0000301204 & 0000214030 \\
\hline 0004030210 & 0001200304 & 0210004030 \\
\hline 0000214300 & 0000031204 & 0000214300 \\
\hline 0004300210 & 0001200034 & 0210004300 \\
\hline 0000230014 & 0004103200 & 0000230014 \\
\hline 0000010234 & 0003204100 & 0230000014 \\
\hline 0000230041 & 0001403200 & 0000230041 \\
\hline 0000040231 & 0003201400 & 0230000041 \\
\hline
\end{tabular}




\begin{tabular}{|c|c|c|}
\hline 0000230104 & 0004013200 & 0000230104 \\
\hline 0000100234 & 0003204010 & 0230000104 \\
\hline 0000230140 & 0000413200 & 0000230140 \\
\hline 0000140230 & 0003200410 & 0230000140 \\
\hline 0000230401 & 0001043200 & 0000230401 \\
\hline 0000400231 & 0003201040 & 0230000401 \\
\hline 0000230410 & 0000143200 & 0000230410 \\
\hline 0000410230 & 0003200140 & 0230000410 \\
\hline 0000231004 & 0004003201 & 0000231004 \\
\hline 0001000234 & 0003204001 & 0230001004 \\
\hline 0000231040 & 0000403201 & 0000231040 \\
\hline 0001040230 & 0003200401 & 0230001040 \\
\hline 0000231400 & 0000043201 & 0000231400 \\
\hline 0001400230 & 0003200041 & 0230001400 \\
\hline 0000234001 & 0001003204 & 0000234001 \\
\hline 0004000231 & 0003201004 & 0230004001 \\
\hline 0000234010 & 0000103204 & 0000234010 \\
\hline 0004010230 & 0003200104 & 0230004010 \\
\hline 0000234100 & 0000013204 & 0000234100 \\
\hline 0004100230 & 0003200014 & 0230004100 \\
\hline 0000240013 & 0003104200 & 0000240013 \\
\hline 0000010243 & 0004203100 & 0240000013 \\
\hline 0000240031 & 0001304200 & 0000240031 \\
\hline 0000030241 & 0004201300 & 0240000031 \\
\hline 0000240103 & 0003014200 & 0000240103 \\
\hline 0000100243 & 0004203010 & 0240000103 \\
\hline 0000240130 & 0000314200 & 0000240130 \\
\hline 0000130240 & 0004200310 & 0240000130 \\
\hline 0000240301 & 0001034200 & 0000240301 \\
\hline 0000300241 & 0004201030 & 0240000301 \\
\hline 0000240310 & 0000134200 & 0000240310 \\
\hline 0000310240 & 0004200130 & 0240000310 \\
\hline 0000241003 & 0003004201 & 0000241003 \\
\hline 0001000243 & 0004203001 & 0240001003 \\
\hline 0000241030 & 0000304201 & 0000241030 \\
\hline 0001030240 & 0004200301 & 0240001030 \\
\hline 0000241300 & 0000034201 & 0000241300 \\
\hline 0001300240 & 0004200031 & 0240001300 \\
\hline 0000243001 & 0001004203 & 0000243001 \\
\hline 0003000241 & 0004201003 & 0240003001 \\
\hline 0000243010 & 0000104203 & 0000243010 \\
\hline 0003010240 & 0004200103 & 0240003010 \\
\hline 0000243100 & 0000014203 & 0000243100 \\
\hline 0003100240 & 0004200013 & 0240003100 \\
\hline 0000300124 & 0004210300 & 0000300124 \\
\hline 0000120304 & 0000304210 & 0300000124 \\
\hline 0000300142 & 0002410300 & 0000300142 \\
\hline 0000140302 & 0000302410 & 0300000142 \\
\hline 0000300214 & 0004120300 & 0000300214 \\
\hline 0000210304 & 0000304120 & 0300000214 \\
\hline
\end{tabular}




\begin{tabular}{|c|c|c|}
\hline 0000300241 & 0001420300 & 0000300241 \\
\hline 0000240301 & 0000301420 & 0300000241 \\
\hline 0000300412 & 0002140300 & 0000300412 \\
\hline 0000410302 & 0000302140 & 0300000412 \\
\hline 0000300421 & 0001240300 & 0000300421 \\
\hline 0000420301 & 0000301240 & 0300000421 \\
\hline 0000301024 & 0004200301 & 0000301024 \\
\hline 0001020304 & 0000304201 & 0300001024 \\
\hline 0000301042 & 0002400301 & 0000301042 \\
\hline 0001040302 & 0000302401 & 0300001042 \\
\hline 0000301204 & 0004020301 & 0000301204 \\
\hline 0001200304 & 0000304021 & 0300001204 \\
\hline 0000301402 & 0002040301 & 0000301402 \\
\hline 0001400302 & 0000302041 & 0300001402 \\
\hline 0000302014 & 0004100302 & 0000302014 \\
\hline 0002010304 & 0000304102 & 0300002014 \\
\hline 0000302104 & 0004010302 & 0000302104 \\
\hline 0002100304 & 0000304012 & 0300002104 \\
\hline 0000310024 & 0004201300 & 0000310024 \\
\hline 0000020314 & 0001304200 & 0310000024 \\
\hline 0000310042 & 0002401300 & 0000310042 \\
\hline 0000040312 & 0001302400 & 0310000042 \\
\hline 0000310204 & 0004021300 & 0000310204 \\
\hline 0000200314 & 0001304020 & 0310000204 \\
\hline 0000310240 & 0000421300 & 0000310240 \\
\hline 0000240310 & 0001300420 & 0310000240 \\
\hline 0000310402 & 0002041300 & 0000310402 \\
\hline 0000400312 & 0001302040 & 0310000402 \\
\hline 0000310420 & 0000241300 & 0000310420 \\
\hline 0000420310 & 0001300240 & 0310000420 \\
\hline 0000312004 & 0004001302 & 0000312004 \\
\hline 0002000314 & 0001304002 & 0310002004 \\
\hline 0000312040 & 0000401302 & 0000312040 \\
\hline 0002040310 & 0001300402 & 0310002040 \\
\hline 0000312400 & 0000041302 & 0000312400 \\
\hline 0002400310 & 0001300042 & 0310002400 \\
\hline 0000314002 & 0002001304 & 0000314002 \\
\hline 0004000312 & 0001302004 & 0310004002 \\
\hline 0000314020 & 0000201304 & 0000314020 \\
\hline 0004020310 & 0001300204 & 0310004020 \\
\hline 0000314200 & 0000021304 & 0000314200 \\
\hline 0004200310 & 0001300024 & 0310004200 \\
\hline 0000320014 & 0004102300 & 0000320014 \\
\hline 0000010324 & 0002304100 & 0320000014 \\
\hline 0000320041 & 0001402300 & 0000320041 \\
\hline 0000040321 & 0002301400 & 0320000041 \\
\hline 0000320104 & 0004012300 & 0000320104 \\
\hline 0000100324 & 0002304010 & 0320000104 \\
\hline 0000320140 & 0000412300 & 0000320140 \\
\hline 0000140320 & 0002300410 & 0320000140 \\
\hline
\end{tabular}




\begin{tabular}{|c|c|c|}
\hline 0000320401 & 0001042300 & 0000320401 \\
\hline 0000400321 & 0002301040 & 0320000401 \\
\hline 0000320410 & 0000142300 & 0000320410 \\
\hline 0000410320 & 0002300140 & 0320000410 \\
\hline 0000321004 & 0004002301 & 0000321004 \\
\hline 0001000324 & 0002304001 & 0320001004 \\
\hline 0000321040 & 0000402301 & 0000321040 \\
\hline 0001040320 & 0002300401 & 0320001040 \\
\hline 0000321400 & 0000042301 & 0000321400 \\
\hline 0001400320 & 0002300041 & 0320001400 \\
\hline 0000324001 & 0001002304 & 0000324001 \\
\hline 0004000321 & 0002301004 & 0320004001 \\
\hline 0000324010 & 0000102304 & 0000324010 \\
\hline 0004010320 & 0002300104 & 0320004010 \\
\hline 0000324100 & 0000012304 & 0000324100 \\
\hline 0004100320 & 0002300014 & 0320004100 \\
\hline 0000340012 & 0002104300 & 0000340012 \\
\hline 0000010342 & 0004302100 & 0340000012 \\
\hline 0000340021 & 0001204300 & 0000340021 \\
\hline 0000020341 & 0004301200 & 0340000021 \\
\hline 0000340102 & 0002014300 & 0000340102 \\
\hline 0000100342 & 0004302010 & 0340000102 \\
\hline 0000340120 & 0000214300 & 0000340120 \\
\hline 0000120340 & 0004300210 & 0340000120 \\
\hline 0000340201 & 0001024300 & 0000340201 \\
\hline 0000200341 & 0004301020 & 0340000201 \\
\hline 0000340210 & 0000124300 & 0000340210 \\
\hline 0000210340 & 0004300120 & 0340000210 \\
\hline 0000341002 & 0002004301 & 0000341002 \\
\hline 0001000342 & 0004302001 & 0340001002 \\
\hline 0000341020 & 0000204301 & 0000341020 \\
\hline 0001020340 & 0004300201 & 0340001020 \\
\hline 0000341200 & 0000024301 & 0000341200 \\
\hline 0001200340 & 0004300021 & 0340001200 \\
\hline 0000342001 & 0001004302 & 0000342001 \\
\hline 0002000341 & 0004301002 & 0340002001 \\
\hline 0000342010 & 0000104302 & 0000342010 \\
\hline 0002010340 & 0004300102 & 0340002010 \\
\hline 0000342100 & 0000014302 & 0000342100 \\
\hline 0002100340 & 0004300012 & 0340002100 \\
\hline 0000400123 & 0003210400 & 0000400123 \\
\hline 0000120403 & 0000403210 & 0400000123 \\
\hline 0000400132 & 0002310400 & 0000400132 \\
\hline 0000130402 & 0000402310 & 0400000132 \\
\hline 0000400213 & 0003120400 & 0000400213 \\
\hline 0000210403 & 0000403120 & 0400000213 \\
\hline 0000400231 & 0001320400 & 0000400231 \\
\hline 0000230401 & 0000401320 & 0400000231 \\
\hline 0000400312 & 0002130400 & 0000400312 \\
\hline 0000310402 & 0000402130 & 0400000312 \\
\hline
\end{tabular}




\begin{tabular}{|c|c|c|}
\hline 0000400321 & 0001230400 & 0000400321 \\
\hline 0000320401 & 0000401230 & 0400000321 \\
\hline 0000401023 & 0003200401 & 0000401023 \\
\hline 0001020403 & 0000403201 & 0400001023 \\
\hline 0000401032 & 0002300401 & 0000401032 \\
\hline 0001030402 & 0000402301 & 0400001032 \\
\hline 0000401203 & 0003020401 & 0000401203 \\
\hline 0001200403 & 0000403021 & 0400001203 \\
\hline 0000401302 & 0002030401 & 0000401302 \\
\hline 0001300402 & 0000402031 & 0400001302 \\
\hline 0000402013 & 0003100402 & 0000402013 \\
\hline 0002010403 & 0000403102 & 0400002013 \\
\hline 0000402103 & 0003010402 & 0000402103 \\
\hline 0002100403 & 0000403012 & 0400002103 \\
\hline 0000410023 & 0003201400 & 0000410023 \\
\hline 0000020413 & 0001403200 & 0410000023 \\
\hline 0000410032 & 0002301400 & 0000410032 \\
\hline 0000030412 & 0001402300 & 0410000032 \\
\hline 0000410203 & 0003021400 & 0000410203 \\
\hline 0000200413 & 0001403020 & 0410000203 \\
\hline 0000410230 & 0000321400 & 0000410230 \\
\hline 0000230410 & 0001400320 & 0410000230 \\
\hline 0000410302 & 0002031400 & 0000410302 \\
\hline 0000300412 & 0001402030 & 0410000302 \\
\hline 0000410320 & 0000231400 & 0000410320 \\
\hline 0000320410 & 0001400230 & 0410000320 \\
\hline 0000412003 & 0003001402 & 0000412003 \\
\hline 0002000413 & 0001403002 & 0410002003 \\
\hline 0000412030 & 0000301402 & 0000412030 \\
\hline 0002030410 & 0001400302 & 0410002030 \\
\hline 0000412300 & 0000031402 & 0000412300 \\
\hline 0002300410 & 0001400032 & 0410002300 \\
\hline 0000413002 & 0002001403 & 0000413002 \\
\hline 0003000412 & 0001402003 & 0410003002 \\
\hline 0000413020 & 0000201403 & 0000413020 \\
\hline 0003020410 & 0001400203 & 0410003020 \\
\hline 0000413200 & 0000021403 & 0000413200 \\
\hline 0003200410 & 0001400023 & 0410003200 \\
\hline 0000420013 & 0003102400 & 0000420013 \\
\hline 0000010423 & 0002403100 & 0420000013 \\
\hline 0000420031 & 0001302400 & 0000420031 \\
\hline 0000030421 & 0002401300 & 0420000031 \\
\hline 0000420103 & 0003012400 & 0000420103 \\
\hline 0000100423 & 0002403010 & 0420000103 \\
\hline 0000420130 & 0000312400 & 0000420130 \\
\hline 0000130420 & 0002400310 & 0420000130 \\
\hline 0000420301 & 0001032400 & 0000420301 \\
\hline 0000300421 & 0002401030 & 0420000301 \\
\hline 0000420310 & 0000132400 & 0000420310 \\
\hline 0000310420 & 0002400130 & 0420000310 \\
\hline
\end{tabular}




\begin{tabular}{|c|c|c|}
\hline 0000421003 & 0003002401 & 0000421003 \\
\hline 0001000423 & 0002403001 & 0420001003 \\
\hline 0000421030 & 0000302401 & 0000421030 \\
\hline 0001030420 & 0002400301 & 0420001030 \\
\hline 0000421300 & 0000032401 & 0000421300 \\
\hline 0001300420 & 0002400031 & 0420001300 \\
\hline 0000423001 & 0001002403 & 0000423001 \\
\hline 0003000421 & 0002401003 & 0420003001 \\
\hline 0000423010 & 0000102403 & 0000423010 \\
\hline 0003010420 & 0002400103 & 0420003010 \\
\hline 0000423100 & 0000012403 & 0000423100 \\
\hline 0003100420 & 0002400013 & 0420003100 \\
\hline 0000430012 & 0002103400 & 0000430012 \\
\hline 0000010432 & 0003402100 & 0430000012 \\
\hline 0000430021 & 0001203400 & 0000430021 \\
\hline 0000020431 & 0003401200 & 0430000021 \\
\hline 0000430102 & 0002013400 & 0000430102 \\
\hline 0000100432 & 0003402010 & 0430000102 \\
\hline 0000430120 & 0000213400 & 0000430120 \\
\hline 0000120430 & 0003400210 & 0430000120 \\
\hline 0000430201 & 0001023400 & 0000430201 \\
\hline 0000200431 & 0003401020 & 0430000201 \\
\hline 0000430210 & 0000123400 & 0000430210 \\
\hline 0000210430 & 0003400120 & 0430000210 \\
\hline 0000431002 & 0002003401 & 0000431002 \\
\hline 0001000432 & 0003402001 & 0430001002 \\
\hline 0000431020 & 0000203401 & 0000431020 \\
\hline 0001020430 & 0003400201 & 0430001020 \\
\hline 0000431200 & 0000023401 & 0000431200 \\
\hline 0001200430 & 0003400021 & 0430001200 \\
\hline 0000432001 & 0001003402 & 0000432001 \\
\hline 0002000431 & 0003401002 & 0430002001 \\
\hline 0000432010 & 0000103402 & 0000432010 \\
\hline 0002010430 & 0003400102 & 0430002010 \\
\hline 0000432100 & 0000013402 & 0000432100 \\
\hline 0002100430 & 0003400012 & 0430002100 \\
\hline 0001020034 & 0004302010 & 0001020034 \\
\hline 0000031024 & 0002014300 & 1020000034 \\
\hline 0001020043 & 0003402010 & 0001020043 \\
\hline 0000041023 & 0002013400 & 1020000043 \\
\hline 0001020304 & 0004032010 & 0001020304 \\
\hline 0000301024 & 0002014030 & 1020000304 \\
\hline 0001020340 & 0000432010 & 0001020340 \\
\hline 0000341020 & 0002010430 & 1020000340 \\
\hline 0001020403 & 0003042010 & 0001020403 \\
\hline 0000401023 & 0002013040 & 1020000403 \\
\hline 0001020430 & 0000342010 & 0001020430 \\
\hline 0000431020 & 0002010340 & 1020000430 \\
\hline 0001023004 & 0004002013 & 0001023004 \\
\hline 0003001024 & 0002014003 & 1020003004 \\
\hline
\end{tabular}




\begin{tabular}{|c|c|c|}
\hline 0001023040 & 0000402013 & 0001023040 \\
\hline 0003041020 & 0002010403 & 1020003040 \\
\hline 0001023400 & 0000042013 & 0001023400 \\
\hline 0003401020 & 0002010043 & 1020003400 \\
\hline 0001024003 & 0003002014 & 0001024003 \\
\hline 0004001023 & 0002013004 & 1020004003 \\
\hline 0001024030 & 0000302014 & 0001024030 \\
\hline 0004031020 & 0002010304 & 1020004030 \\
\hline 0001024300 & 0000032014 & 0001024300 \\
\hline 0004301020 & 0002010034 & 1020004300 \\
\hline 0001030024 & 0004203010 & 0001030024 \\
\hline 0000021034 & 0003014200 & 1030000024 \\
\hline 0001030042 & 0002403010 & 0001030042 \\
\hline 0000041032 & 0003012400 & 1030000042 \\
\hline 0001030204 & 0004023010 & 0001030204 \\
\hline 0000201034 & 0003014020 & 1030000204 \\
\hline 0001030240 & 0000423010 & 0001030240 \\
\hline 0000241030 & 0003010420 & 1030000240 \\
\hline 0001030402 & 0002043010 & 0001030402 \\
\hline 0000401032 & 0003012040 & 1030000402 \\
\hline 0001030420 & 0000243010 & 0001030420 \\
\hline 0000421030 & 0003010240 & 1030000420 \\
\hline 0001032004 & 0004003012 & 0001032004 \\
\hline 0002001034 & 0003014002 & 1030002004 \\
\hline 0001032040 & 0000403012 & 0001032040 \\
\hline 0002041030 & 0003010402 & 1030002040 \\
\hline 0001032400 & 0000043012 & 0001032400 \\
\hline 0002401030 & 0003010042 & 1030002400 \\
\hline 0001034002 & 0002003014 & 0001034002 \\
\hline 0004001032 & 0003012004 & 1030004002 \\
\hline 0001034020 & 0000203014 & 0001034020 \\
\hline 0004021030 & 0003010204 & 1030004020 \\
\hline 0001034200 & 0000023014 & 0001034200 \\
\hline 0004201030 & 0003010024 & 1030004200 \\
\hline 0001040023 & 0003204010 & 0001040023 \\
\hline 0000021043 & 0004013200 & 1040000023 \\
\hline 0001040032 & 0002304010 & 0001040032 \\
\hline 0000031042 & 0004012300 & 1040000032 \\
\hline 0001040203 & 0003024010 & 0001040203 \\
\hline 0000201043 & 0004013020 & 1040000203 \\
\hline 0001040230 & 0000324010 & 0001040230 \\
\hline 0000231040 & 0004010320 & 1040000230 \\
\hline 0001040302 & 0002034010 & 0001040302 \\
\hline 0000301042 & 0004012030 & 1040000302 \\
\hline 0001040320 & 0000234010 & 0001040320 \\
\hline 0000321040 & 0004010230 & 1040000320 \\
\hline 0001042003 & 0003004012 & 0001042003 \\
\hline 0002001043 & 0004013002 & 1040002003 \\
\hline 0001042030 & 0000304012 & 0001042030 \\
\hline 0002031040 & 0004010302 & 1040002030 \\
\hline
\end{tabular}




\begin{tabular}{|c|c|c|}
\hline 0001042300 & 0000034012 & 0001042300 \\
\hline 0002301040 & 0004010032 & 1040002300 \\
\hline 0001043002 & 0002004013 & 0001043002 \\
\hline 0003001042 & 0004012003 & 1040003002 \\
\hline 0001043020 & 0000204013 & 0001043020 \\
\hline 0003021040 & 0004010203 & 1040003020 \\
\hline 0001043200 & 0000024013 & 0001043200 \\
\hline 0003201040 & 0004010023 & 1040003200 \\
\hline 0000001234 & 0004003210 & 0001230004 \\
\hline 0000001234 & 0003214000 & 1230000004 \\
\hline 0001230040 & 0000403210 & 0001230040 \\
\hline 0000041230 & 0003210400 & 1230000040 \\
\hline 0001230400 & 0000043210 & 0001230400 \\
\hline 0000401230 & 0003210040 & 1230000400 \\
\hline 0001234000 & 0000003214 & 0001234000 \\
\hline 0004001230 & 0000003214 & 1230004000 \\
\hline 0000001243 & 0003004210 & 0001240003 \\
\hline 0000001243 & 0004213000 & 1240000003 \\
\hline 0001240030 & 0000304210 & 0001240030 \\
\hline 0000031240 & 0004210300 & 1240000030 \\
\hline 0001240300 & 0000034210 & 0001240300 \\
\hline 0000301240 & 0004210030 & 1240000300 \\
\hline 0001243000 & 0000004213 & 0001243000 \\
\hline 0003001240 & 0000004213 & 1240003000 \\
\hline 0000001324 & 0004002310 & 0001320004 \\
\hline 0000001324 & 0002314000 & 1320000004 \\
\hline 0001320040 & 0000402310 & 0001320040 \\
\hline 0000041320 & 0002310400 & 1320000040 \\
\hline 0001320400 & 0000042310 & 0001320400 \\
\hline 0000401320 & 0002310040 & 1320000400 \\
\hline 0001324000 & 0000002314 & 0001324000 \\
\hline 0004001320 & 0000002314 & 1320004000 \\
\hline 0000001342 & 0002004310 & 0001340002 \\
\hline 0000001342 & 0004312000 & 1340000002 \\
\hline 0001340020 & 0000204310 & 0001340020 \\
\hline 0000021340 & 0004310200 & 1340000020 \\
\hline 0001340200 & 0000024310 & 0001340200 \\
\hline 0000201340 & 0004310020 & 1340000200 \\
\hline 0001342000 & 0000004312 & 0001342000 \\
\hline 0002001340 & 0000004312 & 1340002000 \\
\hline 0000001423 & 0003002410 & 0001420003 \\
\hline 0000001423 & 0002413000 & 1420000003 \\
\hline 0001420030 & 0000302410 & 0001420030 \\
\hline 0000031420 & 0002410300 & 1420000030 \\
\hline 0001420300 & 0000032410 & 0001420300 \\
\hline 0000301420 & 0002410030 & 1420000300 \\
\hline 0001423000 & 0000002413 & 0001423000 \\
\hline 0003001420 & 0000002413 & 1420003000 \\
\hline 0000001432 & 0002003410 & 0001430002 \\
\hline 0000001432 & 0003412000 & 1430000002 \\
\hline
\end{tabular}




\begin{tabular}{|c|c|c|}
\hline 0001430020 & 0000203410 & 0001430020 \\
\hline 0000021430 & 0003410200 & 1430000020 \\
\hline 0001430200 & 0000023410 & 0001430200 \\
\hline 0000201430 & 0003410020 & 1430000200 \\
\hline 0001432000 & 0000003412 & 0001432000 \\
\hline 0002001430 & 0000003412 & 1430002000 \\
\hline 0002030014 & 0004103020 & 0002030014 \\
\hline 0000012034 & 0003024100 & 2030000014 \\
\hline 0002030041 & 0001403020 & 0002030041 \\
\hline 0000042031 & 0003021400 & 2030000041 \\
\hline 0002030104 & 0004013020 & 0002030104 \\
\hline 0000102034 & 0003024010 & 2030000104 \\
\hline 0002030140 & 0000413020 & 0002030140 \\
\hline 0000142030 & 0003020410 & 2030000140 \\
\hline 0002030401 & 0001043020 & 0002030401 \\
\hline 0000402031 & 0003021040 & 2030000401 \\
\hline 0002030410 & 0000143020 & 0002030410 \\
\hline 0000412030 & 0003020140 & 2030000410 \\
\hline 0002031004 & 0004003021 & 0002031004 \\
\hline 0001002034 & 0003024001 & 2030001004 \\
\hline 0002031040 & 0000403021 & 0002031040 \\
\hline 0001042030 & 0003020401 & 2030001040 \\
\hline 0002031400 & 0000043021 & 0002031400 \\
\hline 0001402030 & 0003020041 & 2030001400 \\
\hline 0002034001 & 0001003024 & 0002034001 \\
\hline 0004002031 & 0003021004 & 2030004001 \\
\hline 0002034010 & 0000103024 & 0002034010 \\
\hline 0004012030 & 0003020104 & 2030004010 \\
\hline 0002034100 & 0000013024 & 0002034100 \\
\hline 0004102030 & 0003020014 & 2030004100 \\
\hline 0002040013 & 0003104020 & 0002040013 \\
\hline 0000012043 & 0004023100 & 2040000013 \\
\hline 0002040031 & 0001304020 & 0002040031 \\
\hline 0000032041 & 0004021300 & 2040000031 \\
\hline 0002040103 & 0003014020 & 0002040103 \\
\hline 0000102043 & 0004023010 & 2040000103 \\
\hline 0002040130 & 0000314020 & 0002040130 \\
\hline 0000132040 & 0004020310 & 2040000130 \\
\hline 0002040301 & 0001034020 & 0002040301 \\
\hline 0000302041 & 0004021030 & 2040000301 \\
\hline 0002040310 & 0000134020 & 0002040310 \\
\hline 0000312040 & 0004020130 & 2040000310 \\
\hline 0002041003 & 0003004021 & 0002041003 \\
\hline 0001002043 & 0004023001 & 2040001003 \\
\hline 0002041030 & 0000304021 & 0002041030 \\
\hline 0001032040 & 0004020301 & 2040001030 \\
\hline 0002041300 & 0000034021 & 0002041300 \\
\hline 0001302040 & 0004020031 & 2040001300 \\
\hline 0002043001 & 0001004023 & 0002043001 \\
\hline 0003002041 & 0004021003 & 2040003001 \\
\hline
\end{tabular}




\begin{tabular}{|c|c|c|}
\hline 0002043010 & 0000104023 & 0002043010 \\
\hline 0003012040 & 0004020103 & 2040003010 \\
\hline 0002043100 & 0000014023 & 0002043100 \\
\hline 0003102040 & 0004020013 & 2040003100 \\
\hline 0000002134 & 0004003120 & 0002130004 \\
\hline 0000002134 & 0003124000 & 2130000004 \\
\hline 0002130040 & 0000403120 & 0002130040 \\
\hline 0000042130 & 0003120400 & 2130000040 \\
\hline 0002130400 & 0000043120 & 0002130400 \\
\hline 0000402130 & 0003120040 & 2130000400 \\
\hline 0002134000 & 0000003124 & 0002134000 \\
\hline 0004002130 & 0000003124 & 2130004000 \\
\hline 0000002143 & 0003004120 & 0002140003 \\
\hline 0000002143 & 0004123000 & 2140000003 \\
\hline 0002140030 & 0000304120 & 0002140030 \\
\hline 0000032140 & 0004120300 & 2140000030 \\
\hline 0002140300 & 0000034120 & 0002140300 \\
\hline 0000302140 & 0004120030 & 2140000300 \\
\hline 0002143000 & 0000004123 & 0002143000 \\
\hline 0003002140 & 0000004123 & 2140003000 \\
\hline 0000002341 & 0001004320 & 0002340001 \\
\hline 0000002341 & 0004321000 & 2340000001 \\
\hline 0002340010 & 0000104320 & 0002340010 \\
\hline 0000012340 & 0004320100 & 2340000010 \\
\hline 0002340100 & 0000014320 & 0002340100 \\
\hline 0000102340 & 0004320010 & 2340000100 \\
\hline 0002341000 & 0000004321 & 0002341000 \\
\hline 0001002340 & 0000004321 & 2340001000 \\
\hline 0000002431 & 0001003420 & 0002430001 \\
\hline 0000002431 & 0003421000 & 2430000001 \\
\hline 0002430010 & 0000103420 & 0002430010 \\
\hline 0000012430 & 0003420100 & 2430000010 \\
\hline 0002430100 & 0000013420 & 0002430100 \\
\hline 0000102430 & 0003420010 & 2430000100 \\
\hline 0002431000 & 0000003421 & 0002431000 \\
\hline 0001002430 & 0000003421 & 2430001000 \\
\hline 0003040012 & 0002104030 & 0003040012 \\
\hline 0000013042 & 0004032100 & 3040000012 \\
\hline 0003040021 & 0001204030 & 0003040021 \\
\hline 0000023041 & 0004031200 & 3040000021 \\
\hline 0003040102 & 0002014030 & 0003040102 \\
\hline 0000103042 & 0004032010 & 3040000102 \\
\hline 0003040120 & 0000214030 & 0003040120 \\
\hline 0000123040 & 0004030210 & 3040000120 \\
\hline 0003040201 & 0001024030 & 0003040201 \\
\hline 0000203041 & 0004031020 & 3040000201 \\
\hline 0003040210 & 0000124030 & 0003040210 \\
\hline 0000213040 & 0004030120 & 3040000210 \\
\hline 0003041002 & 0002004031 & 0003041002 \\
\hline 0001003042 & 0004032001 & 3040001002 \\
\hline
\end{tabular}




\begin{tabular}{|c|c|c|}
\hline 0003041020 & 0000204031 & 0003041020 \\
\hline 0001023040 & 0004030201 & 3040001020 \\
\hline 0003041200 & 0000024031 & 0003041200 \\
\hline 0001203040 & 0004030021 & 3040001200 \\
\hline 0003042001 & 0001004032 & 0003042001 \\
\hline 0002003041 & 0004031002 & 3040002001 \\
\hline 0003042010 & 0000104032 & 0003042010 \\
\hline 0002013040 & 0004030102 & 3040002010 \\
\hline 0003042100 & 0000014032 & 0003042100 \\
\hline 0002103040 & 0004030012 & 3040002100 \\
\hline 0000003142 & 0002004130 & 0003140002 \\
\hline 0000003142 & 0004132000 & 3140000002 \\
\hline 0003140020 & 0000204130 & 0003140020 \\
\hline 0000023140 & 0004130200 & 3140000020 \\
\hline 0003140200 & 0000024130 & 0003140200 \\
\hline 0000203140 & 0004130020 & 3140000200 \\
\hline 0003142000 & 0000004132 & 0003142000 \\
\hline 0002003140 & 0000004132 & 3140002000 \\
\hline 0000003241 & 0001004230 & 0003240001 \\
\hline 0000003241 & 0004231000 & 3240000001 \\
\hline 0003240010 & 0000104230 & 0003240010 \\
\hline 0000013240 & 0004230100 & 3240000010 \\
\hline 0003240100 & 0000014230 & 0003240100 \\
\hline 0000103240 & 0004230010 & 3240000100 \\
\hline 0003241000 & 0000004231 & 0003241000 \\
\hline 0001003240 & 0000004231 & 3240001000 \\
\hline 0010020034 & 1004302000 & 0010020034 \\
\hline 0010030024 & 1002004300 & 0020010034 \\
\hline 0010020043 & 1003402000 & 0010020043 \\
\hline 0010040023 & 1002003400 & 0020010043 \\
\hline 0010020304 & 1004032000 & 0010020304 \\
\hline 0010300024 & 1002004030 & 0020010304 \\
\hline 0010020340 & 0432001000 & 0010020340 \\
\hline 0010340020 & 0431002000 & 0020010340 \\
\hline 0010020403 & 1003042000 & 0010020403 \\
\hline 0010400023 & 1002003040 & 0020010403 \\
\hline 0010020430 & 0342001000 & 0010020430 \\
\hline 0010430020 & 0341002000 & 0020010430 \\
\hline 0010023004 & 1004002003 & 0010023004 \\
\hline 0013000024 & 1002004003 & 0020013004 \\
\hline 0010023040 & 0402001003 & 0010023040 \\
\hline 0013040020 & 0401002003 & 0020013040 \\
\hline 0010023400 & 0042001003 & 0010023400 \\
\hline 0013400020 & 0041002003 & 0020013400 \\
\hline 0010024003 & 1003002004 & 0010024003 \\
\hline 0014000023 & 1002003004 & 0020014003 \\
\hline 0010024030 & 0302001004 & 0010024030 \\
\hline 0014030020 & 0301002004 & 0020014030 \\
\hline 0010024300 & 0032001004 & 0010024300 \\
\hline 0014300020 & 0031002004 & 0020014300 \\
\hline
\end{tabular}




\begin{tabular}{|c|c|c|}
\hline 0010030024 & 1004203000 & 0010030024 \\
\hline 0010020034 & 1003004200 & 0030010024 \\
\hline 0010030042 & 1002403000 & 0010030042 \\
\hline 0010040032 & 1003002400 & 0030010042 \\
\hline 0010030204 & 1004023000 & 0010030204 \\
\hline 0010200034 & 1003004020 & 0030010204 \\
\hline 0010030240 & 0423001000 & 0010030240 \\
\hline 0010240030 & 0421003000 & 0030010240 \\
\hline 0010030402 & 1002043000 & 0010030402 \\
\hline 0010400032 & 1003002040 & 0030010402 \\
\hline 0010030420 & 0243001000 & 0010030420 \\
\hline 0010420030 & 0241003000 & 0030010420 \\
\hline 0010032004 & 1004003002 & 0010032004 \\
\hline 0012000034 & 1003004002 & 0030012004 \\
\hline 0010032040 & 0403001002 & 0010032040 \\
\hline 0012040030 & 0401003002 & 0030012040 \\
\hline 0010032400 & 0043001002 & 0010032400 \\
\hline 0012400030 & 0041003002 & 0030012400 \\
\hline 0010034002 & 1002003004 & 0010034002 \\
\hline 0014000032 & 1003002004 & 0030014002 \\
\hline 0010034020 & 0203001004 & 0010034020 \\
\hline 0014020030 & 0201003004 & 0030014020 \\
\hline 0010034200 & 0023001004 & 0010034200 \\
\hline 0014200030 & 0021003004 & 0030014200 \\
\hline 0010040023 & 1003204000 & 0010040023 \\
\hline 0010020043 & 1004003200 & 0040010023 \\
\hline 0010040032 & 1002304000 & 0010040032 \\
\hline 0010030042 & 1004002300 & 0040010032 \\
\hline 0010040203 & 1003024000 & 0010040203 \\
\hline 0010200043 & 1004003020 & 0040010203 \\
\hline 0010040230 & 0324001000 & 0010040230 \\
\hline 0010230040 & 0321004000 & 0040010230 \\
\hline 0010040302 & 1002034000 & 0010040302 \\
\hline 0010300042 & 1004002030 & 0040010302 \\
\hline 0010040320 & 0234001000 & 0010040320 \\
\hline 0010320040 & 0231004000 & 0040010320 \\
\hline 0010042003 & 1003004002 & 0010042003 \\
\hline 0012000043 & 1004003002 & 0040012003 \\
\hline 0010042030 & 0304001002 & 0010042030 \\
\hline 0012030040 & 0301004002 & 0040012030 \\
\hline 0010042300 & 0034001002 & 0010042300 \\
\hline 0012300040 & 0031004002 & 0040012300 \\
\hline 0010043002 & 1002004003 & 0010043002 \\
\hline 0013000042 & 1004002003 & 0040013002 \\
\hline 0010043020 & 0204001003 & 0010043020 \\
\hline 0013020040 & 0201004003 & 0040013020 \\
\hline 0010043200 & 0024001003 & 0010043200 \\
\hline 0013200040 & 0021004003 & 0040013200 \\
\hline 0010200034 & 0201004300 & 0010200034 \\
\hline 0010030204 & 0204301000 & 0200010034 \\
\hline
\end{tabular}




\begin{tabular}{|c|c|c|}
\hline 0010200043 & 0201003400 & 0010200043 \\
\hline 0010040203 & 0203401000 & 0200010043 \\
\hline 0010200304 & 0201004030 & 0010200304 \\
\hline 0010300204 & 0204031000 & 0200010304 \\
\hline 0010200340 & 0201000430 & 0010200340 \\
\hline 0010340200 & 0200431000 & 0200010340 \\
\hline 0010200403 & 0201003040 & 0010200403 \\
\hline 0010400203 & 0203041000 & 0200010403 \\
\hline 0010200430 & 0201000340 & 0010200430 \\
\hline 0010430200 & 0200341000 & 0200010430 \\
\hline 0010203004 & 0201004003 & 0010203004 \\
\hline 0013000204 & 0204001003 & 0200013004 \\
\hline 0010203040 & 0201000403 & 0010203040 \\
\hline 0013040200 & 0200401003 & 0200013040 \\
\hline 0010203400 & 0040201003 & 0010203400 \\
\hline 0013400200 & 0041000203 & 0200013400 \\
\hline 0010204003 & 0201003004 & 0010204003 \\
\hline 0014000203 & 0203001004 & 0200014003 \\
\hline 0010204030 & 0201000304 & 0010204030 \\
\hline 0014030200 & 0200301004 & 0200014030 \\
\hline 0010204300 & 0030201004 & 0010204300 \\
\hline 0014300200 & 0031000204 & 0200014300 \\
\hline 0000010234 & 1004003200 & 0010230004 \\
\hline 0000230014 & 1003204000 & 0230010004 \\
\hline 0010230040 & 0403201000 & 0010230040 \\
\hline 0010040230 & 0401003200 & 0230010040 \\
\hline 0010230400 & 0043201000 & 0010230400 \\
\hline 0010400230 & 0041003200 & 0230010400 \\
\hline 0010234000 & 0003201004 & 0010234000 \\
\hline 0014000230 & 0001003204 & 0230014000 \\
\hline 0000010243 & 1003004200 & 0010240003 \\
\hline 0000240013 & 1004203000 & 0240010003 \\
\hline 0010240030 & 0304201000 & 0010240030 \\
\hline 0010030240 & 0301004200 & 0240010030 \\
\hline 0010240300 & 0034201000 & 0010240300 \\
\hline 0010300240 & 0031004200 & 0240010300 \\
\hline 0010243000 & 0004201003 & 0010243000 \\
\hline 0013000240 & 0001004203 & 0240013000 \\
\hline 0010300024 & 0301004200 & 0010300024 \\
\hline 0010020304 & 0304201000 & 0300010024 \\
\hline 0010300042 & 0301002400 & 0010300042 \\
\hline 0010040302 & 0302401000 & 0300010042 \\
\hline 0010300204 & 0301004020 & 0010300204 \\
\hline 0010200304 & 0304021000 & 0300010204 \\
\hline 0010300240 & 0301000420 & 0010300240 \\
\hline 0010240300 & 0300421000 & 0300010240 \\
\hline 0010300402 & 0301002040 & 0010300402 \\
\hline 0010400302 & 0302041000 & 0300010402 \\
\hline 0010300420 & 0240301000 & 0010300420 \\
\hline 0010420300 & 0241000300 & 0300010420 \\
\hline
\end{tabular}




\begin{tabular}{|c|c|c|}
\hline 0010302004 & 0301004002 & 0010302004 \\
\hline 0012000304 & 0304001002 & 0300012004 \\
\hline 0010302040 & 0301000402 & 0010302040 \\
\hline 0012040300 & 0300401002 & 0300012040 \\
\hline 0010302400 & 0040301002 & 0010302400 \\
\hline 0012400300 & 0041000302 & 0300012400 \\
\hline 0010304002 & 0301002004 & 0010304002 \\
\hline 0014000302 & 0302001004 & 0300014002 \\
\hline 0010304020 & 0200301004 & 0010304020 \\
\hline 0014020300 & 0201000304 & 0300014020 \\
\hline 0010304200 & 0020301004 & 0010304200 \\
\hline 0014200300 & 0021000304 & 0300014200 \\
\hline 0000010324 & 1004002300 & 0010320004 \\
\hline 0000320014 & 1002304000 & 0320010004 \\
\hline 0010320040 & 0402301000 & 0010320040 \\
\hline 0010040320 & 0401002300 & 0320010040 \\
\hline 0010320400 & 0042301000 & 0010320400 \\
\hline 0010400320 & 0041002300 & 0320010400 \\
\hline 0010324000 & 0002301004 & 0010324000 \\
\hline 0014000320 & 0001002304 & 0320014000 \\
\hline 0000010342 & 1002004300 & 0010340002 \\
\hline 0000340012 & 1004302000 & 0340010002 \\
\hline 0010340020 & 0204301000 & 0010340020 \\
\hline 0010020340 & 0201004300 & 0340010020 \\
\hline 0010340200 & 0024301000 & 0010340200 \\
\hline 0010200340 & 0021004300 & 0340010200 \\
\hline 0010342000 & 0004301002 & 0010342000 \\
\hline 0012000340 & 0001004302 & 0340012000 \\
\hline 0010400023 & 0401003200 & 0010400023 \\
\hline 0010020403 & 0403201000 & 0400010023 \\
\hline 0010400032 & 0401002300 & 0010400032 \\
\hline 0010030402 & 0402301000 & 0400010032 \\
\hline 0010400203 & 0401003020 & 0010400203 \\
\hline 0010200403 & 0403021000 & 0400010203 \\
\hline 0010400230 & 0320401000 & 0010400230 \\
\hline 0010230400 & 0321000400 & 0400010230 \\
\hline 0010400302 & 0401002030 & 0010400302 \\
\hline 0010300402 & 0402031000 & 0400010302 \\
\hline 0010400320 & 0230401000 & 0010400320 \\
\hline 0010320400 & 0231000400 & 0400010320 \\
\hline 0010402003 & 0401003002 & 0010402003 \\
\hline 0012000403 & 0403001002 & 0400012003 \\
\hline 0010402030 & 0300401002 & 0010402030 \\
\hline 0012030400 & 0301000402 & 0400012030 \\
\hline 0010402300 & 0030401002 & 0010402300 \\
\hline 0012300400 & 0031000402 & 0400012300 \\
\hline 0010403002 & 0401002003 & 0010403002 \\
\hline 0013000402 & 0402001003 & 0400013002 \\
\hline 0010403020 & 0200401003 & 0010403020 \\
\hline 0013020400 & 0201000403 & 0400013020 \\
\hline
\end{tabular}




\begin{tabular}{|c|c|c|}
\hline 0010403200 & 0020401003 & 0010403200 \\
\hline 0013200400 & 0021000403 & 0400013200 \\
\hline 0000010423 & 1003002400 & 0010420003 \\
\hline 0000420013 & 1002403000 & 0420010003 \\
\hline 0010420030 & 0302401000 & 0010420030 \\
\hline 0010030420 & 0301002400 & 0420010030 \\
\hline 0010420300 & 0032401000 & 0010420300 \\
\hline 0010300420 & 0031002400 & 0420010300 \\
\hline 0010423000 & 0002401003 & 0010423000 \\
\hline 0013000420 & 0001002403 & 0420013000 \\
\hline 0000010432 & 1002003400 & 0010430002 \\
\hline 0000430012 & 1003402000 & 0430010002 \\
\hline 0010430020 & 0203401000 & 0010430020 \\
\hline 0010020430 & 0201003400 & 0430010020 \\
\hline 0010430200 & 0023401000 & 0010430200 \\
\hline 0010200430 & 0021003400 & 0430010200 \\
\hline 0010432000 & 0003401002 & 0010432000 \\
\hline 0012000430 & 0001003402 & 0430012000 \\
\hline 0012000034 & 0021004300 & 0012000034 \\
\hline 0010032004 & 0024301000 & 2000010034 \\
\hline 0012000043 & 0021003400 & 0012000043 \\
\hline 0010042003 & 0023401000 & 2000010043 \\
\hline 0012000304 & 0021004030 & 0012000304 \\
\hline 0010302004 & 0024031000 & 2000010304 \\
\hline 0012000340 & 0021000430 & 0012000340 \\
\hline 0010342000 & 0020431000 & 2000010340 \\
\hline 0012000403 & 0021003040 & 0012000403 \\
\hline 0010402003 & 0023041000 & 2000010403 \\
\hline 0012000430 & 0021000340 & 0012000430 \\
\hline 0010432000 & 0020341000 & 2000010430 \\
\hline 0012003004 & 0021004003 & 0012003004 \\
\hline 0013002004 & 0024001003 & 2000013004 \\
\hline 0012003040 & 0021000403 & 0012003040 \\
\hline 0013042000 & 0020401003 & 2000013040 \\
\hline 0012003400 & 0021000043 & 0012003400 \\
\hline 0013402000 & 0020041003 & 2000013400 \\
\hline 0012004003 & 0021003004 & 0012004003 \\
\hline 0014002003 & 0023001004 & 2000014003 \\
\hline 0012004030 & 0021000304 & 0012004030 \\
\hline 0014032000 & 0020301004 & 2000014030 \\
\hline 0012004300 & 0021000034 & 0012004300 \\
\hline 0014302000 & 0020031004 & 2000014300 \\
\hline 0000012034 & 1004003020 & 0012030004 \\
\hline 0002030014 & 1003024000 & 2030010004 \\
\hline 0012030040 & 0403021000 & 0012030040 \\
\hline 0010042030 & 0401003020 & 2030010040 \\
\hline 0012030400 & 0043021000 & 0012030400 \\
\hline 0010402030 & 0041003020 & 2030010400 \\
\hline 0012034000 & 0003021004 & 0012034000 \\
\hline 0014002030 & 0001003024 & 2030014000 \\
\hline
\end{tabular}




\begin{tabular}{|c|c|c|}
\hline 0000012043 & 1003004020 & 0012040003 \\
\hline 0002040013 & 1004023000 & 2040010003 \\
\hline 0012040030 & 0304021000 & 0012040030 \\
\hline 0010032040 & 0301004020 & 2040010030 \\
\hline 0012040300 & 0034021000 & 0012040300 \\
\hline 0010302040 & 0031004020 & 2040010300 \\
\hline 0012043000 & 0004021003 & 0012043000 \\
\hline 0013002040 & 0001004023 & 2040013000 \\
\hline 0000012304 & 0321004000 & 0012300004 \\
\hline 0002300014 & 0324001000 & 2300010004 \\
\hline 0012300040 & 0321000400 & 0012300040 \\
\hline 0010042300 & 0320401000 & 2300010040 \\
\hline 0012300400 & 0040321000 & 0012300400 \\
\hline 0010402300 & 0041000320 & 2300010400 \\
\hline 0012304000 & 0000321004 & 0012304000 \\
\hline 0014002300 & 0001000324 & 2300014000 \\
\hline 0000012340 & 0004321000 & 0012340000 \\
\hline 0002340010 & 0001004320 & 2340010000 \\
\hline 0000012403 & 0421003000 & 0012400003 \\
\hline 0002400013 & 0423001000 & 2400010003 \\
\hline 0012400030 & 0300421000 & 0012400030 \\
\hline 0010032400 & 0301000420 & 2400010030 \\
\hline 0012400300 & 0030421000 & 0012400300 \\
\hline 0010302400 & 0031000420 & 2400010300 \\
\hline 0012403000 & 0000421003 & 0012403000 \\
\hline 0013002400 & 0001000423 & 2400013000 \\
\hline 0000012430 & 0003421000 & 0012430000 \\
\hline 0002430010 & 0001003420 & 2430010000 \\
\hline 0013000024 & 0031004200 & 0013000024 \\
\hline 0010023004 & 0034201000 & 3000010024 \\
\hline 0013000042 & 0031002400 & 0013000042 \\
\hline 0010043002 & 0032401000 & 3000010042 \\
\hline 0013000204 & 0031004020 & 0013000204 \\
\hline 0010203004 & 0034021000 & 3000010204 \\
\hline 0013000240 & 0031000420 & 0013000240 \\
\hline 0010243000 & 0030421000 & 3000010240 \\
\hline 0013000402 & 0031002040 & 0013000402 \\
\hline 0010403002 & 0032041000 & 3000010402 \\
\hline 0013000420 & 0031000240 & 0013000420 \\
\hline 0010423000 & 0030241000 & 3000010420 \\
\hline 0013002004 & 0031004002 & 0013002004 \\
\hline 0012003004 & 0034001002 & 3000012004 \\
\hline 0013002040 & 0031000402 & 0013002040 \\
\hline 0012043000 & 0030401002 & 3000012040 \\
\hline 0013002400 & 0031000042 & 0013002400 \\
\hline 0012403000 & 0030041002 & 3000012400 \\
\hline 0013004002 & 0031002004 & 0013004002 \\
\hline 0014003002 & 0032001004 & 3000014002 \\
\hline 0013004020 & 0031000204 & 0013004020 \\
\hline 0014023000 & 0030201004 & 3000014020 \\
\hline
\end{tabular}




\begin{tabular}{|c|c|c|}
\hline 0013004200 & 0020031004 & 0013004200 \\
\hline 0014203000 & 0021000034 & 3000014200 \\
\hline 0000013024 & 1004002030 & 0013020004 \\
\hline 0003020014 & 1002034000 & 3020010004 \\
\hline 0013020040 & 0402031000 & 0013020040 \\
\hline 0010043020 & 0401002030 & 3020010040 \\
\hline 0013020400 & 0042031000 & 0013020400 \\
\hline 0010403020 & 0041002030 & 3020010400 \\
\hline 0013024000 & 0002031004 & 0013024000 \\
\hline 0014003020 & 0001002034 & 3020014000 \\
\hline 0000013042 & 1002004030 & 0013040002 \\
\hline 0003040012 & 1004032000 & 3040010002 \\
\hline 0013040020 & 0204031000 & 0013040020 \\
\hline 0010023040 & 0201004030 & 3040010020 \\
\hline 0013040200 & 0024031000 & 0013040200 \\
\hline 0010203040 & 0021004030 & 3040010200 \\
\hline 0013042000 & 0004031002 & 0013042000 \\
\hline 0012003040 & 0001004032 & 3040012000 \\
\hline 0000013204 & 0231004000 & 0013200004 \\
\hline 0003200014 & 0234001000 & 3200010004 \\
\hline 0013200040 & 0231000400 & 0013200040 \\
\hline 0010043200 & 0230401000 & 3200010040 \\
\hline 0013200400 & 0040231000 & 0013200400 \\
\hline 0010403200 & 0041000230 & 3200010400 \\
\hline 0013204000 & 0000231004 & 0013204000 \\
\hline 0014003200 & 0001000234 & 3200014000 \\
\hline 0000013240 & 0004231000 & 0013240000 \\
\hline 0003240010 & 0001004230 & 3240010000 \\
\hline 0000013402 & 0431002000 & 0013400002 \\
\hline 0003400012 & 0432001000 & 3400010002 \\
\hline 0013400020 & 0200431000 & 0013400020 \\
\hline 0010023400 & 0201000430 & 3400010020 \\
\hline 0013400200 & 0020431000 & 0013400200 \\
\hline 0010203400 & 0021000430 & 3400010200 \\
\hline 0013402000 & 0000431002 & 0013402000 \\
\hline 0012003400 & 0001000432 & 3400012000 \\
\hline 0000013420 & 0002431000 & 0013420000 \\
\hline 0003420010 & 0001002430 & 3420010000 \\
\hline 0014000023 & 0041003200 & 0014000023 \\
\hline 0010024003 & 0043201000 & 4000010023 \\
\hline 0014000032 & 0041002300 & 0014000032 \\
\hline 0010034002 & 0042301000 & 4000010032 \\
\hline 0014000203 & 0041003020 & 0014000203 \\
\hline 0010204003 & 0043021000 & 4000010203 \\
\hline 0014000230 & 0041000320 & 0014000230 \\
\hline 0010234000 & 0040321000 & 4000010230 \\
\hline 0014000302 & 0041002030 & 0014000302 \\
\hline 0010304002 & 0042031000 & 4000010302 \\
\hline 0014000320 & 0041000230 & 0014000320 \\
\hline 0010324000 & 0040231000 & 4000010320 \\
\hline
\end{tabular}




\begin{tabular}{|c|c|c|}
\hline 0014002003 & 0041003002 & 0014002003 \\
\hline 0012004003 & 0043001002 & 4000012003 \\
\hline 0014002030 & 0041000302 & 0014002030 \\
\hline 0012034000 & 0040301002 & 4000012030 \\
\hline 0014002300 & 0030041002 & 0014002300 \\
\hline 0012304000 & 0031000042 & 4000012300 \\
\hline 0014003002 & 0041002003 & 0014003002 \\
\hline 0013004002 & 0042001003 & 4000013002 \\
\hline 0014003020 & 0041000203 & 0014003020 \\
\hline 0013024000 & 0040201003 & 4000013020 \\
\hline 0014003200 & 0020041003 & 0014003200 \\
\hline 0013204000 & 0021000043 & 4000013200 \\
\hline 0000014023 & 1003002040 & 0014020003 \\
\hline 0004020013 & 1002043000 & 4020010003 \\
\hline 0014020030 & 0302041000 & 0014020030 \\
\hline 0010034020 & 0301002040 & 4020010030 \\
\hline 0014020300 & 0032041000 & 0014020300 \\
\hline 0010304020 & 0031002040 & 4020010300 \\
\hline 0014023000 & 0002041003 & 0014023000 \\
\hline 0013004020 & 0001002043 & 4020013000 \\
\hline 0000014032 & 1002003040 & 0014030002 \\
\hline 0004030012 & 1003042000 & 4030010002 \\
\hline 0014030020 & 0203041000 & 0014030020 \\
\hline 0010024030 & 0201003040 & 4030010020 \\
\hline 0014030200 & 0023041000 & 0014030200 \\
\hline 0010204030 & 0021003040 & 4030010200 \\
\hline 0014032000 & 0003041002 & 0014032000 \\
\hline 0012004030 & 0001003042 & 4030012000 \\
\hline 0000014203 & 0241003000 & 0014200003 \\
\hline 0004200013 & 0243001000 & 4200010003 \\
\hline 0014200030 & 0241000300 & 0014200030 \\
\hline 0010034200 & 0240301000 & 4200010030 \\
\hline 0014200300 & 0030241000 & 0014200300 \\
\hline 0010304200 & 0031000240 & 4200010300 \\
\hline 0014203000 & 0000241003 & 0014203000 \\
\hline 0013004200 & 0001000243 & 4200013000 \\
\hline 0000014230 & 0003241000 & 0014230000 \\
\hline 0004230010 & 0001003240 & 4230010000 \\
\hline 0000014302 & 0341002000 & 0014300002 \\
\hline 0004300012 & 0342001000 & 4300010002 \\
\hline 0014300020 & 0200341000 & 0014300020 \\
\hline 0010024300 & 0201000340 & 4300010020 \\
\hline 0014300200 & 0020341000 & 0014300200 \\
\hline 0010204300 & 0021000340 & 4300010200 \\
\hline 0014302000 & 0000341002 & 0014302000 \\
\hline 0012004300 & 0001000342 & 4300012000 \\
\hline 0000014320 & 0002341000 & 0014320000 \\
\hline 0004320010 & 0001002340 & 4320010000 \\
\hline 0010020034 & 2004103000 & 0020030014 \\
\hline 0010030024 & 2003004100 & 0030020014 \\
\hline
\end{tabular}




\begin{tabular}{|c|c|c|}
\hline 0020030041 & 1403002000 & 0020030041 \\
\hline 0020040031 & 1402003000 & 0030020041 \\
\hline 0020030104 & 2004013000 & 0020030104 \\
\hline 0020100034 & 2003004010 & 0030020104 \\
\hline 0020030140 & 0413002000 & 0020030140 \\
\hline 0020140030 & 0412003000 & 0030020140 \\
\hline 0020030401 & 1043002000 & 0020030401 \\
\hline 0020400031 & 1042003000 & 0030020401 \\
\hline 0020030410 & 0143002000 & 0020030410 \\
\hline 0020410030 & 0142003000 & 0030020410 \\
\hline 0020031004 & 2004003001 & 0020031004 \\
\hline 0021000034 & 2003004001 & 0030021004 \\
\hline 0020031040 & 0403002001 & 0020031040 \\
\hline 0021040030 & 0402003001 & 0030021040 \\
\hline 0020031400 & 0043002001 & 0020031400 \\
\hline 0021400030 & 0042003001 & 0030021400 \\
\hline 0020034001 & 1003002004 & 0020034001 \\
\hline 0024000031 & 1002003004 & 0030024001 \\
\hline 0020034010 & 0103002004 & 0020034010 \\
\hline 0024010030 & 0102003004 & 0030024010 \\
\hline 0020034100 & 0013002004 & 0020034100 \\
\hline 0024100030 & 0012003004 & 0030024100 \\
\hline 0010020043 & 2003104000 & 0020040013 \\
\hline 0010040023 & 2004003100 & 0040020013 \\
\hline 0020040031 & 1304002000 & 0020040031 \\
\hline 0020030041 & 1302004000 & 0040020031 \\
\hline 0020040103 & 2003014000 & 0020040103 \\
\hline 0020100043 & 2004003010 & 0040020103 \\
\hline 0020040130 & 0314002000 & 0020040130 \\
\hline 0020130040 & 0312004000 & 0040020130 \\
\hline 0020040301 & 1034002000 & 0020040301 \\
\hline 0020300041 & 1032004000 & 0040020301 \\
\hline 0020040310 & 0134002000 & 0020040310 \\
\hline 0020310040 & 0132004000 & 0040020310 \\
\hline 0020041003 & 2003004001 & 0020041003 \\
\hline 0021000043 & 2004003001 & 0040021003 \\
\hline 0020041030 & 0304002001 & 0020041030 \\
\hline 0021030040 & 0302004001 & 0040021030 \\
\hline 0020041300 & 0034002001 & 0020041300 \\
\hline 0021300040 & 0032004001 & 0040021300 \\
\hline 0020043001 & 1004002003 & 0020043001 \\
\hline 0023000041 & 1002004003 & 0040023001 \\
\hline 0020043010 & 0104002003 & 0020043010 \\
\hline 0023010040 & 0102004003 & 0040023010 \\
\hline 0020043100 & 0014002003 & 0020043100 \\
\hline 0023100040 & 0012004003 & 0040023100 \\
\hline 0020100034 & 0102004300 & 0020100034 \\
\hline 0020030104 & 0104302000 & 0100020034 \\
\hline 0020100043 & 0102003400 & 0020100043 \\
\hline 0020040103 & 0103402000 & 0100020043 \\
\hline
\end{tabular}




\begin{tabular}{|c|c|c|}
\hline 0020100304 & 0102004030 & 0020100304 \\
\hline 0020300104 & 0104032000 & 0100020304 \\
\hline 0020100340 & 0102000430 & 0020100340 \\
\hline 0020340100 & 0100432000 & 0100020340 \\
\hline 0020100403 & 0102003040 & 0020100403 \\
\hline 0020400103 & 0103042000 & 0100020403 \\
\hline 0020100430 & 0102000340 & 0020100430 \\
\hline 0020430100 & 0100342000 & 0100020430 \\
\hline 0020103004 & 0102004003 & 0020103004 \\
\hline 0023000104 & 0104002003 & 0100023004 \\
\hline 0020103040 & 0102000403 & 0020103040 \\
\hline 0023040100 & 0100402003 & 0100023040 \\
\hline 0020103400 & 0040102003 & 0020103400 \\
\hline 0023400100 & 0042000103 & 0100023400 \\
\hline 0020104003 & 0102003004 & 0020104003 \\
\hline 0024000103 & 0103002004 & 0100024003 \\
\hline 0020104030 & 0102000304 & 0020104030 \\
\hline 0024030100 & 0100302004 & 0100024030 \\
\hline 0020104300 & 0030102004 & 0020104300 \\
\hline 0024300100 & 0032000104 & 0100024300 \\
\hline 0000020134 & 2004003100 & 0020130004 \\
\hline 0000130024 & 2003104000 & 0130020004 \\
\hline 0020130040 & 0403102000 & 0020130040 \\
\hline 0020040130 & 0402003100 & 0130020040 \\
\hline 0020130400 & 0043102000 & 0020130400 \\
\hline 0020400130 & 0042003100 & 0130020400 \\
\hline 0020134000 & 0003102004 & 0020134000 \\
\hline 0024000130 & 0002003104 & 0130024000 \\
\hline 0000020143 & 2003004100 & 0020140003 \\
\hline 0000140023 & 2004103000 & 0140020003 \\
\hline 0020140030 & 0304102000 & 0020140030 \\
\hline 0020030140 & 0302004100 & 0140020030 \\
\hline 0020140300 & 0034102000 & 0020140300 \\
\hline 0020300140 & 0032004100 & 0140020300 \\
\hline 0020143000 & 0004102003 & 0020143000 \\
\hline 0023000140 & 0002004103 & 0140023000 \\
\hline 0010020304 & 0302004100 & 0020300014 \\
\hline 0010300024 & 0304102000 & 0300020014 \\
\hline 0020300041 & 0302001400 & 0020300041 \\
\hline 0020040301 & 0301402000 & 0300020041 \\
\hline 0020300104 & 0302004010 & 0020300104 \\
\hline 0020100304 & 0304012000 & 0300020104 \\
\hline 0020300140 & 0302000410 & 0020300140 \\
\hline 0020140300 & 0300412000 & 0300020140 \\
\hline 0020300401 & 0302001040 & 0020300401 \\
\hline 0020400301 & 0301042000 & 0300020401 \\
\hline 0020300410 & 0140302000 & 0020300410 \\
\hline 0020410300 & 0142000300 & 0300020410 \\
\hline 0020301004 & 0302004001 & 0020301004 \\
\hline 0021000304 & 0304002001 & 0300021004 \\
\hline
\end{tabular}




\begin{tabular}{|c|c|c|}
\hline 0020301040 & 0302000401 & 0020301040 \\
\hline 0021040300 & 0300402001 & 0300021040 \\
\hline 0020301400 & 0040302001 & 0020301400 \\
\hline 0021400300 & 0042000301 & 0300021400 \\
\hline 0020304001 & 0302001004 & 0020304001 \\
\hline 0024000301 & 0301002004 & 0300024001 \\
\hline 0020304010 & 0100302004 & 0020304010 \\
\hline 0024010300 & 0102000304 & 0300024010 \\
\hline 0020304100 & 0010302004 & 0020304100 \\
\hline 0024100300 & 0012000304 & 0300024100 \\
\hline 0000020314 & 1302004000 & 0020310004 \\
\hline 0000310024 & 1304002000 & 0310020004 \\
\hline 0020310040 & 0401302000 & 0020310040 \\
\hline 0020040310 & 0402001300 & 0310020040 \\
\hline 0020310400 & 0041302000 & 0020310400 \\
\hline 0020400310 & 0042001300 & 0310020400 \\
\hline 0020314000 & 0001302004 & 0020314000 \\
\hline 0024000310 & 0002001304 & 0310024000 \\
\hline 0000020341 & 1004302000 & 0020340001 \\
\hline 0000340021 & 1002004300 & 0340020001 \\
\hline 0010020340 & 0104302000 & 0020340010 \\
\hline 0010340020 & 0102004300 & 0340020010 \\
\hline 0020340100 & 0014302000 & 0020340100 \\
\hline 0020100340 & 0012004300 & 0340020100 \\
\hline 0020341000 & 0004302001 & 0020341000 \\
\hline 0021000340 & 0002004301 & 0340021000 \\
\hline 0010020403 & 0402003100 & 0020400013 \\
\hline 0010400023 & 0403102000 & 0400020013 \\
\hline 0020400031 & 0402001300 & 0020400031 \\
\hline 0020030401 & 0401302000 & 0400020031 \\
\hline 0020400103 & 0402003010 & 0020400103 \\
\hline 0020100403 & 0403012000 & 0400020103 \\
\hline 0020400130 & 0310402000 & 0020400130 \\
\hline 0020130400 & 0312000400 & 0400020130 \\
\hline 0020400301 & 0402001030 & 0020400301 \\
\hline 0020300401 & 0401032000 & 0400020301 \\
\hline 0020400310 & 0130402000 & 0020400310 \\
\hline 0020310400 & 0132000400 & 0400020310 \\
\hline 0020401003 & 0402003001 & 0020401003 \\
\hline 0021000403 & 0403002001 & 0400021003 \\
\hline 0020401030 & 0300402001 & 0020401030 \\
\hline 0021030400 & 0302000401 & 0400021030 \\
\hline 0020401300 & 0030402001 & 0020401300 \\
\hline 0021300400 & 0032000401 & 0400021300 \\
\hline 0020403001 & 0402001003 & 0020403001 \\
\hline 0023000401 & 0401002003 & 0400023001 \\
\hline 0020403010 & 0100402003 & 0020403010 \\
\hline 0023010400 & 0102000403 & 0400023010 \\
\hline 0020403100 & 0010402003 & 0020403100 \\
\hline 0023100400 & 0012000403 & 0400023100 \\
\hline
\end{tabular}




\begin{tabular}{|c|c|c|}
\hline 0000020413 & 1402003000 & 0020410003 \\
\hline 0000410023 & 1403002000 & 0410020003 \\
\hline 0020410030 & 0301402000 & 0020410030 \\
\hline 0020030410 & 0302001400 & 0410020030 \\
\hline 0020410300 & 0031402000 & 0020410300 \\
\hline 0020300410 & 0032001400 & 0410020300 \\
\hline 0020413000 & 0001402003 & 0020413000 \\
\hline 0023000410 & 0002001403 & 0410023000 \\
\hline 0000020431 & 1003402000 & 0020430001 \\
\hline 0000430021 & 1002003400 & 0430020001 \\
\hline 0010020430 & 0103402000 & 0020430010 \\
\hline 0010430020 & 0102003400 & 0430020010 \\
\hline 0020430100 & 0013402000 & 0020430100 \\
\hline 0020100430 & 0012003400 & 0430020100 \\
\hline 0020431000 & 0003402001 & 0020431000 \\
\hline 0021000430 & 0002003401 & 0430021000 \\
\hline 0000021034 & 2004003010 & 0021030004 \\
\hline 0001030024 & 2003014000 & 1030020004 \\
\hline 0021030040 & 0403012000 & 0021030040 \\
\hline 0020041030 & 0402003010 & 1030020040 \\
\hline 0021030400 & 0043012000 & 0021030400 \\
\hline 0020401030 & 0042003010 & 1030020400 \\
\hline 0021034000 & 0003012004 & 0021034000 \\
\hline 0024001030 & 0002003014 & 1030024000 \\
\hline 0000021043 & 2003004010 & 0021040003 \\
\hline 0001040023 & 2004013000 & 1040020003 \\
\hline 0021040030 & 0304012000 & 0021040030 \\
\hline 0020031040 & 0302004010 & 1040020030 \\
\hline 0021040300 & 0034012000 & 0021040300 \\
\hline 0020301040 & 0032004010 & 1040020300 \\
\hline 0021043000 & 0004012003 & 0021043000 \\
\hline 0023001040 & 0002004013 & 1040023000 \\
\hline 0000021304 & 0312004000 & 0021300004 \\
\hline 0001300024 & 0314002000 & 1300020004 \\
\hline 0021300040 & 0312000400 & 0021300040 \\
\hline 0020041300 & 0310402000 & 1300020040 \\
\hline 0021300400 & 0040312000 & 0021300400 \\
\hline 0020401300 & 0042000310 & 1300020400 \\
\hline 0021304000 & 0000312004 & 0021304000 \\
\hline 0024001300 & 0002000314 & 1300024000 \\
\hline 0000021340 & 0004312000 & 0021340000 \\
\hline 0001340020 & 0002004310 & 1340020000 \\
\hline 0000021403 & 0412003000 & 0021400003 \\
\hline 0001400023 & 0413002000 & 1400020003 \\
\hline 0021400030 & 0300412000 & 0021400030 \\
\hline 0020031400 & 0302000410 & 1400020030 \\
\hline 0021400300 & 0030412000 & 0021400300 \\
\hline 0020301400 & 0032000410 & 1400020300 \\
\hline 0021403000 & 0000412003 & 0021403000 \\
\hline 0023001400 & 0002000413 & 1400023000 \\
\hline
\end{tabular}




\begin{tabular}{|c|c|c|}
\hline 0000021430 & 0003412000 & 0021430000 \\
\hline 0001430020 & 0002003410 & 1430020000 \\
\hline 0010023004 & 0032004100 & 0023000014 \\
\hline 0013000024 & 0034102000 & 3000020014 \\
\hline 0023000041 & 0032001400 & 0023000041 \\
\hline 0020043001 & 0031402000 & 3000020041 \\
\hline 0023000104 & 0032004010 & 0023000104 \\
\hline 0020103004 & 0034012000 & 3000020104 \\
\hline 0023000140 & 0032000410 & 0023000140 \\
\hline 0020143000 & 0030412000 & 3000020140 \\
\hline 0023000401 & 0032001040 & 0023000401 \\
\hline 0020403001 & 0031042000 & 3000020401 \\
\hline 0023000410 & 0032000140 & 0023000410 \\
\hline 0020413000 & 0030142000 & 3000020410 \\
\hline 0023001004 & 0032004001 & 0023001004 \\
\hline 0021003004 & 0034002001 & 3000021004 \\
\hline 0023001040 & 0032000401 & 0023001040 \\
\hline 0021043000 & 0030402001 & 3000021040 \\
\hline 0023001400 & 0032000041 & 0023001400 \\
\hline 0021403000 & 0030042001 & 3000021400 \\
\hline 0023004001 & 0032001004 & 0023004001 \\
\hline 0024003001 & 0031002004 & 3000024001 \\
\hline 0023004010 & 0032000104 & 0023004010 \\
\hline 0024013000 & 0030102004 & 3000024010 \\
\hline 0023004100 & 0010032004 & 0023004100 \\
\hline 0024103000 & 0012000034 & 3000024100 \\
\hline 0000023014 & 1032004000 & 0023010004 \\
\hline 0003010024 & 1034002000 & 3010020004 \\
\hline 0023010040 & 0401032000 & 0023010040 \\
\hline 0020043010 & 0402001030 & 3010020040 \\
\hline 0023010400 & 0041032000 & 0023010400 \\
\hline 0020403010 & 0042001030 & 3010020400 \\
\hline 0023014000 & 0001032004 & 0023014000 \\
\hline 0024003010 & 0002001034 & 3010024000 \\
\hline 0000023041 & 1004032000 & 0023040001 \\
\hline 0003040021 & 1002004030 & 3040020001 \\
\hline 0010023040 & 0104032000 & 0023040010 \\
\hline 0013040020 & 0102004030 & 3040020010 \\
\hline 0023040100 & 0014032000 & 0023040100 \\
\hline 0020103040 & 0012004030 & 3040020100 \\
\hline 0023041000 & 0004032001 & 0023041000 \\
\hline 0021003040 & 0002004031 & 3040021000 \\
\hline 0000023104 & 0132004000 & 0023100004 \\
\hline 0003100024 & 0134002000 & 3100020004 \\
\hline 0023100040 & 0132000400 & 0023100040 \\
\hline 0020043100 & 0130402000 & 3100020040 \\
\hline 0023100400 & 0040132000 & 0023100400 \\
\hline 0020403100 & 0042000130 & 3100020400 \\
\hline 0023104000 & 0000132004 & 0023104000 \\
\hline 0024003100 & 0002000134 & 3100024000 \\
\hline
\end{tabular}




\begin{tabular}{|c|c|c|}
\hline 0000023140 & 0004132000 & 0023140000 \\
\hline 0003140020 & 0002004130 & 3140020000 \\
\hline 0000023401 & 0432001000 & 0023400001 \\
\hline 0003400021 & 0431002000 & 3400020001 \\
\hline 0010023400 & 0100432000 & 0023400010 \\
\hline 0013400020 & 0102000430 & 3400020010 \\
\hline 0023400100 & 0010432000 & 0023400100 \\
\hline 0020103400 & 0012000430 & 3400020100 \\
\hline 0023401000 & 0000432001 & 0023401000 \\
\hline 0021003400 & 0002000431 & 3400021000 \\
\hline 0000023410 & 0001432000 & 0023410000 \\
\hline 0003410020 & 0002001430 & 3410020000 \\
\hline 0010024003 & 0042003100 & 0024000013 \\
\hline 0014000023 & 0043102000 & 4000020013 \\
\hline 0024000031 & 0042001300 & 0024000031 \\
\hline 0020034001 & 0041302000 & 4000020031 \\
\hline 0024000103 & 0042003010 & 0024000103 \\
\hline 0020104003 & 0043012000 & 4000020103 \\
\hline 0024000130 & 0042000310 & 0024000130 \\
\hline 0020134000 & 0040312000 & 4000020130 \\
\hline 0024000301 & 0042001030 & 0024000301 \\
\hline 0020304001 & 0041032000 & 4000020301 \\
\hline 0024000310 & 0042000130 & 0024000310 \\
\hline 0020314000 & 0040132000 & 4000020310 \\
\hline 0024001003 & 0042003001 & 0024001003 \\
\hline 0021004003 & 0043002001 & 4000021003 \\
\hline 0024001030 & 0042000301 & 0024001030 \\
\hline 0021034000 & 0040302001 & 4000021030 \\
\hline 0024001300 & 0030042001 & 0024001300 \\
\hline 0021304000 & 0032000041 & 4000021300 \\
\hline 0024003001 & 0042001003 & 0024003001 \\
\hline 0023004001 & 0041002003 & 4000023001 \\
\hline 0024003010 & 0042000103 & 0024003010 \\
\hline 0023014000 & 0040102003 & 4000023010 \\
\hline 0024003100 & 0010042003 & 0024003100 \\
\hline 0023104000 & 0012000043 & 4000023100 \\
\hline 0000024013 & 1042003000 & 0024010003 \\
\hline 0004010023 & 1043002000 & 4010020003 \\
\hline 0024010030 & 0301042000 & 0024010030 \\
\hline 0020034010 & 0302001040 & 4010020030 \\
\hline 0024010300 & 0031042000 & 0024010300 \\
\hline 0020304010 & 0032001040 & 4010020300 \\
\hline 0024013000 & 0001042003 & 0024013000 \\
\hline 0023004010 & 0002001043 & 4010023000 \\
\hline 0000024031 & 1003042000 & 0024030001 \\
\hline 0004030021 & 1002003040 & 4030020001 \\
\hline 0010024030 & 0103042000 & 0024030010 \\
\hline 0014030020 & 0102003040 & 4030020010 \\
\hline 0024030100 & 0013042000 & 0024030100 \\
\hline 0020104030 & 0012003040 & 4030020100 \\
\hline
\end{tabular}




\begin{tabular}{|c|c|c|}
\hline 0024031000 & 0003042001 & 0024031000 \\
\hline 0021004030 & 0002003041 & 4030021000 \\
\hline 0000024103 & 0142003000 & 0024100003 \\
\hline 0004100023 & 0143002000 & 4100020003 \\
\hline 0024100030 & 0142000300 & 0024100030 \\
\hline 0020034100 & 0140302000 & 4100020030 \\
\hline 0024100300 & 0030142000 & 0024100300 \\
\hline 0020304100 & 0032000140 & 4100020300 \\
\hline 0024103000 & 0000142003 & 0024103000 \\
\hline 0023004100 & 0002000143 & 4100023000 \\
\hline 0000024130 & 0003142000 & 0024130000 \\
\hline 0004130020 & 0002003140 & 4130020000 \\
\hline 0000024301 & 0342001000 & 0024300001 \\
\hline 0004300021 & 0341002000 & 4300020001 \\
\hline 0010024300 & 0100342000 & 0024300010 \\
\hline 0014300020 & 0102000340 & 4300020010 \\
\hline 0024300100 & 0010342000 & 0024300100 \\
\hline 0020104300 & 0012000340 & 4300020100 \\
\hline 0024301000 & 0000342001 & 0024301000 \\
\hline 0021004300 & 0002000341 & 4300021000 \\
\hline 0000024310 & 0001342000 & 0024310000 \\
\hline 0004310020 & 0002001340 & 4310020000 \\
\hline 0010030042 & 2104003000 & 0030040012 \\
\hline 0010040032 & 2103004000 & 0040030012 \\
\hline 0020030041 & 1204003000 & 0030040021 \\
\hline 0020040031 & 1203004000 & 0040030021 \\
\hline 0030040102 & 2014003000 & 0030040102 \\
\hline 0030100042 & 2013004000 & 0040030102 \\
\hline 0030040120 & 0214003000 & 0030040120 \\
\hline 0030120040 & 0213004000 & 0040030120 \\
\hline 0030040201 & 1024003000 & 0030040201 \\
\hline 0030200041 & 1023004000 & 0040030201 \\
\hline 0030040210 & 0124003000 & 0030040210 \\
\hline 0030210040 & 0123004000 & 0040030210 \\
\hline 0030041002 & 2004003001 & 0030041002 \\
\hline 0031000042 & 2003004001 & 0040031002 \\
\hline 0030041020 & 0204003001 & 0030041020 \\
\hline 0031020040 & 0203004001 & 0040031020 \\
\hline 0030041200 & 0024003001 & 0030041200 \\
\hline 0031200040 & 0023004001 & 0040031200 \\
\hline 0030042001 & 1004003002 & 0030042001 \\
\hline 0032000041 & 1003004002 & 0040032001 \\
\hline 0030042010 & 0104003002 & 0030042010 \\
\hline 0032010040 & 0103004002 & 0040032010 \\
\hline 0030042100 & 0014003002 & 0030042100 \\
\hline 0032100040 & 0013004002 & 0040032100 \\
\hline 0020030104 & 0103004200 & 0030100024 \\
\hline 0020100034 & 0104203000 & 0100030024 \\
\hline 0030100042 & 0103002400 & 0030100042 \\
\hline 0030040102 & 0102403000 & 0100030042 \\
\hline
\end{tabular}




\begin{tabular}{|c|c|c|}
\hline 0030100204 & 0103004020 & 0030100204 \\
\hline 0030200104 & 0104023000 & 0100030204 \\
\hline 0030100240 & 0103000420 & 0030100240 \\
\hline 0030240100 & 0100423000 & 0100030240 \\
\hline 0030100402 & 0103002040 & 0030100402 \\
\hline 0030400102 & 0102043000 & 0100030402 \\
\hline 0030100420 & 0103000240 & 0030100420 \\
\hline 0030420100 & 0100243000 & 0100030420 \\
\hline 0030102004 & 0103004002 & 0030102004 \\
\hline 0032000104 & 0104003002 & 0100032004 \\
\hline 0030102040 & 0103000402 & 0030102040 \\
\hline 0032040100 & 0100403002 & 0100032040 \\
\hline 0030102400 & 0040103002 & 0030102400 \\
\hline 0032400100 & 0043000102 & 0100032400 \\
\hline 0030104002 & 0103002004 & 0030104002 \\
\hline 0034000102 & 0102003004 & 0100034002 \\
\hline 0030104020 & 0103000204 & 0030104020 \\
\hline 0034020100 & 0100203004 & 0100034020 \\
\hline 0030104200 & 0020103004 & 0030104200 \\
\hline 0034200100 & 0023000104 & 0100034200 \\
\hline 0000030124 & 2103004000 & 0030120004 \\
\hline 0000120034 & 2104003000 & 0120030004 \\
\hline 0030120040 & 0402103000 & 0030120040 \\
\hline 0030040120 & 0403002100 & 0120030040 \\
\hline 0030120400 & 0042103000 & 0030120400 \\
\hline 0030400120 & 0043002100 & 0120030400 \\
\hline 0030124000 & 0002103004 & 0030124000 \\
\hline 0034000120 & 0003002104 & 0120034000 \\
\hline 0000030142 & 2004103000 & 0030140002 \\
\hline 0000140032 & 2003004100 & 0140030002 \\
\hline 0020030140 & 0204103000 & 0030140020 \\
\hline 0020140030 & 0203004100 & 0140030020 \\
\hline 0030140200 & 0024103000 & 0030140200 \\
\hline 0030200140 & 0023004100 & 0140030200 \\
\hline 0030142000 & 0004103002 & 0030142000 \\
\hline 0032000140 & 0003004102 & 0140032000 \\
\hline 0010030204 & 0203004100 & 0030200014 \\
\hline 0010200034 & 0204103000 & 0200030014 \\
\hline 0030200041 & 0203001400 & 0030200041 \\
\hline 0030040201 & 0201403000 & 0200030041 \\
\hline 0030200104 & 0203004010 & 0030200104 \\
\hline 0030100204 & 0204013000 & 0200030104 \\
\hline 0030200140 & 0203000410 & 0030200140 \\
\hline 0030140200 & 0200413000 & 0200030140 \\
\hline 0030200401 & 0203001040 & 0030200401 \\
\hline 0030400201 & 0201043000 & 0200030401 \\
\hline 0030200410 & 0140203000 & 0030200410 \\
\hline 0030410200 & 0143000200 & 0200030410 \\
\hline 0030201004 & 0203004001 & 0030201004 \\
\hline 0031000204 & 0204003001 & 0200031004 \\
\hline
\end{tabular}




\begin{tabular}{|c|c|c|}
\hline 0030201040 & 0203000401 & 0030201040 \\
\hline 0031040200 & 0200403001 & 0200031040 \\
\hline 0030201400 & 0040203001 & 0030201400 \\
\hline 0031400200 & 0043000201 & 0200031400 \\
\hline 0030204001 & 0203001004 & 0030204001 \\
\hline 0034000201 & 0201003004 & 0200034001 \\
\hline 0030204010 & 0100203004 & 0030204010 \\
\hline 0034010200 & 0103000204 & 0200034010 \\
\hline 0030204100 & 0010203004 & 0030204100 \\
\hline 0034100200 & 0013000204 & 0200034100 \\
\hline 0000030214 & 1203004000 & 0030210004 \\
\hline 0000210034 & 1204003000 & 0210030004 \\
\hline 0030210040 & 0401203000 & 0030210040 \\
\hline 0030040210 & 0403001200 & 0210030040 \\
\hline 0030210400 & 0041203000 & 0030210400 \\
\hline 0030400210 & 0043001200 & 0210030400 \\
\hline 0030214000 & 0001203004 & 0030214000 \\
\hline 0034000210 & 0003001204 & 0210034000 \\
\hline 0000030241 & 1004203000 & 0030240001 \\
\hline 0000240031 & 1003004200 & 0240030001 \\
\hline 0010030240 & 0104203000 & 0030240010 \\
\hline 0010240030 & 0103004200 & 0240030010 \\
\hline 0030240100 & 0014203000 & 0030240100 \\
\hline 0030100240 & 0013004200 & 0240030100 \\
\hline 0030241000 & 0004203001 & 0030241000 \\
\hline 0031000240 & 0003004201 & 0240031000 \\
\hline 0010030402 & 0403002100 & 0030400012 \\
\hline 0010400032 & 0402103000 & 0400030012 \\
\hline 0020030401 & 0403001200 & 0030400021 \\
\hline 0020400031 & 0401203000 & 0400030021 \\
\hline 0030400102 & 0403002010 & 0030400102 \\
\hline 0030100402 & 0402013000 & 0400030102 \\
\hline 0030400120 & 0210403000 & 0030400120 \\
\hline 0030120400 & 0213000400 & 0400030120 \\
\hline 0030400201 & 0403001020 & 0030400201 \\
\hline 0030200401 & 0401023000 & 0400030201 \\
\hline 0030400210 & 0120403000 & 0030400210 \\
\hline 0030210400 & 0123000400 & 0400030210 \\
\hline 0030401002 & 0403002001 & 0030401002 \\
\hline 0031000402 & 0402003001 & 0400031002 \\
\hline 0030401020 & 0200403001 & 0030401020 \\
\hline 0031020400 & 0203000401 & 0400031020 \\
\hline 0030401200 & 0020403001 & 0030401200 \\
\hline 0031200400 & 0023000401 & 0400031200 \\
\hline 0030402001 & 0403001002 & 0030402001 \\
\hline 0032000401 & 0401003002 & 0400032001 \\
\hline 0030402010 & 0100403002 & 0030402010 \\
\hline 0032010400 & 0103000402 & 0400032010 \\
\hline 0030402100 & 0010403002 & 0030402100 \\
\hline 0032100400 & 0013000402 & 0400032100 \\
\hline
\end{tabular}




\begin{tabular}{|c|c|c|}
\hline 0000030412 & 1403002000 & 0030410002 \\
\hline 0000410032 & 1402003000 & 0410030002 \\
\hline 0020030410 & 0201403000 & 0030410020 \\
\hline 0020410030 & 0203001400 & 0410030020 \\
\hline 0030410200 & 0021403000 & 0030410200 \\
\hline 0030200410 & 0023001400 & 0410030200 \\
\hline 0030412000 & 0001403002 & 0030412000 \\
\hline 0032000410 & 0003001402 & 0410032000 \\
\hline 0000030421 & 1002403000 & 0030420001 \\
\hline 0000420031 & 1003002400 & 0420030001 \\
\hline 0010030420 & 0102403000 & 0030420010 \\
\hline 0010420030 & 0103002400 & 0420030010 \\
\hline 0030420100 & 0012403000 & 0030420100 \\
\hline 0030100420 & 0013002400 & 0420030100 \\
\hline 0030421000 & 0002403001 & 0030421000 \\
\hline 0031000420 & 0003002401 & 0420031000 \\
\hline 0000031024 & 2013004000 & 0031020004 \\
\hline 0001020034 & 2014003000 & 1020030004 \\
\hline 0031020040 & 0402013000 & 0031020040 \\
\hline 0030041020 & 0403002010 & 1020030040 \\
\hline 0031020400 & 0042013000 & 0031020400 \\
\hline 0030401020 & 0043002010 & 1020030400 \\
\hline 0031024000 & 0002013004 & 0031024000 \\
\hline 0034001020 & 0003002014 & 1020034000 \\
\hline 0000031042 & 2004013000 & 0031040002 \\
\hline 0001040032 & 2003004010 & 1040030002 \\
\hline 0020031040 & 0204013000 & 0031040020 \\
\hline 0021040030 & 0203004010 & 1040030020 \\
\hline 0031040200 & 0024013000 & 0031040200 \\
\hline 0030201040 & 0023004010 & 1040030200 \\
\hline 0031042000 & 0004013002 & 0031042000 \\
\hline 0032001040 & 0003004012 & 1040032000 \\
\hline 0000031204 & 0213004000 & 0031200004 \\
\hline 0001200034 & 0214003000 & 1200030004 \\
\hline 0031200040 & 0213000400 & 0031200040 \\
\hline 0030041200 & 0210403000 & 1200030040 \\
\hline 0031200400 & 0040213000 & 0031200400 \\
\hline 0030401200 & 0043000210 & 1200030400 \\
\hline 0031204000 & 0000213004 & 0031204000 \\
\hline 0034001200 & 0003000214 & 1200034000 \\
\hline 0000031240 & 0004213000 & 0031240000 \\
\hline 0001240030 & 0003004210 & 1240030000 \\
\hline 0000031402 & 0413002000 & 0031400002 \\
\hline 0001400032 & 0412003000 & 1400030002 \\
\hline 0020031400 & 0200413000 & 0031400020 \\
\hline 0021400030 & 0203000410 & 1400030020 \\
\hline 0031400200 & 0020413000 & 0031400200 \\
\hline 0030201400 & 0023000410 & 1400030200 \\
\hline 0031402000 & 0000413002 & 0031402000 \\
\hline 0032001400 & 0003000412 & 1400032000 \\
\hline
\end{tabular}




\begin{tabular}{|c|c|c|}
\hline 0000031420 & 0002413000 & 0031420000 \\
\hline 0001420030 & 0003002410 & 1420030000 \\
\hline 0000032014 & 1023004000 & 0032010004 \\
\hline 0002010034 & 1024003000 & 2010030004 \\
\hline 0032010040 & 0401023000 & 0032010040 \\
\hline 0030042010 & 0403001020 & 2010030040 \\
\hline 0032010400 & 0041023000 & 0032010400 \\
\hline 0030402010 & 0043001020 & 2010030400 \\
\hline 0032014000 & 0001023004 & 0032014000 \\
\hline 0034002010 & 0003001024 & 2010034000 \\
\hline 0000032041 & 1004023000 & 0032040001 \\
\hline 0002040031 & 1003004020 & 2040030001 \\
\hline 0010032040 & 0104023000 & 0032040010 \\
\hline 0012040030 & 0103004020 & 2040030010 \\
\hline 0032040100 & 0014023000 & 0032040100 \\
\hline 0030102040 & 0013004020 & 2040030100 \\
\hline 0032041000 & 0004023001 & 0032041000 \\
\hline 0031002040 & 0003004021 & 2040031000 \\
\hline 0000032104 & 0123004000 & 0032100004 \\
\hline 0002100034 & 0124003000 & 2100030004 \\
\hline 0032100040 & 0123000400 & 0032100040 \\
\hline 0030042100 & 0120403000 & 2100030040 \\
\hline 0032100400 & 0040123000 & 0032100400 \\
\hline 0030402100 & 0043000120 & 2100030400 \\
\hline 0032104000 & 0000123004 & 0032104000 \\
\hline 0034002100 & 0003000124 & 2100034000 \\
\hline 0000032140 & 0004123000 & 0032140000 \\
\hline 0002140030 & 0003004120 & 2140030000 \\
\hline 0000032401 & 0423001000 & 0032400001 \\
\hline 0002400031 & 0421003000 & 2400030001 \\
\hline 0010032400 & 0100423000 & 0032400010 \\
\hline 0012400030 & 0103000420 & 2400030010 \\
\hline 0032400100 & 0010423000 & 0032400100 \\
\hline 0030102400 & 0013000420 & 2400030100 \\
\hline 0032401000 & 0000423001 & 0032401000 \\
\hline 0031002400 & 0003000421 & 2400031000 \\
\hline 0000032410 & 0001423000 & 0032410000 \\
\hline 0002410030 & 0003001420 & 2410030000 \\
\hline 0010034002 & 0043002100 & 0034000012 \\
\hline 0014000032 & 0042103000 & 4000030012 \\
\hline 0020034001 & 0043001200 & 0034000021 \\
\hline 0024000031 & 0041203000 & 4000030021 \\
\hline 0034000102 & 0043002010 & 0034000102 \\
\hline 0030104002 & 0042013000 & 4000030102 \\
\hline 0034000120 & 0043000210 & 0034000120 \\
\hline 0030124000 & 0040213000 & 4000030120 \\
\hline 0034000201 & 0043001020 & 0034000201 \\
\hline 0030204001 & 0041023000 & 4000030201 \\
\hline 0034000210 & 0043000120 & 0034000210 \\
\hline 0030214000 & 0040123000 & 4000030210 \\
\hline
\end{tabular}




\begin{tabular}{|c|c|c|}
\hline 0034001002 & 0043002001 & 0034001002 \\
\hline 0031004002 & 0042003001 & 4000031002 \\
\hline 0034001020 & 0043000201 & 0034001020 \\
\hline 0031024000 & 0040203001 & 4000031020 \\
\hline 0034001200 & 0020043001 & 0034001200 \\
\hline 0031204000 & 0023000041 & 4000031200 \\
\hline 0034002001 & 0043001002 & 0034002001 \\
\hline 0032004001 & 0041003002 & 4000032001 \\
\hline 0034002010 & 0043000102 & 0034002010 \\
\hline 0032014000 & 0040103002 & 4000032010 \\
\hline 0034002100 & 0010043002 & 0034002100 \\
\hline 0032104000 & 0013000042 & 4000032100 \\
\hline 0000034012 & 1043002000 & 0034010002 \\
\hline 0004010032 & 1042003000 & 4010030002 \\
\hline 0020034010 & 0201043000 & 0034010020 \\
\hline 0024010030 & 0203001040 & 4010030020 \\
\hline 0034010200 & 0021043000 & 0034010200 \\
\hline 0030204010 & 0023001040 & 4010030200 \\
\hline 0034012000 & 0001043002 & 0034012000 \\
\hline 0032004010 & 0003001042 & 4010032000 \\
\hline 0000034021 & 1002043000 & 0034020001 \\
\hline 0004020031 & 1003002040 & 4020030001 \\
\hline 0010034020 & 0102043000 & 0034020010 \\
\hline 0014020030 & 0103002040 & 4020030010 \\
\hline 0034020100 & 0012043000 & 0034020100 \\
\hline 0030104020 & 0013002040 & 4020030100 \\
\hline 0034021000 & 0002043001 & 0034021000 \\
\hline 0031004020 & 0003002041 & 4020031000 \\
\hline 0000034102 & 0143002000 & 0034100002 \\
\hline 0004100032 & 0142003000 & 4100030002 \\
\hline 0020034100 & 0143000200 & 0034100020 \\
\hline 0024100030 & 0140203000 & 4100030020 \\
\hline 0034100200 & 0020143000 & 0034100200 \\
\hline 0030204100 & 0023000140 & 4100030200 \\
\hline 0034102000 & 0000143002 & 0034102000 \\
\hline 0032004100 & 0003000142 & 4100032000 \\
\hline 0000034120 & 0002143000 & 0034120000 \\
\hline 0004120030 & 0003002140 & 4120030000 \\
\hline 0000034201 & 0243001000 & 0034200001 \\
\hline 0004200031 & 0241003000 & 4200030001 \\
\hline 0010034200 & 0100243000 & 0034200010 \\
\hline 0014200030 & 0103000240 & 4200030010 \\
\hline 0034200100 & 0010243000 & 0034200100 \\
\hline 0030104200 & 0013000240 & 4200030100 \\
\hline 0034201000 & 0000243001 & 0034201000 \\
\hline 0031004200 & 0003000241 & 4200031000 \\
\hline 0000034210 & 0001243000 & 0034210000 \\
\hline 0004210030 & 0003001240 & 4210030000 \\
\hline 0020040103 & 0104003200 & 0040100023 \\
\hline 0020100043 & 0103204000 & 0100040023 \\
\hline
\end{tabular}




\begin{tabular}{|c|c|c|}
\hline 0030040102 & 0104002300 & 0040100032 \\
\hline 0030100042 & 0102304000 & 0100040032 \\
\hline 0040100203 & 0104003020 & 0040100203 \\
\hline 0040200103 & 0103024000 & 0100040203 \\
\hline 0040100230 & 0104000320 & 0040100230 \\
\hline 0040230100 & 0100324000 & 0100040230 \\
\hline 0040100302 & 0104002030 & 0040100302 \\
\hline 0040300102 & 0102034000 & 0100040302 \\
\hline 0040100320 & 0104000230 & 0040100320 \\
\hline 0040320100 & 0100234000 & 0100040320 \\
\hline 0040102003 & 0104003002 & 0040102003 \\
\hline 0042000103 & 0103004002 & 0100042003 \\
\hline 0040102030 & 0104000302 & 0040102030 \\
\hline 0042030100 & 0100304002 & 0100042030 \\
\hline 0040102300 & 0030104002 & 0040102300 \\
\hline 0042300100 & 0034000102 & 0100042300 \\
\hline 0040103002 & 0104002003 & 0040103002 \\
\hline 0043000102 & 0102004003 & 0100043002 \\
\hline 0040103020 & 0104000203 & 0040103020 \\
\hline 0043020100 & 0100204003 & 0100043020 \\
\hline 0040103200 & 0020104003 & 0040103200 \\
\hline 0043200100 & 0024000103 & 0100043200 \\
\hline 0000040123 & 2104003000 & 0040120003 \\
\hline 0000120043 & 2103004000 & 0120040003 \\
\hline 0030040120 & 0302104000 & 0040120030 \\
\hline 0030120040 & 0304002100 & 0120040030 \\
\hline 0040120300 & 0032104000 & 0040120300 \\
\hline 0040300120 & 0034002100 & 0120040300 \\
\hline 0040123000 & 0002104003 & 0040123000 \\
\hline 0043000120 & 0004002103 & 0120043000 \\
\hline 0000040132 & 2003104000 & 0040130002 \\
\hline 0000130042 & 2004003100 & 0130040002 \\
\hline 0020040130 & 0203104000 & 0040130020 \\
\hline 0020130040 & 0204003100 & 0130040020 \\
\hline 0040130200 & 0023104000 & 0040130200 \\
\hline 0040200130 & 0024003100 & 0130040200 \\
\hline 0040132000 & 0003104002 & 0040132000 \\
\hline 0042000130 & 0004003102 & 0130042000 \\
\hline 0010040203 & 0204003100 & 0040200013 \\
\hline 0010200043 & 0203104000 & 0200040013 \\
\hline 0030040201 & 0204001300 & 0040200031 \\
\hline 0030200041 & 0201304000 & 0200040031 \\
\hline 0040200103 & 0204003010 & 0040200103 \\
\hline 0040100203 & 0203014000 & 0200040103 \\
\hline 0040200130 & 0204000310 & 0040200130 \\
\hline 0040130200 & 0200314000 & 0200040130 \\
\hline 0040200301 & 0204001030 & 0040200301 \\
\hline 0040300201 & 0201034000 & 0200040301 \\
\hline 0040200310 & 0130204000 & 0040200310 \\
\hline 0040310200 & 0134000200 & 0200040310 \\
\hline
\end{tabular}




\begin{tabular}{|c|c|c|}
\hline 0040201003 & 0204003001 & 0040201003 \\
\hline 0041000203 & 0203004001 & 0200041003 \\
\hline 0040201030 & 0204000301 & 0040201030 \\
\hline 0041030200 & 0200304001 & 0200041030 \\
\hline 0040201300 & 0030204001 & 0040201300 \\
\hline 0041300200 & 0034000201 & 0200041300 \\
\hline 0040203001 & 0204001003 & 0040203001 \\
\hline 0043000201 & 0201004003 & 0200043001 \\
\hline 0040203010 & 0100204003 & 0040203010 \\
\hline 0043010200 & 0104000203 & 0200043010 \\
\hline 0040203100 & 0010204003 & 0040203100 \\
\hline 0043100200 & 0014000203 & 0200043100 \\
\hline 0000040213 & 1204003000 & 0040210003 \\
\hline 0000210043 & 1203004000 & 0210040003 \\
\hline 0030040210 & 0301204000 & 0040210030 \\
\hline 0030210040 & 0304001200 & 0210040030 \\
\hline 0040210300 & 0031204000 & 0040210300 \\
\hline 0040300210 & 0034001200 & 0210040300 \\
\hline 0040213000 & 0001204003 & 0040213000 \\
\hline 0043000210 & 0004001203 & 0210043000 \\
\hline 0000040231 & 1003204000 & 0040230001 \\
\hline 0000230041 & 1004003200 & 0230040001 \\
\hline 0010040230 & 0103204000 & 0040230010 \\
\hline 0010230040 & 0104003200 & 0230040010 \\
\hline 0040230100 & 0013204000 & 0040230100 \\
\hline 0040100230 & 0014003200 & 0230040100 \\
\hline 0040231000 & 0003204001 & 0040231000 \\
\hline 0041000230 & 0004003201 & 0230041000 \\
\hline 0010040302 & 0304002100 & 0040300012 \\
\hline 0010300042 & 0302104000 & 0300040012 \\
\hline 0020040301 & 0304001200 & 0040300021 \\
\hline 0020300041 & 0301204000 & 0300040021 \\
\hline 0040300102 & 0304002010 & 0040300102 \\
\hline 0040100302 & 0302014000 & 0300040102 \\
\hline 0040300120 & 0210304000 & 0040300120 \\
\hline 0040120300 & 0214000300 & 0300040120 \\
\hline 0040300201 & 0304001020 & 0040300201 \\
\hline 0040200301 & 0301024000 & 0300040201 \\
\hline 0040300210 & 0120304000 & 0040300210 \\
\hline 0040210300 & 0124000300 & 0300040210 \\
\hline 0040301002 & 0304002001 & 0040301002 \\
\hline 0041000302 & 0302004001 & 0300041002 \\
\hline 0040301020 & 0200304001 & 0040301020 \\
\hline 0041020300 & 0204000301 & 0300041020 \\
\hline 0040301200 & 0020304001 & 0040301200 \\
\hline 0041200300 & 0024000301 & 0300041200 \\
\hline 0040302001 & 0304001002 & 0040302001 \\
\hline 0042000301 & 0301004002 & 0300042001 \\
\hline 0040302010 & 0100304002 & 0040302010 \\
\hline 0042010300 & 0104000302 & 0300042010 \\
\hline
\end{tabular}




\begin{tabular}{|c|c|c|}
\hline 0040302100 & 0010304002 & 0040302100 \\
\hline 0042100300 & 0014000302 & 0300042100 \\
\hline 0000040312 & 1304002000 & 0040310002 \\
\hline 0000310042 & 1302004000 & 0310040002 \\
\hline 0020040310 & 0201304000 & 0040310020 \\
\hline 0020310040 & 0204001300 & 0310040020 \\
\hline 0040310200 & 0021304000 & 0040310200 \\
\hline 0040200310 & 0024001300 & 0310040200 \\
\hline 0040312000 & 0001304002 & 0040312000 \\
\hline 0042000310 & 0004001302 & 0310042000 \\
\hline 0000040321 & 1002304000 & 0040320001 \\
\hline 0000320041 & 1004002300 & 0320040001 \\
\hline 0010040320 & 0102304000 & 0040320010 \\
\hline 0010320040 & 0104002300 & 0320040010 \\
\hline 0040320100 & 0012304000 & 0040320100 \\
\hline 0040100320 & 0014002300 & 0320040100 \\
\hline 0040321000 & 0002304001 & 0040321000 \\
\hline 0041000320 & 0004002301 & 0320041000 \\
\hline 0000041023 & 2014003000 & 0041020003 \\
\hline 0001020043 & 2013004000 & 1020040003 \\
\hline 0030041020 & 0302014000 & 0041020030 \\
\hline 0031020040 & 0304002010 & 1020040030 \\
\hline 0041020300 & 0032014000 & 0041020300 \\
\hline 0040301020 & 0034002010 & 1020040300 \\
\hline 0041023000 & 0002014003 & 0041023000 \\
\hline 0043001020 & 0004002013 & 1020043000 \\
\hline 0000041032 & 2003014000 & 0041030002 \\
\hline 0001030042 & 2004003010 & 1030040002 \\
\hline 0020041030 & 0203014000 & 0041030020 \\
\hline 0021030040 & 0204003010 & 1030040020 \\
\hline 0041030200 & 0023014000 & 0041030200 \\
\hline 0040201030 & 0024003010 & 1030040200 \\
\hline 0041032000 & 0003014002 & 0041032000 \\
\hline 0042001030 & 0004003012 & 1030042000 \\
\hline 0000041203 & 0214003000 & 0041200003 \\
\hline 0001200043 & 0213004000 & 1200040003 \\
\hline 0030041200 & 0214000300 & 0041200030 \\
\hline 0031200040 & 0210304000 & 1200040030 \\
\hline 0041200300 & 0030214000 & 0041200300 \\
\hline 0040301200 & 0034000210 & 1200040300 \\
\hline 0041203000 & 0000214003 & 0041203000 \\
\hline 0043001200 & 0004000213 & 1200043000 \\
\hline 0000041230 & 0003214000 & 0041230000 \\
\hline 0001230040 & 0004003210 & 1230040000 \\
\hline 0000041302 & 0314002000 & 0041300002 \\
\hline 0001300042 & 0312004000 & 1300040002 \\
\hline 0020041300 & 0200314000 & 0041300020 \\
\hline 0021300040 & 0204000310 & 1300040020 \\
\hline 0041300200 & 0020314000 & 0041300200 \\
\hline 0040201300 & 0024000310 & 1300040200 \\
\hline
\end{tabular}




\begin{tabular}{|c|c|c|}
\hline 0041302000 & 0000314002 & 0041302000 \\
\hline 0042001300 & 0004000312 & 1300042000 \\
\hline 0000041320 & 0002314000 & 0041320000 \\
\hline 0001320040 & 0004002310 & 1320040000 \\
\hline 0000042013 & 1024003000 & 0042010003 \\
\hline 0002010043 & 1023004000 & 2010040003 \\
\hline 0030042010 & 0301024000 & 0042010030 \\
\hline 0032010040 & 0304001020 & 2010040030 \\
\hline 0042010300 & 0031024000 & 0042010300 \\
\hline 0040302010 & 0034001020 & 2010040300 \\
\hline 0042013000 & 0001024003 & 0042013000 \\
\hline 0043002010 & 0004001023 & 2010043000 \\
\hline 0000042031 & 1003024000 & 0042030001 \\
\hline 0002030041 & 1004003020 & 2030040001 \\
\hline 0010042030 & 0103024000 & 0042030010 \\
\hline 0012030040 & 0104003020 & 2030040010 \\
\hline 0042030100 & 0013024000 & 0042030100 \\
\hline 0040102030 & 0014003020 & 2030040100 \\
\hline 0042031000 & 0003024001 & 0042031000 \\
\hline 0041002030 & 0004003021 & 2030041000 \\
\hline 0000042103 & 0124003000 & 0042100003 \\
\hline 0002100043 & 0123004000 & 2100040003 \\
\hline 0030042100 & 0124000300 & 0042100030 \\
\hline 0032100040 & 0120304000 & 2100040030 \\
\hline 0042100300 & 0030124000 & 0042100300 \\
\hline 0040302100 & 0034000120 & 2100040300 \\
\hline 0042103000 & 0000124003 & 0042103000 \\
\hline 0043002100 & 0004000123 & 2100043000 \\
\hline 0000042130 & 0003124000 & 0042130000 \\
\hline 0002130040 & 0004003120 & 2130040000 \\
\hline 0000042301 & 0324001000 & 0042300001 \\
\hline 0002300041 & 0321004000 & 2300040001 \\
\hline 0010042300 & 0100324000 & 0042300010 \\
\hline 0012300040 & 0104000320 & 2300040010 \\
\hline 0042300100 & 0010324000 & 0042300100 \\
\hline 0040102300 & 0014000320 & 2300040100 \\
\hline 0042301000 & 0000324001 & 0042301000 \\
\hline 0041002300 & 0004000321 & 2300041000 \\
\hline 0000042310 & 0001324000 & 0042310000 \\
\hline 0002310040 & 0004001320 & 2310040000 \\
\hline 0000043012 & 1034002000 & 0043010002 \\
\hline 0003010042 & 1032004000 & 3010040002 \\
\hline 0020043010 & 0201034000 & 0043010020 \\
\hline 0023010040 & 0204001030 & 3010040020 \\
\hline 0043010200 & 0021034000 & 0043010200 \\
\hline 0040203010 & 0024001030 & 3010040200 \\
\hline 0043012000 & 0001034002 & 0043012000 \\
\hline 0042003010 & 0004001032 & 3010042000 \\
\hline 0000043021 & 1002034000 & 0043020001 \\
\hline 0003020041 & 1004002030 & 3020040001 \\
\hline
\end{tabular}




\begin{tabular}{|c|c|c|}
\hline 0010043020 & 0102034000 & 0043020010 \\
\hline 0013020040 & 0104002030 & 3020040010 \\
\hline 0043020100 & 0012034000 & 0043020100 \\
\hline 0040103020 & 0014002030 & 3020040100 \\
\hline 0043021000 & 0002034001 & 0043021000 \\
\hline 0041003020 & 0004002031 & 3020041000 \\
\hline 0000043102 & 0134002000 & 0043100002 \\
\hline 0003100042 & 0132004000 & 3100040002 \\
\hline 0020043100 & 0134000200 & 0043100020 \\
\hline 0023100040 & 0130204000 & 3100040020 \\
\hline 0043100200 & 0020134000 & 0043100200 \\
\hline 0040203100 & 0024000130 & 3100040200 \\
\hline 0043102000 & 0000134002 & 0043102000 \\
\hline 0042003100 & 0004000132 & 3100042000 \\
\hline 0000043120 & 0002134000 & 0043120000 \\
\hline 0003120040 & 0004002130 & 3120040000 \\
\hline 0000043201 & 0234001000 & 0043200001 \\
\hline 0003200041 & 0231004000 & 3200040001 \\
\hline 0010043200 & 0100234000 & 0043200010 \\
\hline 0013200040 & 0104000230 & 3200040010 \\
\hline 0043200100 & 0010234000 & 0043200100 \\
\hline 0040103200 & 0014000230 & 3200040100 \\
\hline 0043201000 & 0000234001 & 0043201000 \\
\hline 0041003200 & 0004000231 & 3200041000 \\
\hline 0000043210 & 0001234000 & 0043210000 \\
\hline 0003210040 & 0004001230 & 3210040000 \\
\hline 0030100204 & 0104300200 & 0100200034 \\
\hline 0030200104 & 0100204300 & 0200100034 \\
\hline 0040100203 & 0103400200 & 0100200043 \\
\hline 0040200103 & 0100203400 & 0200100043 \\
\hline 0100200304 & 0104030200 & 0100200304 \\
\hline 0100300204 & 0100204030 & 0200100304 \\
\hline 0100200340 & 0100430200 & 0100200340 \\
\hline 0100340200 & 0100200430 & 0200100340 \\
\hline 0100200403 & 0103040200 & 0100200403 \\
\hline 0100400203 & 0100203040 & 0200100403 \\
\hline 0100203004 & 0104000203 & 0100203004 \\
\hline 0103000204 & 0100204003 & 0200103004 \\
\hline 0000100234 & 0104003200 & 0100230004 \\
\hline 0000230104 & 0103204000 & 0230100004 \\
\hline 0040100230 & 0100403200 & 0100230040 \\
\hline 0040230100 & 0103200400 & 0230100040 \\
\hline 0100230400 & 0043200100 & 0100230400 \\
\hline 0100400230 & 0040103200 & 0230100400 \\
\hline 0100234000 & 0003200104 & 0100234000 \\
\hline 0104000230 & 0000103204 & 0230104000 \\
\hline 0000100243 & 0103004200 & 0100240003 \\
\hline 0000240103 & 0104203000 & 0240100003 \\
\hline 0030100240 & 0100304200 & 0100240030 \\
\hline 0030240100 & 0104200300 & 0240100030 \\
\hline
\end{tabular}




\begin{tabular}{|c|c|c|}
\hline 0100240300 & 0034200100 & 0100240300 \\
\hline 0100300240 & 0030104200 & 0240100300 \\
\hline 0100243000 & 0004200103 & 0100243000 \\
\hline 0103000240 & 0000104203 & 0240103000 \\
\hline 0020100304 & 0104200300 & 0100300024 \\
\hline 0020300104 & 0100304200 & 0300100024 \\
\hline 0040100302 & 0102400300 & 0100300042 \\
\hline 0040300102 & 0100302400 & 0300100042 \\
\hline 0100300204 & 0104020300 & 0100300204 \\
\hline 0100200304 & 0100304020 & 0300100204 \\
\hline 0100300240 & 0100420300 & 0100300240 \\
\hline 0100240300 & 0100300420 & 0300100240 \\
\hline 0100300402 & 0102040300 & 0100300402 \\
\hline 0100400302 & 0100302040 & 0300100402 \\
\hline 0100302004 & 0104000302 & 0100302004 \\
\hline 0102000304 & 0100304002 & 0300102004 \\
\hline 0000100324 & 0104002300 & 0100320004 \\
\hline 0000320104 & 0102304000 & 0320100004 \\
\hline 0040100320 & 0100402300 & 0100320040 \\
\hline 0040320100 & 0102300400 & 0320100040 \\
\hline 0100320400 & 0042300100 & 0100320400 \\
\hline 0100400320 & 0040102300 & 0320100400 \\
\hline 0100324000 & 0002300104 & 0100324000 \\
\hline 0104000320 & 0000102304 & 0320104000 \\
\hline 0000100342 & 0102004300 & 0100340002 \\
\hline 0000340102 & 0104302000 & 0340100002 \\
\hline 0020100340 & 0100204300 & 0100340020 \\
\hline 0020340100 & 0104300200 & 0340100020 \\
\hline 0100340200 & 0024300100 & 0100340200 \\
\hline 0100200340 & 0020104300 & 0340100200 \\
\hline 0100342000 & 0004300102 & 0100342000 \\
\hline 0102000340 & 0000104302 & 0340102000 \\
\hline 0020100403 & 0103200400 & 0100400023 \\
\hline 0020400103 & 0100403200 & 0400100023 \\
\hline 0030100402 & 0102300400 & 0100400032 \\
\hline 0030400102 & 0100402300 & 0400100032 \\
\hline 0100400203 & 0103020400 & 0100400203 \\
\hline 0100200403 & 0100403020 & 0400100203 \\
\hline 0100400230 & 0100320400 & 0100400230 \\
\hline 0100230400 & 0100400320 & 0400100230 \\
\hline 0100400302 & 0102030400 & 0100400302 \\
\hline 0100300402 & 0100402030 & 0400100302 \\
\hline 0100402003 & 0103000402 & 0100402003 \\
\hline 0102000403 & 0100403002 & 0400102003 \\
\hline 0000100423 & 0103002400 & 0100420003 \\
\hline 0000420103 & 0102403000 & 0420100003 \\
\hline 0030100420 & 0100302400 & 0100420030 \\
\hline 0030420100 & 0102400300 & 0420100030 \\
\hline 0100420300 & 0032400100 & 0100420300 \\
\hline 0100300420 & 0030102400 & 0420100300 \\
\hline
\end{tabular}




\begin{tabular}{|c|c|c|}
\hline 0100423000 & 0002400103 & 0100423000 \\
\hline 0103000420 & 0000102403 & 0420103000 \\
\hline 0000100432 & 0102003400 & 0100430002 \\
\hline 0000430102 & 0103402000 & 0430100002 \\
\hline 0020100430 & 0100203400 & 0100430020 \\
\hline 0020430100 & 0103400200 & 0430100020 \\
\hline 0100430200 & 0023400100 & 0100430200 \\
\hline 0100200430 & 0020103400 & 0430100200 \\
\hline 0100432000 & 0003400102 & 0100432000 \\
\hline 0102000430 & 0000103402 & 0430102000 \\
\hline 0000102034 & 0104003020 & 0102030004 \\
\hline 0002030104 & 0103024000 & 2030100004 \\
\hline 0040102030 & 0100403020 & 0102030040 \\
\hline 0042030100 & 0103020400 & 2030100040 \\
\hline 0102030400 & 0043020100 & 0102030400 \\
\hline 0100402030 & 0040103020 & 2030100400 \\
\hline 0102034000 & 0003020104 & 0102034000 \\
\hline 0104002030 & 0000103024 & 2030104000 \\
\hline 0000102043 & 0103004020 & 0102040003 \\
\hline 0002040103 & 0104023000 & 2040100003 \\
\hline 0030102040 & 0100304020 & 0102040030 \\
\hline 0032040100 & 0104020300 & 2040100030 \\
\hline 0102040300 & 0034020100 & 0102040300 \\
\hline 0100302040 & 0030104020 & 2040100300 \\
\hline 0102043000 & 0004020103 & 0102043000 \\
\hline 0103002040 & 0000104023 & 2040103000 \\
\hline 0000102340 & 0004320100 & 0102340000 \\
\hline 0002340100 & 0000104320 & 2340100000 \\
\hline 0000102430 & 0003420100 & 0102430000 \\
\hline 0002430100 & 0000103420 & 2430100000 \\
\hline 0000103042 & 0102004030 & 0103040002 \\
\hline 0003040102 & 0104032000 & 3040100002 \\
\hline 0020103040 & 0100204030 & 0103040020 \\
\hline 0023040100 & 0104030200 & 3040100020 \\
\hline 0103040200 & 0024030100 & 0103040200 \\
\hline 0100203040 & 0020104030 & 3040100200 \\
\hline 0103042000 & 0004030102 & 0103042000 \\
\hline 0102003040 & 0000104032 & 3040102000 \\
\hline 0000103240 & 0004230100 & 0103240000 \\
\hline 0003240100 & 0000104230 & 3240100000 \\
\hline 0000120304 & 0302104000 & 0120300004 \\
\hline 0000300124 & 0304002100 & 0300120004 \\
\hline 0040120300 & 0302100400 & 0120300040 \\
\hline 0040300120 & 0300402100 & 0300120040 \\
\hline 0120300400 & 0040302100 & 0120300400 \\
\hline 0120400300 & 0042100300 & 0300120400 \\
\hline 0120304000 & 0000302104 & 0120304000 \\
\hline 0124000300 & 0002100304 & 0300124000 \\
\hline 0000120340 & 0004302100 & 0120340000 \\
\hline 0000340120 & 0002104300 & 0340120000 \\
\hline
\end{tabular}




\begin{tabular}{|c|c|c|}
\hline 0000120403 & 0402103000 & 0120400003 \\
\hline 0000400123 & 0403002100 & 0400120003 \\
\hline 0030120400 & 0300402100 & 0120400030 \\
\hline 0030400120 & 0302100400 & 0400120030 \\
\hline 0120400300 & 0030402100 & 0120400300 \\
\hline 0120300400 & 0032100400 & 0400120300 \\
\hline 0120403000 & 0000402103 & 0120403000 \\
\hline 0123000400 & 0002100403 & 0400123000 \\
\hline 0000120430 & 0003402100 & 0120430000 \\
\hline 0000430120 & 0002103400 & 0430120000 \\
\hline 0000123040 & 0004032100 & 0123040000 \\
\hline 0003040120 & 0002104030 & 3040120000 \\
\hline 0000123400 & 0000432100 & 0123400000 \\
\hline 0003400120 & 0002100430 & 3400120000 \\
\hline 0000124030 & 0003042100 & 0124030000 \\
\hline 0004030120 & 0002103040 & 4030120000 \\
\hline 0000124300 & 0000342100 & 0124300000 \\
\hline 0004300120 & 0002100340 & 4300120000 \\
\hline 0000130204 & 0203104000 & 0130200004 \\
\hline 0000200134 & 0204003100 & 0200130004 \\
\hline 0040130200 & 0203100400 & 0130200040 \\
\hline 0040200130 & 0200403100 & 0200130040 \\
\hline 0130200400 & 0040203100 & 0130200400 \\
\hline 0130400200 & 0043100200 & 0200130400 \\
\hline 0130204000 & 0000203104 & 0130204000 \\
\hline 0134000200 & 0003100204 & 0200134000 \\
\hline 0000130240 & 0004203100 & 0130240000 \\
\hline 0000240130 & 0003104200 & 0240130000 \\
\hline 0000130402 & 0403102000 & 0130400002 \\
\hline 0000400132 & 0402003100 & 0400130002 \\
\hline 0020130400 & 0200403100 & 0130400020 \\
\hline 0020400130 & 0203100400 & 0400130020 \\
\hline 0130400200 & 0020403100 & 0130400200 \\
\hline 0130200400 & 0023100400 & 0400130200 \\
\hline 0130402000 & 0000403102 & 0130402000 \\
\hline 0132000400 & 0003100402 & 0400132000 \\
\hline 0000130420 & 0002403100 & 0130420000 \\
\hline 0000420130 & 0003102400 & 0420130000 \\
\hline 0000132040 & 0004023100 & 0132040000 \\
\hline 0002040130 & 0003104020 & 2040130000 \\
\hline 0000132400 & 0000423100 & 0132400000 \\
\hline 0002400130 & 0003100420 & 2400130000 \\
\hline 0000134020 & 0002043100 & 0134020000 \\
\hline 0004020130 & 0003102040 & 4020130000 \\
\hline 0000134200 & 0000243100 & 0134200000 \\
\hline 0004200130 & 0003100240 & 4200130000 \\
\hline 0000140203 & 0204103000 & 0140200003 \\
\hline 0000200143 & 0203004100 & 0200140003 \\
\hline 0030140200 & 0204100300 & 0140200030 \\
\hline 0030200140 & 0200304100 & 0200140030 \\
\hline
\end{tabular}




\begin{tabular}{|c|c|c|}
\hline 0140200300 & 0030204100 & 0140200300 \\
\hline 0140300200 & 0034100200 & 0200140300 \\
\hline 0140203000 & 0000204103 & 0140203000 \\
\hline 0143000200 & 0004100203 & 0200143000 \\
\hline 0000140230 & 0003204100 & 0140230000 \\
\hline 0000230140 & 0004103200 & 0230140000 \\
\hline 0000140302 & 0304102000 & 0140300002 \\
\hline 0000300142 & 0302004100 & 0300140002 \\
\hline 0020140300 & 0200304100 & 0140300020 \\
\hline 0020300140 & 0204100300 & 0300140020 \\
\hline 0140300200 & 0020304100 & 0140300200 \\
\hline 0140200300 & 0024100300 & 0300140200 \\
\hline 0140302000 & 0000304102 & 0140302000 \\
\hline 0142000300 & 0004100302 & 0300142000 \\
\hline 0000140320 & 0002304100 & 0140320000 \\
\hline 0000320140 & 0004102300 & 0320140000 \\
\hline 0000142030 & 0003024100 & 0142030000 \\
\hline 0002030140 & 0004103020 & 2030140000 \\
\hline 0000142300 & 0000324100 & 0142300000 \\
\hline 0002300140 & 0004100320 & 2300140000 \\
\hline 0000143020 & 0002034100 & 0143020000 \\
\hline 0003020140 & 0004102030 & 3020140000 \\
\hline 0000143200 & 0000234100 & 0143200000 \\
\hline 0003200140 & 0004100230 & 3200140000 \\
\hline 0010200304 & 0204100300 & 0200300014 \\
\hline 0010300204 & 0200304100 & 0300200014 \\
\hline 0040200301 & 0201400300 & 0200300041 \\
\hline 0040300201 & 0200301400 & 0300200041 \\
\hline 0100200304 & 0204010300 & 0200300104 \\
\hline 0100300204 & 0200304010 & 0300200104 \\
\hline 0140200300 & 0200410300 & 0200300140 \\
\hline 0140300200 & 0200300410 & 0300200140 \\
\hline 0200300401 & 0201040300 & 0200300401 \\
\hline 0200400301 & 0200301040 & 0300200401 \\
\hline 0200301004 & 0204000301 & 0200301004 \\
\hline 0201000304 & 0200304001 & 0300201004 \\
\hline 0000200314 & 0204001300 & 0200310004 \\
\hline 0000310204 & 0201304000 & 0310200004 \\
\hline 0040200310 & 0200401300 & 0200310040 \\
\hline 0040310200 & 0201300400 & 0310200040 \\
\hline 0200310400 & 0041300200 & 0200310400 \\
\hline 0200400310 & 0040201300 & 0310200400 \\
\hline 0200314000 & 0001300204 & 0200314000 \\
\hline 0204000310 & 0000201304 & 0310204000 \\
\hline 0000200341 & 0201004300 & 0200340001 \\
\hline 0000340201 & 0204301000 & 0340200001 \\
\hline 0010200340 & 0104300200 & 0200340010 \\
\hline 0010340200 & 0100204300 & 0340200010 \\
\hline 0100200340 & 0014300200 & 0200340100 \\
\hline 0100340200 & 0010204300 & 0340200100 \\
\hline
\end{tabular}




\begin{tabular}{|c|c|c|}
\hline 0200341000 & 0004300201 & 0200341000 \\
\hline 0201000340 & 0000204301 & 0340201000 \\
\hline 0010200403 & 0203100400 & 0200400013 \\
\hline 0010400203 & 0200403100 & 0400200013 \\
\hline 0030200401 & 0201300400 & 0200400031 \\
\hline 0030400201 & 0200401300 & 0400200031 \\
\hline 0100200403 & 0203010400 & 0200400103 \\
\hline 0100400203 & 0200403010 & 0400200103 \\
\hline 0130200400 & 0200310400 & 0200400130 \\
\hline 0130400200 & 0200400310 & 0400200130 \\
\hline 0200400301 & 0201030400 & 0200400301 \\
\hline 0200300401 & 0200401030 & 0400200301 \\
\hline 0200401003 & 0203000401 & 0200401003 \\
\hline 0201000403 & 0200403001 & 0400201003 \\
\hline 0000200413 & 0203001400 & 0200410003 \\
\hline 0000410203 & 0201403000 & 0410200003 \\
\hline 0030200410 & 0200301400 & 0200410030 \\
\hline 0030410200 & 0201400300 & 0410200030 \\
\hline 0200410300 & 0031400200 & 0200410300 \\
\hline 0200300410 & 0030201400 & 0410200300 \\
\hline 0200413000 & 0001400203 & 0200413000 \\
\hline 0203000410 & 0000201403 & 0410203000 \\
\hline 0000200431 & 0201003400 & 0200430001 \\
\hline 0000430201 & 0203401000 & 0430200001 \\
\hline 0010200430 & 0103400200 & 0200430010 \\
\hline 0010430200 & 0100203400 & 0430200010 \\
\hline 0100200430 & 0013400200 & 0200430100 \\
\hline 0100430200 & 0010203400 & 0430200100 \\
\hline 0200431000 & 0003400201 & 0200431000 \\
\hline 0201000430 & 0000203401 & 0430201000 \\
\hline 0000201034 & 0204003010 & 0201030004 \\
\hline 0001030204 & 0203014000 & 1030200004 \\
\hline 0040201030 & 0200403010 & 0201030040 \\
\hline 0041030200 & 0203010400 & 1030200040 \\
\hline 0201030400 & 0043010200 & 0201030400 \\
\hline 0200401030 & 0040203010 & 1030200400 \\
\hline 0201034000 & 0003010204 & 0201034000 \\
\hline 0204001030 & 0000203014 & 1030204000 \\
\hline 0000201043 & 0203004010 & 0201040003 \\
\hline 0001040203 & 0204013000 & 1040200003 \\
\hline 0030201040 & 0200304010 & 0201040030 \\
\hline 0031040200 & 0204010300 & 1040200030 \\
\hline 0201040300 & 0034010200 & 0201040300 \\
\hline 0200301040 & 0030204010 & 1040200300 \\
\hline 0201043000 & 0004010203 & 0201043000 \\
\hline 0203001040 & 0000204013 & 1040203000 \\
\hline 0000201340 & 0004310200 & 0201340000 \\
\hline 0001340200 & 0000204310 & 1340200000 \\
\hline 0000201430 & 0003410200 & 0201430000 \\
\hline 0001430200 & 0000203410 & 1430200000 \\
\hline
\end{tabular}




\begin{tabular}{|c|c|c|}
\hline 0000203041 & 0201004030 & 0203040001 \\
\hline 0003040201 & 0204031000 & 3040200001 \\
\hline 0010203040 & 0104030200 & 0203040010 \\
\hline 0013040200 & 0100204030 & 3040200010 \\
\hline 0100203040 & 0014030200 & 0203040100 \\
\hline 0103040200 & 0010204030 & 3040200100 \\
\hline 0203041000 & 0004030201 & 0203041000 \\
\hline 0201003040 & 0000204031 & 3040201000 \\
\hline 0000203140 & 0004130200 & 0203140000 \\
\hline 0003140200 & 0000204130 & 3140200000 \\
\hline 0000210304 & 0301204000 & 0210300004 \\
\hline 0000300214 & 0304001200 & 0300210004 \\
\hline 0040210300 & 0301200400 & 0210300040 \\
\hline 0040300210 & 0300401200 & 0300210040 \\
\hline 0210300400 & 0040301200 & 0210300400 \\
\hline 0210400300 & 0041200300 & 0300210400 \\
\hline 0210304000 & 0000301204 & 0210304000 \\
\hline 0214000300 & 0001200304 & 0300214000 \\
\hline 0000210340 & 0004301200 & 0210340000 \\
\hline 0000340210 & 0001204300 & 0340210000 \\
\hline 0000210403 & 0401203000 & 0210400003 \\
\hline 0000400213 & 0403001200 & 0400210003 \\
\hline 0030210400 & 0300401200 & 0210400030 \\
\hline 0030400210 & 0301200400 & 0400210030 \\
\hline 0210400300 & 0030401200 & 0210400300 \\
\hline 0210300400 & 0031200400 & 0400210300 \\
\hline 0210403000 & 0000401203 & 0210403000 \\
\hline 0213000400 & 0001200403 & 0400213000 \\
\hline 0000210430 & 0003401200 & 0210430000 \\
\hline 0000430210 & 0001203400 & 0430210000 \\
\hline 0000213040 & 0004031200 & 0213040000 \\
\hline 0003040210 & 0001204030 & 3040210000 \\
\hline 0000213400 & 0000431200 & 0213400000 \\
\hline 0003400210 & 0001200430 & 3400210000 \\
\hline 0000214030 & 0003041200 & 0214030000 \\
\hline 0004030210 & 0001203040 & 4030210000 \\
\hline 0000214300 & 0000341200 & 0214300000 \\
\hline 0004300210 & 0001200340 & 4300210000 \\
\hline 0000230401 & 0403201000 & 0230400001 \\
\hline 0000400231 & 0401003200 & 0400230001 \\
\hline 0010230400 & 0100403200 & 0230400010 \\
\hline 0010400230 & 0103200400 & 0400230010 \\
\hline 0100230400 & 0010403200 & 0230400100 \\
\hline 0100400230 & 0013200400 & 0400230100 \\
\hline 0230401000 & 0000403201 & 0230401000 \\
\hline 0231000400 & 0003200401 & 0400231000 \\
\hline 0000230410 & 0001403200 & 0230410000 \\
\hline 0000410230 & 0003201400 & 0410230000 \\
\hline 0000231040 & 0004013200 & 0231040000 \\
\hline 0001040230 & 0003204010 & 1040230000 \\
\hline
\end{tabular}




\begin{tabular}{|c|c|c|}
\hline 0000231400 & 0000413200 & 0231400000 \\
\hline 0001400230 & 0003200410 & 1400230000 \\
\hline 0000234010 & 0001043200 & 0234010000 \\
\hline 0004010230 & 0003201040 & 4010230000 \\
\hline 0000240301 & 0304201000 & 0240300001 \\
\hline 0000300241 & 0301004200 & 0300240001 \\
\hline 0010240300 & 0100304200 & 0240300010 \\
\hline 0010300240 & 0104200300 & 0300240010 \\
\hline 0100240300 & 0010304200 & 0240300100 \\
\hline 0100300240 & 0014200300 & 0300240100 \\
\hline 0240301000 & 0000304201 & 0240301000 \\
\hline 0241000300 & 0004200301 & 0300241000 \\
\hline 0000240310 & 0001304200 & 0240310000 \\
\hline 0000310240 & 0004201300 & 0310240000 \\
\hline 0000241030 & 0003014200 & 0241030000 \\
\hline 0001030240 & 0004203010 & 1030240000 \\
\hline 0000241300 & 0000314200 & 0241300000 \\
\hline 0001300240 & 0004200310 & 1300240000 \\
\hline 0000243010 & 0001034200 & 0243010000 \\
\hline 0003010240 & 0004201030 & 3010240000 \\
\hline 0010300402 & 0302100400 & 0300400012 \\
\hline 0010400302 & 0300402100 & 0400300012 \\
\hline 0020300401 & 0301200400 & 0300400021 \\
\hline 0020400301 & 0300401200 & 0400300021 \\
\hline 0100300402 & 0302010400 & 0300400102 \\
\hline 0100400302 & 0300402010 & 0400300102 \\
\hline 0120300400 & 0210400300 & 0300400120 \\
\hline 0120400300 & 0210300400 & 0400300120 \\
\hline 0200300401 & 0301020400 & 0300400201 \\
\hline 0200400301 & 0300401020 & 0400300201 \\
\hline 0300401002 & 0302000401 & 0300401002 \\
\hline 0301000402 & 0300402001 & 0400301002 \\
\hline 0000300412 & 0302001400 & 0300410002 \\
\hline 0000410302 & 0301402000 & 0410300002 \\
\hline 0020300410 & 0201400300 & 0300410020 \\
\hline 0020410300 & 0200301400 & 0410300020 \\
\hline 0200300410 & 0021400300 & 0300410200 \\
\hline 0200410300 & 0020301400 & 0410300200 \\
\hline 0300412000 & 0001400302 & 0300412000 \\
\hline 0302000410 & 0000301402 & 0410302000 \\
\hline 0000300421 & 0301002400 & 0300420001 \\
\hline 0000420301 & 0302401000 & 0420300001 \\
\hline 0010300420 & 0102400300 & 0300420010 \\
\hline 0010420300 & 0100302400 & 0420300010 \\
\hline 0100300420 & 0012400300 & 0300420100 \\
\hline 0100420300 & 0010302400 & 0420300100 \\
\hline 0300421000 & 0002400301 & 0300421000 \\
\hline 0301000420 & 0000302401 & 0420301000 \\
\hline 0000301024 & 0304002010 & 0301020004 \\
\hline 0001020304 & 0302014000 & 1020300004 \\
\hline
\end{tabular}




\begin{tabular}{|c|c|c|}
\hline 0040301020 & 0300402010 & 0301020040 \\
\hline 0041020300 & 0302010400 & 1020300040 \\
\hline 0301020400 & 0042010300 & 0301020400 \\
\hline 0300401020 & 0040302010 & 1020300400 \\
\hline 0301024000 & 0002010304 & 0301024000 \\
\hline 0304001020 & 0000302014 & 1020304000 \\
\hline 0000301042 & 0302004010 & 0301040002 \\
\hline 0001040302 & 0304012000 & 1040300002 \\
\hline 0020301040 & 0204010300 & 0301040020 \\
\hline 0021040300 & 0200304010 & 1040300020 \\
\hline 0200301040 & 0024010300 & 0301040200 \\
\hline 0201040300 & 0020304010 & 1040300200 \\
\hline 0301042000 & 0004010302 & 0301042000 \\
\hline 0302001040 & 0000304012 & 1040302000 \\
\hline 0000301240 & 0004210300 & 0301240000 \\
\hline 0001240300 & 0000304210 & 1240300000 \\
\hline 0000301420 & 0002410300 & 0301420000 \\
\hline 0001420300 & 0000302410 & 1420300000 \\
\hline 0000302041 & 0301004020 & 0302040001 \\
\hline 0002040301 & 0304021000 & 2040300001 \\
\hline 0010302040 & 0104020300 & 0302040010 \\
\hline 0012040300 & 0100304020 & 2040300010 \\
\hline 0100302040 & 0014020300 & 0302040100 \\
\hline 0102040300 & 0010304020 & 2040300100 \\
\hline 0302041000 & 0004020301 & 0302041000 \\
\hline 0301002040 & 0000304021 & 2040301000 \\
\hline 0000302140 & 0004120300 & 0302140000 \\
\hline 0002140300 & 0000304120 & 2140300000 \\
\hline 0000310402 & 0401302000 & 0310400002 \\
\hline 0000400312 & 0402001300 & 0400310002 \\
\hline 0020310400 & 0200401300 & 0310400020 \\
\hline 0020400310 & 0201300400 & 0400310020 \\
\hline 0200310400 & 0020401300 & 0310400200 \\
\hline 0200400310 & 0021300400 & 0400310200 \\
\hline 0310402000 & 0000401302 & 0310402000 \\
\hline 0312000400 & 0001300402 & 0400312000 \\
\hline 0000310420 & 0002401300 & 0310420000 \\
\hline 0000420310 & 0001302400 & 0420310000 \\
\hline 0000312040 & 0004021300 & 0312040000 \\
\hline 0002040310 & 0001304020 & 2040310000 \\
\hline 0000312400 & 0000421300 & 0312400000 \\
\hline 0002400310 & 0001300420 & 2400310000 \\
\hline 0000314020 & 0002041300 & 0314020000 \\
\hline 0004020310 & 0001302040 & 4020310000 \\
\hline 0000320401 & 0402301000 & 0320400001 \\
\hline 0000400321 & 0401002300 & 0400320001 \\
\hline 0010320400 & 0100402300 & 0320400010 \\
\hline 0010400320 & 0102300400 & 0400320010 \\
\hline 0100320400 & 0010402300 & 0320400100 \\
\hline 0100400320 & 0012300400 & 0400320100 \\
\hline
\end{tabular}




\begin{tabular}{|c|c|c|}
\hline 0320401000 & 0000402301 & 0320401000 \\
\hline 0321000400 & 0002300401 & 0400321000 \\
\hline 0000320410 & 0001402300 & 0320410000 \\
\hline 0000410320 & 0002301400 & 0410320000 \\
\hline 0000321040 & 0004012300 & 0321040000 \\
\hline 0001040320 & 0002304010 & 1040320000 \\
\hline 0000321400 & 0000412300 & 0321400000 \\
\hline 0001400320 & 0002300410 & 1400320000 \\
\hline 0000324010 & 0001042300 & 0324010000 \\
\hline 0004010320 & 0002301040 & 4010320000 \\
\hline 0000341020 & 0002014300 & 0341020000 \\
\hline 0001020340 & 0004302010 & 1020340000 \\
\hline 0000342010 & 0001024300 & 0342010000 \\
\hline 0002010340 & 0004301020 & 2010340000 \\
\hline 0000401023 & 0403002010 & 0401020003 \\
\hline 0001020403 & 0402013000 & 1020400003 \\
\hline 0030401020 & 0302010400 & 0401020030 \\
\hline 0031020400 & 0300402010 & 1020400030 \\
\hline 0300401020 & 0032010400 & 0401020300 \\
\hline 0301020400 & 0030402010 & 1020400300 \\
\hline 0401023000 & 0002010403 & 0401023000 \\
\hline 0403001020 & 0000402013 & 1020403000 \\
\hline 0000401032 & 0402003010 & 0401030002 \\
\hline 0001030402 & 0403012000 & 1030400002 \\
\hline 0020401030 & 0203010400 & 0401030020 \\
\hline 0021030400 & 0200403010 & 1030400020 \\
\hline 0200401030 & 0023010400 & 0401030200 \\
\hline 0201030400 & 0020403010 & 1030400200 \\
\hline 0401032000 & 0003010402 & 0401032000 \\
\hline 0402001030 & 0000403012 & 1030402000 \\
\hline 0000401230 & 0003210400 & 0401230000 \\
\hline 0001230400 & 0000403210 & 1230400000 \\
\hline 0000401320 & 0002310400 & 0401320000 \\
\hline 0001320400 & 0000402310 & 1320400000 \\
\hline 0000402031 & 0401003020 & 0402030001 \\
\hline 0002030401 & 0403021000 & 2030400001 \\
\hline 0010402030 & 0103020400 & 0402030010 \\
\hline 0012030400 & 0100403020 & 2030400010 \\
\hline 0100402030 & 0013020400 & 0402030100 \\
\hline 0102030400 & 0010403020 & 2030400100 \\
\hline 0402031000 & 0003020401 & 0402031000 \\
\hline 0401002030 & 0000403021 & 2030401000 \\
\hline 0000402130 & 0003120400 & 0402130000 \\
\hline 0002130400 & 0000403120 & 2130400000 \\
\hline 0000412030 & 0003021400 & 0412030000 \\
\hline 0002030410 & 0001403020 & 2030410000 \\
\hline 0000413020 & 0002031400 & 0413020000 \\
\hline 0003020410 & 0001402030 & 3020410000 \\
\hline 0000421030 & 0003012400 & 0421030000 \\
\hline 0001030420 & 0002403010 & 1030420000 \\
\hline
\end{tabular}




$\begin{array}{lll}0000423010 & 0001032400 & 0423010000 \\ 0003010420 & 0002401030 & 3010420000 \\ 0000431020 & 0002013400 & 0431020000 \\ 0001020430 & 0003402010 & 1020430000 \\ 0000432010 & 0001023400 & 0432010000 \\ 0002010430 & 0003401020 & 2010430000 \\ 0001023040 & 0004032010 & 1023040000 \\ 0003041020 & 0002014030 & 3041020000 \\ 0001024030 & 0003042010 & 1024030000 \\ 0004031020 & 0002013040 & 4031020000 \\ 0001032040 & 0004023010 & 1032040000 \\ 0002041030 & 0003014020 & 2041030000 \\ 0001034020 & 0002043010 & 1034020000 \\ 0004021030 & 0003012040 & 4021030000 \\ 0001042030 & 0003024010 & 1042030000 \\ 0002031040 & 0004013020 & 2031040000 \\ 0001043020 & 0002034010 & 1043020000 \\ 0003021040 & 0004012030 & 3021040000\end{array}$

Local network calculations around isomer 6:B: Single point DFT calculations at B3LYP-D3BJ/Def2-TZVPPD/CPCM (chloroform)

Vertex list:

\begin{tabular}{|c|c|}
\hline isomer & energy \\
\hline 0000104032 & 63.5355 \\
\hline 0000204031 & 51.4821 \\
\hline 0004030102 & 63.5355 \\
\hline 0004030201 & 51.4821 \\
\hline 0010024030 & 0.2258 \\
\hline 0010200403 & 33.1624 \\
\hline 0010304002 & 66.1918 \\
\hline 0010400203 & 33.1624 \\
\hline 0014000302 & 66.1918 \\
\hline 0014030020 & 0.2258 \\
\hline 0020100403 & 44.4106 \\
\hline 0020304001 & 58.6087 \\
\hline 0020400103 & 44.4106 \\
\hline 0024000301 & 58.608 \\
\hline 0102003040 & 39.251 \\
\hline 0103042000 & 39.251 \\
\hline 0201003040 & 36.693 \\
\hline 0203041000 & 36.693 \\
\hline 0301002004 & 50.010 \\
\hline 0302001004 & 50.010 \\
\hline
\end{tabular}

Edge list: 


\begin{tabular}{|c|c|c|c|}
\hline isomer1 & ier2 & TS energy & \\
\hline 0010024030 & 0103042000 & 0024030010 & 61.1170 \\
\hline 0010024030 & 0201003040 & 1003040200 & 66.8257 \\
\hline 0010024030 & 0302001004 & 0010024030 & 61.8272 \\
\hline 0014030020 & 0102003040 & 2003040100 & 61.1170 \\
\hline 0014030020 & 0203041000 & 0014030020 & 66.8257 \\
\hline 0014030020 & 0301002004 & 0020014030 & 61.8272 \\
\hline 0102003040 & 0000104032 & 0104030002 & 114.2272 \\
\hline 0102003040 & 0014030020 & 2003040100 & 61.1170 \\
\hline 0102003040 & 0020100403 & 0020100403 & 83.8233 \\
\hline 0103042000 & 0004030102 & 0103042000 & 114.2272 \\
\hline 0103042000 & 0010024030 & 0024030010 & 61.1170 \\
\hline 0103042000 & 0020400103 & 0100020403 & 83.8233 \\
\hline 0201003040 & 0000204031 & 0204030001 & 115.2348 \\
\hline 0201003040 & 0010024030 & 1003040200 & 66.8257 \\
\hline 0201003040 & 0010200403 & 0010200403 & 86.7189 \\
\hline 0203041000 & 0004030201 & 0203041000 & 115.2348 \\
\hline 0203041000 & 0010400203 & 0200010403 & 86.7189 \\
\hline 0203041000 & 0014030020 & 0014030020 & 66.8257 \\
\hline 0301002004 & 0010304002 & 0010304002 & 122.0959 \\
\hline 0301002004 & 0014030020 & 0020014030 & 61.8272 \\
\hline 0301002004 & 0024000301 & 0300024001 & 108.9763 \\
\hline 0302001004 & 0010024030 & 0010024030 & 61.8272 \\
\hline 0302001004 & 0014000302 & 0300014002 & 122.0959 \\
\hline 0302001004 & 0020304001 & 0020304001 & 108.9763 \\
\hline
\end{tabular}

Local network calculations around isomer 6:C: Single point DFT calculations at B3LYP-D3BJ/Def2-TZVPPD/CPCM (chloroform)

Vertex list:

\begin{tabular}{lrl} 
isomer & \multicolumn{2}{c}{ energy } \\
\hline------- & \\
0000204013 & 36.9500 \\
0000304012 & & 61.1588 \\
0004010203 & & 36.9500 \\
0004010302 & & 61.1588 \\
0020034010 & & 0.8602 \\
0020104003 & & 47.1967 \\
0020300401 & & 50.7367 \\
0020400301 & & 50.7367 \\
0024000103 & 47.1967 \\
0024010030 & & 0.8602 \\
0030104002 & 62.3393 \\
0030200401 & 55.7762 \\
0030400201 & 55.7762 \\
0034000102 & 62.3393 \\
0102003004 & 49.4000 \\
0103002004 & 49.4000 \\
0201043000 & 38.4392
\end{tabular}




$\begin{array}{lll}0203001040 & 38.4392 \\ 0301042000 & 41.7157 \\ 0302001040 & 41.7157\end{array}$

Edge list:

\begin{tabular}{lccll} 
isomer1 & isomer2 & trans.state TS energy & \\
\hline----- & ---- & & \\
0020034010 & 0103002004 & 0020034010 & 70.1812 \\
0020034010 & 0201043000 & 0034010020 & 66.4571 \\
0020034010 & 0302001040 & 2001040300 & 57.6082 \\
0024010030 & 0102003004 & 0030024010 & 70.1812 \\
0024010030 & 0203001040 & 3001040200 & 66.4571 \\
0024010030 & 0301042000 & 0024010030 & 57.6082 \\
0102003004 & 0020104003 & 0020104003 & 90.9859 \\
0102003004 & 0024010030 & 0030024010 & 70.1812 \\
0102003004 & 0034000102 & 0100034002 & 113.3695 \\
0103002004 & 0020034010 & 0020034010 & 70.1812 \\
0103002004 & 0024000103 & 0100024003 & 90.9859 \\
0103002004 & 0030104002 & 0030104002 & 113.3695 \\
0201043000 & 0004010203 & 0201043000 & 93.4169 \\
0201043000 & 0020034010 & 0034010020 & 66.4571 \\
0201043000 & 0030400201 & 0200030401 & 108.7994 \\
0203001040 & 0000204013 & 0204010003 & 93.4169 \\
0203001040 & 0024010030 & 3001040200 & 66.4571 \\
0203001040 & 0030200401 & 0030200401 & 108.7994 \\
0301042000 & 0004010302 & 0301042000 & 123.2794 \\
0301042000 & 0020400301 & 0300020401 & 96.0795 \\
0301042000 & 0024010030 & 0024010030 & 57.6082 \\
0302001040 & 0000304012 & 0304010002 & 123.2794 \\
0302001040 & 0020034010 & 2001040300 & 57.6082 \\
0302001040 & 0020300401 & 0020300401 & 96.0795
\end{tabular}

Local network calculations around isomer 6:D: Single point DFT calculations at B3LYP-D3BJ/Def2-TZVPPD/CPCM (chloroform)

Vertex list:

\begin{tabular}{ll} 
isomer & \multicolumn{2}{c}{ energy } \\
----- & \\
0000103024 & 48.2782 \\
0000403021 & 60.6323 \\
0003020104 & 48.2782 \\
0003020401 & 60.6323 \\
0010043020 & 1.0010 \\
0010203004 & 35.0265 \\
0010300402 & 56.5535 \\
0010400302 & 56.5535 \\
0013000204 & 35.0265
\end{tabular}




$\begin{array}{lll}0013020040 & 1.0010 \\ 0040100302 & 54.8403 \\ 0040203001 & 53.9825 \\ 0040300102 & 54.8403 \\ 0043000201 & 53.9825 \\ 0102034000 & 45.8243 \\ 0104002030 & 45.8243 \\ 0201004003 & 45.3202 \\ 0204001003 & 45.3202 \\ 0401002030 & 55.2492 \\ 0402031000 & 55.2492\end{array}$

Edge list:

\begin{tabular}{lrllll} 
isomer1 & isomer2 & \multicolumn{3}{c}{ trans.state TS energy } & \\
\hdashline 0010043020 & 0102034000 & 0043020010 & 49.6540 \\
0010043020 & 0204001003 & 0010043020 & 76.7119 \\
0010043020 & 0401002030 & 1002030400 & 60.1163 \\
0013020040 & 0104002030 & 3020040010 & 49.6540 \\
0013020040 & 0201004003 & 0040013020 & 76.7119 \\
0013020040 & 0402031000 & 0013020040 & 60.1163 \\
0102034000 & 0003020104 & 0102034000 & 91.3828 \\
0102034000 & 0010043020 & 0043020010 & 49.6540 \\
0102034000 & 0040300102 & 0100040302 & 116.4419 \\
0104002030 & 0000103024 & 0103020004 & 91.3828 \\
0104002030 & 0013020040 & 3020040010 & 49.6540 \\
0104002030 & 0040100302 & 0040100302 & 116.4419 \\
0201004003 & 0010203004 & 0010203004 & 99.3436 \\
0201004003 & 0013020040 & 0040013020 & 76.7119 \\
0201004003 & 0043000201 & 0200043001 & 118.0118 \\
0204001003 & 0010043020 & 0010043020 & 76.7119 \\
0204001003 & 0013000204 & 0200013004 & 99.3436 \\
0204001003 & 0040203001 & 0040203001 & 118.0118 \\
0401002030 & 0000403021 & 0403020001 & 106.4444 \\
0401002030 & 0010043020 & 1002030400 & 60.1163 \\
0401002030 & 0010400302 & 0010400302 & 115.3164 \\
0402031000 & 0003020401 & 0402031000 & 106.4444 \\
0402031000 & 0010300402 & 0400010302 & 115.3164 \\
0402031000 & 0013020040 & 0013020040 & 60.1163
\end{tabular}

Local network calculations around isomer 6:E: Single point DFT calculations at B3LYP-D3BJ/Def2-TZVPPD/CPCM (chloroform)

Vertex list:

isomer

energy

0000203014

30.4801 


$\begin{array}{lll}0000403012 & 59.0220 \\ 0003010204 & 30.4801 \\ 0003010402 & 59.0220 \\ 0020043010 & 1.6379 \\ 0020103004 & 46.2749 \\ 0020300401 & 50.7367 \\ 0020400301 & 50.7367 \\ 0023000104 & 46.2749 \\ 0023010040 & 1.6379 \\ 0040103002 & 58.8846 \\ 0040200301 & 49.8008 \\ 0040300201 & 49.8008 \\ 0043000102 & 58.8846 \\ 0102004003 & 48.0778 \\ 0104002003 & 48.0778 \\ 0201034000 & 33.4910 \\ 0204001030 & 33.4910 \\ 0401032000 & 55.7767 \\ 0402001030 & 55.7767\end{array}$

Edge list:

\begin{tabular}{|c|c|c|c|}
\hline isomer1 & er2 & TS energy & \\
\hline 0020043010 & 0104002003 & 0020043010 & 59.1157 \\
\hline 0020043010 & 0201034000 & 0043010020 & 68.4233 \\
\hline 0020043010 & 0402001030 & 2001030400 & 56.9143 \\
\hline 0023010040 & 0102004003 & 0040023010 & 59.1157 \\
\hline 0023010040 & 0204001030 & 3010040020 & 68.4233 \\
\hline 0023010040 & 0401032000 & 0023010040 & 56.9143 \\
\hline 0102004003 & 0020103004 & 0020103004 & 90.1591 \\
\hline 0102004003 & 0023010040 & 0040023010 & 59.1157 \\
\hline 0102004003 & 0043000102 & 0100043002 & 113.5370 \\
\hline 0104002003 & 0020043010 & 0020043010 & 59.1157 \\
\hline 0104002003 & 0023000104 & 0100023004 & 90.1591 \\
\hline 0104002003 & 0040103002 & 0040103002 & 113.5370 \\
\hline 0201034000 & 0003010204 & 0201034000 & 88.6225 \\
\hline 0201034000 & 0020043010 & 0043010020 & 68.4233 \\
\hline 0201034000 & 0040300201 & 0200040301 & 107.8277 \\
\hline 0204001030 & 0000203014 & 0203010004 & 88.6225 \\
\hline 0204001030 & 0023010040 & 3010040020 & 68.4233 \\
\hline 0204001030 & 0040200301 & 0040200301 & 107.8277 \\
\hline 0401032000 & 0003010402 & 0401032000 & 115.3927 \\
\hline 0401032000 & 0020300401 & 0400020301 & 101.6013 \\
\hline 0401032000 & 0023010040 & 0023010040 & 56.9143 \\
\hline 0402001030 & 0000403012 & 0403010002 & 115.3927 \\
\hline 0402001030 & 0020043010 & 2001030400 & 56.9143 \\
\hline 0402001030 & 0020400301 & 0020400301 & 101.6013 \\
\hline
\end{tabular}


Local network calculations around isomer 6:L: Single point DFT calculations at B3LYP-D3BJ/Def2-TZVPPD/CPCM (chloroform)

Vertex list:

\begin{tabular}{lrl} 
isomer & \multicolumn{2}{c}{ energy } \\
\hline----- & & \\
0000013402 & & 72.3739 \\
0000023401 & & 69.3774 \\
0000100342 & & 68.2481 \\
0000200341 & & 62.3759 \\
0000340102 & & 68.2481 \\
0000340201 & 62.3759 \\
0003400012 & & 72.3739 \\
0003400021 & 69.3774 \\
0010020340 & 4.5784 \\
0010030204 & 32.5790 \\
0010200034 & 32.5790 \\
0010340020 & 4.5784 \\
0020030104 & 44.2888 \\
0020100034 & 44.2888 \\
0102004300 & 43.2559 \\
0104302000 & 43.2559 \\
0201004300 & 43.6978 \\
0204301000 & 43.6978 \\
0431002000 & 62.1132 \\
0432001000 & 62.1132
\end{tabular}

Edge list:

\begin{tabular}{lrllll} 
isomer1 & isomer2 & \multicolumn{3}{c}{ trans.state TS energy } & \\
-0010020340 & 0104302000 & 0020340010 & 70.8518 \\
0010020340 & 0201004300 & 0340010020 & 76.3665 \\
0010020340 & 0432001000 & 0010020340 & 76.2222 \\
0010340020 & 0102004300 & 0340020010 & 70.8518 \\
0010340020 & 0204301000 & 0010340020 & 76.3665 \\
0010340020 & 0431002000 & 0020010340 & 76.2222 \\
0102004300 & 0000100342 & 0100340002 & 130.2448 \\
0102004300 & 0010340020 & 0340020010 & 70.8518 \\
0102004300 & 0020100034 & 0020100034 & 88.7353 \\
0104302000 & 0000340102 & 0104302000 & 130.2448 \\
0104302000 & 0010020340 & 0020340010 & 70.8518 \\
0104302000 & 0020030104 & 0100020034 & 88.7353 \\
0201004300 & 0000200341 & 0200340001 & 134.7071 \\
0201004300 & 0010020340 & 0340010020 & 76.3665 \\
0201004300 & 0010200034 & 0010200034 & 96.7404 \\
0204301000 & 0000340201 & 0204301000 & 134.7071
\end{tabular}




$\begin{array}{llll}0204301000 & 0010030204 & 0200010034 & 96.7404 \\ 0204301000 & 0010340020 & 0010340020 & 76.3665 \\ 0431002000 & 0000013402 & 0013400002 & 126.1078 \\ 0431002000 & 0003400021 & 2000431000 & 112.2086 \\ 0431002000 & 0010340020 & 0020010340 & 76.2222 \\ 0432001000 & 0000023401 & 0023400001 & 112.2086 \\ 0432001000 & 0003400012 & 1000432000 & 126.1078 \\ 0432001000 & 0010020340 & 0010020340 & 76.2222\end{array}$

